\title{
USE OF VIBRATION DATA FOR STRUCTURAL HEALTH MONITORING OF BRIDGES
}

\author{
by \\ Nicolás A. Londoño
A thesis submitted to
the Faculty of Graduate Studies and Research
in partial fulfillment of the requirements
for the degree of
Doctor of Philosophy

Department of Civil and Environmental Engineering

Carleton University, Ottawa

September, 2006

The Doctoral Program in Civil and Environmental Engineering is a joint program with the University of Ottawa, Administered by the Ottawa-Carleton Institute for Civil Engineering

(c) Copyright 2006, Nicolás A. Londoño 


$\begin{array}{ll}\begin{array}{l}\text { Library and } \\ \text { Archives Canada }\end{array} & \begin{array}{l}\text { Bibliothèque et } \\ \text { Archives Canada }\end{array} \\ \begin{array}{l}\text { Published Heritage } \\ \text { Branch }\end{array} & \begin{array}{l}\text { Direction du } \\ \text { Patrimoine de l'édition }\end{array} \\ \begin{array}{l}\text { 395 Wellington Street } \\ \text { Ottawa ON K1A ON4 }\end{array} & \begin{array}{l}\text { 395, rue Wellington } \\ \text { Ottawa ON K1A ON4 } \\ \text { Canada }\end{array}\end{array}$

Your file Votre référence ISBN: 978-0-494-18224-6 Our file Notre référence ISBN: 978-0-494-18224-6

NOTICE:

The author has granted a nonexclusive license allowing Library and Archives Canada to reproduce, publish, archive, preserve, conserve, communicate to the public by telecommunication or on the Internet, loan, distribute and sell theses worldwide, for commercial or noncommercial purposes, in microform, paper, electronic and/or any other formats.

The author retains copyright ownership and moral rights in this thesis. Neither the thesis nor substantial extracts from it may be printed or otherwise reproduced without the author's permission.
AVIS:

L'auteur a accordé une licence non exclusive permettant à la Bibliothèque et Archives Canada de reproduire, publier, archiver, sauvegarder, conserver, transmettre au public par télécommunication ou par l'Internet, prêter, distribuer et vendre des thèses partout dans le monde, à des fins commerciales ou autres, sur support microforme, papier, électronique et/ou autres formats.

L'auteur conserve la propriété du droit d'auteur et des droits moraux qui protège cette thèse. $\mathrm{Ni}$ la thèse ni des extraits substantiels de celle-ci ne doivent être imprimés ou autrement reproduits sans son autorisation.
In compliance with the Canadian

Privacy Act some supporting forms may have been removed from this thesis.

While these forms may be included in the document page count, their removal does not represent any loss of content from the thesis.
Conformément à la loi canadienne sur la protection de la vie privée, quelques formulaires secondaires ont été enlevés de cette thèse.

Bien que ces formulaires aient inclus dans la pagination, il n'y aura aucun contenu manquant.

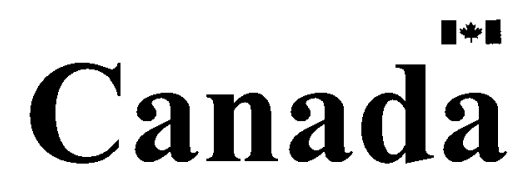




\section{Abstract}

Accurate and timely assessment of maintenance and repair requirements of bridges is important for ensuring public safety and for efficient allocation of limited resources by road transportation authorities. Periodic visual inspections have so far been the typical approach in assessing the condition of bridge structures. But the visual inspection approach is subjective as the results can vary from operator to operator, and it may fail to reveal hidden deficiencies that may affect the integrity of the inspected structures. Structural health monitoring (SHM) based on some form of structural response measurement has emerged as an alternative means to provide a quantitative and more accurate structural condition assessment. The techniques of monitoring vibration responses to ambient loads, such as wind and traffic are ideally suited for this purpose since theoretically any changes in stiffness due to damage or deterioration of the monitored structure are reflected in its vibration patterns. Furthermore, the monitoring of vibration responses of bridges to operational loads can be implemented cost effectively in relation to its potential benefits and does not lead to service disruptions.

This thesis presents and elaborates upon the different aspects of data manipulations involved in vibration-based SHM, including data processing, system identification, damage detection, and the development of computer tools to facilitate data analysis, visualization and interpretation of results. One of the main challenges in SHM using vibration data in practical applications in the field is the variability in the data arising from uncertainties in the environmental and loading operational conditions, as well as errors in modelling, measurement and computation, all of which can obscure the effects of damage or deterioration of the monitored structure. The question of whether the conditions of large 
complex civil engineering structures in the field can be realistically assessed using vibration response data has not so far been satisfactorily answered. This thesis examines this issue in detail using the monitoring data from the Confederation Bridge, in Canada. The Confederation Bridge is a large complex bridge located in a severe marine environment. The monitoring project of the Confederation Bridge provides the unique data used in this work to realistically assess the implications of the actual field observed variability of monitoring data on the feasibility of using vibration response data for structural condition assessment. The results obtained through a finite element updating damage detection algorithm indicate that continuous monitoring using multiple independent datasets is necessary to offset the errors arising from variability in the data to make it feasible to extract meaningful and useful information regarding the existence, location and magnitude of changes in stiffness associated with damage or deterioration from the monitoring data. 


\section{Acknowledgements}

I wish to express my gratitude to the people and institutions who made this thesis possible. Above all, to the Department of Civil Engineering at Carleton University for the facilities it offers for research, in particular to the Faculty of Graduate Studies for the Scholarship and Teaching Assistantship which provided me with the necessary funding to pursue graduate studies and for awarding the Jag Mohan Graduate Student Fellowship for Civil and Environmental Engineering for 2004-2005 and the John Adjeleian Graduate Scholarship for 2005-2006.

I owe special thanks to my thesis supervisor, Professor David T. Lau, who gave me the opportunity of participating in the Confederation Bridge Research Project as a Research Assistant. I profited from doctor Lau's careful and thoughtful observations, and from his constant support and advice at all stages of my work, both in Canada and abroad when attending various international conference presentations.

I would like to thank Mr. Donald McGinn and the staff of Strait Crossing Bridge Limited for their assistance during various trips to the Confederation Bridge, as well as John Egan and Alain Solari of Public Works and Government Services of Canada for their technical assistance, both with remote data collection and with in situ guidance at the mentioned bridge. I also want to acknowledge the help of my colleague Serge L. Desjardins, who in 2004 devoted himself fully to work with me on the development of the software platform for the processing and analysis of the monitoring data. I appreciated his family's hospitality at Moncton during one of my trips to the bridge. 
I would like to acknowledge the assistance by Professor Guido De Roeck from the Catholic University of Leuven, Belgium, whose detailed observations on the finite element updating method were instrumental for my research in that area.

My deepest gratitude goes to Marcela Dereix for her company and support while we lived in Ottawa; to my mother and relatives in Colombia, for their unconditional encouragement and support; and to my life-long friends Dario Jaramillo, Juan Camilo Sierra, Juan David Ramírez and Federico Carrillo for their generosity and for always being there.

Finally, I want to mention my friends in Ottawa, especially Ali and Julie, Stelios and Katerina, José and Silvia, Kate, Tuan, Ryan, Viet and Linh, Freddy and Claudia. They made my years in Canada a rewarding and unforgettable experience. 


\section{Contents}

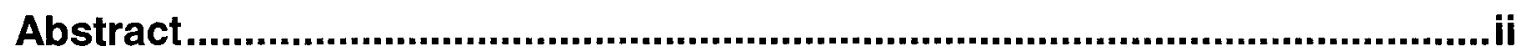

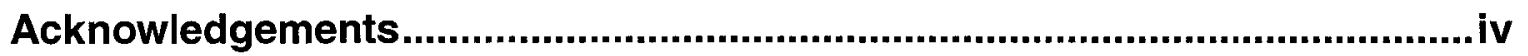

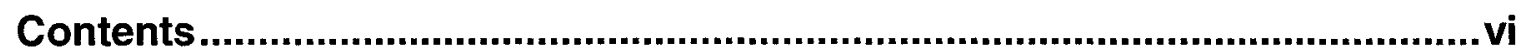

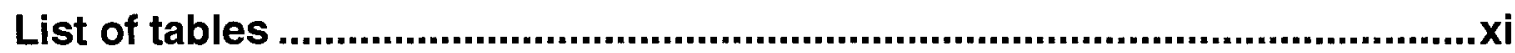

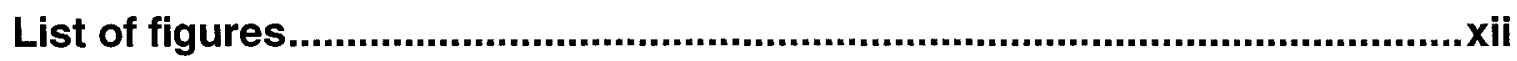

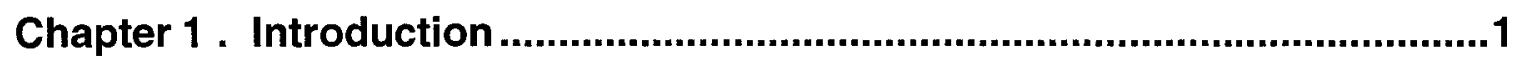

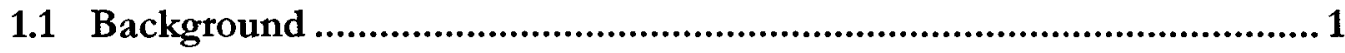

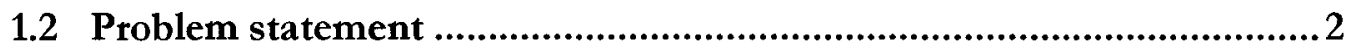

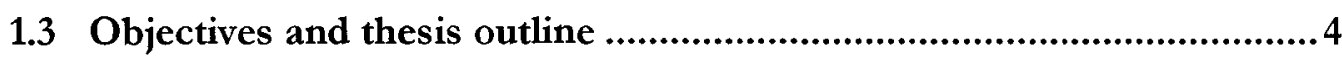

Chapter 2. Characteristics of dynamic monitoring data and observed behaviour of the Confederation Bridge................................................

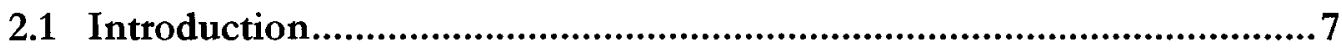

2.2 Continuous monitoring system .............................................................. 10

2.3 Monitoring data ..........................................................................................11

2.4 Typical vibration behaviour .................................................................... 12

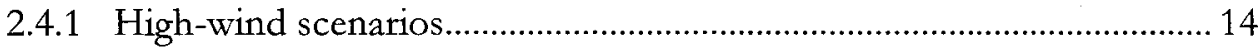

2.4.2 Traffic triggered scenarios................................................................... 14

2.4.3 Ambient scenatios ………………………………………………………... 15

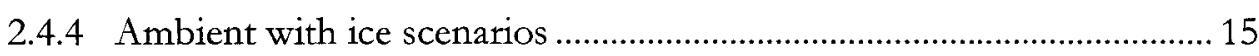

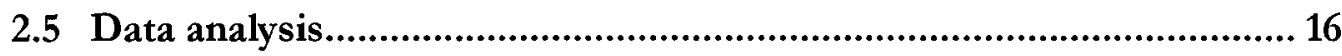

2.5.1 Data processing ……………………………………………………...... 16 


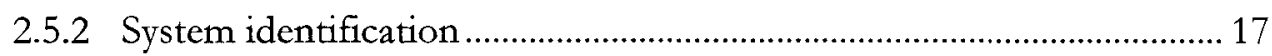

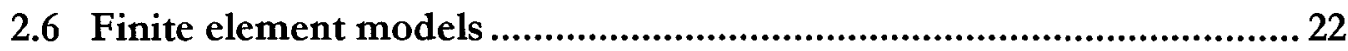

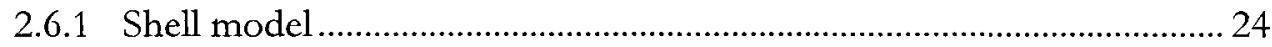

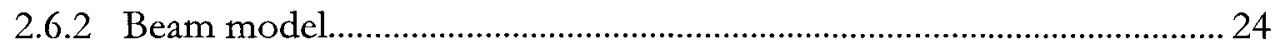

2.7 Analysis results from monitoring data ............................................ 25

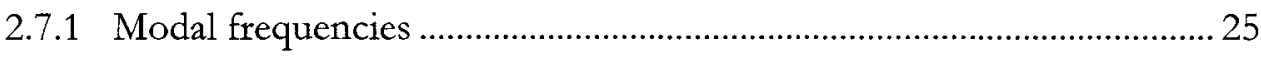

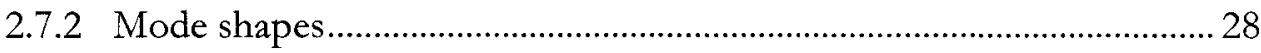

2.7.3 Modal damping ratios ........................................................................... 31

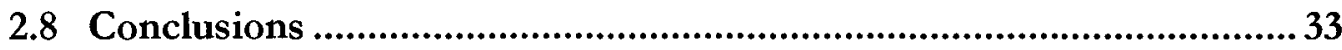

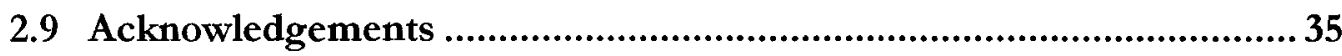

\section{Chapter 3 . Variability of dynamic properties from field data and} implications for health monitoring .................................................50

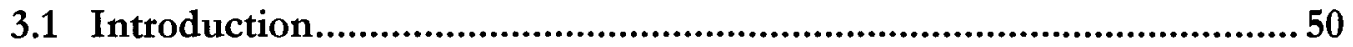

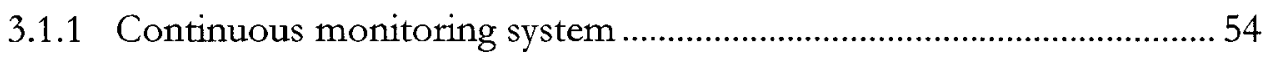

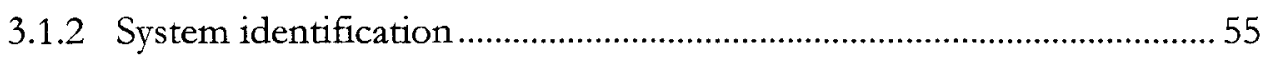

3.2 Potential sources of variability …...............................................5 56

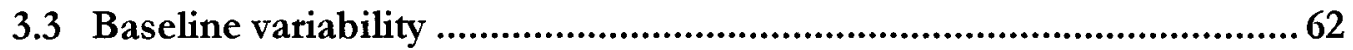

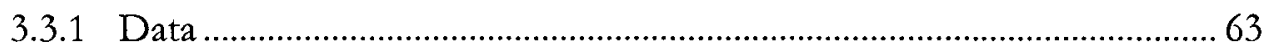

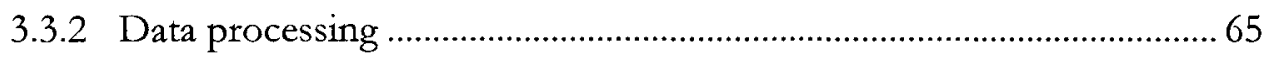

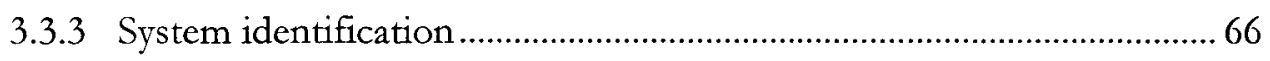

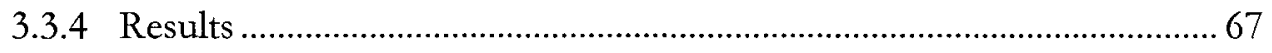

3.4 Variability under environmental \& loading fluctuations .....................69

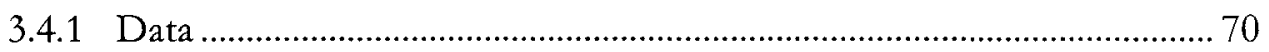

3.4.2 Data processing and system identification......................................... 71

vii 


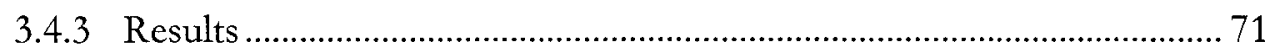

3.5 Variability of modal parameters vs. sensitivity to damage ................... 76

3.5.1 Results - pier stiffness degradation scenarios .....................................

3.5.2 Results - damage at continuous drop-in span joints............................. 79

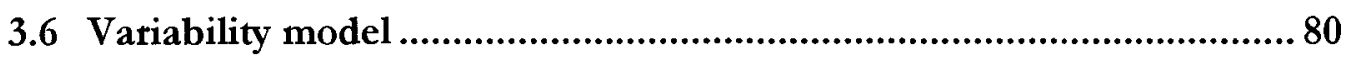

3.6.1 Amplitude dependent mode shape variability model........................... 80

3.6.2 Noise simulation for damage detection ............................................ 81

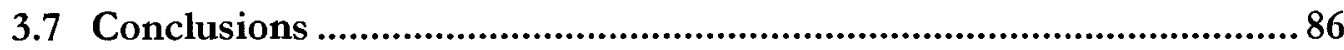

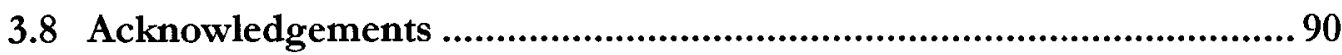

Chapter 4 . Application platform for processing, analysis and visualization

of Confederation Bridge monitoring data ....................................123

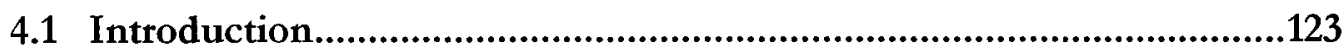

4.2 Confederation Bridge monitoring data ....................................127

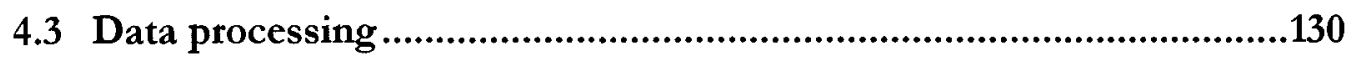

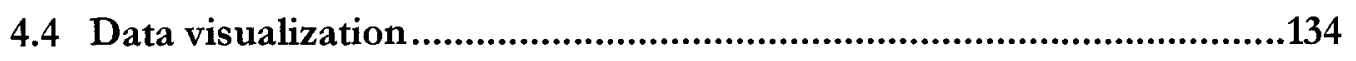

4.4.1 Real-time visualization ..................................................................... 135

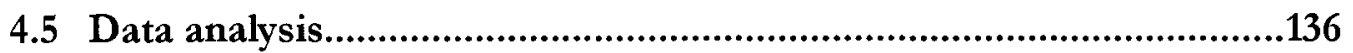

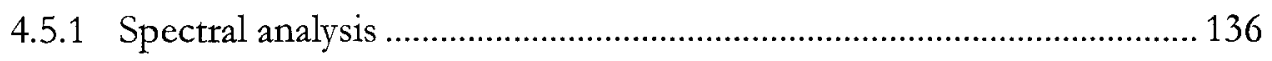

4.5.2 Stochastic subspace identification application tool .............................. 137

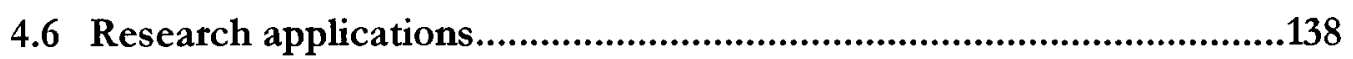

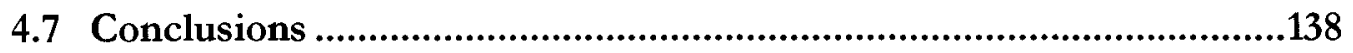

Chapter 5 . Damage detection techniques ..................................................145

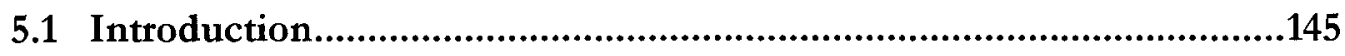

5.2 Damage detection via finite element updating...............................147 


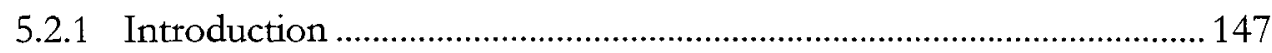

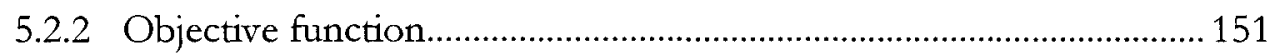

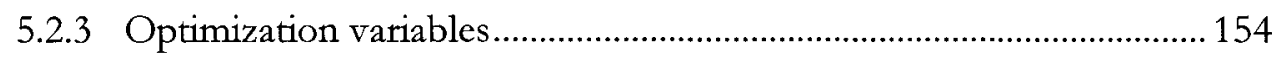

5.2.4 Optimization algorithm ...................................................................... 156

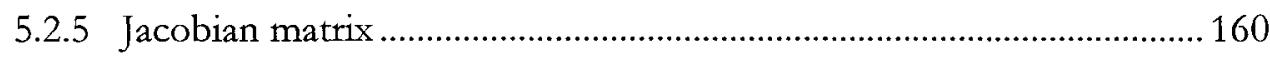

5.3 Modal strain energy damage index ..............................................162

5.4 Flexibility based damage detection ..............................................167

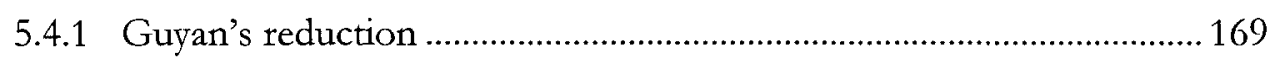

5.4.2 Modal strain energy vs. flexibility approach ..................................... 170

5.5 Application of flexibility-based damage detection ..........................171

5.5.1 Damage Locating Vectors (DLV) approach ..................................... 173

Chapter 6 . Application of finite element updating damage detection.......187

6.1 Damage detection of a simple bridge ............................................187

6.1.1 Structure and FE model ............................................................... 187

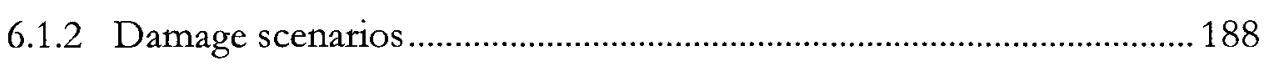

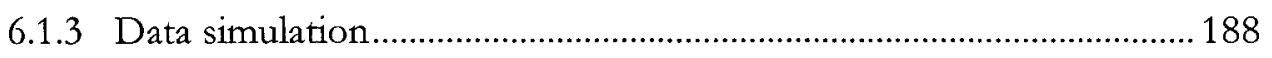

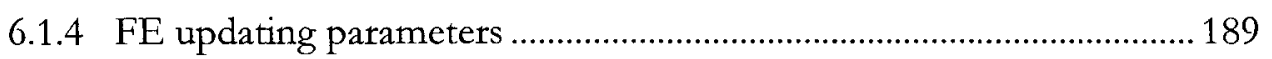

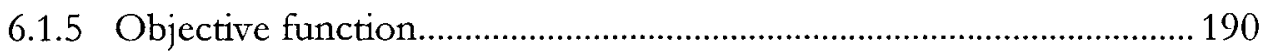

6.1.6 Damage detection results without variability .................................... 191

6.1.7 Damage detection results with variability ........................................... 193

6.2 Confederation Bridge damage detection ....................................195

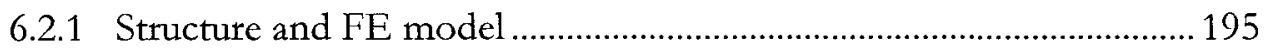

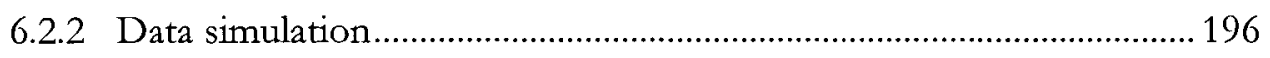

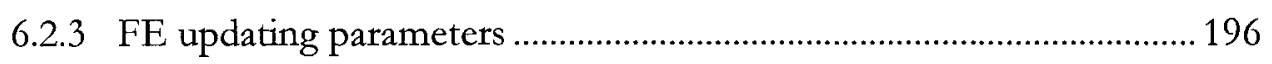

ix 
6.2.4 Damage scenarios ......................................................................... 198

6.2.5 Damage detection results without variability .................................... 201

6.2.6 Damage detection results with variability ….......................................204

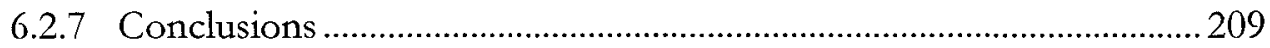

Chapter 7 . Conclusions and recommendations .......................................229

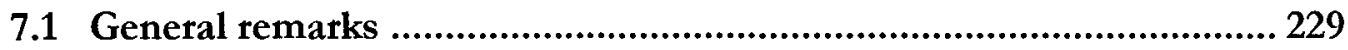

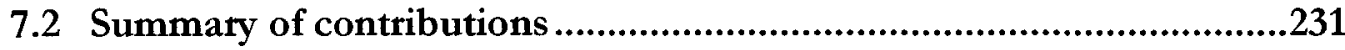

7.3 Recommendations for future research ......................................... 232

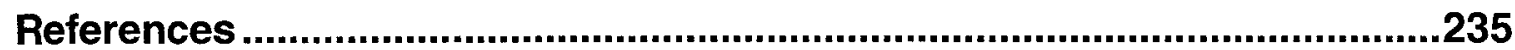




\section{List of tables}

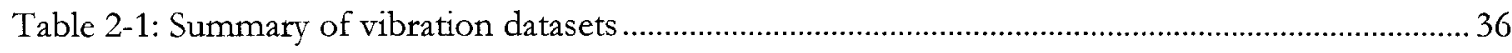

Table 2-2: Summary of modal frequencies from system identification of monitoring data and comparison to expected design values from finite element models

Table 2-3: Summary of modal damping ratios and Modal Assurance Criterion values for vibration

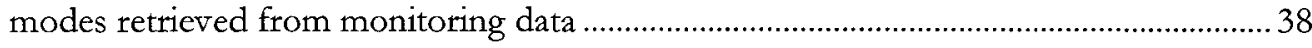

Table 3-1: Summary of baseline variability of extracted modal parameters............................................91

Table 3-2: Summary of variability of modal parameters over 6-month period.......................................92

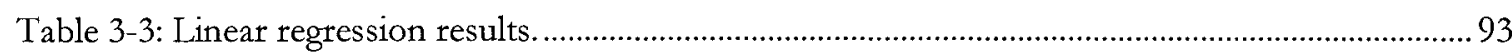

Table 3-4: Standard deviations of mode shape amplitudes. Values are given as percentage of the mean amplitude of the corresponding modal coefficient. Both baseline values and general values under random environmental fluctuations are given (the latter in parentheses).. 94

Table 3-5: Values of variability of mode shape amplitudes as predicted by rational polynomial least square fits

Table 5-1: Comparison of flexibility and modal strain energy damage detection techniques .178 


\section{List of figures}

Figure 2-1: (a) Dimensions and main components of typical structural module; (b) Locations of accelerometers in Confederation Bridge monitoring system.

Figure 2-2: Vertical and lateral acceleration time histories at monitoring location 9

Figure 2-3: Vertical and lateral frequency content at monitoring location 9.

Figure 2-4A: ANPSDs of bridge response signals. (a) Wind loading scenarios vertical direction (b) wind lateral (c) traffic vertical (d) traffic lateral

Figure 2-5: Typical stabilization diagram

Figure 2-6: (a) Shell element model of Confederation Bridge; (b) Beam element model schematic and model showing mesh.

Figure 2-7A: Vibration mode shapes shown in top view and side view. Labels indicate mean extracted frequency. Markers indicate sensor locations. Extracted modes are shown in full line, full markers; theoretical shown in dotted line, open markers. .46

Figure 2-8: Modal damping ratio versus modal frequency ................................................................... 49

Figure 3-1: Locations of accelerometers in Confederation Bridge monitoring system. ....................... 96

Figure 3-2: Environmental conditions of baseline variability datasets. ..................................................97

Figure 3-3: Vertical and lateral time histories at monitoring location 7 of baseline variability datasets.

Figure 3-4: Frequency content of bridge responses at location 7 of baseline variability datasets.........99

Figure 3-5: Typical data correlation estimates. 100

Figure 3-6: Vibration mode shapes. Full line: modes identified from the monitoring data; dotted line: modes from finite element model (not available for $1.62 \mathrm{~Hz}$ mode). .101

Figure 3-7: Baseline variability of extracted modal parameters. Eigenfrequencies and damping ratios are normalized by the mean values. Symbol "•" is for lower order SSI solutions; " $\triangle$ " is for higher order solutions. 102

$x i i$ 
Figure 3-8: General variability of extracted modal parameters. Eigenfrequencies and damping ratios are normalized by the mean values. Symbol " $\bullet$ " is for lower order SSI solutions; " $\triangle$ " is for higher order solutions 103

Figure 3-9: Baseline variability of identified mode shape coefficients under similar environmental and loading conditions, from analysis of 10 vibration datasets collected over a two-day period. 104

Figure 3-10: General variability of identified mode shape coefficients under operational conditions, from analysis of 22 ambient vibration datasets with fluctuating loading and environmental scenarios over six-month period. .105

Figure 3-11: Variability of identified mode shapes. Average normalized mode shapes are shown with error bars indicating \pm one standard deviation. For vertical vibration modes (all except $0.47 \mathrm{~Hz}$ mode), full-line indicates north side of girder, dotted line: south side of girder 106

Figure 3-12(a): Vertical and lateral acceleration time histories at monitoring location 7 (datasets 1 to 11). 107

Figure 3-13(a): Frequency content of bridge responses at monitoring location 7 (datasets 1 to 11)..109

Figure 3-14: Typical thermocouple layout 111

Figure 3-15: Wind speeds and average concrete temperatures at Confederation Bridge from August 2003 to mid January 2004. Values for the datasets of the variability study are indicated.

Figure 3-16: Modal frequencies vs. average temperature of the concrete of the bridge girder. The symbols are: "•" for lower order SSI solutions; " $\triangle$ " for higher order SSI solutions....113

Figure 3-17: Identified damping ratios of $1.63 \mathrm{~Hz}$ mode against vibration amplitude standard deviations of reference channel 7V1. .114

Figure 3-18: Locations of damage in simulated damage scenarios, shown in thicker lines. .115

xiii 
Figure 3-19: Sensitivity of modal frequencies to damage. (a) Pier deterioration scenarios, (b)

Scenarios of damage at joints of continuous drop-in span. Dotted line: average baseline variability of modal frequencies; dash-dotted line: average variability under loading and environmental fluctuations. 116

Figure 3-20: Sensitivity of mode shapes to damage scenarios of uniform pier deterioration with stiffness reductions of (a) $2 \%$, (b) $5 \%$ and (c) $10 \%$. Dotted line: average baseline variability MAC; dash-dotted line: average MAC under loading and environmental fluctuations

Figure 3-21: Sensitivity of mode shapes to damage at joints of continuous drop-in span with joint element inertia reductions of (a) $50 \%$ and (b) $75 \%$. Dotted line: average baseline variability MAC; dash-dotted line: average MAC under loading and environmental fluctuations.

Figure 3-22: Mode shape changes after simulated 10\% pier stiffness deterioration; (a) $5.57 \mathrm{~Hz}$ and (b) $5.70 \mathrm{~Hz}$. Full blue line: original mode shape; red dotted line: mode shape after damage.

Figure 3-23: Mode shape changes after a simulated 75\% stiffness reduction at the joints of a continuous drop-in span; (a) $4.81 \mathrm{~Hz}$ and (b) $4.91 \mathrm{~Hz}$. Full blue line: original mode shape; red dotted line: mode shape after damage. 120

Figure 3-24: Variation of standard deviation of mode shape amplitudes with mode shape amplitudes extracted from (a) Baseline datasets, (b) General datasets. 121

Figure 3-25: Schematic flow chart of a finite element updating damage detection process ................122

Figure 4-1: Schematic of Confederation Bridge dynamic data acquisition and transmission..............140

Figure 4-2: GUIs (a) data processing module; (b) file management module.........................................141

Figure 4-3: Graphical user interfaces (a) data display module; (b) animation module .........................142

Figure 4-4: Schematic of real-time data processing and animation ...................................................... 143

Figure 4-5: GUI for system identification data analysis with stabilization diagram .............................144

xiv 
Figure 5-1: Construction of piece-wise linear damage functions over finite element mesh. (a) Local damage shape function, (b) global damage shape function. .179

Figure 5-2: Flow chart of subroutines of implemented FE updating damage detection algorithm for use in damage detection simulation study.

Figure 5-3: Percentage change in flexibility (from Eq. (5-49)) for each of the 6 DOF directions $(\Delta x$, $\Delta y, \Delta z, \theta x, \theta y, \theta z)$, in corresponding order from top to bottom. Assuming all FE DOFs could be instrumented. Amplification factors: $[10,1,0.3,1,1,2.5]$; 181

Figure 5-4: Percentage change in flexibility (from Eq. (5-49)) for each of the 3 measured DOF

directions $(\Delta \mathrm{x}, \Delta \mathrm{y}, \Delta \mathrm{z})$, in corresponding order from top to bottom, assuming instrumented DOFs are only those shown in 182

Figure 5-5: Simple valley bridge; (a) Element and joint numbers of finite element model; (b) member cross sections

Figure 5-6: "Measured" DOFs for the valley bridge. 184

Figure 5-7: Average normalized element stress field obtained via DLV approach for damage scenario 1 of the "valley" bridge example. Regions of near-zero stress are indicative of damage. An amplification factor of 2 is used for the plot. 185

Figure 5-8: Average normalized element stress field obtained via DLV approach for damage scenario 1 of the "valley" bridge example using exact flexibility matrix. An amplification factor of 0.3 is used for the plot 186

Figure 6-1: Damage elements (in italics) and piece-wise linear damage shape functions....................211

Figure 6-2: modes 1 to 8 used for damage detection 212

Figure 6-3: Distribution of element stiffness correction factors $c_{e}$ identified from 5 simulations of the FE updating algorithm with different starting points using (a) FE modes 1 to 8 as "measured modes"; (b) only FE modes 2, 3, 5, 8 as "measured modes". A scale of $1 \mathrm{~m}$ $=0.1$ correction factor is used. Positive $c_{\mathrm{e}}$ 's plotted up $(\uparrow)$ for girders, to the right $(\rightarrow)$ for piers. Symbols simulations 1 to $5:$ : $\bullet "$, , “-o", “-- ", “_- ", “- ” 
Figure 6-4: Distribution of element stiffness correction factors " $\mathrm{c}_{e}$ " identified from 5 simulations of the FE updating algorithm with different starting points using (a)only FE modes 1 to 8 as "measured modes"; (b) only modes: $2,3,5,8$. A scale of $1 \mathrm{~m}=0.1$ correction factor is used. Symbols for simulations 1 to 5: "“-•", "-o", “-- ", “-- ", “"- "

Figure 6-5: Element stiffness correction factors " $\mathrm{c}_{\mathrm{e}}$ " identified for damage scenario 3 from 5 simulations of the FE updating algorithm with different starting points, using modes 1 to 8 as "measured modes". (a) Results with regular damage mesh; actual damage pattern shown in dotted line; linear least squares fit to the actual damage shown in thicker dashed line; (b) results with refined mesh. Scale $1 \mathrm{~m}=10 \%$ correction factor. Symbols

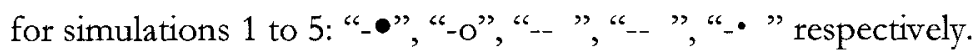
.215

Figure 6-6: Refined damage element mesh and damage functions for damage scenario 3. 216

Figure 6-7: Distribution of element stiffness correction factors $c_{e}$ identified from 5 simulations of the FE updating algorithm using FE modes 1 to 8 as "measured modes" with different starting points and (a) fixed random noise in the measurements Jacobian matrix condition number at the solution is 123); (b) different random noise in the measurements (condition number at solutions varies between 114 and 123). (c) Average of "(b)". A scale of $1 \mathrm{~m}=0.1$ correction factor is used. Symbols for simulations 1 to 5 : “-•", “-о", “-- ", “-- ", “_- ", .217

Figure 6-8: (a) Dimensions and main components of typical structural module; (b) ...........................218

Figure 6-9: "Sensor" locations used in the damage detection simulations. .219

Figure 6-10: Modes of Confederation Bridge used as "measured" modes for damage detection.....220

Figure 6-11: Damage element mesh and local damage functions for Confederation Bridge .221

Figure 6-12: Bridge pier cross section showing location of simulated damage considered in damage scenario 1 , indicated by dotted line. 222

Figure 6-13: Bridge profile showing the location of simulated damage in girder joint considered in damage scenario 2 , indicated by dotted line. .223 
Figure 6-14: Identified pattern of element stiffness correction factors $c_{e}$ identified from 5 simulations of the FE updating algorithm with different starting points for damage scenario 1; (a) without noise in the measured modal properties; (b) and (c) with noise; (d) with different noise realizations; (e) average of “(d)". Symbols for simulations 1 to 5 : “-•", “o", “-- ", “-- ", “-. ". Scale: $6 \mathrm{~m}=0.1$ correction factor. Positive $c_{\mathrm{e}}$ plotted up $(\uparrow)$ for girders, to the right $(\rightarrow)$ for piers. The actual damage pattern is shown in dotted line.224

Figure 6-15: Identified pattern of element stiffness correction factors $c_{e}$ identified from 5 simulations of the FE updating algorithm with different starting points for damage scenario 2; (a) without noise in the measured modal properties; (b) actual damage pattern (dotted), best fit of damage function to the actual damage (dashed); (c) with noise; (d) with different noise realizations; (e) average of “(d)". Scale: $6 \mathrm{~m}=0.1$ correction factor. Symbols for simulations 1 to 5 : “-•", "-o", “-- ", “-- ", “-• " .225

Figure 6-16: Refined mesh for damage scenario 2, based on preliminary damage detection. .226

Figure 6-17: Identified pattern of element stiffness correction factors $c_{e}$ identified from 5 simulations of the FE updating algorithm with different starting points for damage scenario 2 using refined mesh; (a) without noise in the measured modal properties; (b) with different noise realizations; (c) average of the results of "(b)". Scale: $6 \mathrm{~m}=0.1$ correction factor.

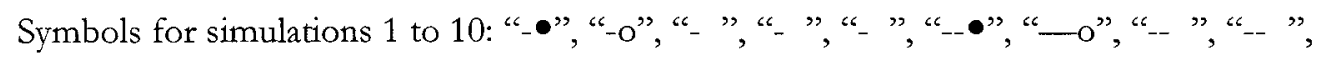
“.”. .227

Figure 6-18: Identified pattern of element stiffness correction factors $c_{e}$ identified from 5 simulations of the FE updating algorithm with different starting points for damage scenario 3. (a) Damage of all piers, without noise; (b) damage of piers within instrumented segment, without noise; (c) with different noise realizations; (d) average of "(c)". Scale: $6 \mathrm{~m}=0.1$

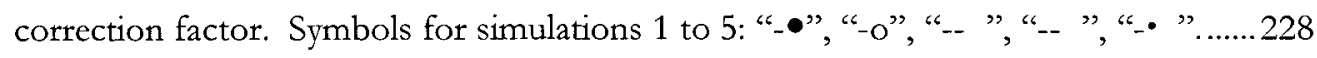




\section{Chapter 1. Introduction}

\subsection{Background}

This thesis pertains to the practical application of vibration based structural health monitoring (VBSHM) techniques in Civil Engineering structures. The objective of VBSHM of Civil Engineering structures is to assess the conditions of the structures (such as bridges, dams, towers, etc) using data and information obtained from vibration response measurements.

The assessment of structural condition to determine maintenance and repair needs of civil engineering structures, such as highway bridges, has traditionally relied upon periodic visual inspections (FHWA 1995, Yanev 2003), sometimes aided by local destructive or nondestructive techniques, including those based on x-rays, acoustics, ultrasound or magnetic fields, which can give detailed information about the local physical condition of the structural components and materials (Chang and Liu 2003). Despite advances in local non-destructive condition assessment methods and devices, the visual inspection-based approach has several important limitations such as inaccessibility to some parts of the structure, the need to know the damage location a-priori, making it difficult to detect hidden internal damage and the fact that the condition surveys can only be carried out at periodic intervals. Also, visual inspections do not generally provide a quantitative measure of how the local damage or distributed deterioration affects the global integrity and behaviour of the structure.

Consequently, in recent years increasing research efforts have been directed towards vibration based health monitoring (VBSHM) as a viable alternative with the advantage that vibration responses of a monitored structure can provide a "signature" of its global structural condition. The basic premise of VBSHM is that for a "healthy" structure, the physical mass 
and stiffness characteristics remain constant. Therefore, the vibration properties of the structure, such as mode shapes and frequencies, should theoretically remain invariant since these are directly related to mass and stiffness properties of the structure. Hence, any changes in the vibration properties can be attributed to structural damage or deterioration. Through the solution of an inverse problem, it should be possible to determine the location, extent and severity of the damage.

The development of VBSHM technology specifically for Civil Engineering applications occurs at an opportune time to exploit the many advances and potentials of information technology (IT). Because of the fast becoming common availability and everexpanding capacities of high-speed broadband cyber infrastructure in Canada and many parts of the world, it is now possible to transmit and process continuous vibration response data collected from remotely monitored structures located far away from the monitoring station or distributed over a wide area. From the perspective of infrastructure asset management, VBSHM techniques coupled with enabling IT technologies have the potential to allow the monitoring of large infrastructure networks on an online basis. Critical components of infrastructure networks, such as bridges, can be diagnosed automatically and timely information regarding the condition of the structures can be provided to operators in nearreal time. This would not only be very useful for rapid assessment of lifelines and other critical facilities providing support for decision making in emergency response following the occurrence of natural or man-made disasters such as earthquakes, hurricanes and collisions, but also for planning of maintenance.

\subsection{Problem statement}

Practical applications of vibration-based structural health monitoring often encounter difficulties in the field. The accuracy and reliability of many damage detection algorithms are 
seriously affected by their low sensitivity to damage, incomplete knowledge of the measured vibration characteristics and masking of the damage by noises in the data (Humar et al. 2006). While each of these issues represents a significant challenge on its own, the one with the most significant impact is the influence of noises and uncertainties in the monitoring data. The findings of recent extensive literature reviews on the subject of structural health monitoring corroborate that the variability of the data under operational conditions is a research priority (Doebling et al. 1996, Sohn et al. 2003). The sources of uncertainty in the monitored structural properties can be classified into two groups. First, there are "numerical" uncertainties such as those attributable to measurement errors, deviations of the data from modeling assumptions and computational errors caused by epistemic uncertainties of analysis methods and algorithms. On the other hand, there are "physical" uncertainties, which differ from the "numerical" in that they can induce changes in the structural dynamic system. These include the changes in the environmental and loading conditions, which tend to be random and widely fluctuating variables in the operational settings of most civil structures in the field.

This thesis addresses this problem by using field monitoring data from the Confederation Bridge monitoring project. This comprehensive monitoring project provides a unique opportunity to understand the behaviour and uncertainties of real-world field monitoring data from a large and complex structure located in a severe operating environment that regularly experiences a wide range of operational and loading conditions throughout the year, and as a result of this understanding to make significant advances towards the practical use of the monitoring data for structural health monitoring of civil structures. 


\subsection{Objectives and thesis outline}

This thesis is organized into eight chapters. The first part of the work presented in Chapter 2 is a study on the vibration characteristics of the Confederation Bridge extracted from field vibration monitoring data. The study provides essential information for further studies related to the vibration based health monitoring of the structure presented in subsequent chapters. The aims of Chapter 2 are as follows:

(i) To identify the global modal vibration properties (frequencies, mode shapes, and damping ratios) of the Confederation Bridge from the collected monitoring data.

(ii) To identify patterns in the dynamic behaviour such as determining which vibration modes are excited under each of the different loading scenarios (traffic, wind, ice floes).

(iii) To verify that the actual response behaviour of the bridge corresponds to the expected design behaviour. Important design assumptions regarding the dynamic behaviour, such as the material properties and damping ratios are verified.

The second part of the study, presented in Chapter 3, focuses on the issue of variability of the dynamic properties extracted from the vibration monitoring data. The variability, which results from a combination of different uncertainties in the extracted dynamic properties, represents a major challenge towards practical implementation of VBSHM in the field. The objective of this part of the study is to determine whether damage can be detected, located and quantified reliably from changes in the extracted dynamic properties if the extracted properties exhibit variability that may be comparable or even larger in magnitude than the changes arising from damage. In this research, the potential sources of uncertainty are first identified and the variability in the dynamic properties extracted from the long-term monitoring data of the Confederation Bridge, as related to each 
of the main sources of uncertainty, is characterized and quantified in detail. Then, the sensitivity of the dynamic properties of the bridge to simulated damage scenarios is determined using finite element models of the bridge. Finally, the variability and sensitivity results are compared to evaluate whether it is likely or not that the changes in the dynamic properties arising from damage could be masked by the observed variability. In addition, Chapter 3 also presents the development of a model for the simulation of noise in the identified structural properties in accordance with the observed behaviour of the data. The model is used later in the damage detection simulation studies discussed in Chapter 6.

Chapter 4 presents the global concepts as well as some of the relevant details of the software platform jointly developed with Desjardins (Desjardins 2004, Desjardins et al. 2006) for the management and analysis of the monitoring data. The application consists of several integrated modules to carry out different tasks involved in the manipulations of the monitoring data. The integrated application platform has a user friendly interface which facilitates the extraction and interpretation of meaningful engineering information from the data. The main aspects of the architecture of the various application modules, which include data management, data processing, data plotting and spectral analysis, system identification analysis, and response visualization interfaces, are discussed in details.

Chapter 5 presents the theoretical basis for the study of the implications of the variability findings reported in earlier chapters on the feasibility of conducting structural condition assessment of civil engineering structures using field measured vibration response data. Three different damage detection algorithms selected from the literature based on previous performance evaluation results are presented followed by a discussion on the advantages and drawbacks of each technique. The first technique selected is based on changes in the modal strain energies, the second is based on finite-element updating - in turn 
based directly upon mode shape and frequency changes -, and the third is based upon changes in the flexibility matrix of the structure, which can be estimated from the data. From the results of preliminary evaluations, it is found that there is limitation in the flexibility based damaged detection technique as a practical structural health monitoring tool. The different physical concepts and the various assumptions behind each of the damage detection techniques are discussed.

In Chapter 6, the performance and practical potential of the finite element updating damage detection strategy as a structural condition assessment tool is evaluated through realistic damage detection simulations that incorporate the appropriate measured levels of noise and uncertainties in the monitoring data as obtained from the variability study of this research. The implementation of the finite element updating damage detection method is first tested and verified by using a simple two-dimensional bridge structure before its application to damage detection investigations under different damage scenarios on the more complex model of the Confederation Bridge. The results using uncertain measured data and the implications of the research findings on the prospects of SHM technology in the field are examined in detail.

Finally, Chapter 7 presents the general conclusions of the thesis. 


\section{Chapter 2. Characteristics of dynamic monitoring data and observed behaviour of the Confederation Bridge}

\subsection{Introduction}

A permanent monitoring network installed on the Confederation Bridge, in Canada, captures the vibration responses of the structure resulting from ambient excitations such as wind, traffic, ice and earthquake loads. In the present study, monitoring datasets collected under different loading scenarios have been analyzed to identify the typical response behaviour of the structure, understand some of the essential characteristics of the monitoring data, and verify the expected dynamic behaviour of the bridge. The distinctly different frequency content characteristics of the bridge responses under different loading scenarios are identified, as well as the bridge's modal vibration properties, which are extracted from the different monitoring datasets using an output-only stochastic subspace technique. The variability observed in the modal vibration properties extracted from the different monitoring datasets is discussed. In general, the mean extracted properties are found to have good agreement with the design values. After calibration of the computer models using updated field measured material properties, the identified modal frequencies differ only by $3 \%$ to $4 \%$ on average from the expected values. The extracted mode shapes agree well with those determined from computer models. The damping ratios of the structure are also presented and compared against values assumed at the design stage. Understanding the behaviour of the dynamic responses and the variability of the modal properties extracted from field monitoring data is essential for vibration based structural assessment and health monitoring in the future. 
The Confederation Bridge is a prestressed concrete bridge crossing the Northumberland Strait in Eastern Canada. It provides the only fixed link between the provinces of New Brunswick, in mainland Canada, and Prince Edward Island. With a length of $12.9 \mathrm{~km}$, the Confederation Bridge is the world's longest continuous multi-span bridge operating in salt water (Cheung et al. 1997). To cover the long shore-to-shore distance the bridge is divided into 21 approach spans, two transition spans of $165 \mathrm{~m}$ each and 43 main spans of $250 \mathrm{~m}$ each at a typical height of $40 \mathrm{~m}$ above the mean sea level. The main-span portion of the bridge is comprised of 22 repetitive structural frame modules of $500 \mathrm{~m}$ length each. Each module is composed of a $440 \mathrm{~m}$ portal frame, made up of a $250 \mathrm{~m}$ centre span and two $95 \mathrm{~m}$ overhangs, one on each side of the centre span, plus a $60 \mathrm{~m}$ simply supported drop-in expansion span, as shown in Figure 2-1(a). The portal frame is constructed of four types of prefabricated component units, pier base, pier shaft, main girder and fixed drop-in span girder. The connections between the different units of the portal frame are made continuous by post tensioned pre-stressing and grouting. The bridge girders are single cell trapezoidal box girders with section depth varying from $14 \mathrm{~m}$ above the piers to $4.5 \mathrm{~m}$ at mid-span of the drop-in girder, and a cross-section width varying from $5.0 \mathrm{~m}$ at the bottom to $7.0 \mathrm{~m}$ at the top, with $2.5 \mathrm{~m}$ deck overhangs.

Due to the importance of this structure its design specifications and requirements are more stringent than those of typical highway bridges and are not covered in any bridge design standards and codes around the world. The main performance requirements of the project are the design service life of 100 years, which is twice that of ordinary structures, and a structural safety level quantified by the reliability index of $\beta=4.0$ for load effects with multiple load paths, and $\beta=4.25$ for load effects of single load path, as compared to the values for ordinary bridge structures of $\beta=3.0$ to $\beta=3.5$, respectively. To satisfy these 
stringent design specifications under the harsh environmental conditions of the Northumberland Strait, the designers of the Confederation Bridge had to overcome numerous challenges, including lack of data and information on the various design load effects of ice impact, traffic, wind and earthquakes. As a result, load combinations and resistance factors had to be specially developed for the project (MacGregor et al. 1997). With the completion of the Bridge and the experiences of its operation since 1997, there is the opportunity to assess and verify the behaviour and performance of this structure using the bridge response monitoring data collected since its opening to advance the knowledge base and practices of bridge engineering.

This chapter presents the extracted dynamic properties of the Confederation Bridge determined by output-only system identification of vibration monitoring data. The purpose of the study is not to establish a baseline reference for damage detection of the facility but rather to improve our understanding of the typical vibration behaviour of this important bridge and other similar large scale civil engineering structures in the field. The information and findings obtained will be useful for conducting detailed baseline studies and health monitoring of the facility in the future.

As opposed to traditional input-output modal testing of mechanical engineering, where structural responses to controlled excitation forces are measured, for critical lifeline civil structures such as the Confederation Bridge service interruptions for testing are not desirable plus it also tends to be too costly or impractical to use artificial sources of excitation due to the size and mass of the structures. Therefore, special system identification techniques relying only on measured response signals to ambient loadings - without knowledge of the forces - must be used because the natural excitation sources are not easily measurable or they lack detailed information. Use of these techniques allows the verification 
of design assumptions and performance of the structure through comparisons of the identified dynamic properties against expected design values predicted by finite element models based on construction specifications.

In this study, twenty-one vibration datasets under the different excitation scenarios typically encountered by the bridge have been analyzed. The system identification results of modal vibration frequencies, damping ratios and mode-shapes are presented and discussed. The dynamic properties and behaviour of the Confederation Bridge are investigated in a frequency range that essentially encompasses the global vibration behaviour of the structure under the range of dynamic loading scenarios typically encountered by the structure. These scenarios include the load effects of wind, ice floes and traffic.

\subsection{Continuous monitoring system}

A comprehensive long-term monitoring system on the Confederation Bridge has been in operation since the bridge opening in 1997 to collect data and information about its behaviour and performance. The monitoring system measures and records both environmental and bridge response data related to ice forces, short and long-term deflections, thermal effects, corrosion and dynamic responses. The dynamic monitoring system is dedicated to the measurement of the vibration responses of the bridge caused by significant sources of dynamic excitations, including wind, heavy traffic, ice loads and earthquakes. The vibration instrumentation comprises a total of 76 accelerometers distributed around a typical structural frame module. In this study, the recorded signals from 50 of these accelerometers are used, which are shown in Figure 2-1(b). Vibration responses of the bridge girders are measured in the vertical and lateral horizontal (herein forth referred simply as "lateral") directions, as shown in Figure 2-1(b). This setup facilitates the recovery of vertical bending, lateral bending and torsional vibration modes of the bridge superstructure. The response 
behaviour observed in the instrumented segment of the bridge is considered representative of the behaviour of the main-spans portion of the structure.

The vibration sensors used in the monitoring system include both piezo-electric accelerometers and servo accelerometers. The measured analog accelerometer signals are conditioned and filtered for anti-aliasing by a Frequency Devices 8-pole $50 \mathrm{~Hz}$ low-pass Bessel filter. Signals are then sampled using a strict sample and hold analog to digital conversion by a network of high-speed Campbell Scientific data loggers before being sent to on-site computers and transmitted back to Carleton University in Ottawa for data archival and research. Typical data sampling rates vary between $100 \mathrm{~Hz}$ and $167 \mathrm{~Hz}$. Dedicated communication lines between the different data loggers ensure simultaneous triggering and recording. The data loggers operate in continuous buffered data collection mode, which upon triggering by detection of specific dynamic events, such as heavy traffic signals or high winds, or simply upon user request, store time history response data in hard disk for detailed analysis and research. Otherwise, only statistic information determined from the time history data, such as mean, maximum, minimum and standard deviation, are stored.

The structural monitoring framework encompasses not only the monitoring instrumentation and data collection systems at the bridge site, but extends to also include the computer infrastructure for the distribution, processing and utilization of the monitoring data. Details of the monitoring system setup have been described in (Cheung et al. 1997, Montreuil et al. 1998), while the development of the associated computer tools are discussed by Desjardins et al. (2006).

\section{$2.3 \quad$ Monitoring data}

Twenty-one datasets of vibration responses of the Confederation Bridge under different dynamic loading scenarios collected by the continuous monitoring system have 
been analyzed and the results are presented in this study. Each dataset comprises the simultaneous acceleration time-history records of 90 -second duration from a total of 50 accelerometers, which are permanently installed at monitoring locations 1 through 18 between Piers 31 and 32. The datasets have been selected from the monitoring database according to strict criteria to ensure that they are representative of each of the loading scenario categories considered, i.e. "ambient", "high wind", "traffic", and "ambient with ice". At least four datasets are considered for each type of loading scenario. The datasets were collected from November 2000 to May 2002. It is assumed that the structural condition of the bridge does not significantly change during this period to allow for the comparison between the different datasets.

"Traffic" datasets are triggered by heavy traffic under low wind and no-ice conditions with transverse wind speeds lower than $20 \mathrm{~km} / \mathrm{h}$. An exception to this rule is allowed for one of the traffic datasets because it was collected under a controlled heavy truck traffic test (2000-12-14 00:13, transverse wind speed $=28 \mathrm{~km} / \mathrm{h})$. "Ambient" scenarios involve typical wind and traffic loading combinations with transverse wind speeds higher than $20 \mathrm{~km} / \mathrm{h}$. "High-wind" scenarios involve average transverse wind speeds in excess of $50 \mathrm{~km} / \mathrm{h}$ with little or no traffic. Criteria for the selection of the "ambient with ice" scenarios are essentially the same as the "ambient" counterparts except there is presence of significant moving ice floes. The ice thickness data in the Northumberland Strait at the bridge site are obtained from the report by Hayden et al. (2003) based on Acoustic Doppler Current Profiler (ADCP) measurements. Dataset information is summarized in Table 2-1.

\subsection{Typical vibration behaviour}

Figure 2-2 shows typical time domain plots of bridge acceleration responses. Responses at mid-span of the portal frame, at sensor location 9, from selected monitoring 
datasets are shown. Figure 2-3 shows the corresponding frequency domain plots. The figures illustrate the typical distinctive characteristics of the data under the different loading scenarios. For example, as shown in Figure 2-3, the bridge responses under the November 2001 wind storm, during which the bridge was closed for traffic, are clearly dominated by low frequency vibration modes below $1.0 \mathrm{~Hz}$, while the vibration response caused by heavy traffic at this location is dominated by higher frequency modes, particularly those in the 2.5$3.5 \mathrm{~Hz}$ range in the vertical direction, and those in the $11-14 \mathrm{~Hz}$ in the lateral direction.

A single sensor location is not sufficient to characterize the typical frequency content of the overall bridge response because the sensor could coincide with or be close to modal nodes. Hence, the frequency content of the signals is further examined using average normalized power spectral densities (ANPSDs) of multiple sensors for each of the loading scenarios. For the computation of the ANPSD for a given type of loading scenario, the power spectral densities (PSDs) of signals from all sensors and all datasets corresponding to that scenario are computed and the results are averaged. Each PSD is normalized by its area to give equal weight to all response signals. The PSDs of individual responses are computed from the $90 \mathrm{~s}$ time histories $(\Delta \mathrm{t}=0.02 \mathrm{~s})$ via Welch's method using the following estimation parameters: 3000-point segments, Hamming windowing, 50\% segment overlap, and 4096point FFT. This results in a frequency resolution of the ANPSDs of $\Delta f=0.012 \mathrm{~Hz}$. The ANPSDs serve the purpose of preliminarily identifying which are the dominant vibration modes of the structure under each scenario, which is important information needed for the health monitoring of the structure in the future. ANPSDs computed separately for responses in vertical and lateral directions are presented in Figure 2-4. 


\subsubsection{High-wind scenarios}

As shown in Figure 2-4A, the frequency content of responses collected under highwind scenarios are characterized by having most of the response energy in the low frequency range, mostly below $1.0 \mathrm{~Hz}$. In the vertical direction, two closely-spaced modes, at frequencies of $0.68 \mathrm{~Hz}$ and $0.64 \mathrm{~Hz}$, are dominant in the responses, followed by the $0.95 \mathrm{~Hz}$ mode. In the lateral direction, the $0.48 \mathrm{~Hz}$ mode is dominant, followed by a mode at 0.33 Hz.

\subsubsection{Traffic triggered scenarios}

The frequency content of the responses under heavy traffic is quite different from the frequency content associated with the high wind scenarios. For the case of traffic loading, the vibration response energy in the vertical direction is concentrated mainly in the $2.5 \mathrm{~Hz}$ to $3.5 \mathrm{~Hz}$ range, but also to some extent in the $1 \mathrm{~Hz}$ to $2 \mathrm{~Hz}$ range with the most important traffic excited modes in the vertical direction located at $3.44 \mathrm{~Hz}, 3.29 \mathrm{~Hz}$, and $2.74 \mathrm{~Hz}$, in order of importance. Some other peaks are also important, such as the ones at $0.66 \mathrm{~Hz}$, and $1.8 \mathrm{~Hz}$ in the vertical direction. In the lateral direction, the peak around $3.3 \mathrm{~Hz}$ corresponds to the first torsional mode of the bridge. Although this mode involves mainly the purely torsional motion of the girder about its longitudinal axis, it also involves significant associated lateral deflections. In general, the frequencies of the bridge response under traffic correlate well with the findings of vehicle-structure interaction studies (Cantieni 1993). Another observation is the presence, in both the vertical and lateral directions, of some significant response energy in the $9.0 \mathrm{~Hz}$ to $17 \mathrm{~Hz}$ frequency range. These higher frequency response components result from vibration modes that involve local warping deformations 
of the box-girder cross-section, as reported in previous studies (Zhang 2001, Naumoski 2002). ${ }^{1}$

\subsubsection{Ambient scenarios}

The "ambient" scenarios represent typical operating conditions where the bridge is subjected to random combinations of wind and traffic loading. In the "ambient" scenarios the bridge responses essentially exhibit a combination of the characteristics of the winddriven and traffic-driven responses described above. The vibration energy in the vertical direction is now distributed between the low-frequency wind-excited modes and the midfrequency traffic excited modes up to around $5.2 \mathrm{~Hz}$. The peak responses in the vertical direction are observed at $0.67 \mathrm{~Hz}, 2.74 \mathrm{~Hz}$ and $3.29 \mathrm{~Hz}$ while the most important modes in the lateral direction are located at $0.49 \mathrm{~Hz}$ and $0.38 \mathrm{~Hz}$. The $3.3 \mathrm{~Hz}$ torsional-lateral mode is less prominent under the ambient scenario than under the traffic scenarios.

\subsubsection{Ambient with ice scenarios}

During the winter season, for approximately three months of the year, ice floes of the Northumberland Strait induce significant static and dynamic loading effects on the Confederation Bridge. The behaviour of ice interactions with the structure under extreme ice loading conditions has been the subject of past studies (Langohr and Ghali 1997, Brown 2000). In contrast to those studies, the interest here is not to analyze the behaviour under extreme events but rather to establish whether typical ice conditions have any noticeable effect on the vibration patterns since this is important information for future health monitoring of the facility. As observed in Figure 2-4B, the frequency content of the ambient

\footnotetext{
${ }^{1}$ Also in: Cheung MS, Lau DT, Li WC. Local vibration of girder box on Confederation Bridge. Internal report Ottawa-Carleton Bridge Research Institute, Carleton University.
} 
response in the presence of ice floes is not too dissimilar to the response under the ambient scenarios without ice. However, some subtle differences can be observed. First, the energy content in the $9-17 \mathrm{~Hz}$ range appears to be higher for the "ambient with ice" scenarios as compared to the other scenarios. This may be attributable to the existence of higher frequency components in the dynamic ice forces resulting from the various mechanisms of brittle fracturing and friction of the ice against the piers. Second, as compared to the "ambient" scenarios, the peak frequencies are different. However, this difference may just be due to the slightly higher wind speeds of the "ambient" datasets in this study. Third, some vibration modes in the vertical direction of the response, such as the one at $4.16 \mathrm{~Hz}$, are more clearly excited under the loading scenarios with ice floes than under the other loading scenarios. The most important vibration modes under the "ambient with ice" scenarios are observed at $1.8 \mathrm{~Hz}, 2.79 \mathrm{~Hz}$ and $3.83 \mathrm{~Hz}$ in the vertical direction, and $0.48 \mathrm{~Hz}, 0.36 \mathrm{~Hz}$ and $3.3 \mathrm{~Hz}$ in the lateral direction.

\subsection{Data analysis}

\subsubsection{Data processing}

The handling of the monitoring data from collection, transmission and archival to engineering interpretation includes tasks such as data management, data processing, and data analysis. The following processing tasks are performed to prepare the data for system identification analysis.

- Baseline adjustment of the acceleration time histories by removing any voltage drift from the accelerometer signals.

- Low-pass filtering with a cut-off frequency of $9 \mathrm{~Hz}$ using a Chebyshev type II filter of order 12. This eliminates high frequency noises and signal components, 
including those corresponding to the local distortional vibration of the box girder, enhancing the response signals in the band of interest $(0-5.5 \mathrm{~Hz})$. The filtering is carried out in the forward and reverse directions to eliminate nonlinear phase distortion. The low-pass filtering also serves as an anti-aliasing filter for the subsequent re-sampling of the data.

- Down-sampling of the data to one third of the original sampling rate. The result is a substantial reduction in data size without any significant loss of resolution.

The above processing tasks, as well as the system identification analysis of the data, are carried out using monitoring software developed for the Confederation Bridge project by Desjardins et al. (2006).

\subsubsection{System identification}

For large scale civil structures like the Confederation Bridge, it tends to be impractical to carry out forced vibration measurements which have been the common practices and considered as the most reliable means for vibration based evaluation of mechanical engineering structures. Consequently, operational loadings are relied upon as the input excitations in the dynamic response measurements of the Confederation Bridge. Since it is practically impossible to measure the operational forces, output-only system identification techniques are required in the system identification analysis of such vibration response data. Among the different system identification techniques proposed for civil engineering monitoring applications the stochastic subspace identification (SSI) method is a reliable output-only identification technique which compares favourably to other available methodologies (Peeters and Ventura 2003). The SSI algorithm used in the present study is summarized below. For a more in-depth look at the SSI method the reader may refer to the work by Peeters and De Roeck (1999) or Peeters (2000). 
The structural dynamics and the data observed from it are represented by the following discrete-time stochastic state-space model

$$
\begin{aligned}
& \mathbf{x}_{k+1}=\mathbf{A} \mathbf{x}_{k}+\mathbf{w}_{k} \\
& \mathbf{y}_{k}=\mathbf{C} \mathbf{x}_{k}+\mathbf{v}_{k}
\end{aligned}
$$

in which $\mathbf{x}_{\mathrm{k}}$ is an $n \times 1$ vector describing the system state (displacement and velocity) at the instant $k \Delta t$, where $\Delta t$ is the sampling interval, $\mathbf{y}_{\mathrm{k}}$ represents the measurements or outputs, $\mathbf{A}$ is the discrete state matrix containing the system eigenvalues and describing the dynamics of the system, $\mathbf{C}$ is the discrete output matrix that relates the system state to the measured outputs, while $\mathbf{w}_{\mathrm{k}}$ and $\mathbf{v}_{\mathrm{k}}$ account for the immeasurable inputs, which are assumed to be white, zero-mean stochastic processes, independent of the state vector. Measured outputs are typically accelerations, but may also be displacements, velocities. For the Confederation Bridge the measured outputs are the acceleration responses.

Under the assumptions of stationarity of the state vector and whiteness of the stochastic input terms it can be shown that output correlation matrices $\mathbf{R}_{i}$, defined as $\mathbf{R}_{i}=\mathrm{E}\left[\mathbf{y}_{k+i} \mathbf{y}_{k}^{T}\right]$, can be factorized into a triplet containing the system matrices as follows,

$$
\mathbf{R}_{i}=\mathbf{C A}^{i-1} \mathbf{G}
$$

where $\mathbf{G}$ is defined as $\mathbf{G}=\mathrm{E}\left[\mathbf{x}_{k+1} \mathbf{y}_{k}^{T}\right]$. Equation (2-2), which is analogous to the factorization of impulse response functions in input-output modal analysis, is the cornerstone of the stochastic subspace method. By taking advantage of this factorization property, correlation matrices are first estimated from the data and then decomposed to obtain the system matrices, ultimately yielding the eigenvalues and eigenvectors, as is briefly summarized below. 
Based on an ergodicity assumption, estimates of the output correlation matrices can be obtained as

$$
\hat{\mathbf{R}}_{i}=\frac{1}{N} \sum_{k=0}^{N-1} \mathbf{y}_{k+i} \mathbf{y}_{k}^{T}
$$

where $N$ is the number of samples. Data correlations offer the advantages of significantly compressing the data while preserving the modal information and eliminating uncorrelated noise.

In experimental modal analysis applications, typically many sensors are used to measure the response of the structural system in order to obtain the mode shapes with a reasonable spatial resolution. As a result, there is usually some redundancy in the data because all sensors carry essentially the same information regarding the frequency and damping ratios, except if they are located at modal nodes. Taking advantage of this observation, computations can be substantially reduced by computing data correlations with respect to just a small subset of reference sensor channels. Reference output correlation matrices computed at different time lags are gathered into a block Toeplitz matrix as follows

$$
\mathbf{T}_{1 \mid i}^{r e f}=\left[\begin{array}{cccc}
\mathbf{R}_{i}^{r e f} & \mathbf{R}_{i-1}^{r e f} & \ldots & \mathbf{R}_{1}^{r e f} \\
\mathbf{R}_{i+1}^{r e f} & \mathbf{R}_{i}^{r e f} & \ldots & \mathbf{R}_{2}^{r e f} \\
\ldots & \ldots & \ldots & \ldots \\
\mathbf{R}_{2 i-1}^{r e f} & \mathbf{R}_{2 i-2}^{r e f} & \ldots & \mathbf{R}_{i}^{r e f}
\end{array}\right]
$$

where $\mathbf{R}_{i}^{r e f}=\mathrm{E}\left[\mathbf{y}_{k+i}\left(\mathbf{y}_{k}^{r e f}\right)^{T}\right], \mathbf{y}_{k}^{\text {ref }}$ is the $r \times 1$ vector of sampled reference responses at time instant $k, r$ is the number of reference channels, and $i$ is a parameter that defines the portion of the data correlations - i.e. the number of time lags - to be used for system identification. Mathematically, this parameter must satisfy the relation $r \times i>n$, where $n$ is the order of the state-space model. Based on Eq. (2-2), the block Toeplitz matrix can be expressed as follows 


$$
\mathbf{T}_{1 \mid i}^{r e f}=\left[\begin{array}{c}
\mathbf{C} \\
\mathbf{C A} \\
\ldots \\
\mathbf{C A}^{i-1}
\end{array}\right]\left[\begin{array}{llll}
\mathbf{A}^{i-1} \mathbf{G}^{r e f} & \ldots & \mathbf{A G}^{r e f} & \mathbf{G}^{r e f}
\end{array}\right]=\mathbf{O}_{i} \boldsymbol{\Gamma}_{i}^{r e f}
$$

These two factors are known as the extended observability and reversed extended stochastic controllability matrices, respectively. Estimates of these two factors are obtained via the Singular Value Decomposition (SVD) of the block Toeplitz matrix.

$$
\mathbf{T}_{1 i i}^{r e f}=\mathbf{U S V}^{T}=\left[\begin{array}{ll}
\mathbf{U}_{1} & \mathbf{U}_{2}
\end{array}\right]\left[\begin{array}{cc}
\mathbf{S}_{1} & \mathbf{0} \\
\mathbf{0} & \mathbf{0}
\end{array}\right]\left[\begin{array}{c}
\mathbf{V}_{1}^{T} \\
\mathbf{V}_{2}^{T}
\end{array}\right]=\mathbf{U}_{1} \mathbf{S}_{1} \mathbf{V}_{1}^{T}
$$

where $\mathbf{S}_{1}$ is a diagonal matrix containing singular values in descending order. Using equations (2-5) and (2-6), the following so-called internally balanced realization may be obtained

$$
\begin{aligned}
& \mathbf{O}_{i}=\mathbf{U}_{1} \mathbf{S}_{1}^{1 / 2} \\
& \boldsymbol{\Gamma}_{i}^{r e f}=\mathbf{S}_{1}^{1 / 2} \mathbf{V}_{1}^{T}
\end{aligned}
$$

Since the true model order of the system is typically unknown and difficult to determine, solutions of increasing model orders, 1 to $n_{\max }$, are obtained by taking an increasing number of singular values from the SVD to construct the estimates of $\mathbf{O}_{i}$ and $\boldsymbol{\Gamma}_{i}^{\text {ref }}$. At each model order, system matrices $\mathbf{A}$ and $\mathbf{C}$ are extracted from $\mathbf{O}_{i}$ and $\boldsymbol{\Gamma}_{i}^{r e f}$. Then, an eigenvalue decomposition of A yields the system's complex eigenvalues and vectors as follows

$$
\mathbf{A}=\boldsymbol{\Psi} \Lambda \boldsymbol{\Psi}^{-1}
$$

where $\Psi$ is a matrix of the complex eigenvectors and $\Lambda$ is a diagonal matrix containing the discrete-time eigenvalues $\mu_{i}$, which are directly related to the continuous time eigenvalues $\lambda_{j}$ that contain the modal frequencies $\omega_{j}$ and damping ratios $\xi_{j}$

$$
\lambda_{j}=\frac{\log _{e}\left(\mu_{j}\right)}{\Delta t}=-\xi_{j} \omega_{j}+i \sqrt{1-\xi_{j}^{2}} \omega_{j}
$$


It should be noted that the frequency resolution of the modal frequencies identified by the SSI method is not limited by the duration of the data recordings as in the traditional frequency domain techniques.

Finally, the observed part of the mode shapes are obtained from the eigenvectors as follows

$$
\mathbf{V}=\mathbf{C \Psi}
$$

Although theoretically the order of the system $(n)$ can be determined by looking at the number of non-zero singular values of the SVD, when analyzing the data from real structures the model order is typically masked by the noise in the data. The noise may arise from a combination of the following sources

- Modeling approximations

- Measurement inaccuracies

- The use of finite datasets to compute estimates of the correlations

- Non-stationarity and non-linearity behaviour in the data

To overcome the problem of the unknown and hidden system order, a technique known as stabilization diagrams can be employed. In this technique, the set of models of different order $\left(1<n<n_{\text {max }}\right)$ identified by taking a different number of singular values and vectors from the SVD are presented in a diagram where the distinction between "true" or stable system solutions and "spurious" solutions caused by the noise becomes apparent to the analyst. A typical stabilization diagram is shown in Figure 2-5.

In the present study, a relatively high maximum model order $n_{\max }=150$ is used in the construction of the stabilization diagrams to allow for a clear visualization of stabilized trends. The actual model order of the data analyzed here is typically lower than this, typically around 40 , but may vary between different datasets depending on the number of modes 
being excited. The number of time lags of the data correlations used in the construction of the Toeplitz matrix (Eq. (2-4)), which forms the basis of the system identification, is taken as $i=200$ for all datasets analyzed. This value of $i$ not only satisfies the mathematical requirement of $r \times i>n_{\max }$ but also ensures that the data correlations of the Toeplitz matrix span more than a full vibration cycle of the lowest mode. From the authors' experience, this criterion is important as it ensures there is enough information from the lowest modes in the data correlations to ensure enough accuracy in their identification.

As mentioned, data correlations computed with respect to just a subset of wellselected reference sensors can be used in the SSI algorithm instead of using the crosscorrelations of all sensors in order to reduce the computational burden without significant loss of accuracy. In this study, the four vertical and two lateral sensors at monitoring locations 7 and 9 (Figure 2-1(b)) are used. These sensors are suitable references because of their relatively high response amplitudes and because their locations do not simultaneously coincide with modal nodes of any of the important vibration modes of the bridge.

\subsection{Finite element models}

Two different finite element models of the Confederation Bridge, a shell element model and an equivalent beam element model, are constructed using COSMOS finite element software to obtain the theoretical dynamic behaviour of the bridge for the purpose of verifying whether or not the expected design behaviour corresponds well to the behaviour observed in the field. The two different computer models of the bridge are constructed with slightly different modeling objectives to better simulate different aspects of the dynamic behaviour of the bridge. In particular, the shell element model is constructed mainly to model the purely torsional vibration modes of the bridge as well as the local distortional 
modes of the girder, while the beam model better models the interaction between adjacent bridge spans.

The elastic modulus of the concrete is among the main structural and modeling parameters. It determines the overall stiffness of the bridge and has a direct effect on the static and dynamic behaviours of the structure. The elastic modulus as well as the strength of the concrete increase gradually with time as the concrete matures over the first few years of service. Hence, prior to comparison with experimental values, the finite element models must be updated to reflect the current conditions. To this end, modal analysis of the finite element bridge models is carried out using two different values of the Young's modulus $E_{c}$ of concrete. The first value of $E_{i}$ is the nominal 28-day design value of $E_{c 28}=35 \mathrm{GPa}$, and the second value of $E_{c}$ is based on research by the materials and deflections monitoring group of the project from tests on samples from the actual concrete used in the Confederation Bridge (Ghali et al. 2000).

The target 28-day strength of concrete in the design specifications was $\mathrm{f}_{628}=55 \mathrm{MPa}$ and the corresponding target elastic modulus was $E_{i 28}=35 \mathrm{GPa}$. Ghali et al. (2000) tested 24 concrete cylinders taken from two Confederation Bridge batches. The cylinders were tested at the ages of $2,7,14,28,90$, and 180 days. The actual average measured value of $f_{c 28}^{\prime}$ was 63 $\mathrm{MPa}$ and $E_{c 28}$ was approximately $38.7 \mathrm{GPa}$. Based on the measurements, a best fit equation for the time-dependent variation of $E_{c}$ was proposed as follows

$$
E_{c}(t)=E_{c 28} \sqrt{e^{0.25\left(1-\sqrt{\frac{28}{t}}\right)}}
$$

in which $E_{c}(t)$ is the elastic modulus of concrete at age $t$, in days, and $E_{c 28}$ is $38.7 \mathrm{GPa}$, approximately the average measured value. The constant of 0.25 in the equation is for normal hardening cement. The 1000 -day value of $E_{c}$ given by this expression is $42.9 \mathrm{GPa}$. 
In the present study, two values of $E_{c}$ are used in the bridge finite element models for comparison purpose: the target 28-day value of $35 \mathrm{GPa}$ and a 3-year value of $43 \mathrm{MPa}$, which is expected to more realistically reflect the properties of the concrete of Confederation Bridge.

\subsubsection{Shell model}

A typical bridge portal frame corresponding to the instrumented portion of the structure between Piers 31 and 32 is modeled using shell elements. Four-node quadrilateral thin shell elements and three-node triangular shell elements are used, except for the ice shield component of the pier which is modeled by 4-node tetrahedron solid elements. The interaction of the portal frame with the connecting drop-in spans, which are not included in the model, is modeled by means of lumped mass elements. The mass of the drop-in span is equally divided and added to each bearing in the lateral and vertical directions, since all four bearings of a drop-in span prevent motion in these directions. The entire mass of the drop-in span is added in the longitudinal direction to the bearing which prevents longitudinal motion since only one of the four bearings of the drop-in span prevents relative motion in this direction. The dimensions and material properties of this model are based on the design drawing dimensions and specifications. The model has a total of 2381 elements and 2206 nodes, resulting in a relatively fine mesh, as shown in Figure 2-6(a).

\subsubsection{Beam model}

To gain a better understanding of the overall dynamic behaviour of the Confederation Bridge, a portion of the bridge between piers 29 and 32 including two consecutive portal frames plus the expansion drop-in span between them, are modeled using 3D beam elements, as shown in Figure 2-6(b). The chief objective of the beam model of the 
bridge is to model the interaction behaviour between adjacent portal frame units. In total, the beam model comprises 112 beam elements, 6 lumped mass elements and 113 nodes. End release codes of the beam elements are applied to model the hinge and sliding plate connections between the expansion drop-in span and the portal frames. The interactions with other adjacent drop-in spans at the boundary end-points of the girders are modeled by means of lumped mass elements. Half of the mass of the drop-in span is added at the boundary node in the lateral and vertical directions for the case of sliding plate bearings, while the entire mass of the drop-in is added to the boundary node in the longitudinal direction in the case of a flxed hinge bearing. The geometrical properties of the elements, such as cross-sectional area, moments of inertia and torsional constant are determined from the design drawings. For each structural component, such as girder, pier shaft, pier base and drop-in span, the mass density is calculated from the weight of the component and its volume in the model. The mass of significant non-structural components, such as the road barriers and pavement are included as additional mass density of the beam elements.

\subsection{Analysis results from monitoring data}

Modal vibration frequencies, mode shapes and damping ratios obtained by system identification analysis of the twenty-one monitoring datasets described earlier are presented here. The results are compared to the theoretical design values based on the finite element models for verification of the expected dynamic behaviour and material properties.

\subsubsection{Modal frequencies}

Table 2-2 presents a summary of the modal frequencies extracted from the monitoring data and the corresponding values from the beam and shell finite element models. 
Finite element model results based on the two different values of elastic modulus of concrete are compared to the identified frequencies. As discussed earlier, the specified nominal 28days design value of $E_{c}=35 \mathrm{GPa}$ is used as well as the 1000 -day value of $E_{c}=43 \mathrm{GPa}$, based on the monitoring of the actual concrete properties In general, the identified modal vibration frequencies are in reasonable agreement with the frequencies expected from the design of the bridge, with better overall agreement for the updated modulus of $E_{c}=43 \mathrm{GPa}$. On average, the measured frequencies are higher than the FE model frequencies based on the nominal modulus of elasticity of concrete $E_{c}=35 \mathrm{GPa}$, with an average difference of $15 \%$ for the beam model and $7 \%$ for the shell model. In contrast, the finite element modal frequencies based on the updated concrete modulus are much closer to the measured values with only a $4 \%$ average discrepancy for the beam model and $-3 \%$ for the shell model. The observation of the field measured modal frequencies being lower than the theoretical shell model results based on the updated concrete material modulus property correlates well with the usual observation of actual structures being typically less stiff than their finite element numerical models. Overall, the good agreement between the measured modal frequencies and the updated finite element model values shows that the structure's dynamic properties of stiffness and mass are reasonably close to the design values. The results also validate the dynamic monitoring data and the analysis techniques employed.

In Table 2-2, the column labelled "number of identifications" lists the number of monitoring datasets from which the corresponding eigenfrequencies and mode shapes have been identified, out of the total of 21 datasets considered. The numbers give an indication of how frequently each of the vibration modes is well excited under the typical loading scenarios and thus participates significantly in the measured structural responses. The relevance of this information is for practical structural condition assessment and damage 
detection based on continuous structural monitoring data, where not every theoretical vibration mode of the structure is available, but rather you are forced to rely only on the subset of modes which can be retrieved consistently from the monitoring data.

Table 2-2 also shows information regarding the variability of the modal frequencies extracted from the monitoring datasets. The values of standard deviations of the identified eigenfrequencies show that the variations in the extracted values can be significant. The variation in the identified frequencies relative to the mean appears to be higher for the lower frequency vibration modes and shows a decreasing trend for higher frequency modes. For the first eight modes below $1.0 \mathrm{~Hz}$, the average range of frequency variation is $9 \%$ of the mean, while higher modes have an average variation of $3 \%$ from the mean.

The variability among the results obtained from different datasets may be attributable to differences in the environmental conditions and loading scenarios at the times of data acquisition and also to measurement noise and deviations of the structural behaviour from modeling assumptions and computational errors. The sources and the practical implications of the different uncertainties in vibration monitoring data are discussed in more detail in Chapter 3.

In general, the uncertainty in the identified dynamic properties represents a major challenge for the use of vibration monitoring data for structural condition assessment. Changes in the dynamic properties of the structure caused by damage or deterioration could easily be masked by the normal variability of the data, especially at the early stages of damage that are of most practical interest. The observation of higher than average variability in the lower frequency vibration modes, which are often important in the vibration behaviour especially under the frequently occurring scenarios of high wind loading, could be an added difficulty for vibration based health monitoring of this kind of structures. 
It should be noted that the values of variability of modal parameters presented here, which are obtained from $90 \mathrm{~s}$ duration datasets, are only preliminary results. More accurate values of variability are presented in Chapter 3, where the accuracy of system identification is improved by using significantly longer recordings $(600 \mathrm{sec})$. The increase in the duration of the recordings improves the estimates of the data correlation functions, in Eq. (2-3)), which form the basis of the system identification algorithm. Still, the results presented above are fully relevant for the purpose of identifying the vibration modes normally excited under typical loading scenarios, for verifying the design dynamic properties and behaviour of the bridge, as well as giving an initial overview of the variability behaviour.

\subsubsection{Mode shapes}

Figure 2-7 shows the vibration mode shapes extracted by system identification from the field monitoring data and a comparison with the corresponding expected design mode shapes obtained from the finite element models. It should be noted that not all of the identified modes are found to have a theoretical counterpart. This is partly because the finite element models only cover a limited portion of the bridge. The beam finite element model is used as the main reference with the exception of the torsional modes which are compared to the results from the shell finite element model. The overall agreement of the identified mode shapes with the expected mode shapes is good, especially considering the complexity of the structure and the high levels of noise and uncertainty associated with output-only system identification from vibration monitoring data. As may be observed from Figure 2-7, discrepancies between the identified and theoretical mode shapes tend to be relatively larger at the expansion drop-in span, probably as a result of the complexity of the actual drop-in span support conditions. The actual drop-in span is supported on four independent bearings, three of which are unidirectional sliding plates and the fourth is a fixed hinge pot bearing. 
These supports are modeled using end-release codes in the beam finite element model, which are basically equivalent to hinge and roller idealizations. The asymmetry and frictional restraints of the actual bearings, which are not accounted for in the finite element model, lead to non-classical damping and phase delays in the identified mode shapes, and may be partly responsible for the discrepancies observed.

A numerical estimate of the quality of the correlation between identified mode shapes and their finite element counterparts representative of the expected design behaviour, can be obtained using the Modal Assurance Criterion (MAC)

$$
\operatorname{MAC}\left(\boldsymbol{\Psi}_{i}, \boldsymbol{\Psi}_{j}\right)=\frac{\left|\boldsymbol{\Psi}_{i}^{*} \boldsymbol{\psi}_{j}\right|^{2}}{\left(\boldsymbol{\Psi}_{i}^{*} \boldsymbol{\Psi}_{i}\right)\left(\boldsymbol{\psi}_{j}^{*} \boldsymbol{\psi}_{j}\right)}
$$

where $\psi_{i}$ and $\psi_{j}$ are the two different mode shape vectors being compared and the symbol “* indicates complex conjugate transpose (Ewins 1984). The MAC is a normalized projection of one mode vector onto the other, with a value of 1 indicating perfect correlation and a value of 0 indicating no correlation. MAC values between extracted and theoretical mode shapes are presented in Table 2-3. These values are computed between the mean of normalized extracted mode shapes and the corresponding theoretical mode shape. For the computation of the mean extracted mode shape, the mode shapes extracted from different datasets are normalized by their maximum coefficient. For the beam finite element model, 7 out of the 18 modes with experimental counterparts have MAC values around 0.9 indicating good correlation. The average MAC value for extracted vs. theoretical beam model mode shapes is lower at 0.74 . For the shell model, 9 of the 14 modes with experimental counterparts have MAC values close to or above 0.9 and the average MAC value is 0.79 , indicating that this more realistic model is able to capture the behaviour of the structure more accurately. Even though it is recognized that the MAC values obtained leave some 
room for optimization of the models via techniques such as finite element model updating, the overall agreement between extracted mode shapes and the expected shapes from the finite element models is good enough to indicate that the dynamic behaviour is as expected from design within a reasonable margin of tolerance.

Table 2-3 also presents average MAC values for the extracted mode shapes, which give a measure of the consistency of the mode shapes extracted from the different monitoring datasets. The average MAC value for different identifications of the same vibration mode is computed as follows

$$
\overline{M A C}=\frac{\sum_{i=1}^{M} M A C\left(\bar{\Psi}, \boldsymbol{\psi}_{i}\right)}{M}
$$

where $\bar{\psi}$ is the mean of normalized extracted mode shapes of the given vibration mode and $M$ is the number of identifications. As noted earlier, vibration modes are generally not identifiable from all monitoring datasets. Table 2-2 gives the number of identifications for each mode. In general, the average MAC values of Table 2-3 show that there is reasonable consistency of the mode-shapes identified from the different datasets. Average MAC values for extracted mode shapes are above 0.8 for 24 of the 25 vibration modes, while 15 are equal or above 0.9 . The only mode with average MAC lower than 0.8 is the 3.3 torsional mode. This is because this mode becomes easily coupled with its close vertical counterparts.

While the consistency in the identified mode shapes might be considered satisfactory in the traditional civil engineering sense, where differences of $5 \%$ or $10 \%$ tend to be tolerable, it should be recognized that if the identified mode shapes are to be used for structural condition assessment, i.e. to detect structural damage or deterioration from changes in the mode-shapes, then the level of variability observed among the different identifications would probably cause difficulties. The reason is that mode shapes, and modal parameters in general, 
typically have very low sensitivities to damage, so the changes caused by damage or deterioration tend to be very small. As a result, the variations among different identifications of the same mode could easily be comparable or larger than the changes due to damage, making it difficult to detect and locate damage based on mode shape changes.

To take effective measures to minimize the variability and help overcome this challenge in the practical application of structural health monitoring, it is first important to understand the sources of uncertainty in the parameters extracted from the monitoring data, as discussed later on in Chapter 3. At the same time, approaches of condition assessment based on statistical techniques designed to work in the presence of relatively large uncertainties in the data, such as novelty detection and pattern recognition techniques are promising and have shown good results in recent studies (e.g. Ko et al. 2002, Haritos and Owen 2004).

\subsubsection{Modal damping ratios}

The averages and standard deviations of the damping ratios of the vibration modes identified from the field monitoring datasets are presented in Table 2-3. The overall average modal damping ratio, considering all datasets and all identified vibration modes, is $1.3 \%$. It is worth noting that the mean extracted modal damping ratios show a slight decreasing trend with increasing frequency (Figure 2-8). The modal damping ratios for modes below $2.0 \mathrm{~Hz}$ are between $1.4 \%$ and $2.1 \%$ (with only two exceptions) while those for modes between 2.0 $\mathrm{Hz}$ and $5.2 \mathrm{~Hz}$ are between $0.4 \%$ and $1.3 \%$. Table $2-3$ gives the standard deviations from the mean for the damping ratios obtained from different datasets. As expected, the standard deviations obtained for the damping ratios are much higher than those obtained for the eigenfrequencies, with an average standard deviation of $54 \%$ from the mean values. The relatively large variation in the damping ratios is attributable to the complexity of damping 
phenomena, which are only roughly represented by the viscous damping assumption in the model of the structural system.

In comparing to expected design values, it should be recognized that the damping ratios assumed at the design stage differ according to the type of loading being considered. For wind loading, King and Davenport (1997) conducted wind-tunnel investigations using a full aero-elastic model of the Confederation Bridge and tested their model with two structural damping values, a low value of $0.13 \%$ was used in order to accentuate sensitivity of the structure to vortex shedding and galloping, and a value of $0.63 \%$, considered to be more representative of prestressed concrete structures, was used for the remainder of the tests. In light of the results identified from the monitoring data, the assumed value of $0.63 \%$ seems to be reasonably conservative compared to the identified values for the lower modes, which usually govern the wind-driven response.

To evaluate the dynamic effects of ice forces on the Confederation Bridge, Langohr and Ghali (1997) calculated the maximum bending moment at the base of a pier under simulated time varying ice forces using a simple two-degree-of-freedom model of the pier and also a more sophisticated space-frame model. A damping ratio of $5 \%$ was assumed for both of their analysis models, which is higher than the values extracted from the monitoring data in the present study. However, it is recognized that ice thickness at the time of measurement may have a significant effect on the damping ratios. Under heavy ice conditions more friction effects can be expected, and consequently the damping ratios are likely to be higher than the measured values reported here which are for relatively small ice drafts.

For the seismic design response spectra used in the calculation of earthquake induced forces on the bridge, structural damping ratios of both $2 \%$ and $5 \%$ were assumed Jaeger et al. 
1997). These values of damping are also somewhat higher than the average values extracted from the monitoring data. However, the assumed values may be considered reasonable given that damping can be expected to increase significantly as a result of the additional energy dissipation mechanisms that tend to develop in the structural responses associated to designlevel earthquake forces.

\subsection{Conclusions}

A study of the dynamic behaviour of the Confederation Bridge under different loading scenarios and a verification of its modal properties extracted by output-only system identification of vibration monitoring data against expected design values has been presented. A total of twenty-one field monitoring datasets collected at different times and under different loading scenarios have been used in the investigation.

First, the typical dynamic response patterns of the Confederation Bridge under different loading scenarios and environmental conditions are determined from spectral analysis of the selected monitoring datasets corresponding to the different loading scenarios, including high wind, heavy traffic, typical ambient conditions involving a mix of wind and traffic and ambient conditions in winter season with ice floes in the strait. The characteristic frequency content including dominant frequencies in the dynamic behaviour under each of these loading scenarios has been established, which is essential for use of the monitoring data for structural assessment and health monitoring of the bridge in the future.

From the output only system identification analysis of the monitoring data, twentyfive vibration modes are identified in the $0-5.2 \mathrm{~Hz}$ range. The extracted vibration modes are compared to the expected design values based on finite element models of the bridge constructed from design drawings and material specifications of the structure with the aim of verifying that the bridge behaves as expected from design. An updated nominal elastic 
modulus of the concrete, based on results from monitoring of the actual bridge concrete, is used in the finite element models to take into account the maturing of the material and its strength gain over time. Overall, the comparison to the updated models shows that the extracted dynamic properties of the bridge agree well with the expected design values. With the time-dependent material properties of the structure taken into account, the identified modal frequencies differ only by $-3 \%$ to $4 \%$ on average from the expected design values (the slight difference depends on the type of finite element model used to obtain the expected design values). The extracted mode shapes are reasonably similar to those expected from the design except for some localized discrepancies especially at the drop-in-span where the actual asymmetrical support conditions are slightly more complex than those assumed in the finite element models of this study. Damping ratio design assumptions, which vary according to the type of loading, have also been verified against modal damping ratios extracted from the monitoring data. The damping ratios assumed at the design stage for bridge response under wind seem to be reasonably conservative compared to the extracted values. Damping ratios of $2 \%$ and $5 \%$ assumed in the earthquake design are slightly higher than the extracted values, but also seem to be reasonable since significant additional damping can be expected in the large amplitude responses to strong ground motion. On the other hand, the data considered for this study, which included responses under light ice cover, is not considered appropriate to draw conclusions regarding the damping ratio assumptions for bridge responses under extreme-ice events.

The variability observed in the dynamic properties extracted by system identification of the field monitoring data is highlighted because it represents one of the main challenges for the use of the monitoring data for structural condition assessment given that the typically small changes in the extracted properties caused by damage or deterioration of the structure 
may well be obscured by the normal variability of the data. On average, identified modal frequencies exhibit a standard deviation of $1.8 \%$ from mean values while damping ratios exhibit significantly higher variability with an average standard deviation of $54 \%$ from the mean. The higher variability of the damping ratios stems from the complexity of the damping phenomena. Identified mode shapes also show some variability. The overall average MAC between different identifications of the same mode from different datasets is 0.88. The variability in the mode shapes is not uniform with some modes being more consistently identified than others. The most consistently identified modes have average $\mathrm{MAC}$ values close to 0.95 . Understanding the causes of the variability, together with the development of improved system identification and damage detection algorithms, is essential for the field application of vibration based health monitoring in the future. The issue of variability is explored further in the following chapter.

\subsection{Acknowledgements}

The development of the finite element models of the Confederation Bridge was carried out by Dr W.C. Li, former post-doctoral fellow of the Ottawa-Carleton Bridge Research Institute. Funding support and assistance provided to the monitoring project from NSERC through a CRD research grant, PWGSC, and Strait Crossing Bridge Ltd. (SCBL) are gratefully acknowledged. Technical information and assistance was provided by A. Solari and J. Egan of PWGSC and D. McGinn of SCBL. 
Table 2-1: Summary of vibration datasets

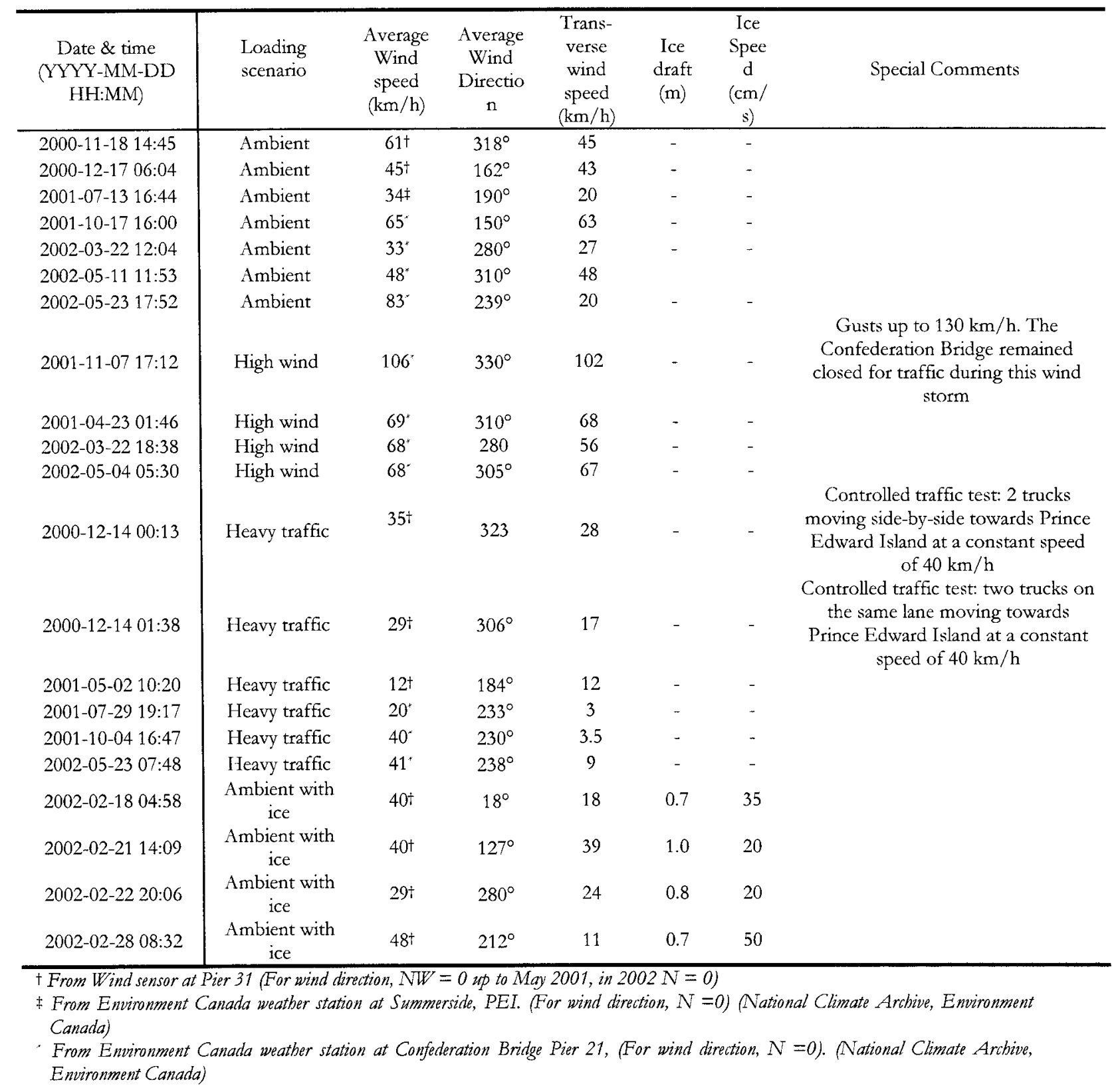


Table 2-2: Summary of modal frequencies from system identification of monitoring data and comparison to expected design values from finite element models

\begin{tabular}{|c|c|c|c|c|c|c|c|c|c|c|c|c|c|c|c|}
\hline \multirow{2}{*}{ 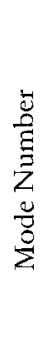 } & \multirow{2}{*}{ 志 } & \multirow{2}{*}{ 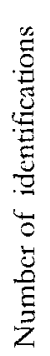 } & \multicolumn{5}{|c|}{ Extracted Frequency } & \multicolumn{4}{|c|}{ Theoretical Frequency } & \multicolumn{4}{|c|}{$\begin{array}{c}\text { Extracted - } \\
\text { Theoretical Frequency } \\
\text { Difference }\end{array}$} \\
\hline & & & $\frac{\sqrt{2}}{\sqrt[2]{2}}$ & $\frac{D}{2}$ & $\underset{\frac{B}{E}}{\stackrel{D}{z}}$ & 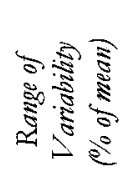 & 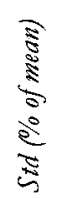 & 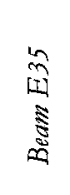 & 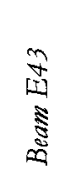 & 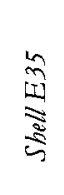 & 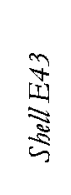 & 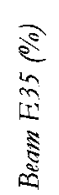 & $\frac{2}{i}$ & 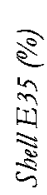 & $\begin{array}{l}\frac{2}{2} \\
\frac{\pi}{5} \\
\frac{\pi}{5}\end{array}$ \\
\hline 1 & $T$ & 6 & 0.34 & 0.32 & 0.37 & 16 & 5.8 & 0.28 & 0.31 & 0.32 & 0.35 & 22 & 10 & 6 & -4 \\
\hline 2 & $T$ & 14 & 0.47 & 0.46 & 0.48 & 5 & 1.4 & 0.46 & 0.51 & 0.49 & 0.54 & 2 & -8 & -3 & -12 \\
\hline 3 & $V$ & 5 & 0.57 & 0.52 & 0.63 & 19 & 6.8 & 0.47 & 0.52 & 0.49 & 0.55 & 21 & 9 & 15 & 4 \\
\hline 4 & $Y$ & 11 & 0.65 & 0.64 & 0.66 & 3 & 1 & 0.58 & 0.64 & 0.63 & 0.69 & 12 & 1 & 3 & -7 \\
\hline 5 & $V$ & 16 & 0.68 & 0.65 & 0.71 & 9 & 2.1 & 0.62 & 0.69 & -0 & $-{ }_{--}$ & 10 & -1 & - & $\begin{array}{l}-7 \\
--\end{array}$ \\
\hline 6 & $T$ & 2 & 0.89 & 0.87 & 0.91 & 5 & 3.6 & 0.84 & 0.93 & 0.88 & 0.98 & 6 & -4 & 1 & -9 \\
\hline 7 & $V$ & 17 & 0.92 & 0.89 & 0.95 & 7 & 2 & 0.79 & 0.88 & 0.86 & 0.95 & 16 & 5 & 7 & -4 \\
\hline 8 & $V$ & 8 & 0.97 & 0.95 & 0.99 & 4 & 1.5 & 0.82 & 0.91 & -- & -- & 18 & 7 & - & -- \\
\hline 9 & $T$ & 3 & 1.31 & 1.3 & 1.31 & 1 & 0.4 & 1.2 & 1.33 & 1.28 & 1.42 & 9 & -1 & 2 & -8 \\
\hline 10 & $V$ & 12 & 1.62 & 1.6 & 1.66 & 4 & 1.3 & $\ldots$ & - & 1.0 & 1.72 & -- & -1 & .. & -0 \\
\hline 11 & $V$ & 21 & 1.81 & 1.78 & 1.86 & 5 & 1.2 & 1.6 & 1.78 & 1.65 & $1 . \overline{82}$ & 13 & 2 & 10 & -1 \\
\hline 12 & $V$ & 20 & 2.73 & 2.7 & 2.79 & 3 & 0.9 & 2.34 & 2.59 & 1.00 & 1.02 & 17 & 5 & $\begin{array}{l}10 \\
--\end{array}$ & $\begin{array}{l}-1 \\
--\end{array}$ \\
\hline 13 & $V$ & 8 & 2.83 & 2.76 & 2.91 & 5 & 2 & 2.46 & 272 & 2.64 & 292 & 15 & 4 & 7 & -3 \\
\hline 14 & $V$ & 7 & 2.88 & 2.83 & 2.94 & 4 & 1.7 & .. & -- & -. & - & $\ldots$ & $\ldots$ & $\ldots$ & - \\
\hline 15 & $V$ & 4 & 3.07 & 3.03 & 3.11 & 3 & 1.1 & -- & -- & -- & -- & _- & $\ldots$ & $\ldots$ & - \\
\hline 16 & TP31-To & 6 & 3.3 & 3.26 & 3.34 & 3 & 1 & -- & - & 3.17 & 3.51 & - & - & 4 & -6 \\
\hline 17 & V.L & 4 & 3.31 & 3.29 & 3.35 & 2 & 0.9 & 2.73 & 3.03 & -- & - & 21 & 9 & -- & -- \\
\hline 18 & $V$ & 17 & 3.4 & 3.29 & 3.47 & 5 & 1.5 & -1. & - & -- & - & $\begin{array}{c}21 \\
--\end{array}$ & -- & -. & 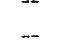 \\
\hline 19 & $V-L$ & 7 & 3.79 & 3.71 & 3.92 & 5 & 1.8 & 3.08 & 3.42 & 3.45 & 3.6 & 23 & 11 & 10 & 5 \\
\hline 20 & TP31-To & 4 & 4.08 & 4.03 & 4.16 & 3 & 1.5 & - & -- & 3.8 & 4.21 & - & -- & 7 & -3 \\
\hline 21 & $V-L$ & 4 & 4.11 & 4.07 & 4.16 & 2 & 0.9 & 3.49 & 3.87 & 3.62 & 4.01 & 18 & 6 & 13 & 2 \\
\hline 22 & V.L & 2 & 4.45 & 4.44 & 4.46 & 0 & 0.3 & 3.95 & 4.38 & -- & -- & 13 & 2 & -- & - \\
\hline 23 & $V \cdot \bar{L}$ & 5 & 4.71 & 4.66 & 4.74 & 2 & 0.6 & -- & -- & -- & -- & - & - & -- & -- \\
\hline 24 & $V$ & 3 & 4.91 & 4.83 & 4.99 & 3 & 1.7 & 4.34 & 4.81 & 4.52 & 5.01 & 13 & 2 & 9 & -2 \\
\hline 25 & $V$ & 8 & 5.13 & 5.03 & 5.2 & 3 & 1.3 & 4.43 & 4.91 & -- & -- & 16 & 5 & - & - \\
\hline & verages & 9 & & & & 4.8 & 1.8 & & & & & 15 & 4 & 7 & -3 \\
\hline
\end{tabular}

+ T: lateral, To: Torsional, V: Vertical, L: longitudinal, P31: pier 31 
Table 2-3: Summary of modal damping ratios and Modal Assurance Criterion values for vibration modes retrieved from monitoring data

\begin{tabular}{|c|c|c|c|c|c|c|c|c|c|}
\hline \multirow{2}{*}{ 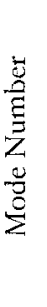 } & \multirow[b]{2}{*}{$\stackrel{\square}{E}$} & \multirow{2}{*}{ 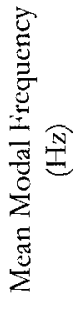 } & \multicolumn{3}{|c|}{ Modal Damping Ratios $(\xi)$} & \multicolumn{4}{|c|}{ Modal Assurance Criterion(MAC) } \\
\hline & & & $\stackrel{\Xi}{\Sigma}$ & 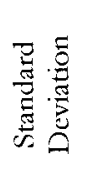 & 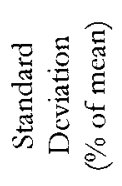 & 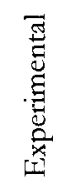 & 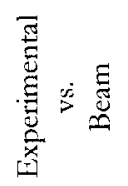 & 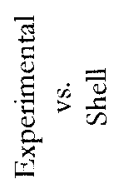 & 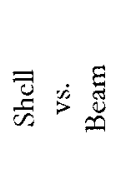 \\
\hline 1 & $T$ & 0.34 & 0.019 & 0.012 & 66 & 0.94 & 0.90 & 0.89 & 0.998 \\
\hline 2 & $T$ & 0.47 & 0.017 & 0.006 & 39 & 0.90 & 0.68 & 0.85 & 0.96 \\
\hline 3 & $V$ & 0.57 & 0.038 & 0.014 & 37 & 0.82 & 0.62 & 0.87 & 0.98 \\
\hline 4 & $V$ & 0.65 & 0.014 & 0.010 & 73 & 0.89 & 0.85 & 0.97 & 0.98 \\
\hline 5 & $V$ & 0.68 & 0.021 & 0.008 & 37 & 0.85 & 0.86 & -- & - \\
\hline 6 & $T$ & 0.89 & 0.021 & 0.017 & 84 & 0.93 & 0.36 & 0.62 & 0.98 \\
\hline 7 & $V$ & 0.92 & 0.015 & 0.007 & 42 & 0.91 & 0.88 & 0.98 & 0.98 \\
\hline 8 & $V$ & 0.97 & 0.016 & 0.007 & 42 & 0.92 & 0.86 & -- & - \\
\hline 9 & $T$ & 1.31 & 0.006 & 0.009 & 154 & 0.83 & 0.49 & 0.55 & 0.99 \\
\hline 10 & $V$ & 1.62 & 0.016 & 0.011 & 70 & 0.93 & -- & -- & -- \\
\hline 11 & $V$ & 1.81 & 0.015 & 0.008 & 53 & 0.94 & 0.91 & 0.96 & 0.995 \\
\hline 12 & $V$ & 2.73 & 0.008 & 0.004 & 52 & 0.91 & 0.90 & -- & -- \\
\hline 13 & $V$ & 2.83 & 0.013 & 0.007 & 57 & 0.92 & 0.79 & 0.89 & 0.99 \\
\hline 14 & $V$ & 2.88 & 0.01 & 0.006 & 59 & 0.89 & -- & -- & -- \\
\hline 15 & $V$ & 3.07 & 0.013 & 0.005 & 39 & 0.91 & -- & -- & $\ldots$ \\
\hline 16 & TP31-To & 3.3 & 0.006 & 0.003 & 48 & 0.56 & -- & 0.73 & -- \\
\hline 17 & $V-L$ & 3.31 & 0.009 & 0.004 & 50 & 0.92 & 0.70 & -- & -- \\
\hline 18 & $V$ & 3.4 & 0.008 & 0.004 & 50 & 0.90 & -- & -- & -- \\
\hline 19 & $V-L$ & 3.79 & 0.009 & 0.005 & 57 & 0.91 & 0.66 & 0.58 & 0.78 \\
\hline 20 & TP31-To & 4.08 & 0.004 & 0.001 & 37 & 0.86 & - & 0.41 & -- \\
\hline 21 & $V-L$ & 4.11 & 0.007 & 0.001 & 21 & 0.91 & 0.60 & 0.85 & 0.69 \\
\hline 22 & $V-L$ & 4.45 & 0.013 & 0.005 & 41 & 0.96 & 0.73 & -- & -- \\
\hline 23 & $V-L$ & 4.71 & 0.008 & 0.003 & 42 & 0.85 & -- & -- & -- \\
\hline 24 & $V$ & 4.91 & 0.01 & 0.006 & 63 & 0.89 & 0.77 & 0.92 & 0.99 \\
\hline 25 & $V$ & 5.13 & 0.008 & 0.004 & 48 & 0.86 & 0.75 & -- & -- \\
\hline \multicolumn{2}{|c|}{ Averages } & & 0.013 & 0.007 & 54 & 0.88 & 0.74 & 0.79 & 0.94 \\
\hline
\end{tabular}

$f$ T: lateral, To: Torsional, V: Vertical, L: longitudinal, P31: pier 31 


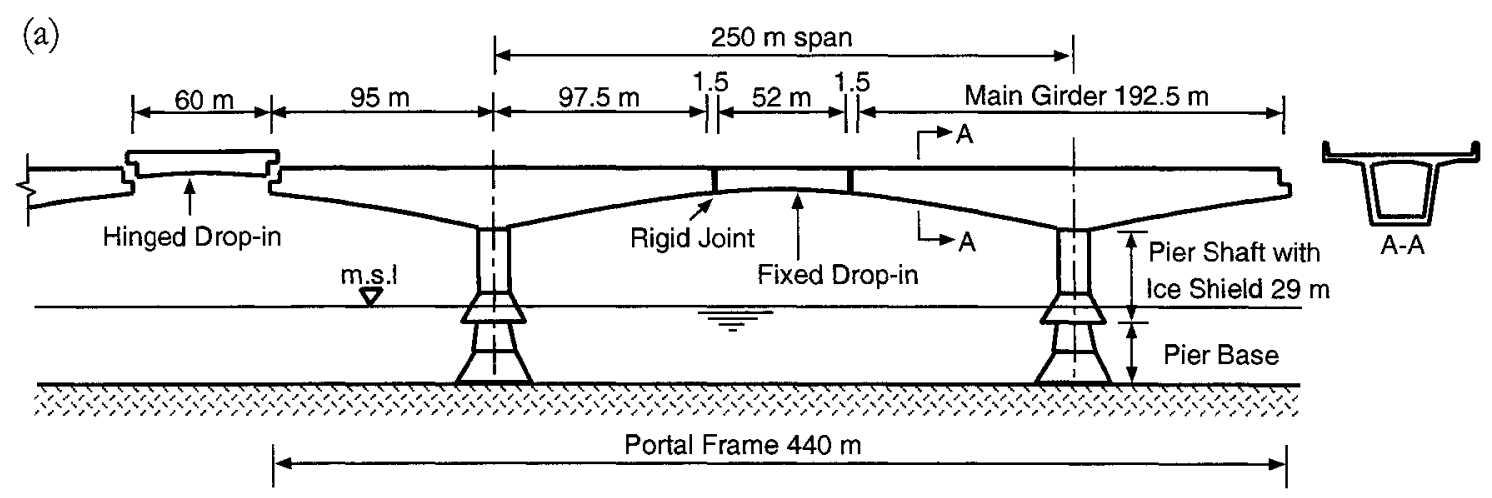

(b)

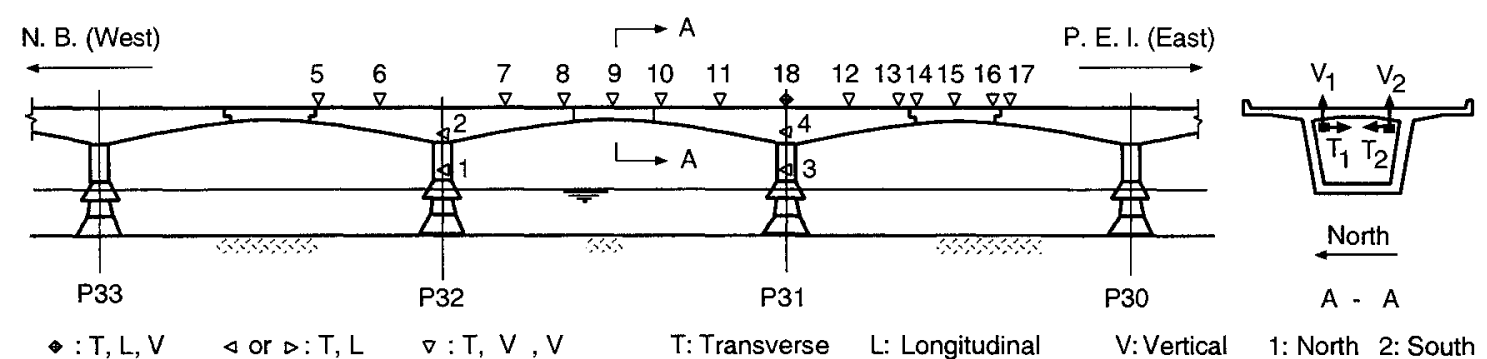

Figure 2-1: (a) Dimensions and main components of typical structural module; (b) Locations of accelerometers in Confederation Bridge monitoring system 


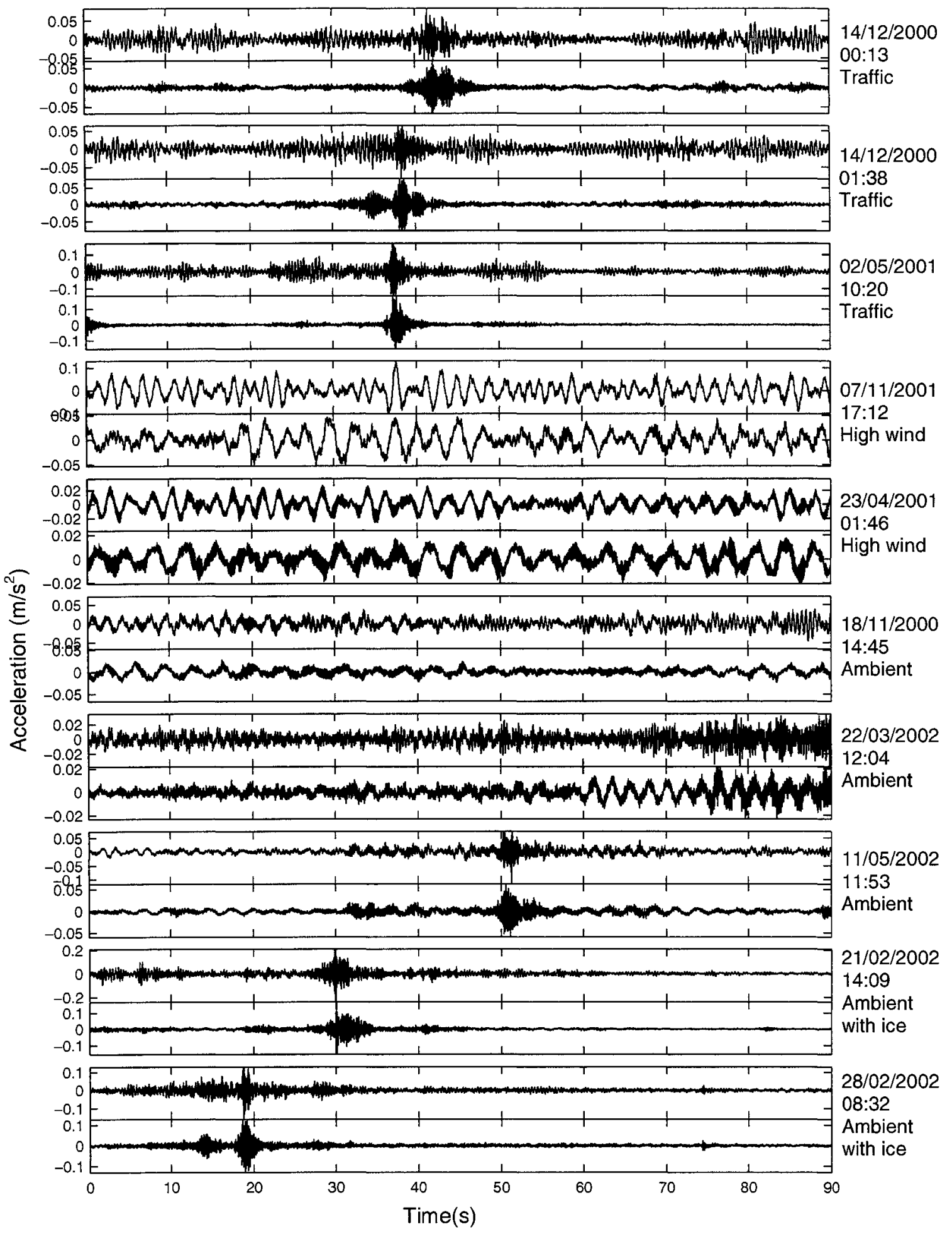

Figure 2-2: Vertical and lateral acceleration time histories at monitoring location 9 

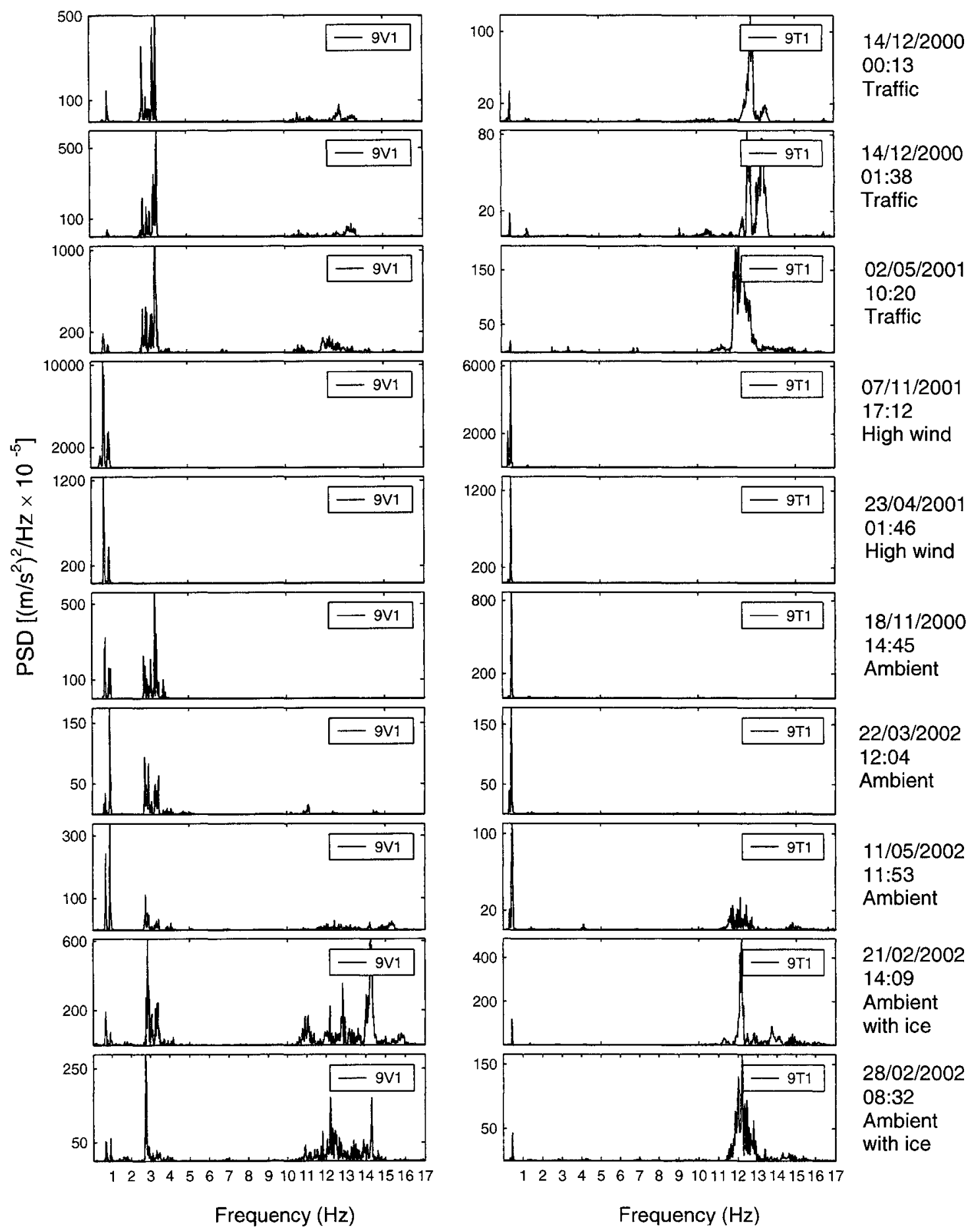

Figure 2-3: Vertical and lateral frequency content at monitoring location 9. 

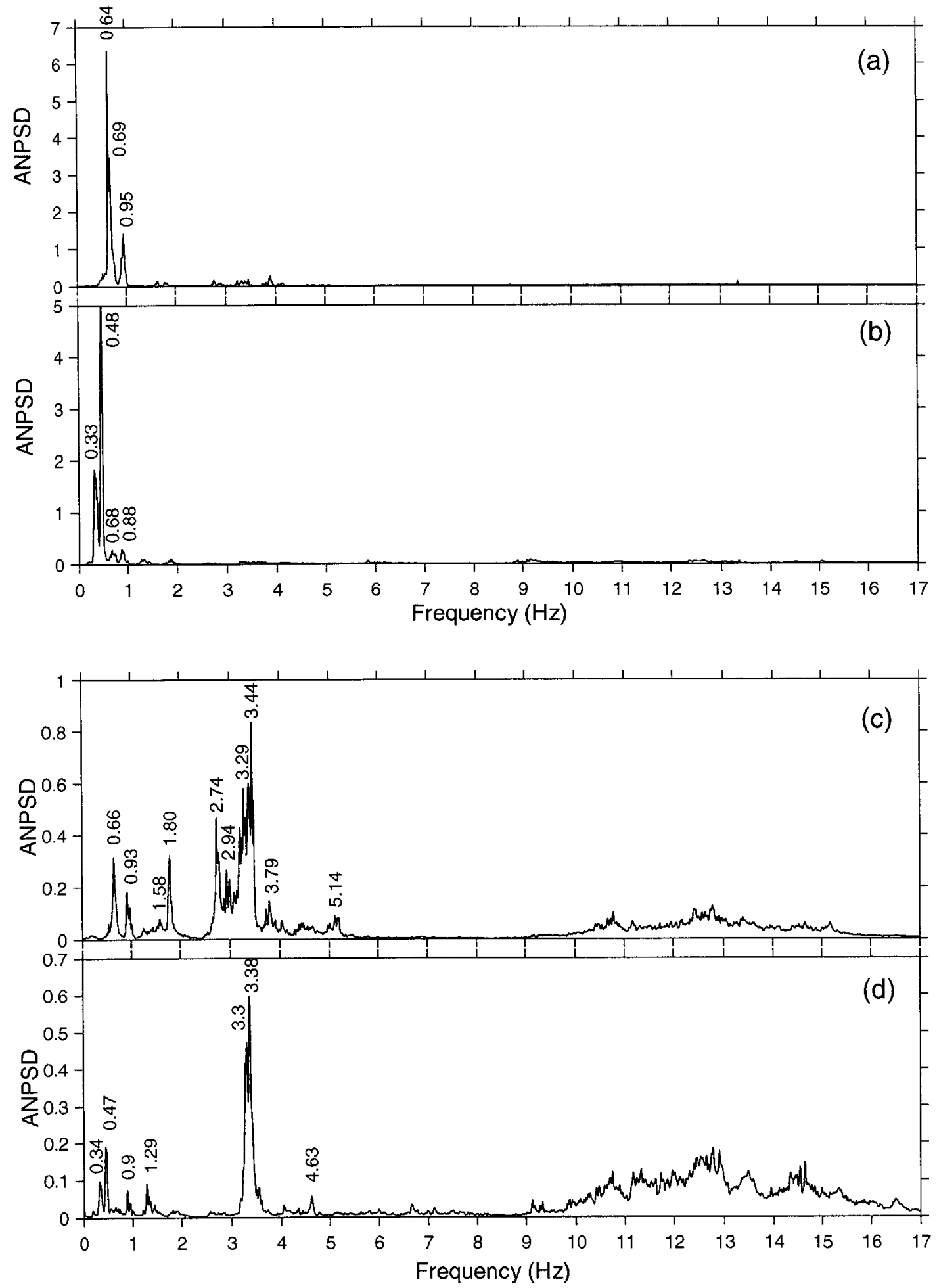

Figure 2-4A: ANPSDs of bridge response signals. (a) Wind loading scenarios vertical direction (b) wind lateral (c) traffic vertical (d) traffic lateral. 

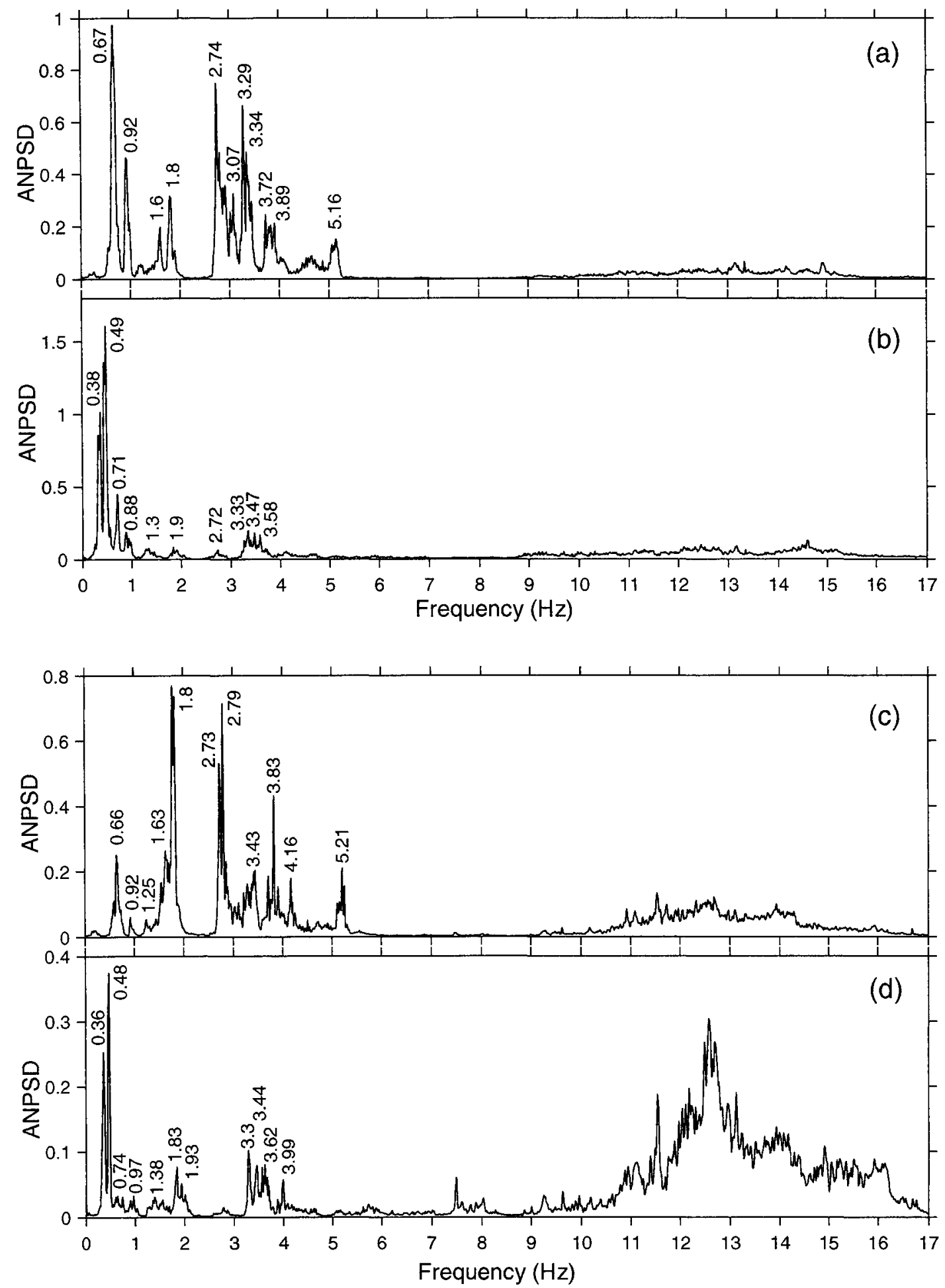

Figure 2-4B: ANPSDs of bridge response signals. (a) Ambient loading scenarios vertical direction (b) ambient lateral (c) ambient with ice vertical (d) ambient with ice lateral. 


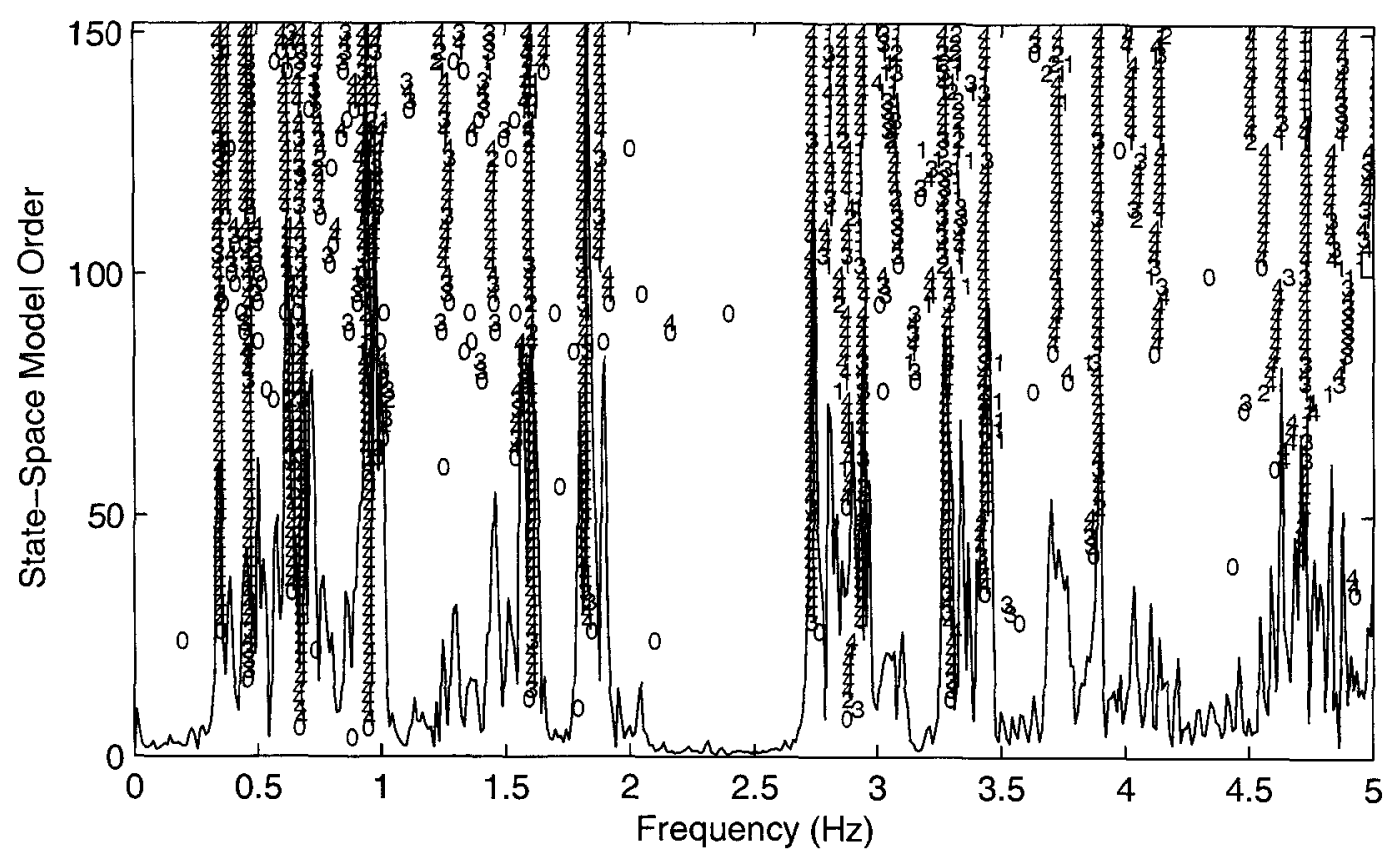

Figure 2-5: Typical stabilization diagram 


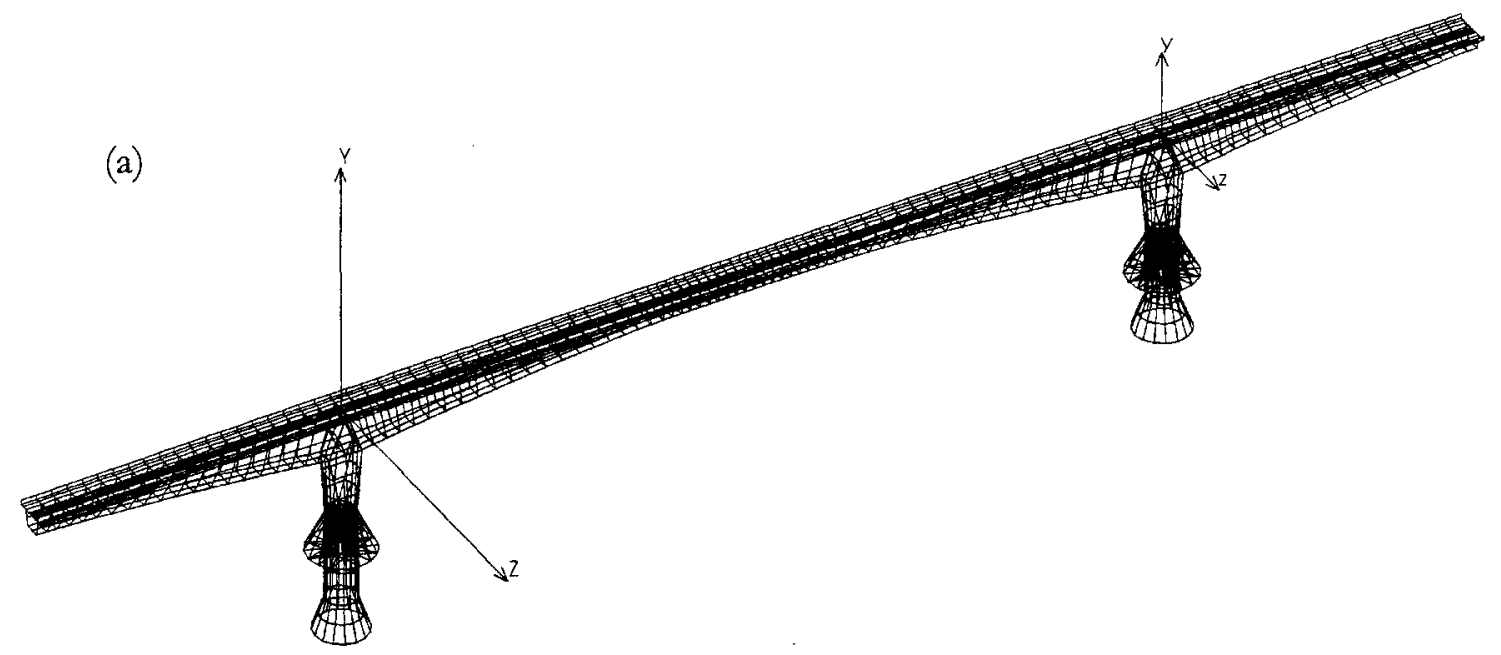

(b)
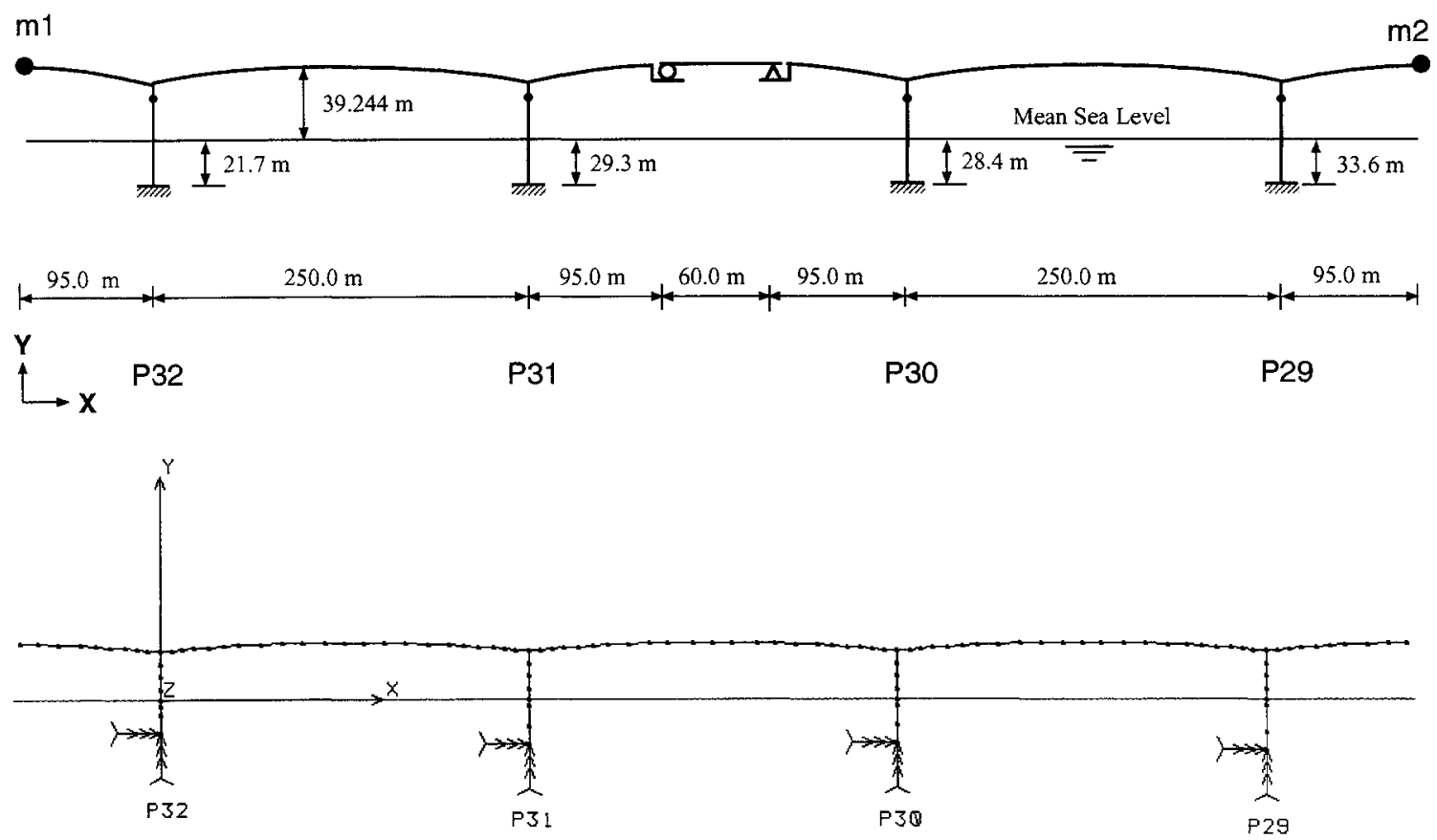

Figure 2-6: (a) Shell element model of Confederation Bridge; (b) Beam element model schematic and model showing mesh. 


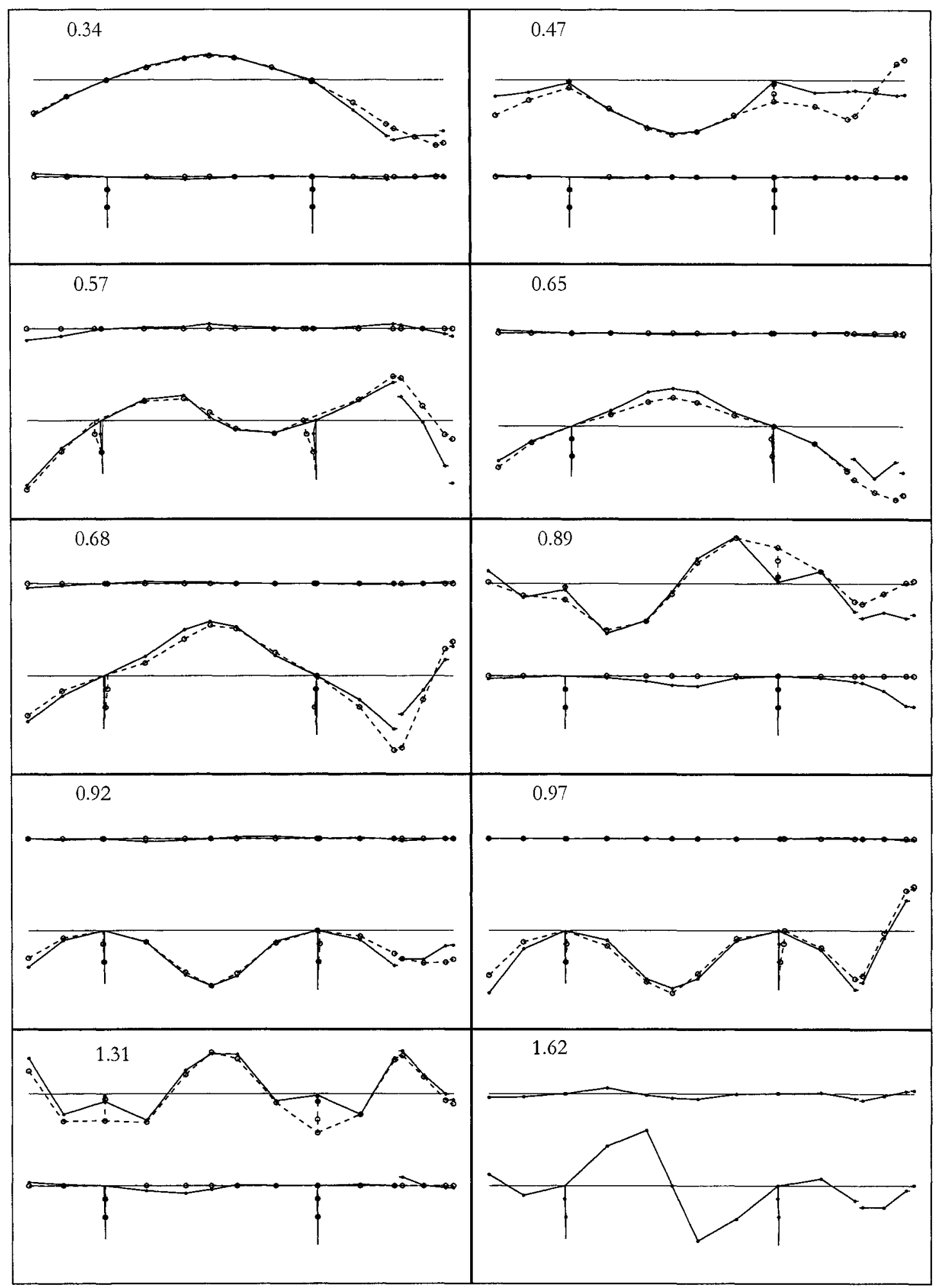

Figure 2-7A: Vibration mode shapes shown in top view and side view. Labels indicate mean extracted frequency. Markers indicate sensor locations. Extracted modes are shown in full line, full markers; theoretical shown in dotted line, open markers. 


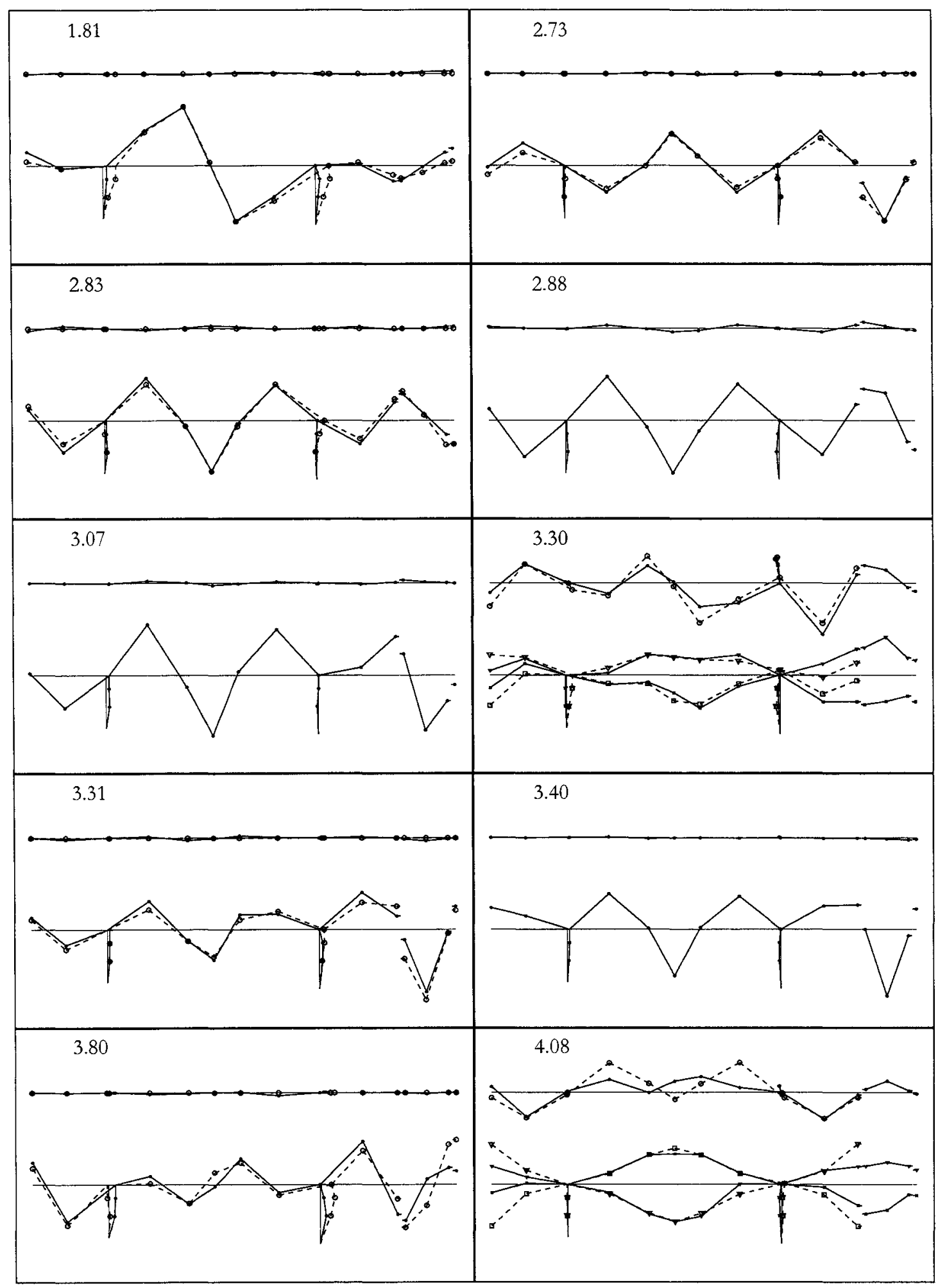

Figure 2-7B: Mode shapes shown in top view and side view. Labels indicate mean extracted frequency. Markers indicate sensor locations. Extracted modes are shown in full line, full markers; theoretical shown in dotted line, open markers. For torsional modes $(3.30 \mathrm{~Hz}$ and $4.08 \mathrm{~Hz})$ triangles indicate north side, squares south side. 


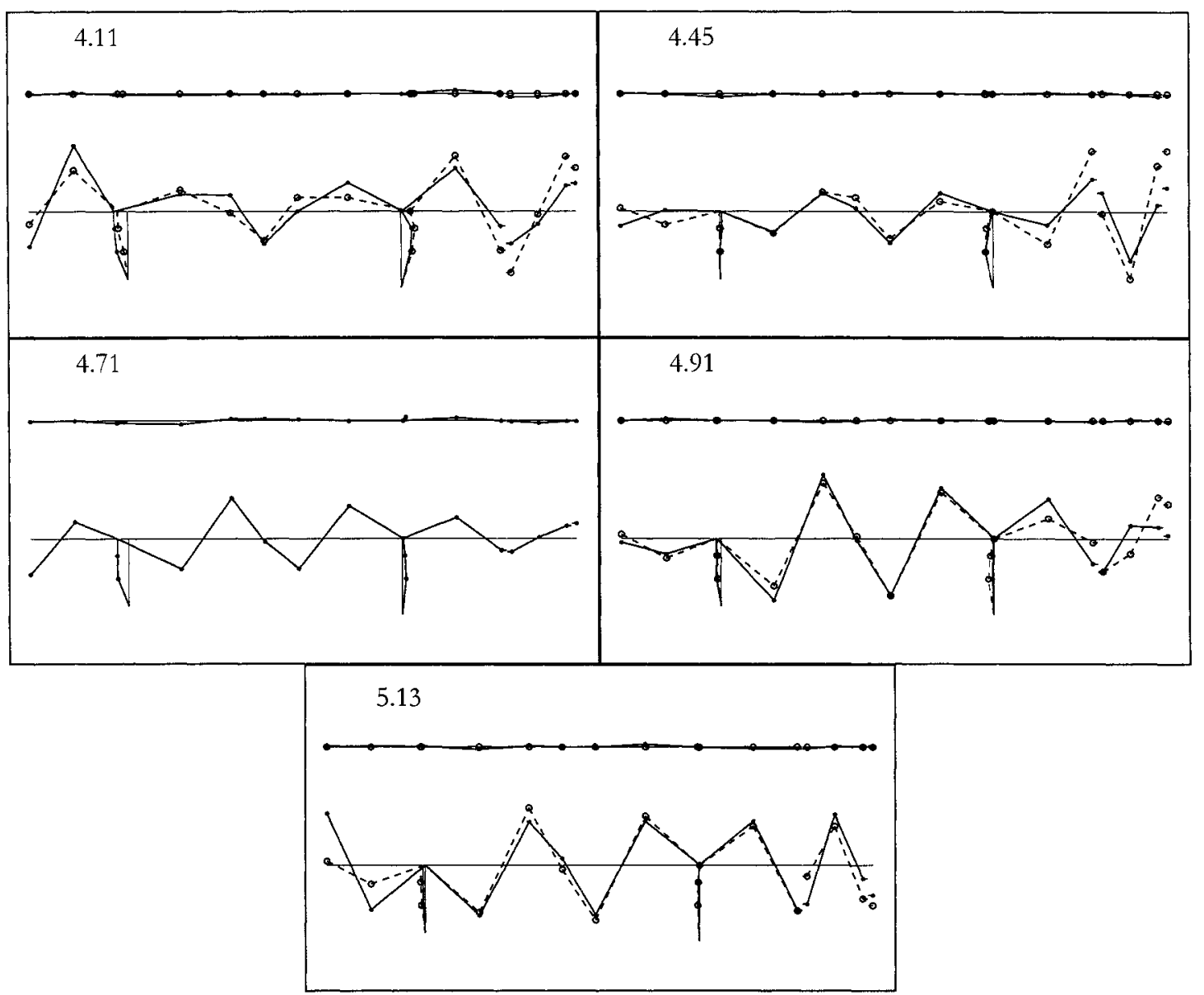

Figure 2-7C: Mode shapes. Labels indicate mean extracted frequency. Markers indicate sensor locations. Extracted modes are shown in full line, full matkers; theoretical shown in dotted line, open markers. 


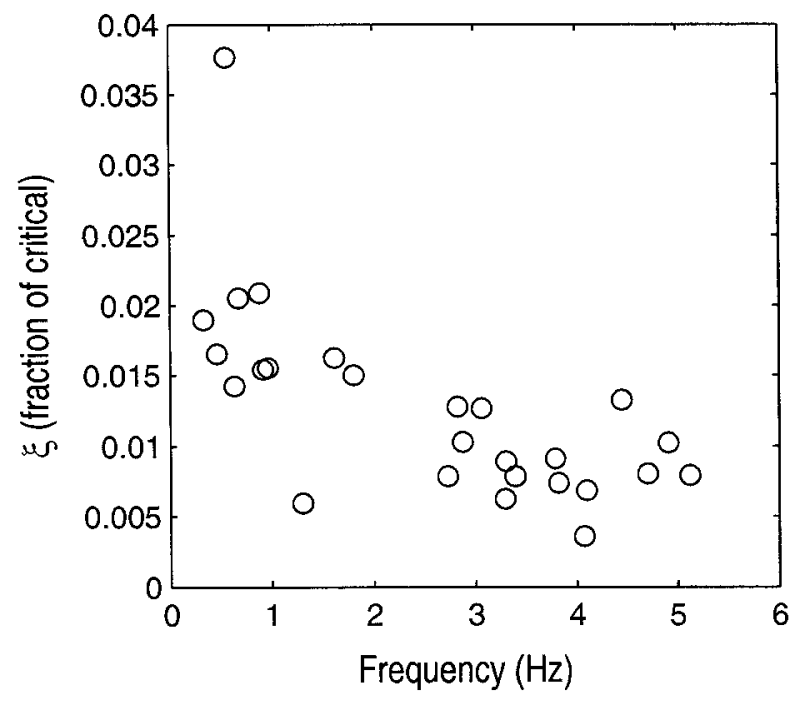

Figute 2-8: Modal damping ratio versus modal frequency 


\section{Chapter 3. Variability of dynamic properties from field data and implications for health monitoring}

\subsection{Introduction}

Maintaining vital civil infrastructure lifelines, such as bridges, in good operating condition is one of the priorities in civil engineering. To determine maintenance and repair needs and requirements for these structures, some form of structural evaluation is normally carried out either on a regular scheduled basis or following the occurrence of extreme loading events, which may arise from natural or man-made disasters such as earthquakes, landslides, hurricanes, flooding and collisions. Such evaluations are meant to determine the location, extent and severity of damage, which may be caused either by gradual deterioration and fatigue or by the exceedance of design loadings and displacements. Until now, structural evaluation has been largely based on visual inspections, sometimes aided by localized measurement methods such as acoustic, ultrasonic and magnetic field techniques. These localized techniques have important limitations such as inaccessibility to some parts of the structure, inability to detect hidden internal damage, the need of a priori knowledge of damage location, and that they can only be carried out at periodic intervals. Consequently, the ability of the visual inspections to detect early signs of deterioration, which is desirable to devise cost effective counter-measures, is limited. The worldwide problem of rapidly rising maintenance costs due to aging urban infrastructure, currently around $\$ 6$ billion annually in Canada alone (CPWA, 2005), calls for the development of better structural evaluation tools that overcome the limitations of the current methods to help curb the rising costs.

The new technology of vibration-based structural health monitoring (VBSHM), originally developed in the automotive and aerospace industries, could be the answer to this 
problem. The basic principle of VBSHM is that structural vibration characteristics are functions of the structure's physical properties (stiffness, mass, damping). Therefore, by measuring changes in the vibration characteristics it is possible in theory to determine changes in the physical conditions of the structure arising from damage or deterioration. VBSHM has seen many developments in recent years as evidenced in recent literature reviews and conference proceedings (for instance: Sohn et al. 2003, Wu and Abe 2003) and numerous monitoring systems have been developed and installed in the field to collect information on the material properties and structural behaviour of civil engineering structures and systems, such as buildings and bridges (e.g. Abe et al. 2000, Kim et al. 2000, Wong et al. 2000, Katsuchi et al. 2003, Koh et al. 2003, Peeters et al. 2003). The growing popularity of VBSHM is owing not only to it attractiveness as a promising alternative to visual inspection-based evaluation but also to the parallel development of information technologies which have made it possible to collect, transmit and process the large volumes of data typically involved in monitoring projects within reasonable time frames.

One of the key advances so far in VBSHM has been the development of powerful numerical techniques for the system identification analysis of vibration response monitoring data (Peeters and De Roeck 1999, Yang et al. 2003). With these techniques, the dynamic properties (frequencies, mode shapes and damping ratios) of an instrumented structure may be obtained from output-only vibration measurement data, where only the outputs or vibration responses of the structure are measured. As opposed to traditional input-output testing methods, where carefully controlled loadings or inputs are applied and measured, in the output-only case random ambient loadings such as wind and traffic are relied upon as the sources of excitation. This is attractive for health monitoring of civil structures because it is often too expensive or simply not feasible to induce vibration artificially in large size civil 
structures by controlled force excitations. Even in the cases of relatively small structures where it might be feasible to induce vibrations artificially, output-only monitoring can be more attractive because it can be implemented at a much lower cost than the traditional input-output modal testing without service interruptions. Additionally, output-only measurements can be implemented in a continuous fashion for long-term monitoring, which is not the case for input-output testing. The trade-off is that ambient vibration response data typically contains significantly higher noise-to-signal ratios than those obtained in traditional input-output settings, where the loading can be carefully controlled and measured. In the output only case, the ambient loading spectrum varies with time and is typically not flat, which may result in only some vibration modes being excited at any given time. The lack of excitation information and control coupled with the high level of uncertainty in the operating environment of civil structures make it highly challenging to use the ambient vibration data to detect structural damage or deterioration in civil engineering applications.

Several key areas of research requiring important advances before realistic practical applications of vibration-based damage detection can be implemented have been identified (Doebling et al. 1996, Humar et al. 2003). Concerted efforts involving university and industry partners, such as the EU-funded Structural Assessment, Monitoring and Control (SAMCO) initiative, are currently underway to harmonize and speed up the development of vibration based health monitoring. One of the major difficulties to be overcome stems from the fact that changes in the measured vibration characteristics of the monitored structures do not arise exclusively from damage but also from a variety of sources including the environmental fluctuations that affect the behaviour of the structural system and also from measurement, modeling and computational uncertainties. Therefore it is difficult to distinguish which changes are due to damage and which are not. This has previously been recognized in the 
literature (Farrar et al. 1997, Alampalli 1998, Sohn et al. 1998, Rohrmann et al. 2000) but still remains an outstanding problem. If the normal variability of the measured vibration characteristics can be fully understood and quantified, then it will be possible to determine if observed changes in the vibration signatures are indeed caused by structural damage or merely attributable to the variability.

The comprehensive monitoring project of the Confederation Bridge, in eastern Canada, represents a unique and ideal opportunity to advance our knowledge in VBSHM thanks to the long-term availability of continuous monitoring information covering not only the dynamic responses of the structure but also additional variables such as concrete temperatures, material properties, wind speeds, etc., which can help characterize and understand the structural behaviour perhaps better than ever before. In addition to this, thanks to the location of the bridge crossing the marine waters of the Northumberland Strait, the project covers an unprecedented range of environmental scenarios from hot calm summer days to extremely cold and windy winter days with significant seasonal ice floes. Owing to the bridge's location, the average temperatures of the concrete in the bridge girders can range from around $-20^{\circ} \mathrm{C}$ to $+25^{\circ} \mathrm{C}$ in a given year while winds frequently exceed 100 $\mathrm{km} / \mathrm{h}$.

In this chapter, a study of the variability of the dynamic properties of the Confederation Bridge extracted by a modern subspace method from output-only vibration monitoring data is presented. An attempt has been made to separate and quantify the variability arising from (1) the measurement, modeling and computational uncertainties and that caused by (2) the environmental and loading fluctuations.

This chapter is divided into four sections; the first section is an attempt to identify all the different sources of uncertainty in the monitoring data that could potentially cause 
variability in the extracted dynamic properties. In the second section, the variability arising from the measurement, modeling and computational uncertainties is determined. This variability referred to as the "baseline" variability. The baseline variability study is based on the analysis of 10 monitoring datasets collected under similar environmental and loading scenarios over a relatively short time frame (2-days). The third section examines the variability under fluctuating environmental and loading conditions by considering 22 datasets collected over a 6 -month period. The fourth and final section compares the variability results of Sections 2 and 3 against the sensitivity of the bridge's modal parameters to simulated damage scenarios in order to evaluate the feasibility of using the monitoring data for damage detection.

\subsubsection{Continuous monitoring system}

A comprehensive long-term monitoring system on the Confederation Bridge has been in operation since the bridge opening in 1997 to collect data and information about its behaviour and performance. The monitoring system records both environmental and bridge response data including vibration responses, concrete temperatures via numerous embedded thermocouples, short and long term deformations, ice cover conditions and interactions with the piers, and weather data. The dynamic monitoring system is dedicated to the measurement of the vibration responses of the bridge caused by significant sources of dynamic excitations, including wind, heavy traffic, ice loads and earthquakes. The vibration instrumentation comprises a total of 76 accelerometers distributed around a typical structural frame module, as shown in Figure 3-1. Vibration responses of the bridge girders are measured in the vertical and lateral directions as shown in Figure 3-1. This setup facilitates the recovery of vertical bending, lateral bending and torsional vibration modes of the bridge 
superstructure. The response behaviour observed in the instrumented segment of the bridge is considered representative of the behaviour of the main-spans portion of the structure.

\subsubsection{System identification}

Subspace methods such as eigensystem realization algorithm (ERA), covariance block Hankel matrix (CBHM), canonical variate analysis (CVA), balance realization (BR) \& stochastic subspace identification (SSI) are amongst the most advanced methods presently available for the modal analysis of vibration data, especially in the output-only case, as corroborated by the results of a recent comparative investigation by Peeters \& Ventura (2003). In their study, seven research teams from around the world were invited to independently analyze the same set of measured vibration data using their own system identification methods of choice. The comparison of the different results showed that the subspace techniques were generally more effective and consistent in extracting vibration modes from the field measured data than the other five techniques used by the different participants, given that the analysts used appropriate values for the analysis parameters required by the subspace method. It is worth noting that this technique performs very well at extracting closely spaced vibration modes.

In the present investigation, a correlation driven variant of the SSI method, as presented in Section 2.5.2, together with the stabilisation diagram technique, is used for the modal analysis of the vibration data of Confederation Bridge. The SSI method has been implemented by the author in collaboration with S.L. Desjardins as part of a modular software tool in Matlab incorporating data processing, analysis and visualization modules, which together serve as a platform for the health monitoring of Confederation Bridge (Desjardins et al. 2006) 


\subsection{Potential sources of variability}

There are numerous variables that may potentially affect the values of structural dynamic properties extracted from vibration monitoring data collected from civil structures in the field. In the present section, an effort has been made to recognize and classify as many of the potentially significant sources of variability. Obviously, some sources may be more significant than others, and their relative importance will be specific to each monitoring project. Although variability is a critical problem for damage detection methods that rely on the detection of changes in the dynamic properties, surprisingly little is presently published and understood about it. One thing is sure, if damage detection is to be implemented reliably in the field, all significant sources of variability must be understood and accounted for.

The sources of variability can be grouped into two major categories

I. Modeling, measurement and computational uncertainties

II. Environmental and loading fluctuations

The difference is that sources of variability of the first category do not change the actual values of dynamic properties of the system but rather affect only the observed or extracted values. On the other hand, sources in the second category may actually change the values of dynamic properties of the system as well as potentially affecting the observed values. For simplicity, sources in Category I are herein after referred to as "numerical" sources, while those in Category II are called "physical" sources.

To clarify the discussion, it is assumed here that the system identification technique employed is the correlation driven SSI method presented earlier. However, the discussion is still generally applicable to any method.

The numerical sources of variability (Category I) can be divided into the following subcategories 
(1) Deviations from modeling assumptions. The data collected from real structures is likely to deviate by some amount from the various assumptions used in deriving the system model that serves as a basis for the system identification. In particular, the vibration data may deviate from the linearity assumption of the structural model and also from the stationarity assumptions, which are implicit in many of the existing system identification methods. Little is known of how this may affect the values of dynamic properties extracted from the data. However, in regards to the effect of deviations from stationarity on the stochastic subspace identification technique, Benveniste \& Fuchs (1985) show that this technique is robust against non-stationary inputs. Deviation from other assumptions such as the assumption of whiteness of the stochastic input terms is also most likely, especially in the case of vibration under operational excitation forces, because the spectrums of operational loadings such as wind and traffic are not necessarily flat. In this respect, Bogunović Jakobsen (1995) proposed a filtering technique to correct the responses for the non-whiteness of the input if the shape of the loading spectrum is known.

(2) Measurement uncertainties. These may arise from a variety of factors anywhere along the measurement chain, which typically involves the sensors, wiring, power supply, signal conditioning, filtering hardware and data loggers. Measurement uncertainties can usually be minimized through careful instrumentation and data acquisition practices.

Nonetheless, some amount of measurement error tends to be present in any measured data and it can often become important, especially at low signal levels. Measurement errors may arise from the following

a) The sensors

b) Electrical noise or interferences associated to the wiring and electronics 
c) Filtering distortions

d) Sampling noise introduced by the analogue to digital (A/D) discretization of the data. Analogue to digital conversion of the data is limited to the finite resolution of the converter, which is $\Delta V=\frac{V_{\text {range }}}{2^{n}}$, where $V_{\text {rauge }}$ is the preset voltage range used for the measurement channel, and $n$ is the number of bits of the converter. This implies that sampling noise can become important if the signal level only occupies a small portion of the preset voltage range.

e) Noise introduced by the finite precision of data storage

(3) Computational uncertainties, such as those arising from

a) The use of finite datasets to compute estimates of the output correlation matrices. As a result, datasets of different durations will result in slightly different estimates.

b) Noise and distortions in the data introduced by offline data processing operations such as filtering and re-sampling.

c) The analyst and his experience. For complex problems, such as practical system identification, different analysts may often obtain significantly different solutions. The results obtained by Peeters \& Ventura (2003) highlight the significance of this factor. The reason is that judgment is required in some way or the other by most system identification methods. Developing reliable intelligent algorithms that eliminate this requirement remains a major challenge in system identification. As an example, in the stochastic subspace method, the judgement of the analyst plays a role in the following

i) Selection of the portion of the correlations to be used for system identification (i.e. selection of the " $\imath$ " parameter). 
ii) Selection of reference sensors for computation of data correlations. This represents an important subjective step in the identification process requiring the judgment and experience of the analyst. Different selections of reference sensors may lead to slightly different identification results, including the omission or poor identification of important vibration modes. The criteria in selecting the reference outputs are analogous to the selection of input locations in traditional input-output modal testing.

iii) Selection of system eigenvalues. To work around the limitation of the unknown order of the system, it is common practice to identify solution sets of different model orders and display them in stabilisation diagrams. This allows the analyst to distinguish stable solution trends and extract stable system eigenvalues from the diagram. The stabilisation diagram is a powerful tool in the sense that it shows stabilized trends only for the physically meaningful solutions. However, the exact order of the system solution, at which the eigenvalues should be taken from the diagram, is not directly evident and the analyst must usually rely upon his judgement to select an approximate order. Obviously this can lead to variability in the extracted results because there is inevitably some change in the solutions of different order.

d) The system identification method. Different system identification methods tend to yield different solutions for the analysis of the same dataset (Ndambi et al. 2000, Peeters and Ventura 2003).

e) Noise introduced by machine round-off during computations. 
The Category II or "physical" sources of variability can de divided into the following subcategories

(1) Environmental fluctuations. Changes in temperature and temperature differentials have been recognized to have important effects on the identified eigenfrequencies (Farrar et al. 1997, Sohn et al. 1999, Rohrmann et al. 2000, Peeters et al. 2001, Ko et al. 2003). This may result partly from temperature-induced changes in the material properties of the different structural components. In particular, the elastic modulus of concrete is known to increase with decreasing temperatures, as observed in the studies by Lee et al. $(1988 \mathrm{a}, \mathrm{b})$ in the range of $20^{\circ} \mathrm{C}$ to $-70^{\circ} \mathrm{C}$. The effects of temperature may be particularly important for bridges exposed to large seasonal fluctuations, such as the Confederation Bridge. Alampalli (1998) suggests that changes in the boundary conditions due to freezing of the supports may also have a significant effect. Sohn et al. (1999) suggests that changes in mass due to moisture absorption and retention may have a significant effect on the eigenfrequencies. Other environmental variables such as presence and thickness of ice around the piers (for bridges built over ice-covered water), which might restrict pier motion, and other less frequent conditions such as ice-accretion, may all play some role in affecting the dynamic behaviour of the structural system.

(2) Differences in loading scenarios. Potentially, data collected for the same structure under similar environmental conditions but different loading scenarios may lead to different identification results. The loading scenarios may vary according to:

a) The type of excitation. Artificial excitations can be applied to the structure by means of impact devices such as impact hammers, electro-dynamic shakers or sudden unloading (pull tests). Otherwise, ambient loadings can be relied 
upon to induce the structural vibrations. The ambient loadings may involve different combinations of traffic, wind, ice actions, waves, currents and earthquakes. Ndambi et al. (2000) used different artificial excitations in laboratory tests on a reinforced concrete beam and found some changes in its identified dynamic properties attributable to the changes in the type of excitation. The results of Peeters \& Ventura (2003), who considered both artificial and ambient excitations for a bridge, also found some changes between the modal parameters extracted from datasets of different excitation type.

b) The amplitude of the excitation. The amplitude of the excitation, which is normally also reflected in the amplitude of the response, has been observed to influence the identified parameters. Ndambi et al. (2000) performed modal analyses at different amplitudes of excitation using swept-sine excitation. They found that the extracted natural frequencies of the tested specimen decreased with increasing excitation amplitude. Modal damping ratios were generally observed to increase with the excitation amplitude. These observations are corroborated for a large scale field structure by the results of Abe \& Siringoringo (2003), who identified dynamic parameters for the longspan Hakucho suspension bridge from both ambient and forced vibration data.

c) The changes in the mass of the system from the presence of heavy-traffic (Farrar et al. 1997).

d) The extent of vehicle-bridge coupling. During passage of a heavy vehicle over a bridge span, vehicle and bridge may behave as a coupled dynamic system, as 
observed by Cantieni (1993). Therefore, when a heavy vehicle passes over a bridge span, bridge response frequencies may shift temporarily to the frequencies of the coupled system. The shift is more significant when the natural frequencies of the bridge and vehicle are similar but it also depends on vehicle speed and pavement unevenness. The result is that the measured bridge vibrations can have a non-stationary behaviour with coupling occurring when the vehicle is passing over a given span followed by decoupling as the vehicle leaves the span. The frequency shifts produced by this behaviour can be more significant than those produced by the additional vehicle mass alone.

e) The amount of aerodynamic damping and stiffness introduced by wind which may change the effective stiffness and damping of the system. This effect may be more important for relatively flexible structures such as suspension bridges.

\subsection{Baseline variability}

The baseline variability is defined here as the variability in the identified system parameters attributable to the Category I (or "numerical") sources of variability, i.e. the modeling, measurement and computational uncertainties described in the previous section. The baseline variability would be the only type of variability if datasets were collected under identical environmental and loading scenarios, given that no change occurs in the structural condition of the system. Ideally, under such conditions, there should be no change in the extracted dynamic properties. Therefore, the baseline variability may be considered a measure of the quality of the monitoring data, the modeling assumptions and the analysis procedures. Naturally, before studying the variability under more general circumstances, it is 
desirable first to quantify the baseline variability, since it is expected to always be a component of the total variability.

It is possible to approximately quantify the baseline variability by considering datasets collected during a short period of time at similar times of the day and under similar loading and environmental scenarios, assuming that the small differences in the scenarios have negligible effects. This has been the approach taken in the present study, where ten datasets of vibration responses of the Confederation Bridge collected over a two-day period under similar conditions have been analyzed via the SSI method to estimate the baseline variability of the extracted modal vibration parameters.

\subsubsection{Data}

The ten datasets of this study were recorded around mid-day on March 13 and 142003. Each recorded dataset is of ten minute duration and comprises the simultaneously recorded bridge acceleration responses measured by 15 accelerometers corresponding to monitoring locations 5 through 9 of Figure 3-1. The average temperatures of the concrete, measured by thermocouples embedded in the bridge girder, and the average wind speeds at the time of collection of each dataset are presented in Figure 3-2, showing that the environmental conditions of the datasets considered for the study vary only within a relatively narrow range. The average temperature of the concrete, measured by thermocouples embedded in the bridge girder, ranges between -2.7 to $-1.4{ }^{\circ} \mathrm{C}$, while the 10 -min average wind speed is relatively steady at $11 \pm 3.8 \mathrm{~m} / \mathrm{s}$ at the times of collection of the datasets. The respective slight fluctuations in the temperature and wind speed conditions are considered negligible when compared to the corresponding yearly ranges that can exceed $45^{\circ} \mathrm{C}$ and $30 \mathrm{~m} / \mathrm{s}$. Time history plots of acceleration responses from sensor location 7 and the corresponding power 
spectral density estimates (PSD) showing the frequency content of the signals are shown in Figure 3-3 and Figure 3-4 respectively. As may be observed from the plots, the patterns of the response time histories and the frequency content of the responses belonging to the different datasets have comparable characteristics, which reflects the similarity in the prevailing loading conditions at the times of data acquisition. Bridge response recordings of a relatively long duration are used in this study in order to improve the quality of the data correlation estimates, which form the basis of the SSI system identification technique employed in the extraction of the modal vibration properties. An example of a typical data correlation estimated from the measured responses is shown in Figure 3-5.

The dynamic behaviour of the Confederation Bridge has been discussed in Chapter 2, as well as other recent investigations (Zhang 2001; Naumoski et al. 2002, 2004; Lau et al. 2004). In the work presented in Chapter 2, twenty-five global vibration modes of the Confederation Bridge were identified, with natural frequencies ranging from 0.34 to $5.13 \mathrm{~Hz}$. The present study concentrates on the variability of the four following vibration modes: 0.48 $\mathrm{Hz}, 1.64 \mathrm{~Hz}, 1.82 \mathrm{~Hz}$ and $2.77 \mathrm{~Hz}$, of which the corresponding mode shapes over the full instrumented bridge segment are shown in Figure 3-6. These four modes have been selected for this investigation because they can be extracted from most monitoring datasets and because they do not have closely spaced counterparts. Other modes have been excluded, either because they are difficult to extract consistently from different datasets, or because they have closely spaced counterparts with very similar mode shapes within the bridge segment being considered (monitoring locations 5 to 9), which may make it difficult to differentiate between them. 


\subsubsection{Data processing}

To prepare the monitoring data for system identification analysis, the following processing operations are performed on the accelerometer time-history signals:

(1) Baseline offset adjustment.

(2) Purging of duplicate data records.

(3) Patching small data gaps. Where data samples are found to be missing, a cubic polynomial interpolation algorithm patches small data gaps by taking a set number of samples before and after the missing samples in the interpolation.

(4) Low-pass filtering. A Chebyshev Type II filter of order 13 with a stop-band edge frequency of $9 \mathrm{~Hz}$ is used. The filter type and order are designed for minimal data distortion. The filter fulfills two purposes: noise reduction in the band of interest $(0-3$ $\mathrm{Hz}$ ) and anti-aliasing in the subsequent down-sampling operation. With the cut-off frequency at $9 \mathrm{~Hz}$, unwanted frequency components associated to localized vibrations of the girder occurring mainly in the $10-15 \mathrm{~Hz}$ band are removed. These localized vibrations have been reported by Zhang (2001), Naumoski et al. (2002) and modeled in detail by Cheung et al. ${ }^{2}$. Data is filtered in the forward and backward directions to eliminate phase distortion.

(5) Down-sampling of the data to one-third of the original sampling rate. This substantially reduces the size of the data for economy in subsequent computations without any significant loss of resolution.

\footnotetext{
${ }^{2}$ Cheung MS, Lau DT and Li WC. Local vibration of girder box on Confederation Bridge, Internal report, Ottawa-Carleton Bridge Research Institute, Carleton University.
} 
All the above processing tasks are carried out using the structural monitoring application platform developed by Desjardins et al. (2006).

\subsubsection{System identification}

The processed datasets are analyzed using the SSI method presented earlier with the following analysis parameters:

(1) Maximum state-space model order $\left(n_{\max }\right): 150$. This number corresponds to 75 structural vibration modes and is expected to exceed the true model order significantly. Although this parameter does not have a direct repercussion on the analysis results, selecting a relatively large number helps visualize stabilised trends in the stabilisation diagram.

(2) Reference outputs. The three accelerometers at location $7(r=3)$ are selected to serve as references for system identification. Location 7 is away from the modal nodes of the modes of interest and thus carries the all the desired modal information with good signal-to-noise ratios, a fact that is corroborated from observation of the corresponding mode shapes and PSDs.

(3) Number of time lags of the data correlation matrices ( $i$ parameter): 200 . Two criteria are used in selecting this important system identification parameter. First, it must satisfy the mathematical requirement of $r \times i>n_{\max }$. Second, from the authors' experience, the length of the data correlations should ensure that the modal information of the lowest modes is conveyed accurately by covering at least one or two complete vibration cycles of the lowest mode to be identified. A value of $i=200$ (with $d t=0.024 \mathrm{~s}$ ) results in data correlations that include more than two full cycles of the lowest vibration mode $(0.48 \mathrm{~Hz})$, which is considered satisfactory. 


\subsubsection{Results}

Table 3-1 and Figure 3-7 summarize the results of the baseline variability study for the modal vibration frequencies, damping ratios and mode-shapes identified from the ten different vibration datasets. In Figure 3-7, frequency and damping values are normalized by the mean values. Mode shape variability is assessed via the Modal Assurance Criterion (MAC), which is a common mode comparison technique (Maia et al. 1997). The MAC is essentially a normalized projection of one mode vector onto the other, with a value of 1 indicating perfect correlation and a value of 0 indicating no correlation. The values of MAC shown in Figure 3-7 (and Figure 3-8) are computed as follows

$$
M A C_{i}=\frac{\left|\boldsymbol{\psi}_{i}^{n^{T}} \overline{\boldsymbol{\Psi}}\right|^{2}}{\left(\boldsymbol{\psi}_{i}^{n^{T}} \boldsymbol{\psi}_{i}^{n}\right)\left(\overline{\boldsymbol{\Psi}}^{T} \overline{\boldsymbol{\Psi}}\right)}
$$

where $\psi_{i}^{n}=\frac{\left|\psi_{i}\right|}{\left|\psi_{i}^{r e f}\right|}$ is the vector of normalized mode shape amplitudes extracted from the $i-$ th dataset, the symbol ${ }^{T}$ indicates transpose, and $\bar{\psi}$ is the average of the $M$ vectors of normalized mode shape amplitudes extracted from the $M$ different datasets being considered. Normalization of the mode shape amplitudes is carried out by dividing the amplitude of each modal coefficient by the amplitude of an arbitrary reference coefficient $\left(\left|\Psi_{i}^{r e f}\right|\right)$ with a relatively large amplitude. The same reference node is used for each set of modes.

Since it is difficult to determine the true order of the system, the SSI solutions obtained at two different model orders are shown, a lower order solution, of model order around 40, and a higher order solution of model order typically around 70 . As may be observed from the plots of Figure 3-7, the baseline variability of the eigenfrequencies for the four modes considered is typically within the $\pm 1.0 \%$ band. The corresponding average 
standard deviation is $0.5 \%$ from the mean, as found in Table 3-1. The identified mode shapes are also relatively consistent, with MAC values that tend to be above 0.995 . However, as may be observed from Figure 3-7, some mode shapes seem to exhibit slightly more variability than others. For example, the MAC values for the $2.77 \mathrm{~Hz}$ mode are more scattered than those of the $0.47 \mathrm{~Hz}$ mode. In addition, some "outlier" mode shape identifications occur, such as the one observed in the MAC plot of the $1.82 \mathrm{~Hz}$ mode.

While the MAC values in Figure 3-7 are computed between extracted mode-shape amplitudes and the corresponding average extracted mode-shape amplitudes, the average MAC values of Table 3-1 compare the complex modal vectors identified from different datasets against each other as follows

$$
\overline{M A C}=\frac{\sum_{i=1}^{M} \sum_{j=i+1}^{M} M A C\left(\boldsymbol{\psi}_{i}, \boldsymbol{\psi}_{j}\right)}{\sum_{i=1}^{M} \sum_{j=i+1}^{M} j}
$$

where $M A C\left(\boldsymbol{\psi}_{i}, \boldsymbol{\psi}_{j}\right)$ is computed using the complex modal vectors according to Eq. (2-13). It should be noted that this expression tends to yield lower values of average MAC than if an average of the values of Figure 3-7 were taken. As an example, the overall average of MAC computed for the $0.48 \mathrm{~Hz}$ mode with the above expression is 0.988 , whereas the average of MAC values of Figure 3-7 for this mode is 0.998.

In contrast to the relatively good consistency observed for the extracted modal frequencies and mode shapes, a much larger variability is obtained for the damping ratios, with discrepancies between extracted values that can sometimes be close to a factor of 2 and with an average standard deviation of $35 \%$ of the mean. The higher variability of the extracted damping ratios is not surprising given the high complexity and uncertainty inherent 
to damping phenomena, especially in large scale concrete structures with complex boundary conditions such as the Confederation Bridge.

As a general observation, the discrepancy between the modal parameters of "lower" and "higher" model order is not that significant as compared to the overall variability, which to a certain degree gives confidence in the system identification solutions.

The variability of mode-shapes is examined further in Figures 3-9 and 3-11, which show the variability of individual modal amplitudes at the different sensor locations. From Figure 3-9 it can be observed that there is more variability at some sensor locations than others. This localized variability may result from larger noise-to-signal ratios at those particular sensor locations (from Category I sources of uncertainty) or from a larger sensitivity at these particular locations to small changes in the system. The results of mode shape variability, including those shown in Figure 3-11 are discussed further in the following section.

\subsection{Variability under environmental \& loading fluctuations}

In contrast to mechanical and aerospace structures, which are usually tested in carefully controlled laboratory conditions, civil engineering structures are normally subjected to a wide range of uncontrollable environmental and loading scenario combinations. To perform reliable vibration based structural health monitoring in civil engineering, it is necessary to investigate and understand how these fluctuations affect the data acquired in the field. The Confederation Bridge monitoring project offers an ideal setting to do this because of the wide range of environmental and loading conditions that the bridge is subjected to as a result of its location across the Northumberland Strait and because of the availability of comprehensive monitoring data covering many of the potentially relevant variables. 


\subsubsection{Data}

Twenty-two datasets collected over a six month period, spanning from mid-summer to mid-winter, are selected from the monitoring database in an attempt to cover the widest possible range of environmental and loading fluctuations. For the selected time period, average measured temperature of the bridge girder concrete varies between $-20^{\circ} \mathrm{C}$ and $+25^{\circ} \mathrm{C}$. As discussed in Section 3.2, previous studies coincide in observing that temperature plays an important role in affecting the extracted modal parameters. Hence, the main criterion for the selection of datasets for this study is based on temperature. The criterion is to have datasets at equally spaced temperature increments to avoid bias towards any particular temperature range. One dataset per each two-degree-wide bin of average concrete temperature is selected from the monitoring database, resulting in the twenty-two datasets. Apart from the temperature criterion, selection of the datasets is essentially random, except that datasets with very low amplitudes are discarded because of high noise-to-signal ratios. Time history plots from the 22 datasets are shown in Figure 3-12, while power spectral density estimates showing the corresponding frequency content are shown in Figure 3-13. In comparison to the baseline data plots of Figure 3-3 and Figure 3-4, both the time and frequency plots show that the patterns of the bridge response traces under the different loading scenarios can vary quite significantly. In addition to the obvious differences in amplitude and frequency characteristics, a marked difference is observed in the stationarity of the signals, some showing a more stationary behaviour than others. As an example, the response record of $06 / 10 / 200310: 35$ shows significant non-stationary characteristics over the duration of the ten minute recording while that of $13 / 11 / 200316: 10$ seems to better approximate the definitions of a stationary random process. 
In this study, average temperatures of the bridge concrete are obtained from a total of 156 thermocouples embedded in the girder concrete at three different girder cross sections located in the vicinity of monitoring locations 9,10 and 11 respectively. The typical cross section thermocouple layout is shown in Figure 3-14. For more details of the temperature monitoring aspects of the Confederation Bridge the reader may refer to Li et al. (2003). Average concrete temperatures for the six-month time period under consideration along with wind speed data recorded at the bridge are shown in Figure 3-15. From the wind speed data it is observed that the bridge is quite often subjected to high winds, with 10 -min average speeds often exceeding $20 \mathrm{~m} / \mathrm{s}$. The wind speeds for the 22 events selected for the present study range from $2.8 \mathrm{~m} / \mathrm{s}$ to $22 \mathrm{~m} / \mathrm{s}$.

\subsubsection{Data processing and system identification}

The same data processing techniques and system identification parameters as in the baseline variability study presented earlier are used.

\subsubsection{Results}

Figure 3-8 and Table 3-2 summarize the variability of the modal parameters extracted from the 22 different datasets spanning the six-month period considered here under combined environmental and loading fluctuations. In comparison to the baseline variability results of Figure 3-7 and Table 3-1, it is observed that there are higher variations in the identified eigenfrequencies, with an increase in the average standard deviations of the modal frequencies by a factor of around 2 , from $0.5 \%$ to around $1 \%$ of the mean. Hence, about half of the variability in the modal frequencies is attributable to the physical - Category II sources of variability while the other half can be attributed to numerical sources of uncertainty. 
On the other hand, the standard deviations of the damping ratios remain essentially unchanged as compared to the baseline variability, at about $30 \%$ of the mean, which suggests that the variability of extracted damping ratios is not increased by the loading and environmental fluctuations. In fact, after exploring several potential relations between damping and variables such as temperature, wind speed and vibration amplitude, no significant relation was observed, except for the $1.6 \mathrm{~Hz}$ mode, for which the damping seems to exhibit a relation to the vibration amplitude, as shown in Figure 3-17. With this exception aside, the lack of correlations for the extracted damping ratios suggests that the variability in the damping ratios stems mostly from the inherent complexity of energy dissipation mechanisms which are difficult to represent accurately in the viscously damped system model, unlike the structural stiffness and mass parameters.

In regards to the mode shapes, comparison of Figures 3-7 and 3-8 clearly indicates a decrease in the overall consistency of the mode shapes extracted under random environmental fluctuations with respect to those extracted under similar conditions, a fact corroborated by the average MAC values of Table 3-2 as compared to those of the baseline variability study in Table 3-1. The corresponding increase in the variations of the modal amplitudes of individual mode shape coefficients can be observed in the plots of Figure 3-10 as compared to those of Figure 3-9. Again, the variability appears to not be uniform, i.e. it is larger at some modal coefficients than others, which is undesirable for damage detection.

The variability of the mode shapes is illustrated further in Figure 3-11, where the average normalized mode shape amplitudes are shown with error bars indicating the standard 
deviations from the mean. ${ }^{3}$ Since this figure shows the standard deviations superimposed on the actual mode shapes it may tend to give a better physical insight into the mode shape variability than the previous figures. Here, only vertical or lateral results are shown depending on the type of mode. From Figure 3-11 it may be observed that the level of variability is actually not as large as it would appear to be from the previous two figures. The standard deviations corresponding to Figure 3-11 are given in Table 3-4. Understandably, the standard deviation percentages are generally smaller for those locations with larger modal amplitudes, which tend to have better signal-to-noise ratios. For those locations, the baseline standard deviations of the modal amplitudes can be as low as $0.5 \%$ from the mean but in general they tend to be roughly between $1 \%$ and $6 \%$. A weighted average standard deviation can be computed as follows

$$
\bar{\sigma}(\%)=\frac{\sum_{i=1}^{\text {ndof }} \sigma_{i}(\%) \cdot\left|\psi_{i}^{n}\right|}{\sum_{i=1}^{n d o f}\left|\psi_{i}^{n}\right|}
$$

where the normalized amplitudes $\left|\psi_{i}^{n}\right|$ are used as weights, $\sigma_{i}(\%)$ represents the standard deviation as a percentage of the mean of the i-th degree of freedom (DOF), and ndof represents the number of DOFs. Equation (3-3) yields average standard deviations of $3.9 \%$

\footnotetext{
${ }^{3}$ For the calculation of the standard deviations of Figure 3-11 and Table 3-4, the mode shapes identified from the different datasets, are normalized by the root-mean-square of their amplitude, which is defined here as $m_{a}=\frac{1}{n d o f} \sqrt{\sum_{i=1}^{n \text { dof }}\left|\phi_{i}\right|^{2}}$. This normalization is preferred over the usual normalization procedure using the amplitude of a single modal coefficient (e.g. the one with maximum amplitude) because it is less biased towards a particular coefficient. The traditional procedure would result in zero variance for the reference modal coefficient, which is obviously not realistic.
} 
for the baseline datasets and $5.5 \%$ for the general datasets. In light of these results, the amount of variability traditionally assumed in damage detection simulation studies for the mode shapes, which is usually $5 \%$ seems to be reasonable. Nonetheless, the fact that the real variability is non-uniform might be an important factor which is not considered in most simulation studies. In addition to the above, the results of average standard deviations corroborate the observation that the random environmental fluctuations lead to increased mode shape variability as compared to the baseline variability.

The relation between the modal frequencies and the average temperature of the concrete structural members of the Confederation Bridge for the four vibration modes considered in this study is shown in Figure 3-16. The plots reveal an inverse trend of decreasing vibration frequency with increasing concrete temperatures similar to that reported by other researchers (Peeters et al. 2001, Rohrmann et al. 2000). This explains the decreasing trend observed in the eigenfrequency plots of Figure 3-8 resulting from the ordering of the datasets according to increasing concrete temperature. The observed trend, which seems to be close to a linear trend, is more clearly defined for the $1.8 \mathrm{~Hz}$ and $2.7 \mathrm{~Hz}$ modes, with less dispersion than the $0.47 \mathrm{~Hz}$ and $1.6 \mathrm{~Hz}$ modes. The observations are significant as this is one of the few cases that the temperature effect on the eigenfrequencies is determined and reported for a long-span prestressed concrete bridge over a wide temperature range. Table 3-3 shows the results of linear regressions of frequency vs. average concrete temperature. Correlation coefficients $\left(r^{2}\right)$ for the linear fits corresponding to the 1.8 and $2.7 \mathrm{~Hz}$ modes are close to 0.8 , which confirms the existence of strong linear correlation with temperature. The standard errors of the linear fit for these two vibration modes are around $0.55 \%$ of the mean, which is not far off from the baseline standard deviations for these two modes, as found in Table 3-1. This result suggests that once the temperature effect has been taken into account, 
the remaining variability of the frequencies is mainly due to numerical sources of uncertainty (i.e. those that cause the baseline variability) as opposed to additional physical sources of uncertainty different from temperature.

Another observation from the frequency vs. temperature plots is that the slope of the linear trend seems to become more pronounced as the modal frequency increases. To show this, Table 3-3 gives values of change in modal frequency due to a $45^{\circ} \mathrm{C}$ change in temperature, which is approximately the range of fluctuation of average concrete temperatures observed over a six-month period. As an example, a $45^{\circ} \mathrm{C}$ temperature increment would produce a change of around $1.7 \%$ in the extracted frequency of the $0.47 \mathrm{~Hz}$ mode while producing a change of around $3.6 \%$ in the frequency of the $2.7 \mathrm{~Hz}$ mode.

Correlation of the modal frequencies with other variables such as cross-section temperature gradients, wind speed and vibration amplitude were investigated by including the variables as additional independent variables in linear regressions. The different regression attempts did not suggest significant correlations of the modal frequencies to the other variables, except for some slight correlation of the modal frequencies of the lower modes with wind speed. The results of linear regressions in which wind-speed squared is considered as an additional independent variable may be found in Table 3-3. It may be observed that by taking the wind speed into account there seems to be a slight improvement in the correlation coefficients of the $0.47 \mathrm{~Hz}$ and the $1.6 \mathrm{~Hz}$ modes, while no significant improvement in the correlation is obtained for the other two higher frequency modes. It makes sense that the wind affects more significantly the wind-driven modes, such as the 0.47 $\mathrm{Hz}$ lateral mode, than other non wind-driven modes such as the $2.7 \mathrm{~Hz}$ mode. However, it is recognized that the observed correlation of the modal frequencies with wind speed is not 
strong or clear enough to be entirely conclusive regarding the influence of the wind on the modal frequencies.

\subsection{Variability of modal parameters vs. sensitivity to damage}

From the viewpoint of practical structural health monitoring it is desirable to establish whether the variability in the extracted modal parameters represents a significant challenge or not for the implementation of damage detection algorithms. In particular, an answer is required for the question of what level or extent of damage does it take for the damage to be detectable using vibration-based techniques? This section addresses this question by comparing the damage sensitivity of the Confederation Bridge based on finite element model simulations of different potential damage scenarios with the actual observed variability results presented in the previous sections. The finite element model used for the damage sensitivity simulations is shown in Figure 2-6(b). This beam element model has been previously verified and calibrated based on the measured data, as discussed in Chapter 2 . Two different types of damage scenario have been simulated: (i) uniform pier stiffness degradation, which models the type of deterioration potentially arising from long-term immersion in saltwater; and (ii) localized damage occurring at the cast-in-place prestressed joints of one of the continuous drop-in spans. These joints are made continuous by posttensioning pre-stress and grouting. As an example, partial loss in continuity and in stiffness at these joints could be triggered by ruptures of the pre-stressing tendons that give continuity to the joints. The locations of simulated damage are shown in Figure 3-18.

Pier stiffness degradation is modeled by means of uniform reduction in the elastic modulus of pier elements, while partial damage occurring at the prestressed joints of the continuous drop-in span is modeled by a reduction in the inertias of joint elements. Different levels of stiffness reduction are considered for each of the two damage scenarios. Reductions 
of pier stiffness of $2 \%, 5 \%$ and $10 \%$ are considered, while inertia reductions of $50 \%$ and $75 \%$ are considered for the joint elements of the continuous drop-in span, resulting in the simulation of significant damage occurring at these locations.

In this sensitivity study, which is intended to give a preliminary indication of the feasibility of using vibration based health monitoring in the field, damage sensitivity is evaluated directly by means of the changes in modal frequencies and mode shapes. Evaluations of damage sensitivity using damage detection indices such as those based on modal curvatures or modal flexibility (e.g. as considered by Wang et al. 2000) are beyond the scope of the present preliminary study. It is recognized that those damage detection methods are expected to be more sensitive to damage than the changes in the modal parameters alone. Still, the results presented here serve as an initial overview and quantitative assessment of the challenges that the variations in the modal parameters identified from long-term monitoring data may represent for conducting damage detection in practice.

In some of the damage simulations considered here, the sequence of the vibration modes can have slight changes once the damage scenarios are introduced, with some vibration modes switching places with other close modes. Therefore appropriate damagedundamaged mode pairs have to be identified before performing comparisons of the modal properties obtained before and after damage. Here, the appropriate mode pairs are found by means of the modal assurance criterion (MAC).

\subsubsection{Results - pier stiffness degradation scenarios}

The results of the modal frequency and mode-shape sensitivity analyses are shown in Figures 3-19 to 3-23. The bars in Figure 3-19 show the changes in the modal frequencies with respect to the original values obtained from the finite element model after introducing the simulated damage scenarios. It may be observed from this figure that the patterns of 
damage sensitivity of the modal frequencies to the pier degradation and joint damage scenarios are quite different from each other. The joint damage scenarios seem to affect a few modes more dramatically than others as compared to the scenarios of pier stiffness degradation, which tend to significantly affect a larger number of vibration modes.

From Figure 3-19, it seems that a pier stiffness degradation of $2 \%$ would be difficult to detect from long-term vibration monitoring data because the resulting modal frequency changes are all either below or very close to the baseline variability level of $0.5 \% .{ }^{4}$ For similar reasons, as may be observed from Figure 3-20, it seems that changes in the mode shapes are not likely to serve as indicators of this level of damage either.

On the other hand, a pier stiffness degradation of $5 \%$ seems more feasible to detect because the modal frequency changes of 27 out of 50 modes exceed the baseline variability level while 18 out of 50 exceed the general variability level, with some modes approaching a $1.5 \%$ reduction in modal frequency, which significantly exceeds the general variability level of $1.1 \%$. Moreover, if temperature effects were to be removed from the extracted frequencies then the changes resulting from damage would stand out further above the general variability, which would be lowered to around $0.7 \%$ on average (from Table 3-3).

As may be observed in Figure 3-19, a pier stiffness deterioration scenario of $10 \%$ stiffness reduction would produce clearly noticeable effects on the modal frequencies. The changes of several modal frequencies would exceed the general variability level of extracted frequencies by a factor of 2 or more. Changes in the mode shapes of the $5.57 \mathrm{~Hz}$ and 5.70 $\mathrm{Hz}$ vibration modes could also potentially indicate this type of damage, as shown in Figure

\footnotetext{
${ }^{4}$ Herein forth, 'variability level' refers to the average standard deviations for frequencies extracted from the monitoring data and the average $\mathrm{MAC}$ values for extracted mode-shapes.
} 
3-20. The mode shapes of these two vibration modes before and after the introduction of the damage are shown in Figure 3-22. However these higher frequency lateral modes are not normally well excited under typical operational loading conditions, so detecting damage based on the changes of these two particular mode shapes would be difficult in practice.

\subsubsection{Results - damage at continuous drop-in span joints}

As may be observed in Figure 3-19(b), the modal frequencies of a few vibration modes appear to be much more sensitive to this kind of damage scenario than the modal frequencies of other modes. In particular, the modal frequency most sensitive to damage at the joints of the continuous drop-in span is that of the $1.78 \mathrm{~Hz}$ mode. This theoretical mode actually corresponds to the experimentally extracted $1.82 \mathrm{~Hz}$ mode presented earlier in this chapter. By looking at the mode shape in Figure 3-6, the reason why this mode is so particularly sensitive to damage at these joints becomes evident. The reason is that the mode shape involves large rotations precisely at the joint locations. Despite the relatively high damage sensitivity of this mode, the change in its modal frequency after a $50 \%$ reduction in the inertia of the joints, which is a significant level of damage, is not enough to exceed the general variability level of extracted frequencies. This means that a stiffness degradation of $50 \%$ could easily be masked by the variability and would be difficult to detect from changes in the modal frequencies. For damage occurring at the prestressed joints to become easily detectable from the changes in modal frequencies a more advanced stage of stiffness degradation seems to be necessary.

A damage scenario involving an inertia reduction of $75 \%$ would produce significantly more noticeable changes in the modal frequencies, as shown in Figure 3-19(b). It may be argued however that by the time a $75 \%$ stiffness reduction is reached at this girder joint, the damage would have probably become evident already through the appearance of large cracks. 
In regards to the mode-shape changes, as observed from Figure 3-21, there are two mode-shapes which are particularly sensitive to damage at the continuous span joints, namely the shapes of the 4.81 and $4.91 \mathrm{~Hz}$ modes, which are depicted in Figure 3-23 before and after damage. Both of these higher vertical vibration modes have been observed to be routinely excited under operational loadings with heavy traffic and they are easily identifiable from the monitoring data. From a practical perspective this means that these two modes can potentially serve as indicators of this kind of structural damage, i.e. damage occurring at the joints of the continuous drop-in span. It is worth noting that the mode shape of the $1.78 \mathrm{~Hz}$ mode, which exhibited the highest modal frequency sensitivity to this damage scenario, is not found to be sensitive to damage.

\subsection{Variability model}

\subsubsection{Amplitude dependent mode shape variability model}

From the analysis of the monitoring data it is observed that the variability of modal amplitudes is significantly higher for sensor locations with low modal amplitudes than for sensor locations with high amplitudes. This is as expected since higher amplitude signals tend to have better signal-to-noise ratios. Figure 3-24 shows the standard deviation as function of modal amplitude for the baseline and general datasets respectively. The standard deviations are shown as a fraction of the mean modal amplitude. The modal amplitudes are normalized by the amplitude of a reference sensor location. The data are fitted with second order rational polynomials, which give R-square values of 0.84 and 0.82 respectively. The equations for each of the two models are as follows:

$$
s_{i}^{b}(\%)=\frac{3.414}{\psi_{i}^{n^{2}}+0.9524 \psi_{i}^{n}+0.04748} \quad \text { (Baseline datasets) }
$$




$$
s_{i}^{g}(\%)=\frac{2.774}{\psi_{i}^{n^{2}}+0.2131 \psi_{i}^{n}+0.04871} \quad \text { (General datasets) }
$$

where $s_{i}(\%)$ is the standard deviation as percentage of the mean normalized modal amplitude $\psi_{i}^{n}$. Table 3-5 gives a few representative values of standard deviations as given by the above equations. The value of $s_{i}^{b}$ given by Eq. (3-4) is cut-off at $51 \%$ for very small amplitudes (normalized amplitudes lower than 0.02). This is to prevent $s_{i}^{b}$ from exceeding $s_{i}^{g}$, which is not realistic and would generate problems in the simulations, where it is assumed that $s^{g}>s^{b}$. The above models will be used to simulate the variability of the mode shapes in the damage detection simulations of Chapter 6 .

The implications for damage detection of the amplitude dependent variability of the modal amplitudes is that stiff structural members or stiff regions of the structure, such as the piers in the valley bridge example of Chapter 6 , which tend to have low modal amplitudes for most lower frequency mode shapes, will inevitably be more heavily contaminated with noise than other more flexible regions of the structure (such as the girders in the valley bridge example). This leads to lower accuracy in the damage detection results in the stiffer regions of the model.

In practice one way to minimize this undesirable effect would be to use higher sensitivity sensors in these stiffer regions of the structure and to maximize the resolution of the analogue-to-digital conversion of the measured signals in these regions by setting the voltage measurement ranges as narrow as possible.

\subsubsection{Noise simulation for damage detection}

In comparative damage detection methods, including damage detection via finite element updating, the structural properties extracted from a reference sample or set of 
measurements, representing the "undamaged" state of the structure, are compared against the structural properties extracted from another sample of measurements obtained under the "damaged" state (or suspected damage state) of the structure for evaluation. The calibration of the structural model and damage detection steps of the FE updating method are illustrated in Figure 3-25.

A set of measurements - i.e. a "sample" - taken at the initial undamaged state may be expressed as follows

$$
\begin{aligned}
& x_{1}^{u m}=x^{u}+n_{1}^{u m} \\
& x_{2}^{u m}=x^{u}+n_{2}^{u m} \\
& \vdots \\
& x_{N_{1}}^{u m}=x^{u}+n_{N_{1}}^{u m}
\end{aligned}
$$

where $x$ represents a structural property (e.g. either an eigenfrequency or the modal coefficient of a given mode shape), $x_{i}^{t m}$ is the $i$-th measured value of the undamaged structure, $x^{u}$ is the true value of the undamaged structure, $n_{i}^{u m}$ represents the measurement noise in the $i$-th measurement, and $N_{1}$ represents the number of measurements. Given that each sample consists of multiple independent measurements or observations, the effect of measurement error on the identification of structural properties of both damaged and undamaged states can be reduced by averaging. An average un-damaged measurement can be written as follows

$$
\bar{x}^{u m}=x^{u}+\bar{n}^{u m}
$$

Assuming $n_{i}^{u m}$ has a normal distribution with zero mean and a variance of $\sigma_{m}^{2}$, then its average $\bar{n}^{u m}$ also has zero mean and a variance of $\sigma_{\overline{u m}}^{2}=\frac{\sigma_{m}^{2}}{N_{1}}$ (Scheaffer et al. 1982). The field measured "baseline" standard deviation $s_{b}$ can be used as an estimate of $\sigma_{m}^{2}$, i.e. $\hat{\sigma}_{m}^{2}=s_{b}^{2}$. 
Similarly, the sample or set of measurements taken from the "damaged" structure can be written as follows

$$
\begin{aligned}
& x_{1}^{d m}=x^{d}+\Delta x^{e}+n_{1}^{d m}=\left(x^{u}+\Delta x^{d}\right)+\Delta x^{e}+n_{1}^{d m} \\
& x_{2}^{d m}=x^{d}+\Delta x^{e}+n_{2}^{d m}=\left(x^{u}+\Delta x^{d}\right)+\Delta x^{e}+n_{2}^{d m} \\
& \vdots \\
& x_{N_{2}}^{d m}=x^{d}+\Delta x^{e}+n_{N_{2}}^{d m}=\left(x^{u}+\Delta x^{d}\right)+\Delta x^{e}+n_{N_{2}}^{d m}
\end{aligned}
$$

where $x_{i}^{d m}$ is the $i$-th measured value of the damaged structure, $x^{d}$ is the true value of the structural property of the damaged structure, $\Delta x^{e}$ is the change in the structural property due to the environment and loading conditions, assumed to be constant over the relatively short time period of sample data collection, $\Delta x^{d}$ is the change in the structural property due to damage, $n_{i}^{d m}$ is the measurement noise in the $i$-th measurement, and $N_{2}$ is the number of measurements.

Again, averaging of the field measured data of the damaged structure is carried out to reduce the effect of the measurement noise. The average measurement of the damaged structure is expressed as follows

$$
\bar{x}^{d m}=x^{u}+\Delta x^{d}+\Delta x^{e}+\bar{n}^{d m}
$$

Assuming the measurement noise $n_{i}^{d m}$ has the same distribution as $n_{i}^{u m}$, i.e. a normal distribution with zero mean and a variance $\sigma_{m}^{2}$, the average measurement noise of the damaged structure $\bar{n}^{d m}$ also has a normal distribution with zero mean and a variance of $\sigma_{\overline{d m}}^{2}=\frac{\sigma_{m}^{2}}{N_{2}}$

The difference between the two sample mean structural properties is a combination of the change caused by damage (if any), and a variability component resulting from changes due to the environment and measurement noises, as follows 


$$
\bar{x}^{d m}-\bar{x}^{u m}=\Delta x^{d}+n^{d}
$$

where $n^{d}=\Delta x^{e}+\bar{n}^{d m}-\bar{n}^{\imath m}$ is the total noise on the comparative damage detection.

If $\Delta x^{e}$ is assumed to be normally distributed with zero mean and a variance of $\sigma_{e}^{2}$, and $\Delta x^{e}$, $\bar{n}^{d m}$ and $\bar{n}^{u m}$ are assumed to be mutually independent, then $n^{d}$ also has a normal distribution with zero mean and variance

$$
\sigma_{d}^{2}=\sigma_{e}^{2}+\sigma_{\overline{d m}}^{2}+\sigma_{u m}^{2}
$$

From the "general" variability datasets of the variability study presented in the previous section, the noise $n_{g}$ in each of the 22 measurements of the structural properties is composed of an environmental component and a measurement noise component $n^{\text {m }}$, which can be expressed as follows

$$
n_{g}=\Delta x^{e}+n^{n}
$$

The measurement noise term $n^{m}$ may be assumed to have the same distribution as $n_{i}^{u m}$ and $n_{i}^{d m}$, i.e. a normal distribution with zero mean and a variance of $\sigma_{m}^{2}$. Assuming $\Delta x^{e}$ and $n^{m}$ are independent, then $n_{g}$ is also normally distributed with zero mean and a variance of

$$
\sigma_{g}^{2}=\sigma_{e}^{2}+\sigma_{m}^{2}
$$

Substituting Eq. (3-13) in (3-11),

$$
\sigma_{d}^{2}=\sigma_{g}^{2}-\sigma_{m}^{2}+\sigma_{\overline{d m}}^{2}+\sigma_{u m}^{2}=\sigma_{g}^{2}-\sigma_{m}^{2}+\frac{\sigma_{m i}^{2}}{N_{2}}+\frac{\sigma_{m}^{2}}{N_{1}}
$$

Noting that the field measured standard deviation $s_{g}$ of the structural properties extracted from the "general" datasets can be used as an estimate of $\sigma_{g}^{2}$, i.e. $\hat{\sigma}_{g}^{2}=s_{g}^{2}$, and that $\hat{\sigma}_{m}^{2}=s_{b}^{2}$, Eq. (3-14) can be written in terms of variance estimates obtained from the monitoring data as 


$$
\hat{\sigma}_{d}^{2}=s_{d}^{2}=s_{g}^{2}-s_{b}^{2}+\frac{s_{b}^{2}}{N_{2}}+\frac{s_{b}^{2}}{N_{1}}
$$

Therefore,

$$
\hat{n}^{d} \in N\left(0, s_{g}^{2}+s_{b}^{2}\left(\frac{1}{N_{1}}+\frac{1}{N_{2}}-1\right)\right)
$$

Using Eq. (3-16) the noise effects of random environmental fluctuations and measurement noises encountered in practice on damage detections using measured structural properties can be simulated more realistically than in other previous studies of purely theoretical computer simulations. In damage detection simulations here, the noise in the monitoring data representative of the field measured variability is introduced as follows

$$
x^{s d m}=x^{d}+r \cdot n \cdot\left(0, s_{d}^{2}\right)
$$

where $x^{s d m}$ is the simulated "measured" value of a modal frequency or modal amplitude of the damaged structure, $x^{d}$ is the corresponding true value of the modal property obtained from the finite element model of the damaged structure, and $r n .\left(0, s_{d}^{2}\right)$ is a random number taken from a normal distribution with zero mean and a variance of $s_{d}^{2}$. The added noise is a lumped noise that accounts for the measurement and environmental noises on the sample mean values of both the undamaged and damaged structures. For the mode shapes, since the standard deviations are amplitude dependent, $s_{b}$ and $s_{g}$ are obtained from Eqs. (3-4) and (3-5), respectively.

By including the amplitude dependent variability, the model improves significantly from earlier damage detection simulation studies which typically assume a uniform random noise in the simulation of the "measured" mode shape amplitudes. Normally, the mode shape amplitudes are assumed to have random errors with a constant variance (as \% of the mode shape amplitude), of typically $2 \%$ or $5 \%$ (e.g. Humar et al. 2006). Based on the 
observations from the monitoring of the Confederation Bridge, this may not be an appropriate representation of the behaviour of actual field data.

Although other environmental factors such as humidity, sunlight direction and other possible factors may also contribute to the variation of the vibration properties of the Confederation Bridge, it is assumed in this benchmark study that the environmentally induced variability in the eigenfrequencies is due mainly to the effect of temperature. By applying frequency versus temperature regressions, the influence of the environmental variation can be eliminated from the eigenfrequency results, reducing $s_{g}$ to $s_{b}$. Hence, the variance of the noise $n^{d}$ in the eigenfrequencies is reduced, as follows

$$
s_{d f}^{2}=s_{b}^{2}\left(\frac{1}{N_{1}}+\frac{1}{N_{2}}\right)
$$

This value is used for the eigenfrequencies in Eq. (3-17) - instead of $s_{d}$ - in the damage detection simulations of Chapter 6 .

The FE updating residuals that would normally be encountered in practice are represented in the damage detection simulations by the inevitable updating residuals that result from the fact that the damage functions cannot exactly match the actual damage.

\subsection{Conclusions}

Vibration based structural health monitoring could potentially become a useful decision support tool to optimize the allocation of limited infrastructure maintenance resources, helping to curb the rising trend of infrastructure maintenance costs, as well as in providing timely information for post-disaster recovery operations after the occurrence of extreme events such as earthquakes, hurricanes, etc. Vibration based damage detection normally relies on detecting changes in the static or dynamic structural properties extracted 
from measured vibration response data. The main problem when attempting to apply these techniques to real structures in the field is that the changes in the dynamic properties arise not only from damage but also from various numerical and physical sources of uncertainty. Under such conditions, the changes caused by damage must be distinguished from the changes arising from the normal variability making the damage detection problem much more challenging. In order to overcome this difficulty it is necessary to understand the root causes and characteristics of the variability and also to quantify how the variability compares to the changes caused by damage, which have been the objectives of this chapter.

The investigation presented here is based on the field monitoring data from the Confederation Bridge monitoring project, in Canada. The location of the bridge in the harsh environment of the Northumberland Strait together with the availability of long-term continuous monitoring covering different relevant structural and environmental variables data makes it the ideal setting for this investigation.

In the first part of this study the sources of uncertainty in the structural parameters extracted from the measured structural responses have been classified into two major categories: (I) numerical uncertainties and (II) physical uncertainties. Subsequently, an attempt has been made to quantify the variability arising from each of these sources separately. First, the variability arising from the numerical uncertainties, referred to here as the baseline variability, is quantified from the analysis of datasets collected over short period of time under similar loading and environmental conditions, so as to minimize the physical uncertainties. It is found that, under such conditions, extracted modal frequencies exhibit average standard deviations of $0.5 \%$ from the mean, while extracted mode-shapes exhibit an overall average modal assurance criterion (MAC) value of 0.988 , with modal amplitudes having an average standard deviation of $3.9 \%$ from the mean. On the other hand, the 
identified damping ratios exhibit a much larger variability, with discrepancies between extracted values that can sometimes be close to a factor of 2 and with standard deviations of around $35 \%$ from the mean values. Nonetheless, the results demonstrate that it is possible to retrieve relatively consistent modal frequencies and mode-shapes from monitoring datasets under similar loading scenarios and environmental conditions.

Having determined the baseline level of variability, the study proceeds to an investigation of the general variability of extracted structural parameters identified in the presence of both numerical and physical uncertainties by analyzing data from the Confederation Bridge collected over a 6-month period under typical random environmental and loading fluctuations. It is found that the standard deviations of extracted modal frequencies under these conditions are on average around $1.1 \%$ from the mean, which is roughly a factor of 2 times greater than the baseline standard deviations. This means that for the Confederation Bridge approximately half of the variability of the identified eigenfrequencies is attributable to the environmental and loading fluctuations and the other half is attributable to the numerical uncertainties. The mode shapes also exhibit significantly more variability under the general scenarios with average MAC values decreasing to 0.968 , and average standard deviations of the modal amplitudes increasing to $5.5 \%$ from the mean values. On the other hand, the variability of damping ratios remains roughly at the same level, with standard deviations around $33 \%$ from the mean.

Correlations of the observed variability with variables such as temperature and wind speed are investigated. Average concrete temperature is found to correlate strongly with the extracted modal frequencies. For the Confederation Bridge, this relation seems to approximate a linear inverse trend with the effect of temperature becoming more pronounced and less dispersion observed in the trend for the higher frequency modes. In 
particular, a $45^{\circ} \mathrm{C}$ temperature increment would produce a reduction of around $1.7 \%$ in the extracted frequency of the $0.48 \mathrm{~Hz}$ mode while producing a reduction of around $3.6 \%$ in the frequency of the $2.7 \mathrm{~Hz}$ mode. The changes in modal frequencies attributable to the temperature fluctuations explain most of the variability additional to the baseline variability, especially for the higher frequency modes.

Additionally, the feasibility of implementing vibration based damage detection techniques in practice is evaluated. For this purpose, a damage sensitivity study based on a finite element model of the Confederation Bridge is conducted to determine the expected changes in modal parameters of the bridge resulting from two different potential damage scenarios. The changes are compared to the observed standard deviations of modal parameters extracted from the field monitoring data. The results suggest that detecting damage at early stages would be difficult because the corresponding changes in modal properties tend to be comparable or below the variability of modal parameters extracted from the monitoring data. As a result, the changes in the structural indices used for damage detection could easily be masked by the normal variations of the data, especially at the early stages of damage, which are of most practical interest. This issue, which is central in determining the feasibility of practical structural health monitoring, is considered in greater depth in Chapter 6, where the ability of advanced damage detection methods to detect damage in the presence of realistic levels of variability is evaluated by means of realistic damage detection simulations.

To consider the variability of the field monitoring data in the damage detection simulations as faithfully to the observed behaviour as possible, a model for the simulation of variability has been developed and presented. The model accounts for the following factors: 
1. The amplitude dependence of the variability of identified mode shape coefficients as observed from the Confederation Bridge data. By including this, the model improves significantly upon the traditional practice of assuming a constant variance (\%) noise for the mode shape amplitudes in damage detection simulation studies.

2. Averaging of multiple measurements to form sample average values with reduced variability.

3. Contributions from both the measurement errors and the environmental fluctuations (i.e. from the baseline and general variability) in the measured "undamaged" and "damaged" modal properties. The model considers the total combined variability involved in the comparison of "undamaged" vs. "damaged" modal properties.

\subsection{Acknowledgements}

Funding support and assistance provided to the monitoring project from the National Science and Engineering Research Council (NSERC) through a CRD research grant, Public Works and Government Services Canada (PWGSC), and Strait Crossing Bridge Ltd. (SCBL) are gratefully acknowledged. Technical information and assistance was provided by Alain Solari and John Egan at PWGSC and Don McGinn at SCBL. The finite element model was developed by Dr. W.C. Li, post-doctoral fellow at the Ottawa-Carleton Bridge Research Institute, Carleton University. 
Table 3-1: Summary of baseline variability of extracted modal parameters

\begin{tabular}{ccccccc}
\hline $\bar{f}$ & $\begin{array}{c}\text { Average } \\
\text { Model Order }\end{array}$ & $\begin{array}{c}\text { Variability } \\
\text { range }(\%)\end{array}$ & $\begin{array}{c}\sigma_{f} \\
(\% \text { of } \bar{f})\end{array}$ & $\bar{\xi}$ & $\begin{array}{c}\sigma_{\xi} \\
(\% \text { of } \bar{\xi})\end{array}$ & $\overline{M A C}$ \\
\hline 0.4759 & 41 & 1.3 & 0.3818 & 0.0165 & 27 & 0.9887 \\
0.4758 & 64 & 1.3 & 0.4082 & 0.0164 & 26 & 0.9876 \\
1.6373 & 39 & 1.7 & 0.6090 & 0.0186 & 40 & 0.9877 \\
1.6351 & 82 & 1.9 & 0.6735 & 0.0167 & 38 & 0.9886 \\
1.8210 & 39 & 1.2 & 0.3690 & 0.0180 & 35 & 0.9931 \\
1.8212 & 72 & 1.1 & 0.3609 & 0.0171 & 34 & 0.9880 \\
2.7735 & 42 & 1.9 & 0.5312 & 0.0140 & 40 & 0.9843 \\
2.7734 & 74 & 2.5 & 0.7142 & 0.0125 & 38 & 0.9831 \\
\hline Averages & - & 1.6 & 0.5060 & 0.0162 & 35 & 0.9876 \\
\hline
\end{tabular}


Table 3-2: Summary of variability of modal parameters over 6-month period.

\begin{tabular}{c|cccccc}
\hline $\bar{f}$ & $\begin{array}{c}\text { Average } \\
\text { model order }\end{array}$ & $\begin{array}{c}\text { Variability } \\
\text { range } \%)\end{array}$ & $\begin{array}{c}\sigma_{f} \\
(\% \text { of } \\
\bar{f})\end{array}$ & $\begin{array}{c}\bar{\xi} \\
\left(\begin{array}{c}\% \\
\bar{\xi} \text { of }\end{array}\right.\end{array}$ & $\overline{M A C}$ \\
\hline 0.4739 & 39 & 3.6 & 0.95 & 0.021 & 29 & 0.974 \\
0.4735 & 61 & 3.7 & 0.94 & 0.021 & 28 & 0.971 \\
1.6267 & 36 & 4.4 & 1.19 & 0.022 & 45 & 0.984 \\
1.6256 & 60 & 4.7 & 1.27 & 0.022 & 43 & 0.975 \\
1.8161 & 36 & 3.5 & 1.06 & 0.015 & 24 & 0.973 \\
1.8181 & 60 & 3.4 & 1.11 & 0.015 & 28 & 0.937 \\
2.7557 & 36 & 3.5 & 1.13 & 0.015 & 33 & 0.968 \\
2.7531 & 59 & 3.7 & 1.22 & 0.012 & 38 & 0.966 \\
\hline Averages & & 3.8 & 1.11 & 0.018 & 33 & 0.968 \\
\hline
\end{tabular}


Table 3-3: Linear regression results.

\begin{tabular}{|c|c|c|c|c|c|c|c|c|}
\hline & \multicolumn{4}{|c|}{ Linear Regression $f(T)$} & \multicolumn{4}{|c|}{ Linear Regression $f\left(T, W S^{2}\right)$} \\
\hline $\bar{f}$ & $\begin{array}{l}\text { T slope } \\
{\left[\mathrm{Hz} /{ }^{\circ} \mathrm{C}\right]}\end{array}$ & $\mathrm{r}^{2}$ & $\begin{array}{c}\text { Standard } \\
\text { Error } f[\% \\
\text { of } \bar{f}]\end{array}$ & $\begin{array}{c}\Delta f \text { due to } \\
45^{\circ} \mathrm{C} \Delta T \\
{[\%]}\end{array}$ & $\begin{array}{c}\text { T slope } \\
{\left[\mathrm{Hz} /{ }^{\circ} \mathrm{C}\right]}\end{array}$ & $\begin{array}{l}\mathrm{WS}^{2} \text { slope } \\
{\left[\mathrm{Hz} /\left(\mathrm{m} / \mathrm{s}^{2}\right)^{2}\right]}\end{array}$ & $r^{2}$ & $\begin{array}{c}\text { Standard } \\
\text { Error } f \\
{[\% \text { of }} \\
\bar{f}_{]}\end{array}$ \\
\hline 0.4739 & -0.00017 & 0.23 & 0.85 & -1.58 & -0.0001 & 0.000013 & 0.38 & 0.78 \\
\hline 0.4735 & -0.00018 & 0.29 & 0.81 & -1.75 & -0.0001 & 0.000012 & 0.41 & 0.76 \\
\hline 1.6267 & -0.00091 & 0.37 & 0.97 & -2.51 & -0.0012 & -0.000053 & 0.49 & 0.89 \\
\hline 1.6256 & -0.00101 & 0.41 & 1.00 & -2.79 & -0.0012 & -0.000034 & 0.45 & 0.99 \\
\hline 1.8161 & -0.00131 & 0.80 & 0.49 & -3.26 & -0.0014 & -0.000019 & 0.81 & 0.48 \\
\hline 1.8181 & -0.00133 & 0.73 & 0.59 & -3.29 & -0.0014 & -0.000010 & 0.74 & 0.60 \\
\hline 2.7557 & -0.00209 & 0.77 & 0.55 & -3.41 & -0.0025 & -0.000060 & 0.83 & 0.48 \\
\hline 2.7531 & -0.00230 & 0.80 & 0.56 & -3.76 & -0.0027 & -0.000063 & 0.86 & 0.48 \\
\hline Averages & & 0.55 & 0.73 & & & & 0.62 & 0.68 \\
\hline
\end{tabular}


Table 3-4: Standard deviations of mode shape amplitudes. Values are given as percentage of the mean amplitude of the corresponding modal coefficient. Both baseline values and general values under random environmental fluctuations are given (the latter in parentheses).

\begin{tabular}{|c|c|c|c|c|c|c|c|c|c|c|c|c|c|c|c|}
\hline & & & & & & & $D O F$ & & & & & & & & \\
\hline & $5 \mathrm{~V} 1$ & $5 \mathrm{~V} 2$ & $5 \mathrm{~T} 1$ & $6 \mathrm{~V} 1$ & $6 \mathrm{~V} 2$ & 6T1 & $7 \mathrm{~V} 1$ & $7 \mathrm{~V} 2$ & $7 \mathrm{~T} 1$ & 8V1 & $8 \mathrm{~V} 2$ & $8 \mathrm{~T} 1$ & $9 \mathrm{~V} 1$ & 9V2 & $9 \mathrm{~T} 1$ \\
\hline $\begin{array}{l}\text { Mode } \\
\text { freq }\end{array}$ & & & & & & & & & & & & & & & \\
\hline 0.47 & - & - & $\begin{array}{l}8.6 \\
(19)\end{array}$ & - & - & $\begin{array}{l}7.9 \\
(15)\end{array}$ & - & - & $\begin{array}{c}1.9 \\
(3.5)\end{array}$ & - & - & $\begin{array}{c}0.9 \\
(2.7)\end{array}$ & - & - & $\begin{array}{l}1.3 \\
(3.1)\end{array}$ \\
\hline 1.63 & $\begin{array}{l}7.9 \\
(16)\end{array}$ & $\begin{array}{l}8.4 \\
(17)\end{array}$ & - & $\begin{array}{c}11 \\
(14)\end{array}$ & $\begin{array}{c}11 \\
(13)\end{array}$ & - & $\begin{array}{l}2.2 \\
(1.8)\end{array}$ & $\begin{array}{c}2.1 \\
(1.6)\end{array}$ & - & $\begin{array}{l}0.65 \\
(1.7)\end{array}$ & $\begin{array}{l}0.67 \\
(1.1)\end{array}$ & 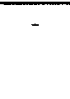 & $\begin{array}{l}46 \\
(61)\end{array}$ & $\begin{array}{c}50 \\
(46)\end{array}$ & - \\
\hline 1.82 & $\begin{array}{c}11 \\
(13)\end{array}$ & $\begin{array}{c}12 \\
(13)\end{array}$ & - & $\begin{array}{c}28 \\
(35)\end{array}$ & $\begin{array}{c}33 \\
(30)\end{array}$ & - & $\begin{array}{c}1.4 \\
(2.7)\end{array}$ & $\begin{array}{c}1.1 \\
(2.5)\end{array}$ & - & $\begin{array}{c}0.69 \\
(0.84)\end{array}$ & $\begin{array}{l}0.45 \\
(1.0)\end{array}$ & - & $\begin{array}{l}19 \\
(35)\end{array}$ & $\begin{array}{c}19 \\
(33)\end{array}$ & 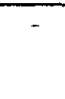 \\
\hline 2.57 & $\begin{array}{c}27 \\
(51)\end{array}$ & $\begin{array}{c}25 \\
(53)\end{array}$ & - & $\begin{array}{c}6.1 \\
(5.6)\end{array}$ & $\begin{array}{c}5.3 \\
(5.5)\end{array}$ & - & $\begin{array}{r}2.2 \\
(2.7)\end{array}$ & $\begin{array}{c}2.1 \\
(2.3)\end{array}$ & - & $\begin{array}{c}36 \\
(46)\end{array}$ & $\begin{array}{c}30 \\
(47)\end{array}$ & - & $\begin{array}{c}1.7 \\
(2.4)\end{array}$ & $\begin{array}{l}2.8 \\
(2.3)\end{array}$ & - \\
\hline
\end{tabular}


Table 3-5: Values of variability of mode shape amplitudes as predicted by rational polynomial least square fits.

\begin{tabular}{|c|c|c|}
\hline $\begin{array}{c}\text { Normalized mode } \\
\text { shape amplitude }\end{array}$ & $\begin{array}{c}\text { Standard deviation of the modal amplitude } \\
\text { (\% of mean) }\end{array}$ \\
\hline 0.1 & Baseline Datasets & General Datasets \\
\hline 0.5 & 22 & 35 \\
\hline 1 & 4.4 & 6.8 \\
\hline 1.25 & 1.7 & 2.2 \\
\hline
\end{tabular}




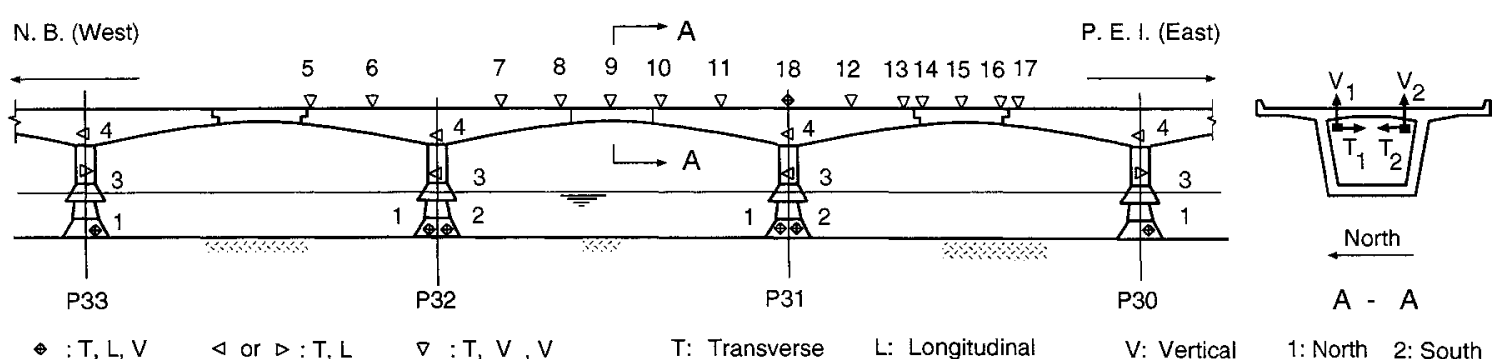

Figure 3-1: Locations of accelerometers in Confederation Bridge monitoring system. 


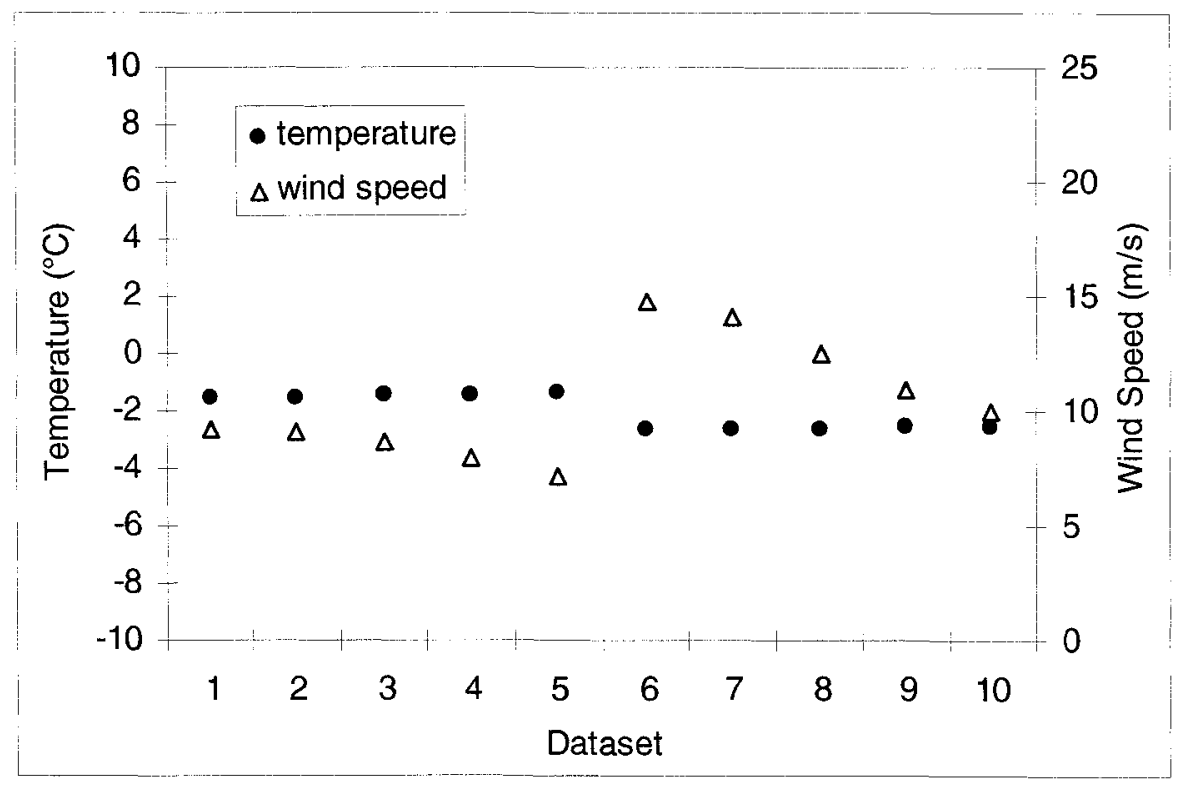

Figure 3-2: Environmental conditions of baseline variability datasets. 


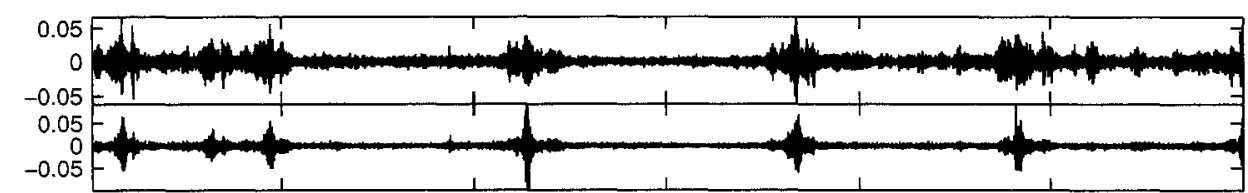

$7 \mathrm{~V} 1$

03/2003

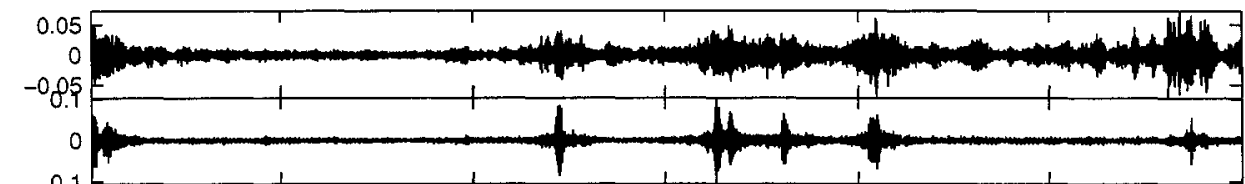

$7 \mathrm{~V} 1$

7T1 12:42

$13 / 03 / 2003$

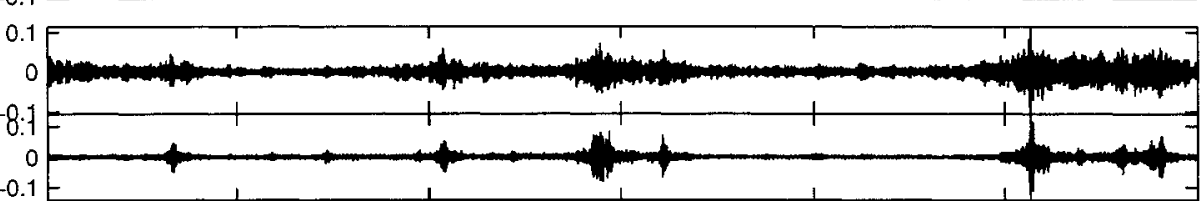

$7 \mathrm{~V} 1$

$13 / 03 / 2003$

7T1 12:52

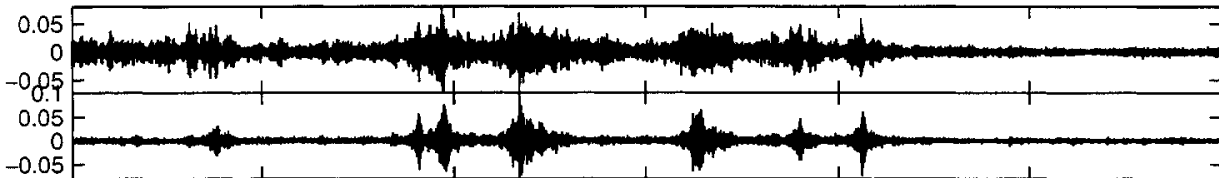

$7 \mathrm{~V} 1$

$13 / 03 / 2003$

7T1 13:02

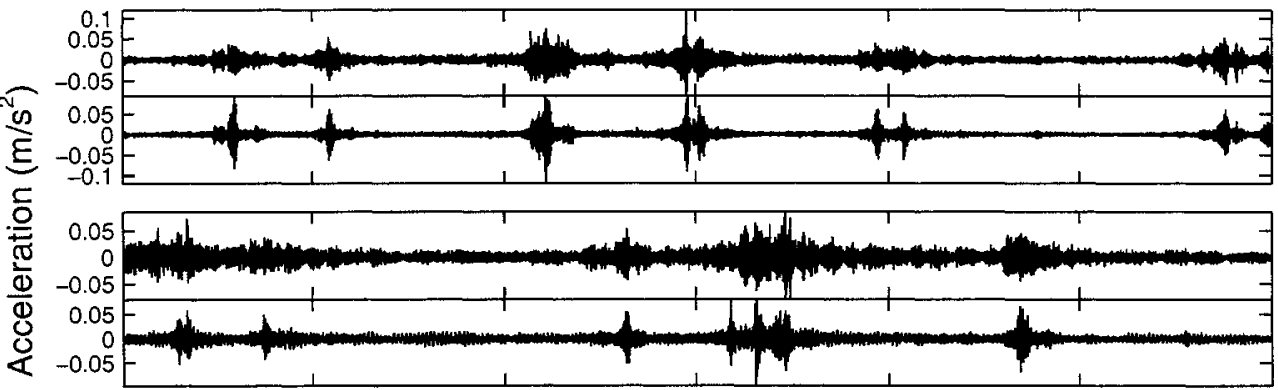

$7 V_{1}$

$13 / 03 / 2003$

7T1 13:12

$7 V_{1}$

$14 / 03 / 2003$

$7 T 1$ 12:07

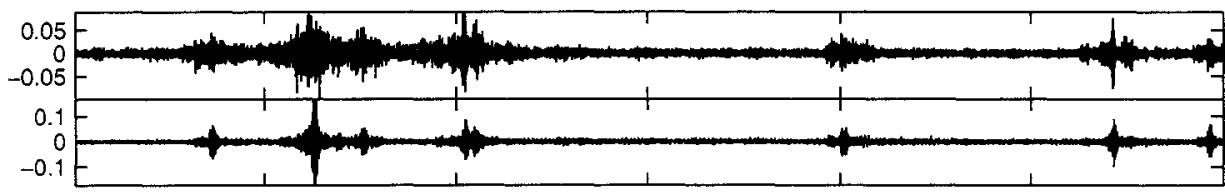

$7 \mathrm{~V} 1$

$14 / 03 / 2003$

7T1 12:17

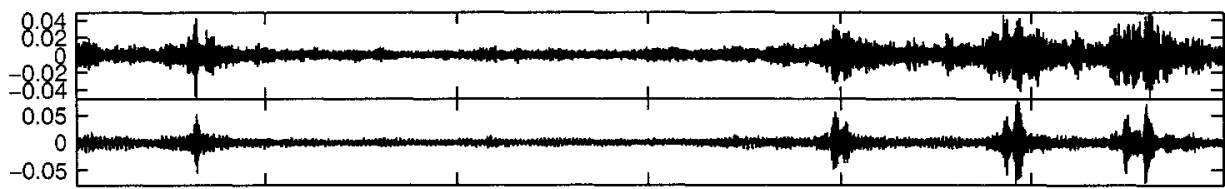

$7 \mathrm{~V} 1$

$14 / 03 / 2003$

$7 T 1$ 12:27

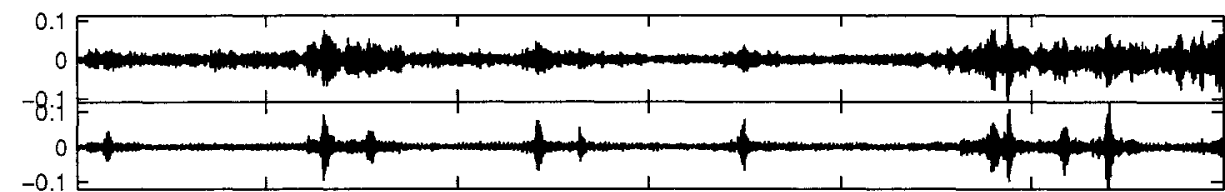

$7 \mathrm{~V} 1$

$14 / 03 / 2003$

$7 \Upsilon 11$ 12:37

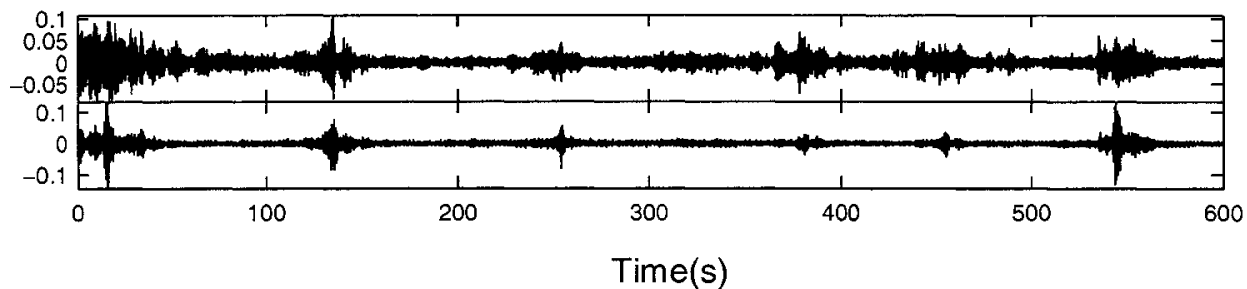

$7 \mathrm{~V} 1$

$14 / 03 / 2003$

$7 \mathrm{~T} 1 \mathrm{12:47}$

Figure 3-3: Vertical and lateral time histories at monitoring location 7 of baseline variability datasets. 

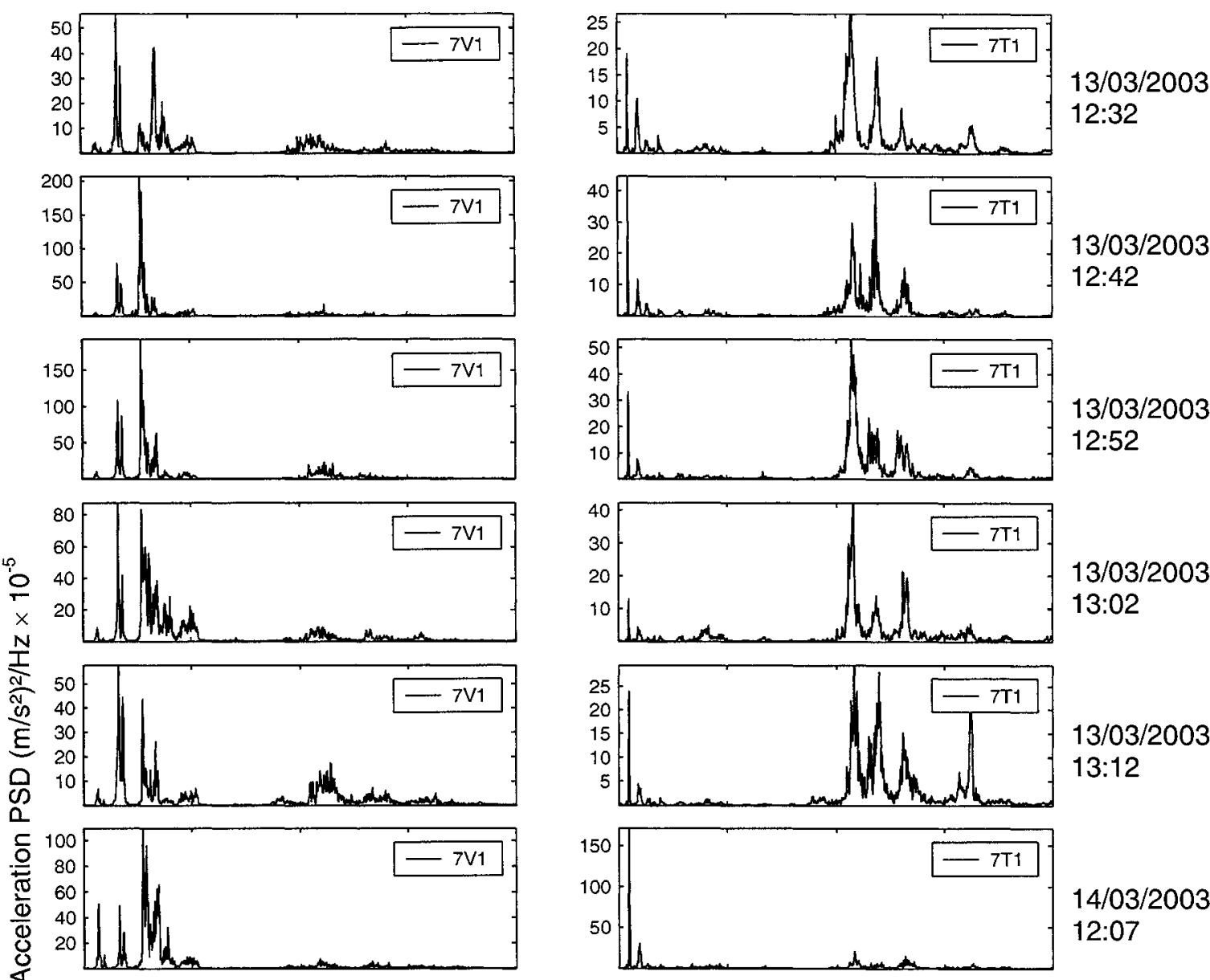

$14 / 03 / 2003$ $12: 07$
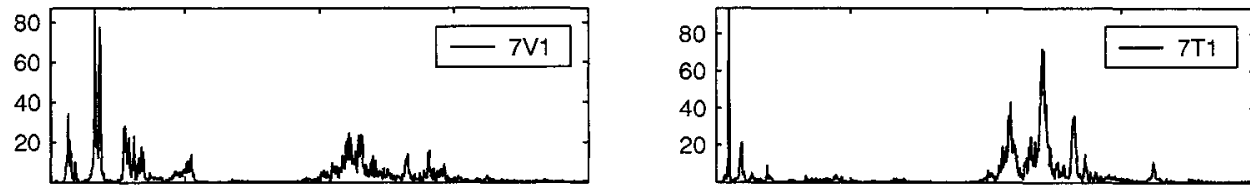

$14 / 03 / 2003$

$12: 17$
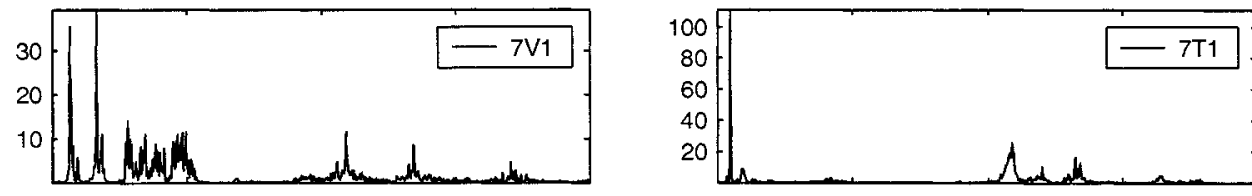

$14 / 03 / 2003$ $12: 27$
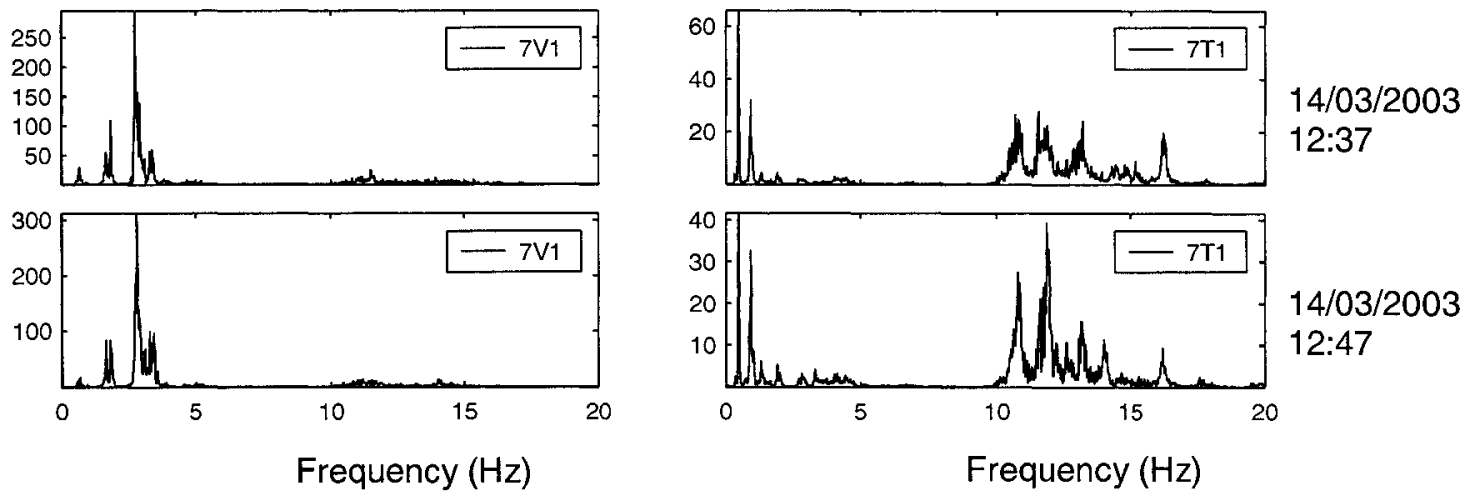

Figure 3-4: Frequency content of bridge responses at location 7 of baseline variability datasets. 


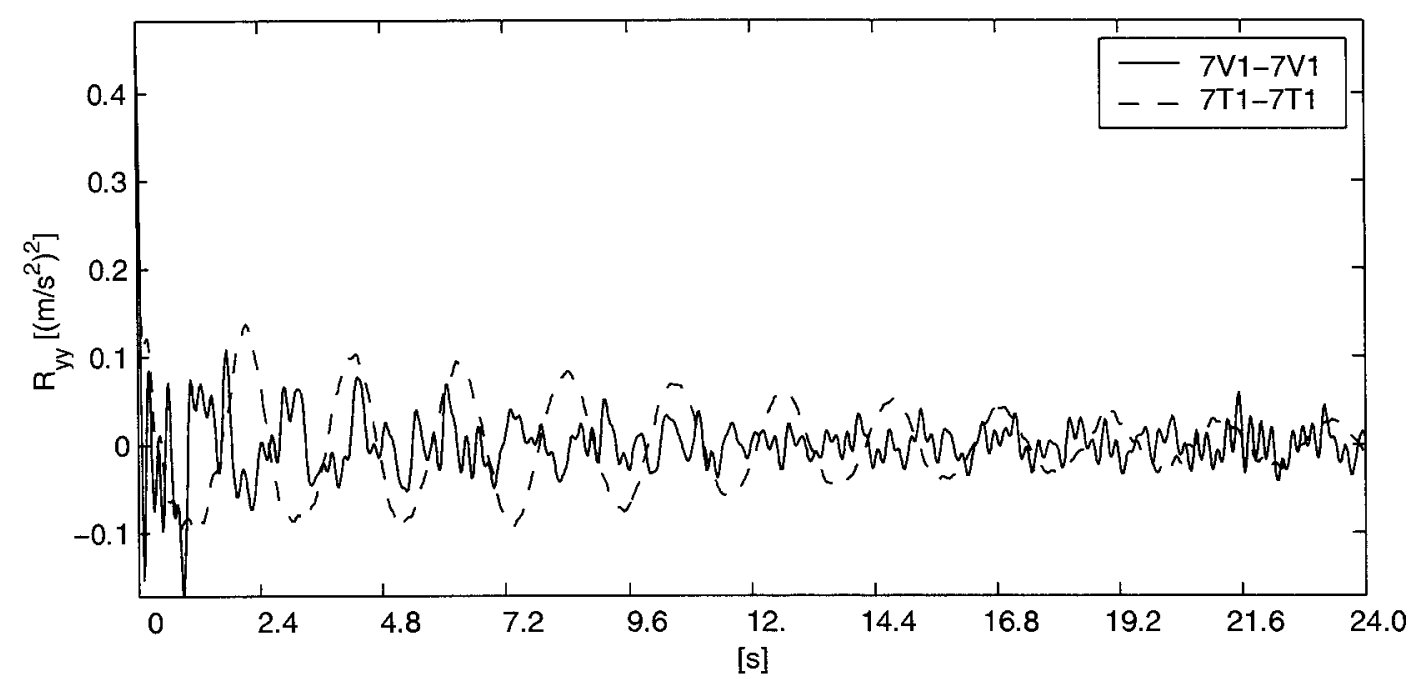

Figure 3-5: Typical data correlation estimates. 


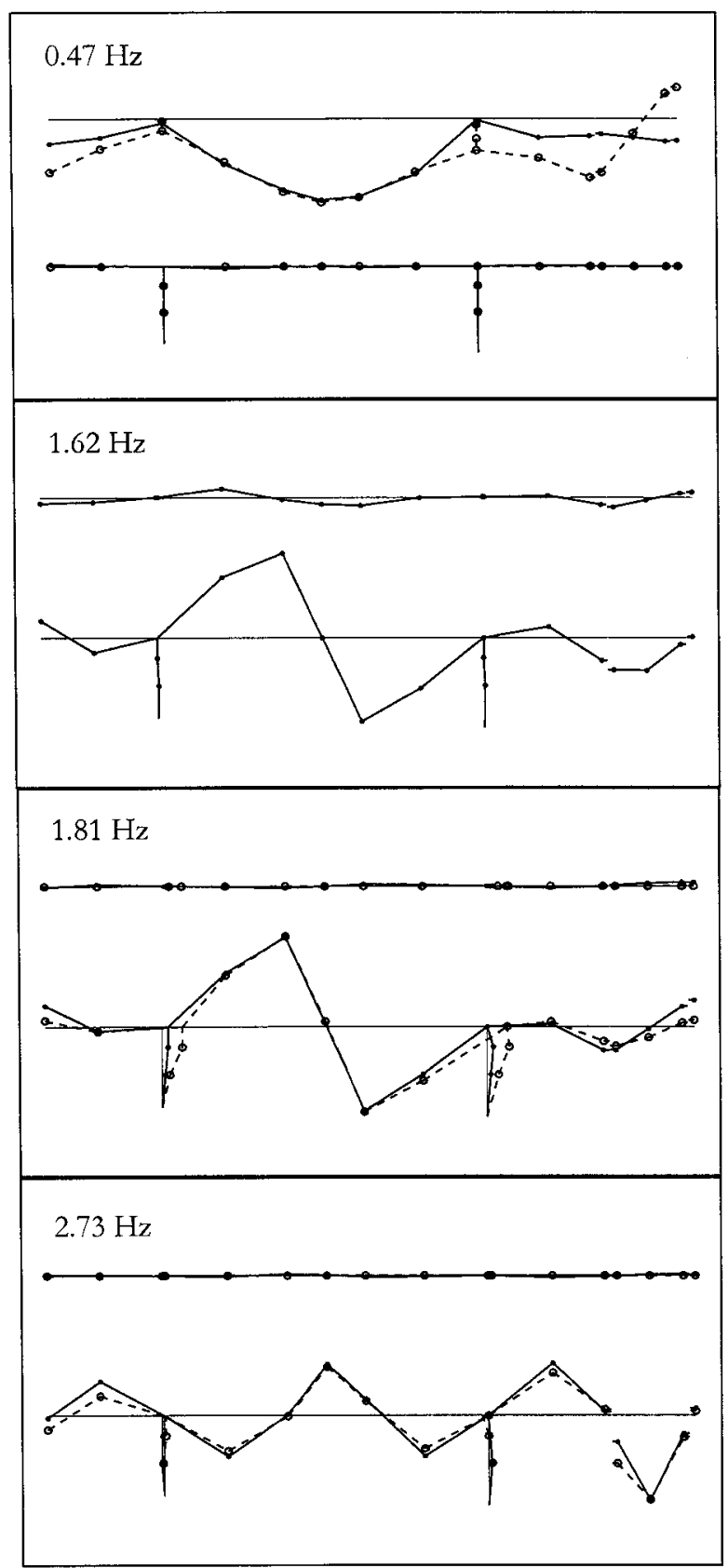

Figure 3-6: Vibration mode shapes. Full line: modes identified from the monitoring data; dotted line: modes from finite element model (not available for $1.62 \mathrm{~Hz}$ mode). 
Frequencies
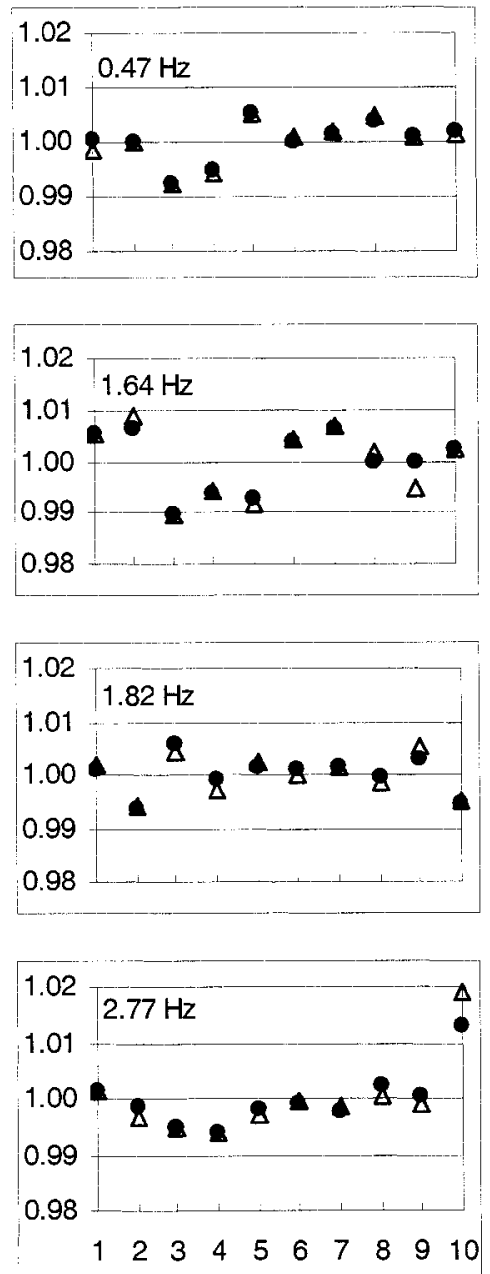

Dataset
Damping Ratios
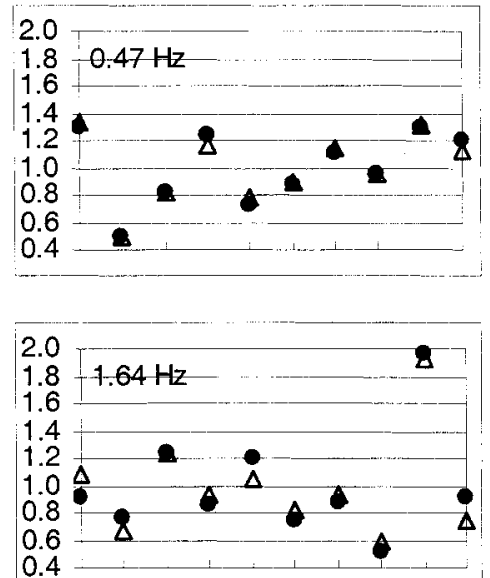

0.4
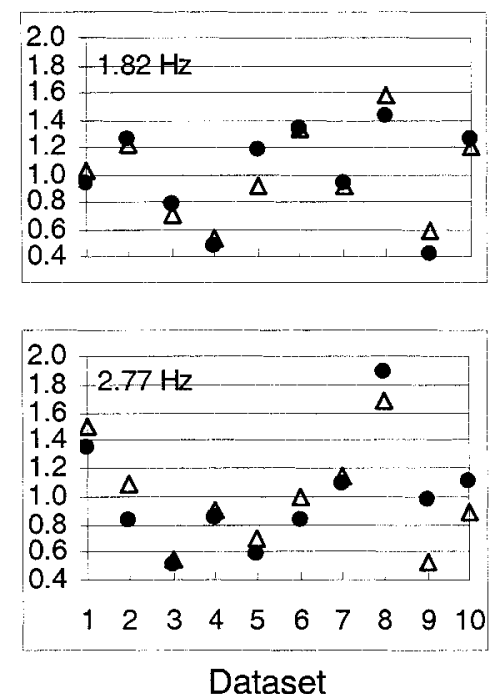

Mode Shapes (MAC)
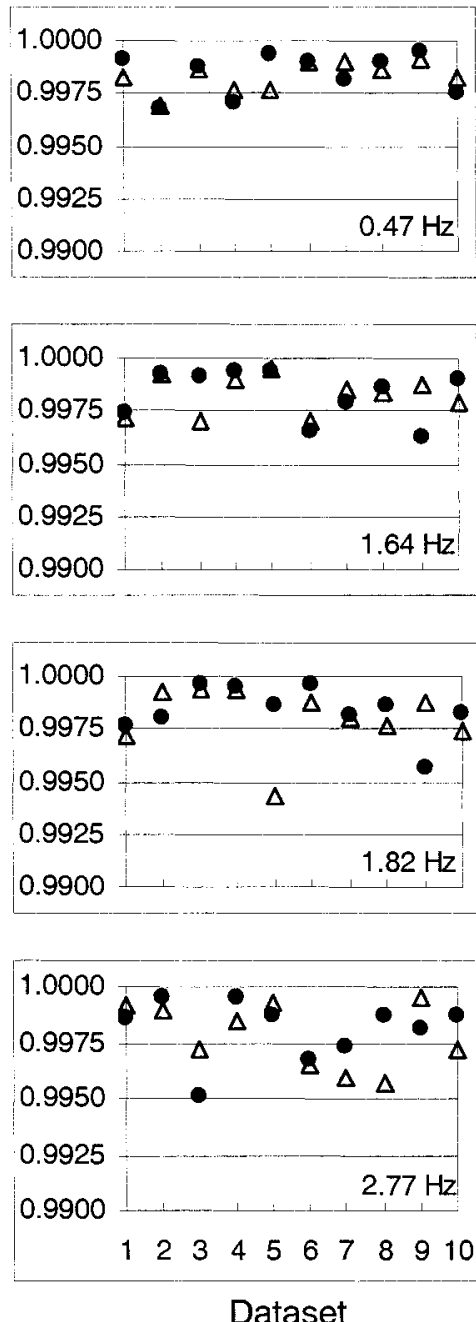

Figure 3-7: Baseline variability of extracted modal parameters. Eigenfrequencies and damping tatios are normalized by the mean values. Symbol "॰" is for lower order SSI solutions; " $\triangle$ " is for higher order solutions. 
Frequencies
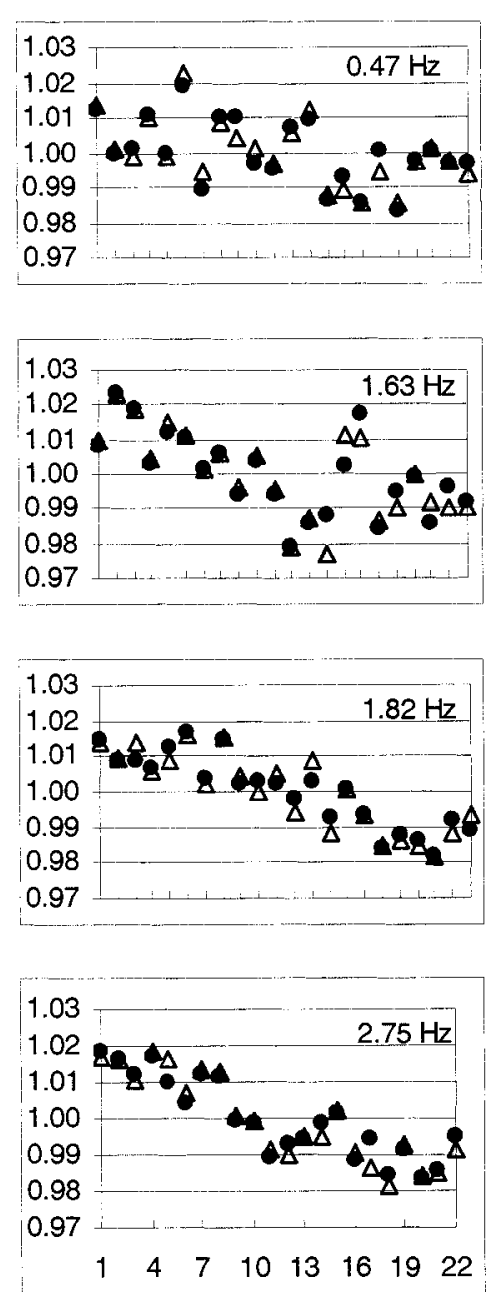

Dataset
Damping Ratios
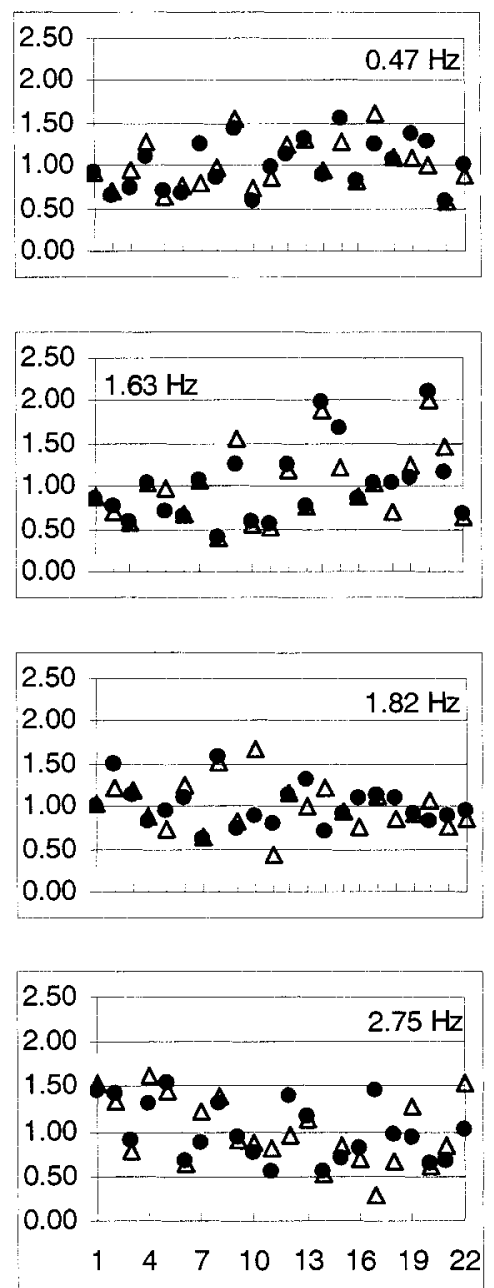

Dataset
Mode Shapes (MAC)
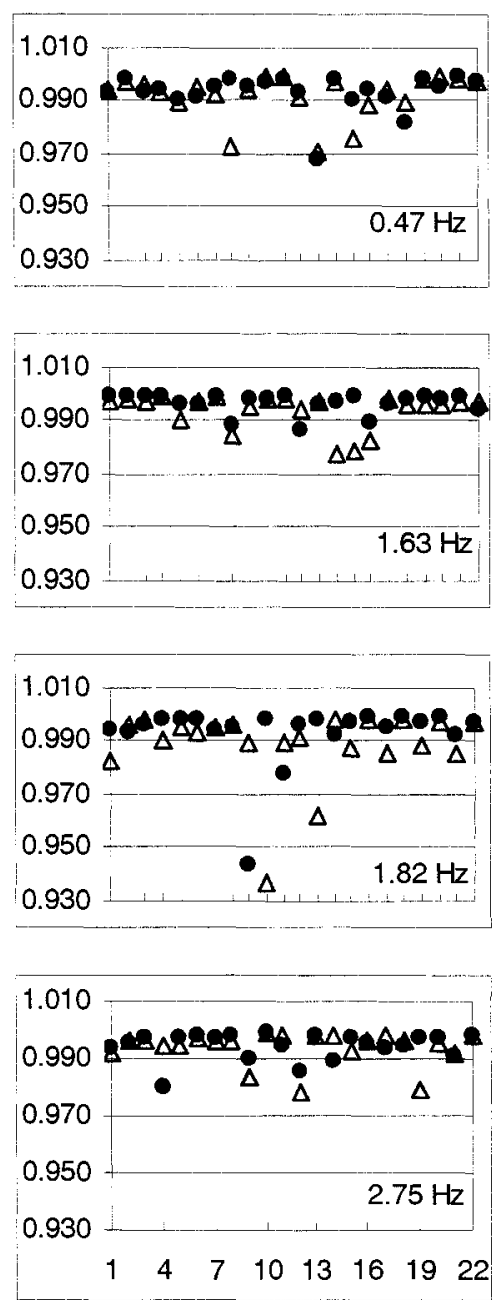

Dataset

Figure 3-8: General variability of extracted modal parameters. Eigenfrequencies and damping ratios are normalized by the mean values. Symbol "थ" is for lower order SSI solutions; " $\Delta$ " is for higher order solutions. 

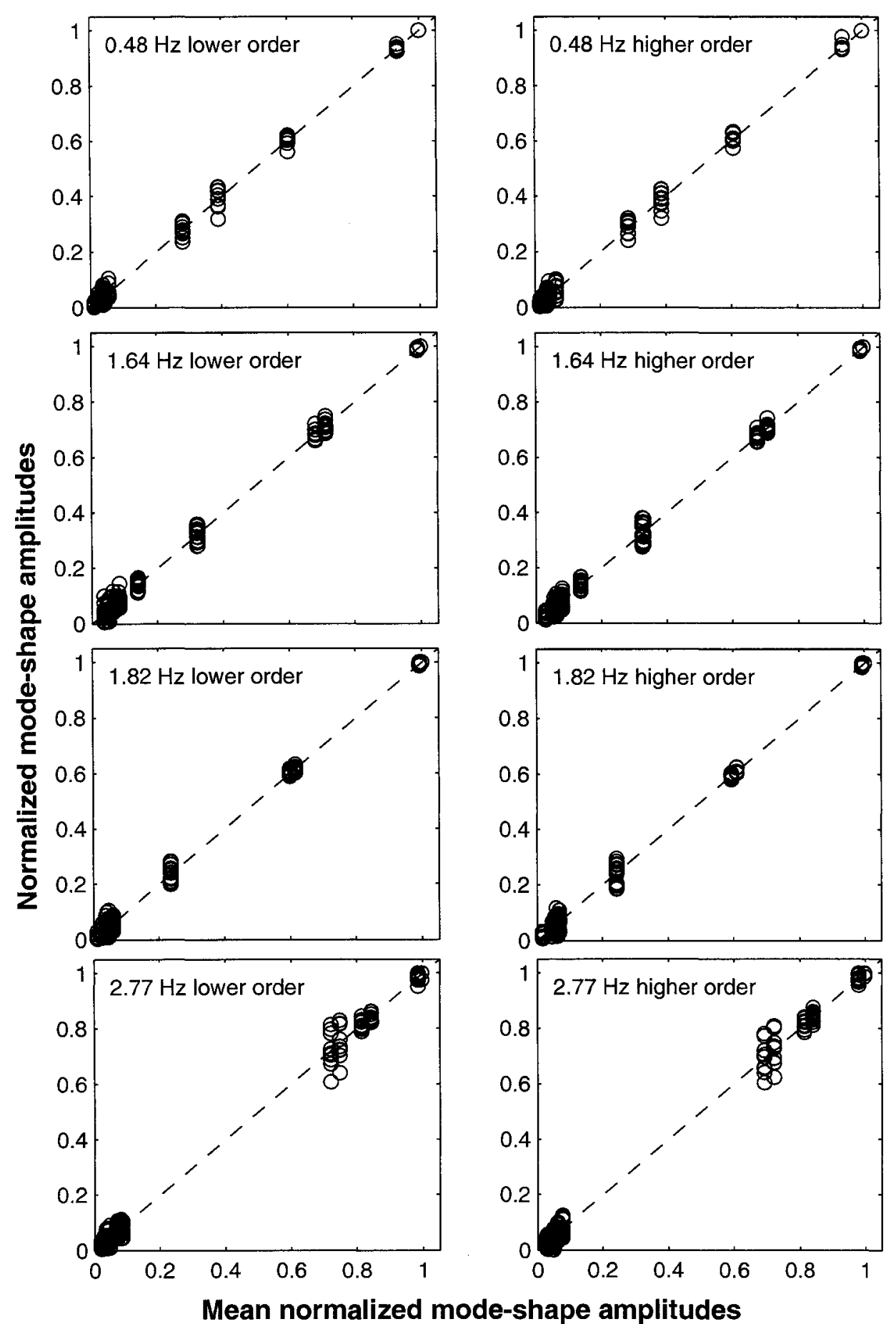

Figure 3-9: Baseline variability of identified mode shape coefficients under similar environmental and loading conditions, from analysis of 10 vibration datasets collected over a two-day period. 


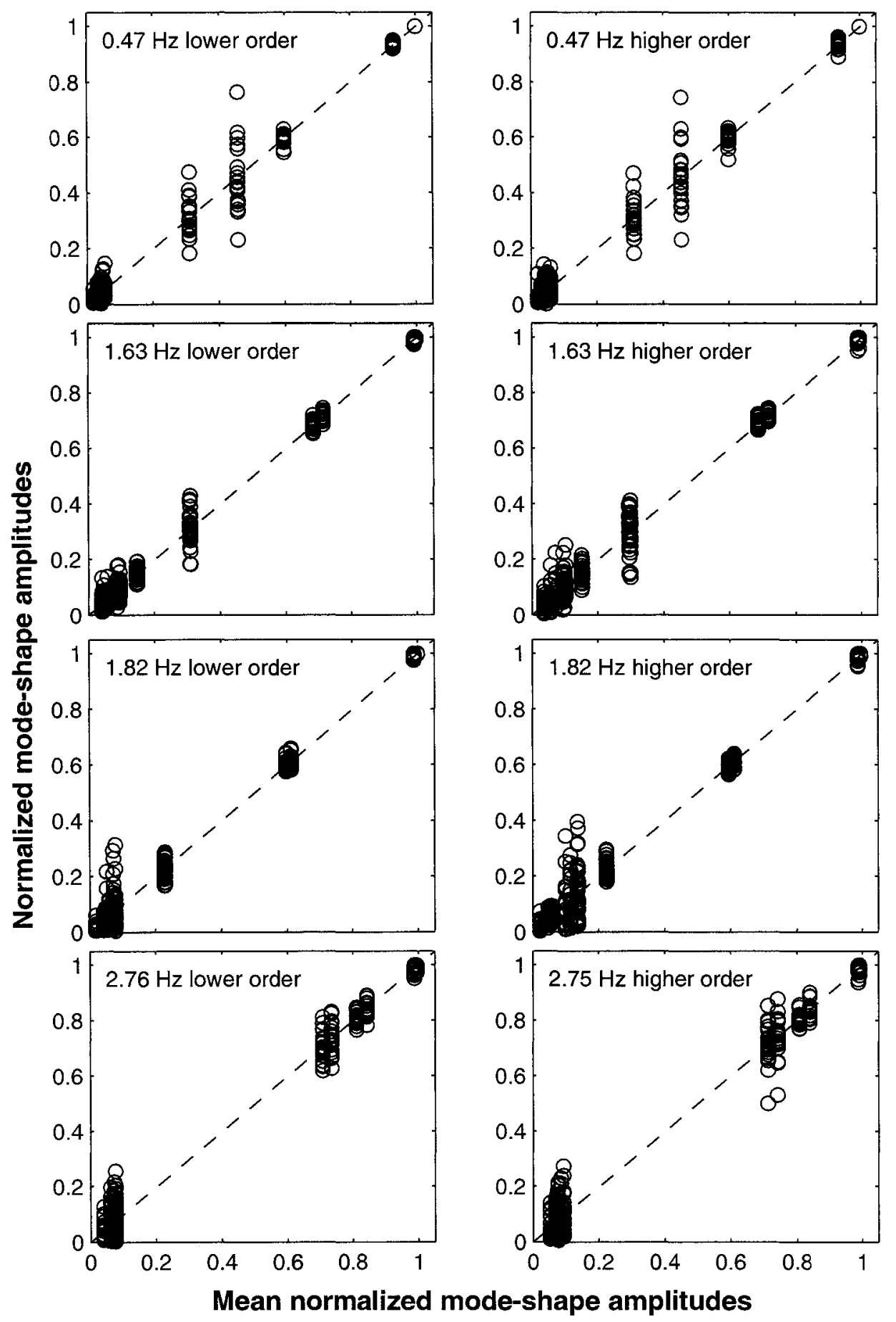

Figure 3-10: General variability of identified mode shape coefficients under operational conditions, from analysis of 22 ambient vibration datasets with fluctuating loading and environmental scenarios over six-month period. 

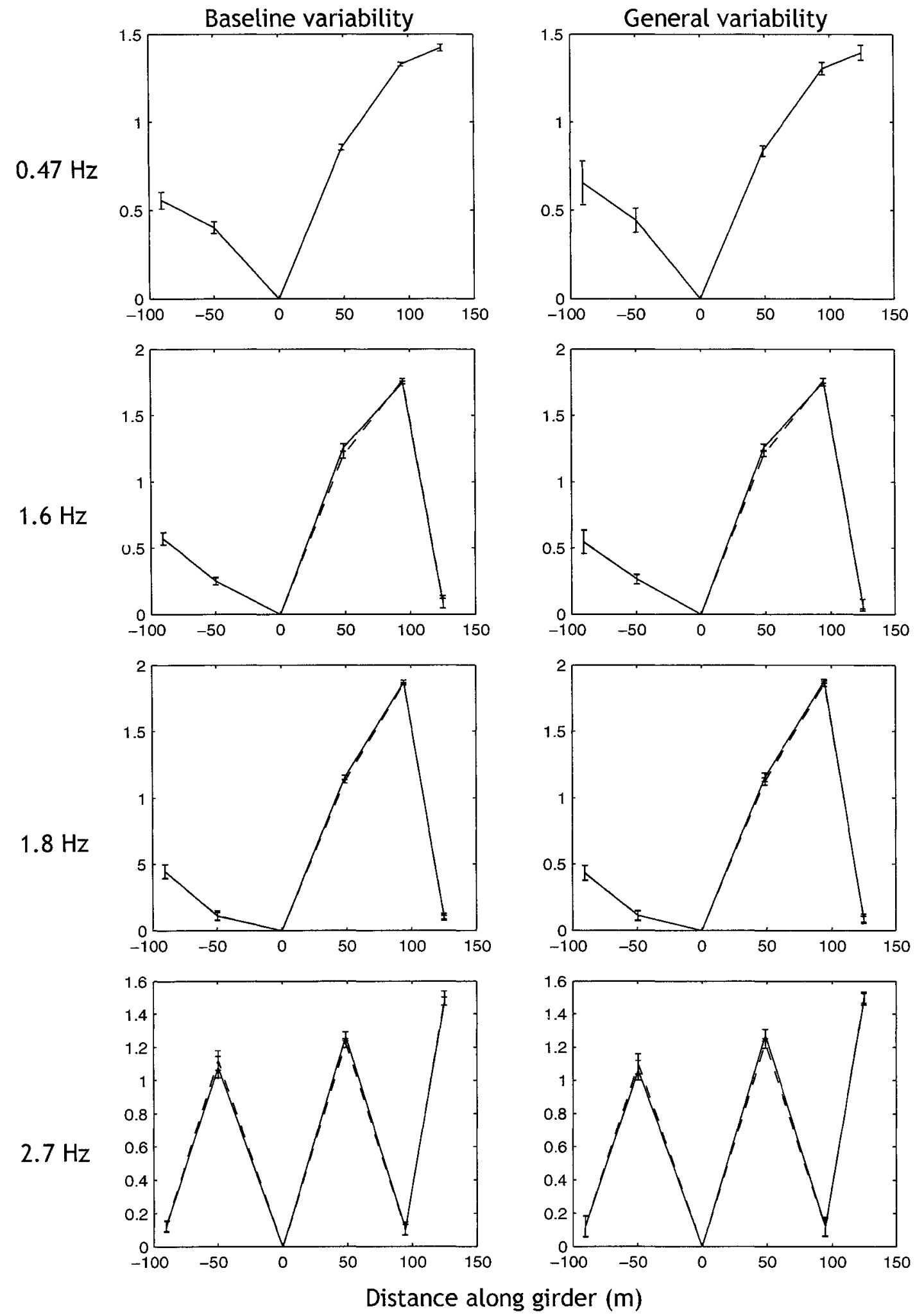

Figure 3-11: Variability of identified mode shapes. Average normalized mode shapes are shown with error bars indicating \pm one standard deviation. For vertical vibration modes (all except $0.47 \mathrm{~Hz}$ mode), full-line indicates north side of girder, dotted line: south side of girder 


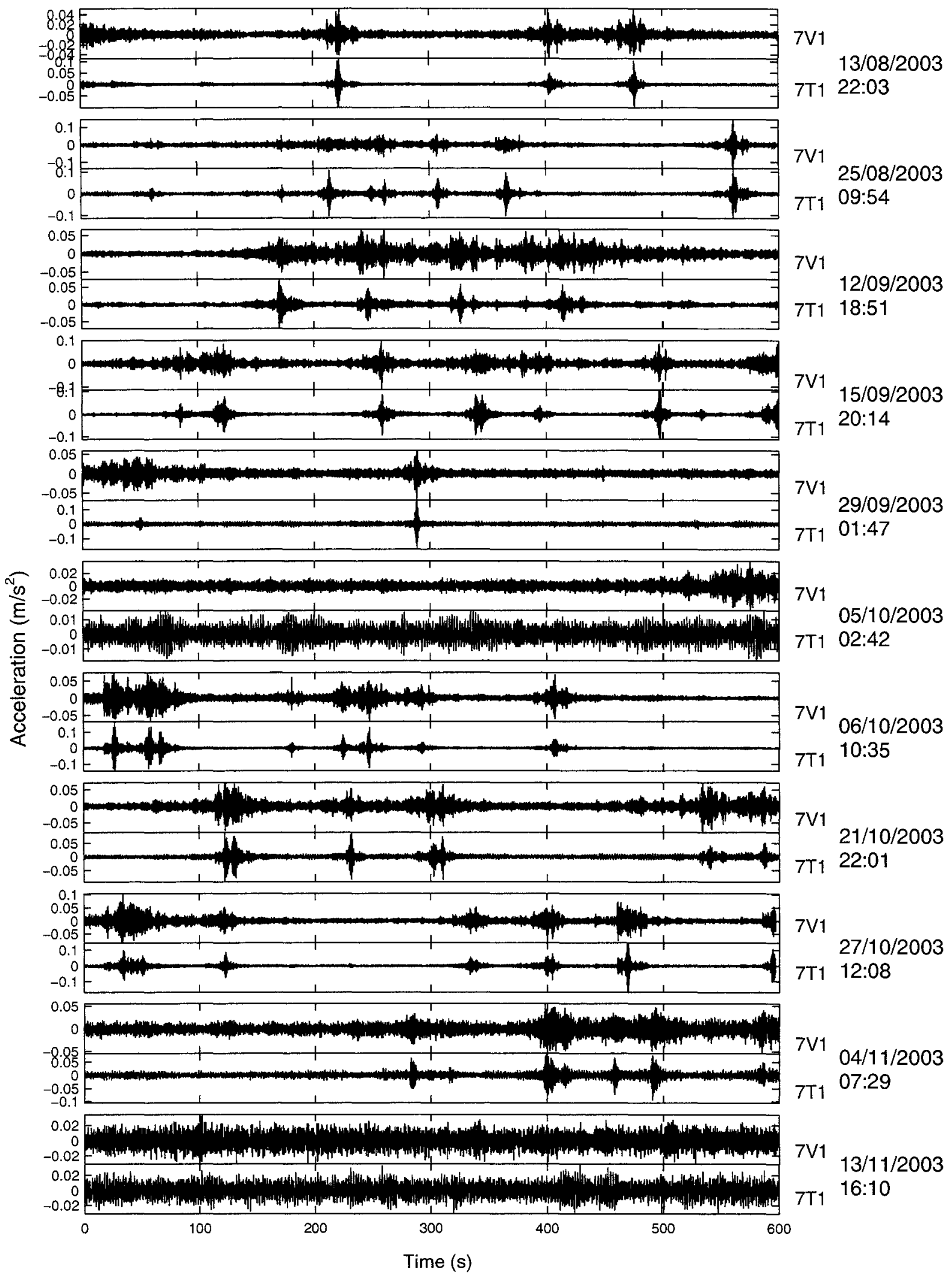

Figure 3-12(a): Vertical and lateral acceleration time histories at monitoring location 7 (datasets 1 to 11). 


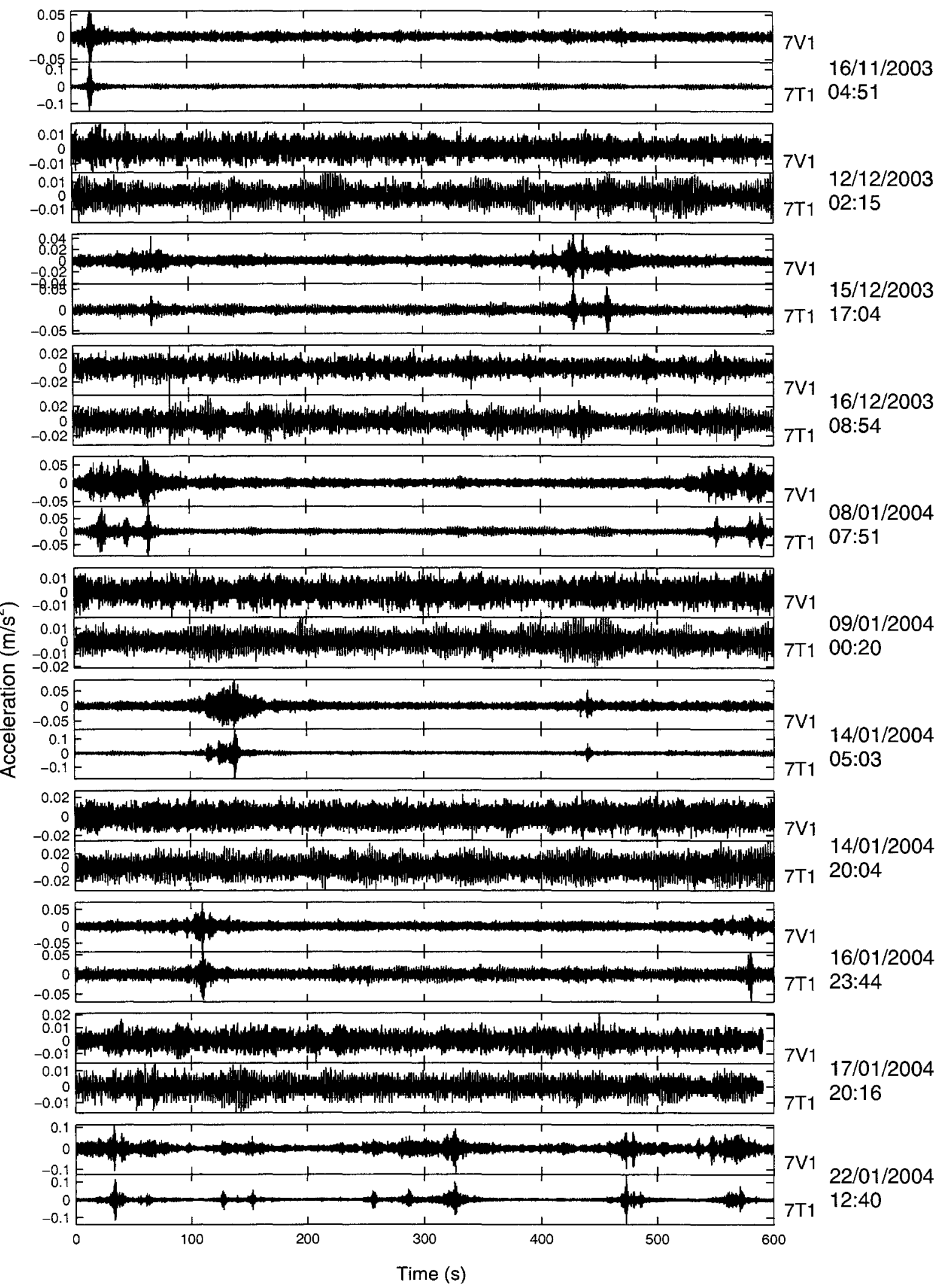

Figure 3-12(b): Vertical and lateral acceleration time histories at monitoring location 7 (datasets 12 to 22). 

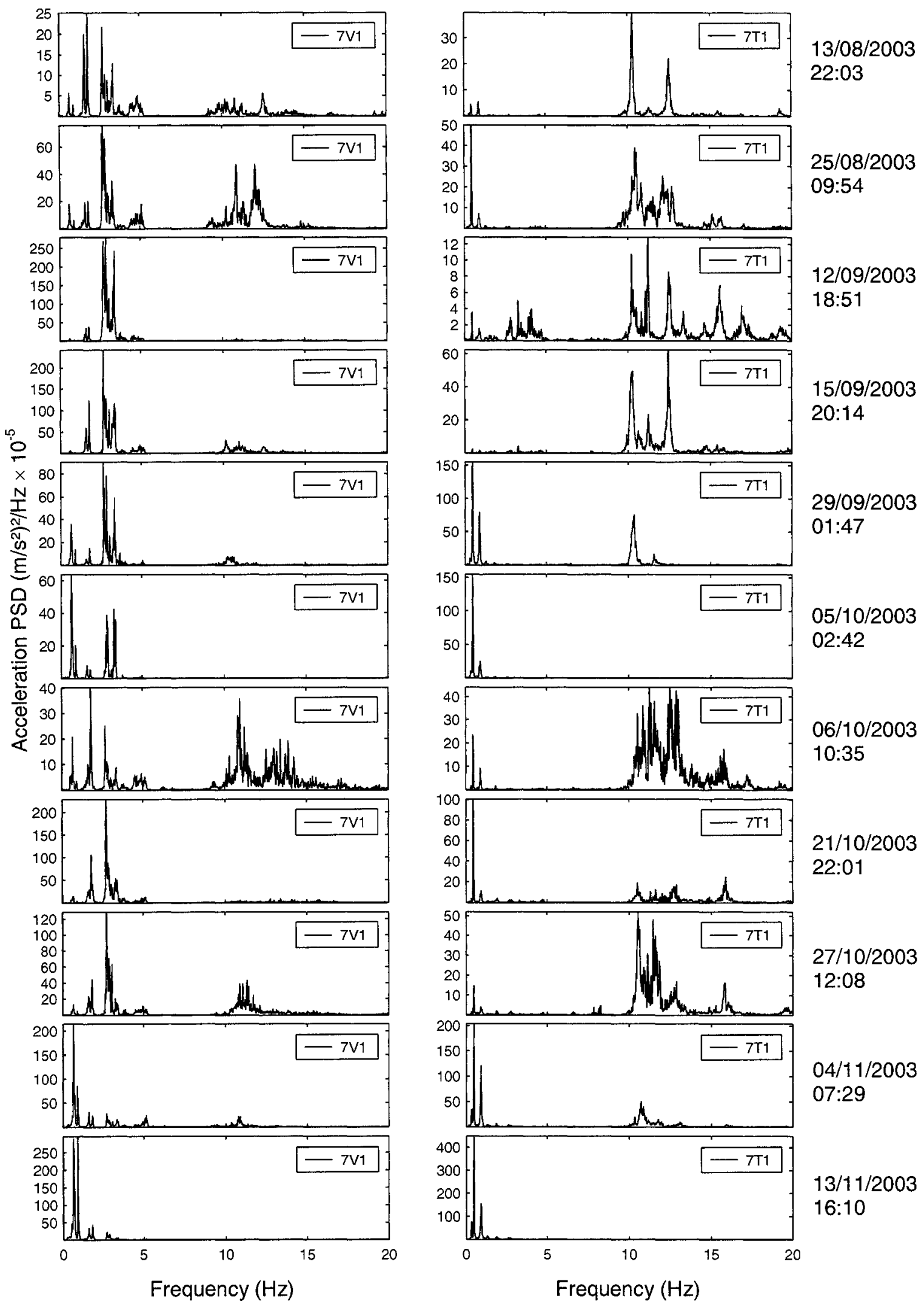

Figure 3-13(a): Frequency content of bridge responses at monitoring location 7 (datasets 1 to 11) 

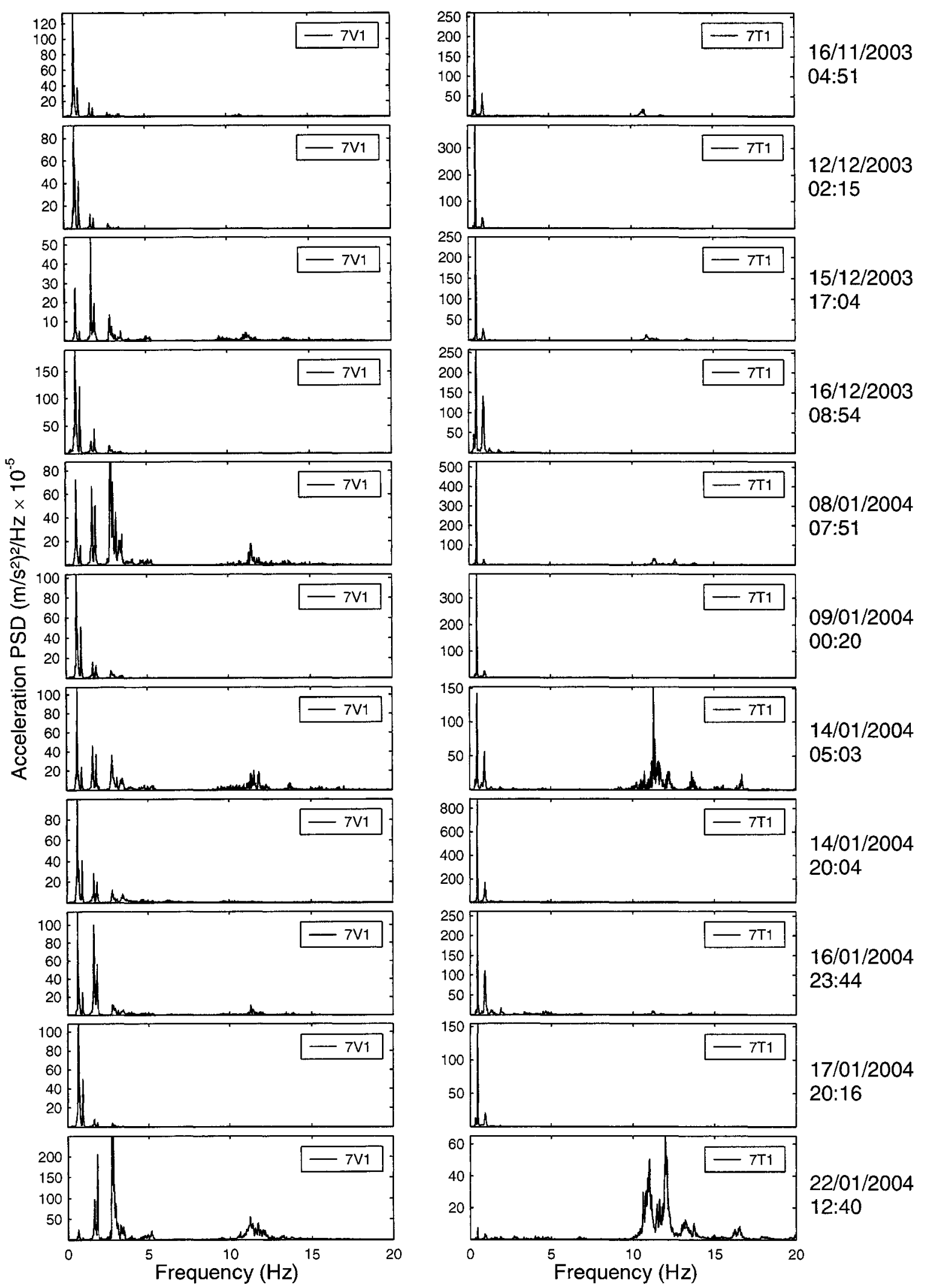

Figure 3-13(b): Frequency content of responses at monitoring location 7 (datasets 12 to 22) 


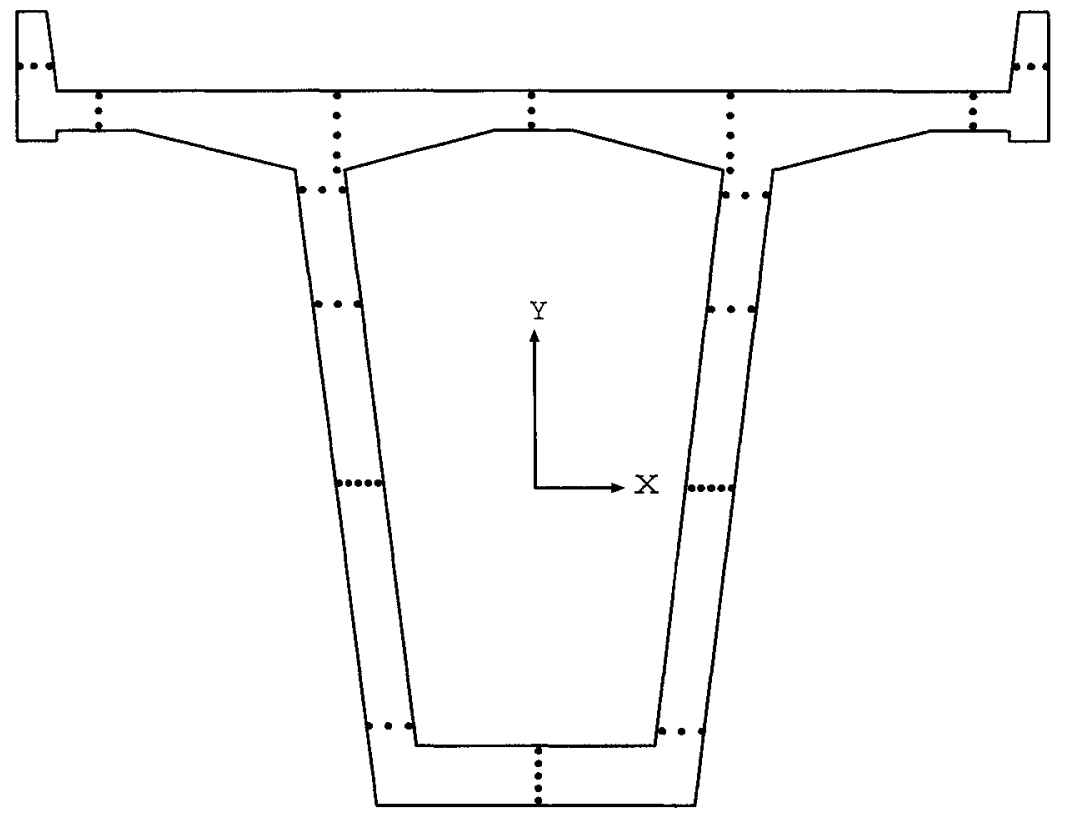

Figure 3-14: Typical thermocouple layout. 

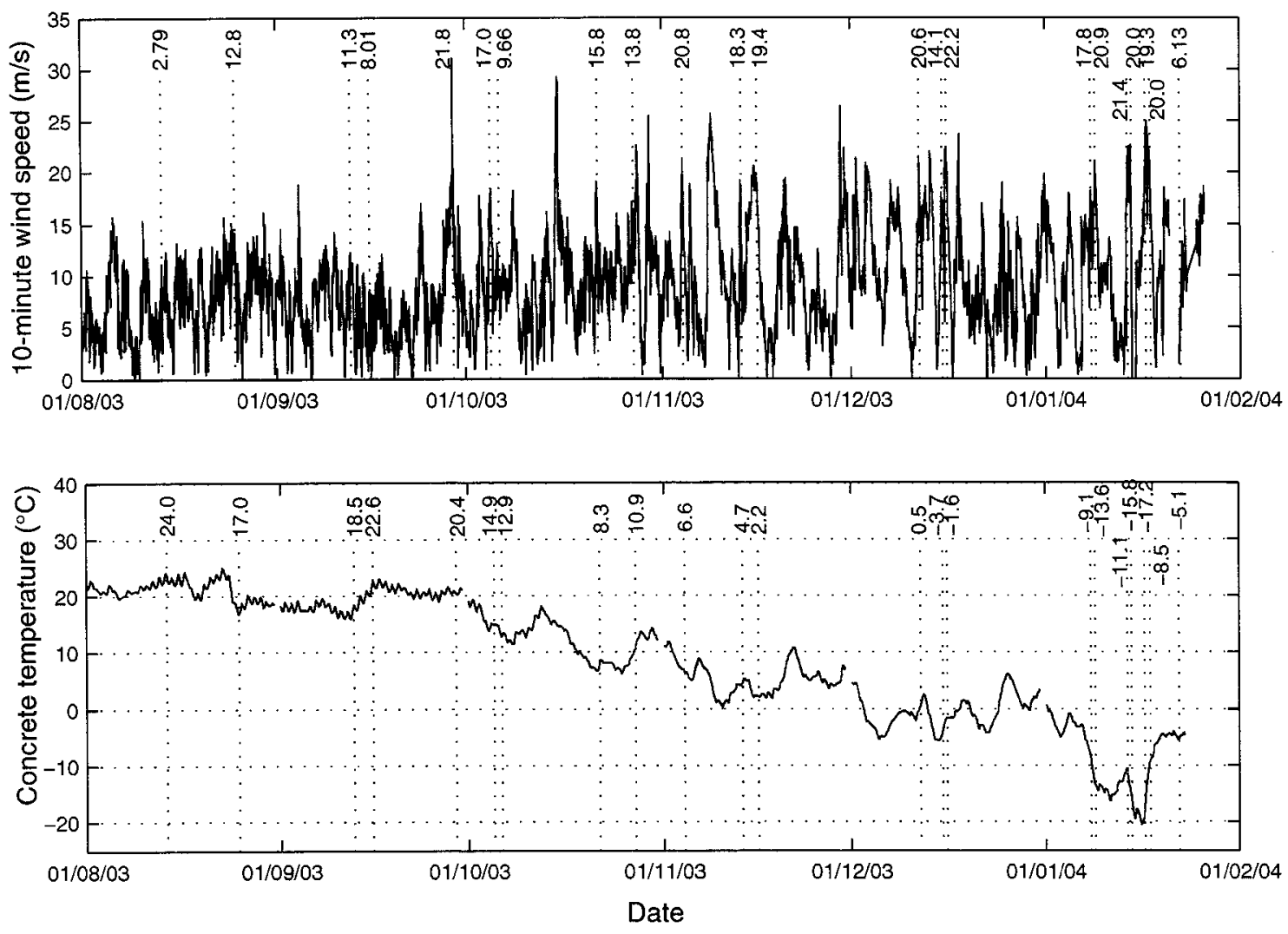

Figure 3-15: Wind speeds and average concrete temperatures at Confederation Bridge from August 2003 to mid January 2004. Values for the datasets of the variability study are indicated. 

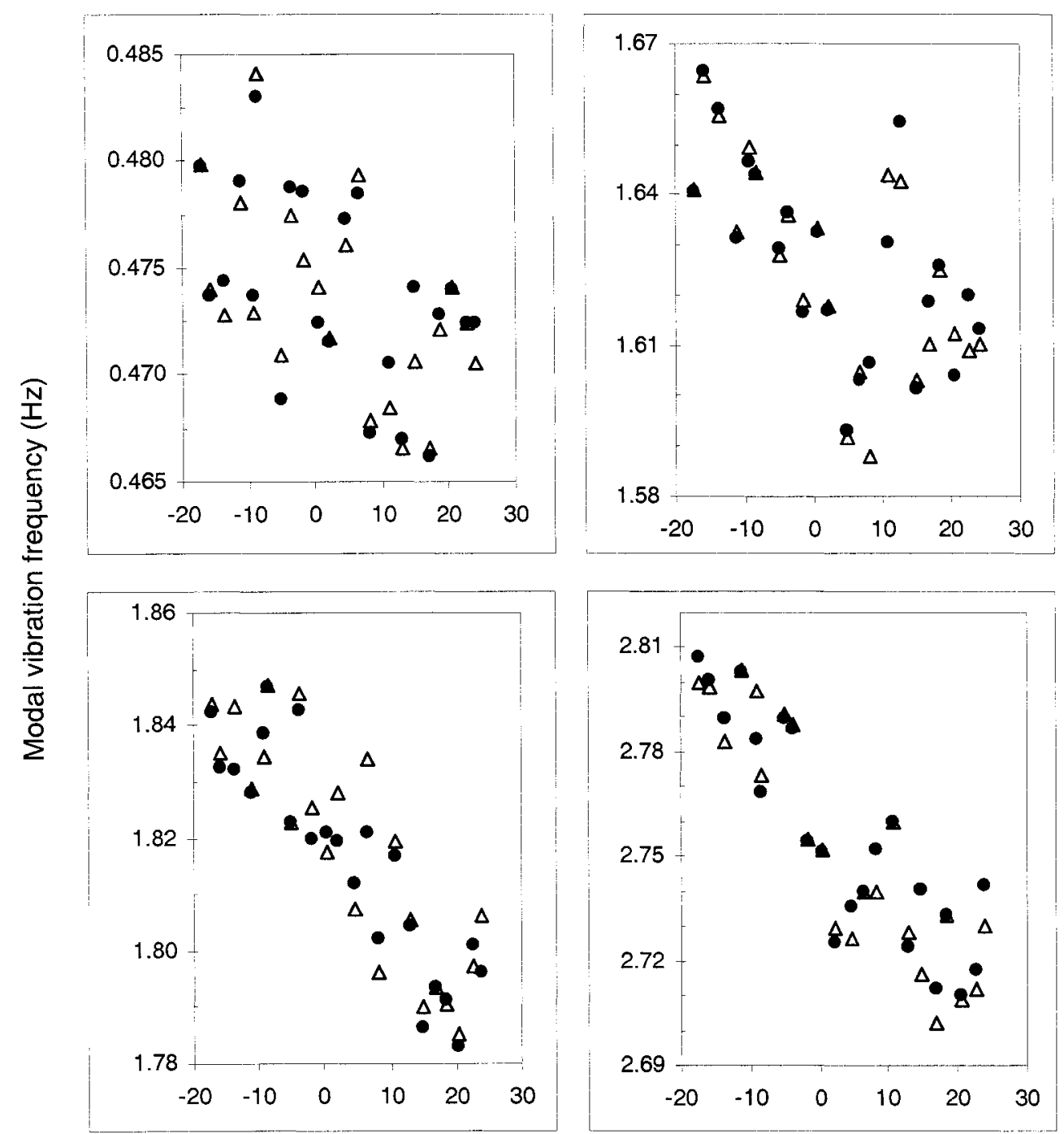

Average girder concrete temperature $\left({ }^{\circ} \mathrm{C}\right)$

Figure 3-16: Modal frequencies vs. average temperature of the concrete of the bridge girder. The symbols are: "•" for lower order SSI solutions; " $\triangle$ " for higher order SSI solutions. 


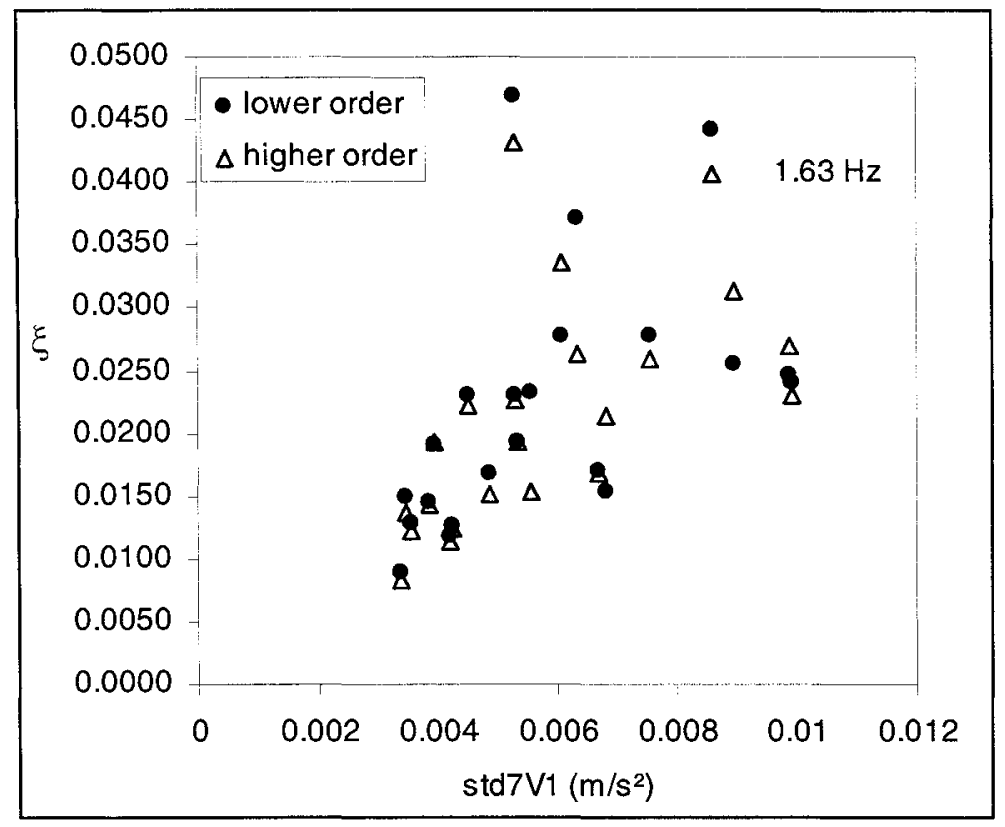

Figure 3-17: Identified damping ratios of $1.63 \mathrm{~Hz}$ mode against vibration amplitude standard deviations of reference channel $7 \mathrm{~V} 1$. 


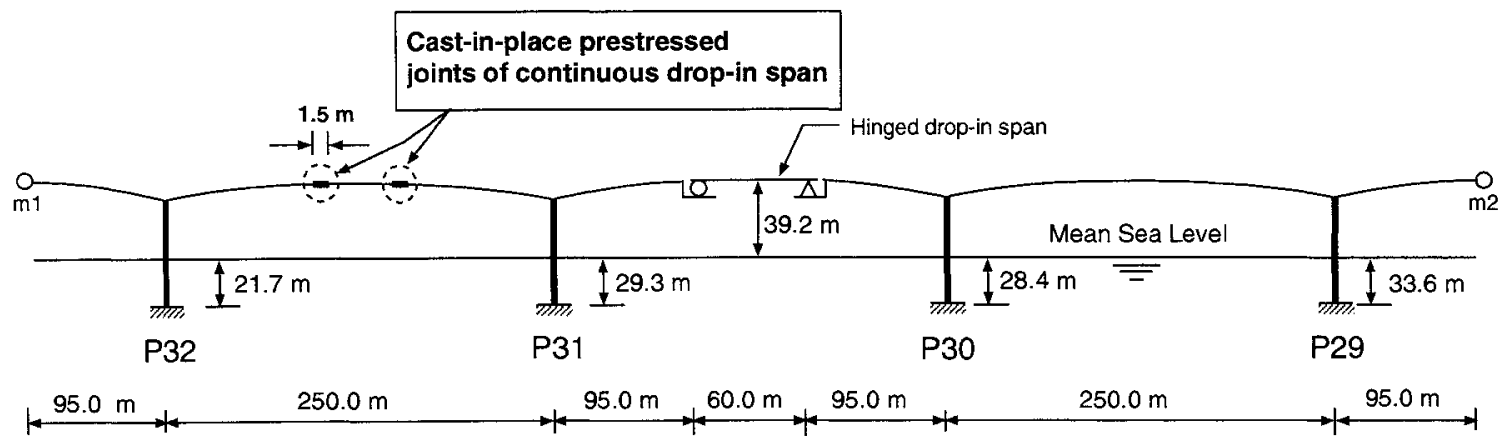

Figure 3-18: Locations of damage in simulated damage scenarios, shown in thicker lines. 

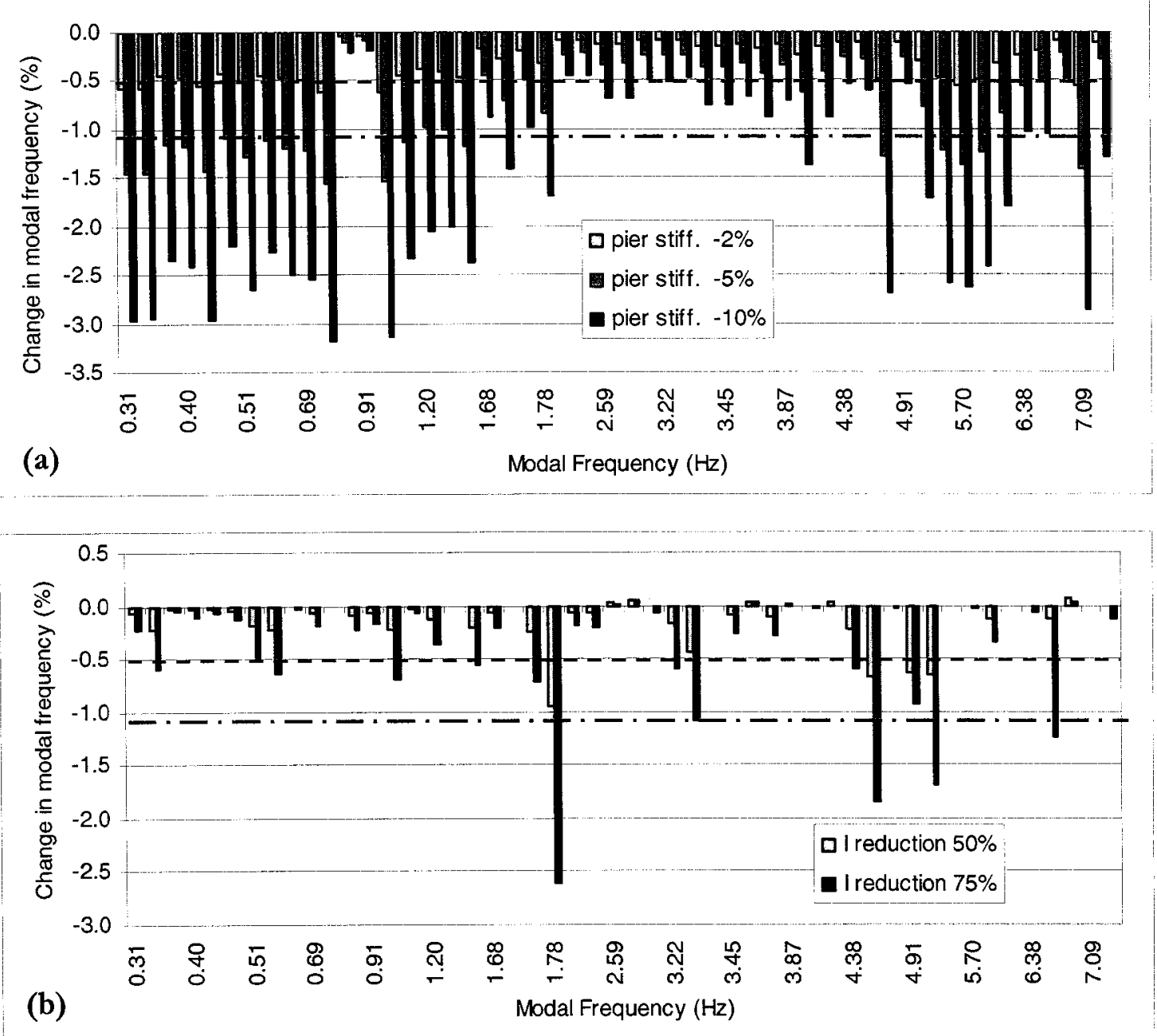

Figure 3-19: Sensitivity of modal frequencies to damage. (a) Pier deterioration scenarios, (b) Scenarios of damage at joints of continuous drop-in span. Dotted line: average baseline variability of modal frequencies; dash-dotted line: average variability under loading and environmental fluctuations. 
(a)

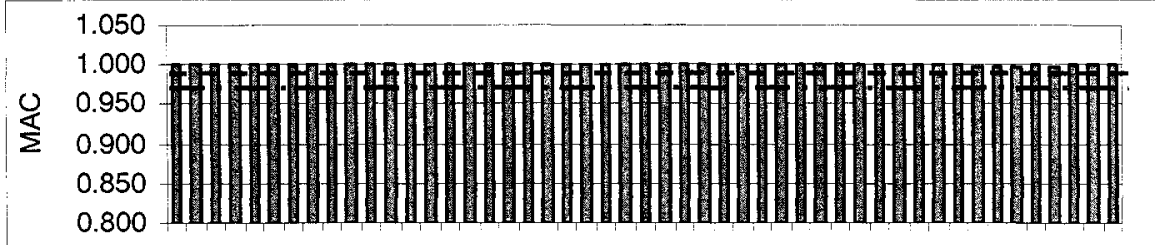

(b)

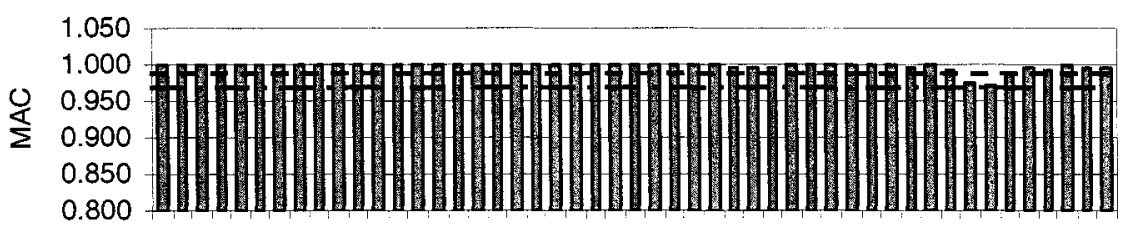

(c)

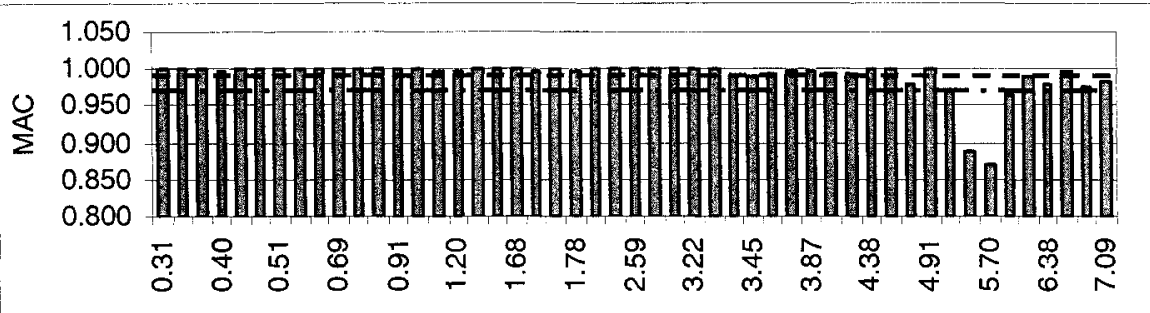

Modal frequency $(\mathrm{Hz})$

Figure 3-20: Sensitivity of mode shapes to damage scenarios of uniform pier deterioration with stiffness reductions of (a) $2 \%$, (b) $5 \%$ and (c) $10 \%$. Dotted line: average baseline variability MAC; dash-dotted line: average MAC under loading and environmental fluctuations. 
(a)

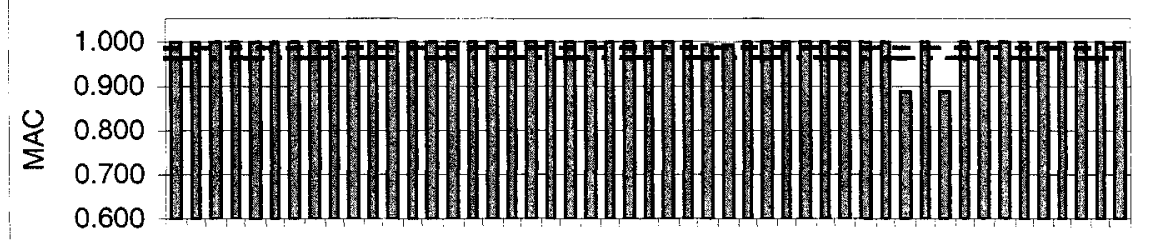

(b)

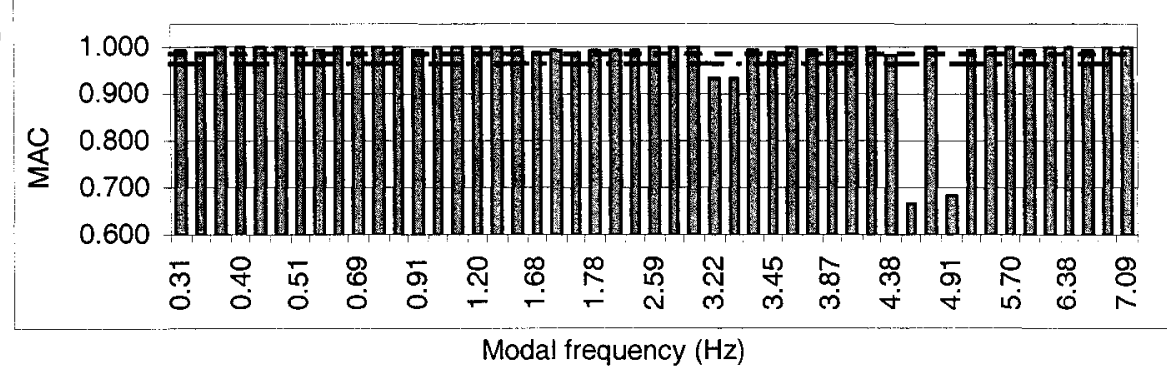

Figure 3-21: Sensitivity of mode shapes to damage at joints of continuous drop-in span with joint element inertia reductions of (a) $50 \%$ and (b) $75 \%$. Dotted line: average baseline variability MAC; dash-dotted line: average MAC under loading and environmental fluctuations. 

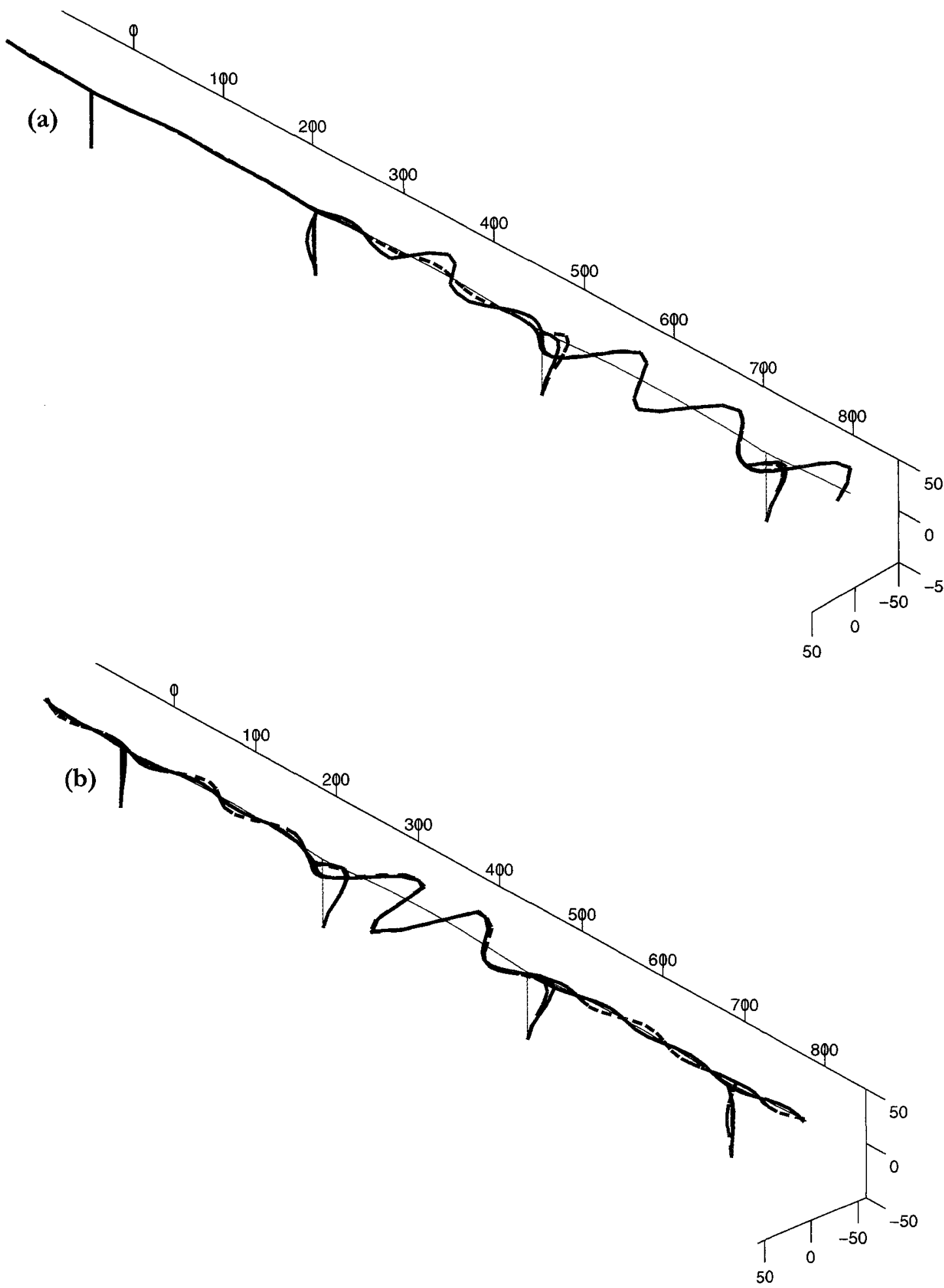

Figure 3-22: Mode shape changes after simulated $10 \%$ pier stiffness deterioration; (a) $5.57 \mathrm{~Hz}$ and (b) $5.70 \mathrm{~Hz}$. Full blue line: original mode shape; red dotted line: mode shape after damage. 

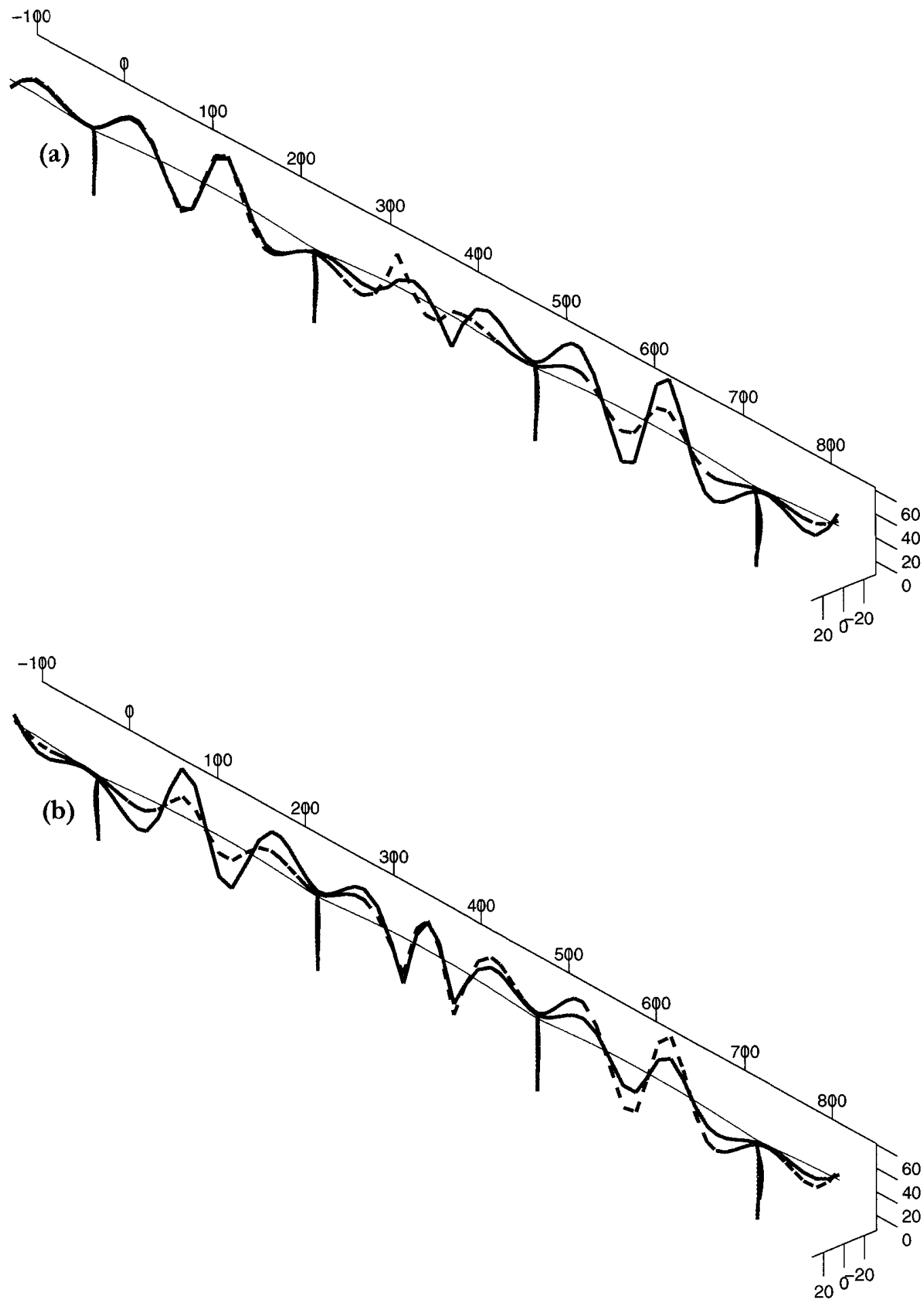

Figure 3-23: Mode shape changes after a simulated $75 \%$ stiffness reduction at the joints of a continuous drop-in span; (a) $4.81 \mathrm{~Hz}$ and (b) $4.91 \mathrm{~Hz}$. Full blue line: original mode shape; red dotted line: mode shape after damage. 
(a)

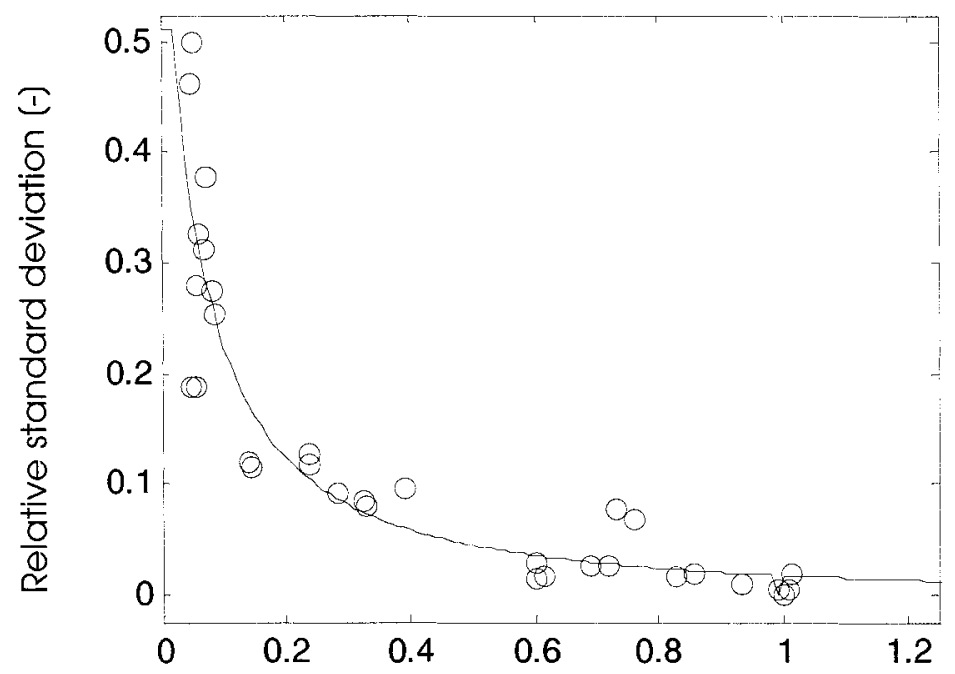

(b)

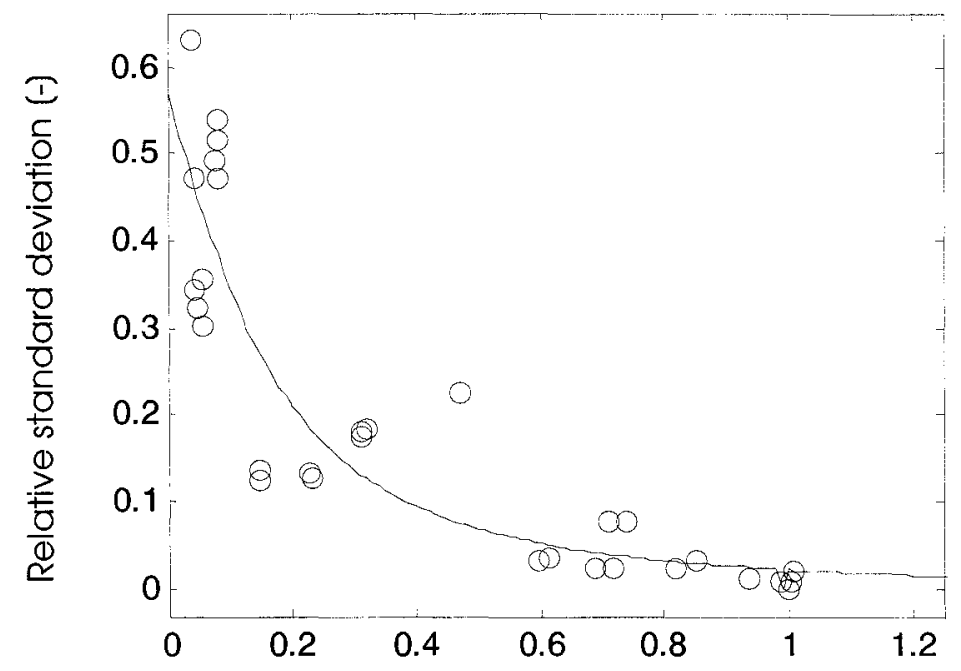

Modal amplitude normalized by amplitude of reference sensor location (-)

Figure 3-24: Variation of standard deviation of mode shape amplitudes with mode shape amplitudes extracted from (a) Baseline datasets, (b) General datasets. 


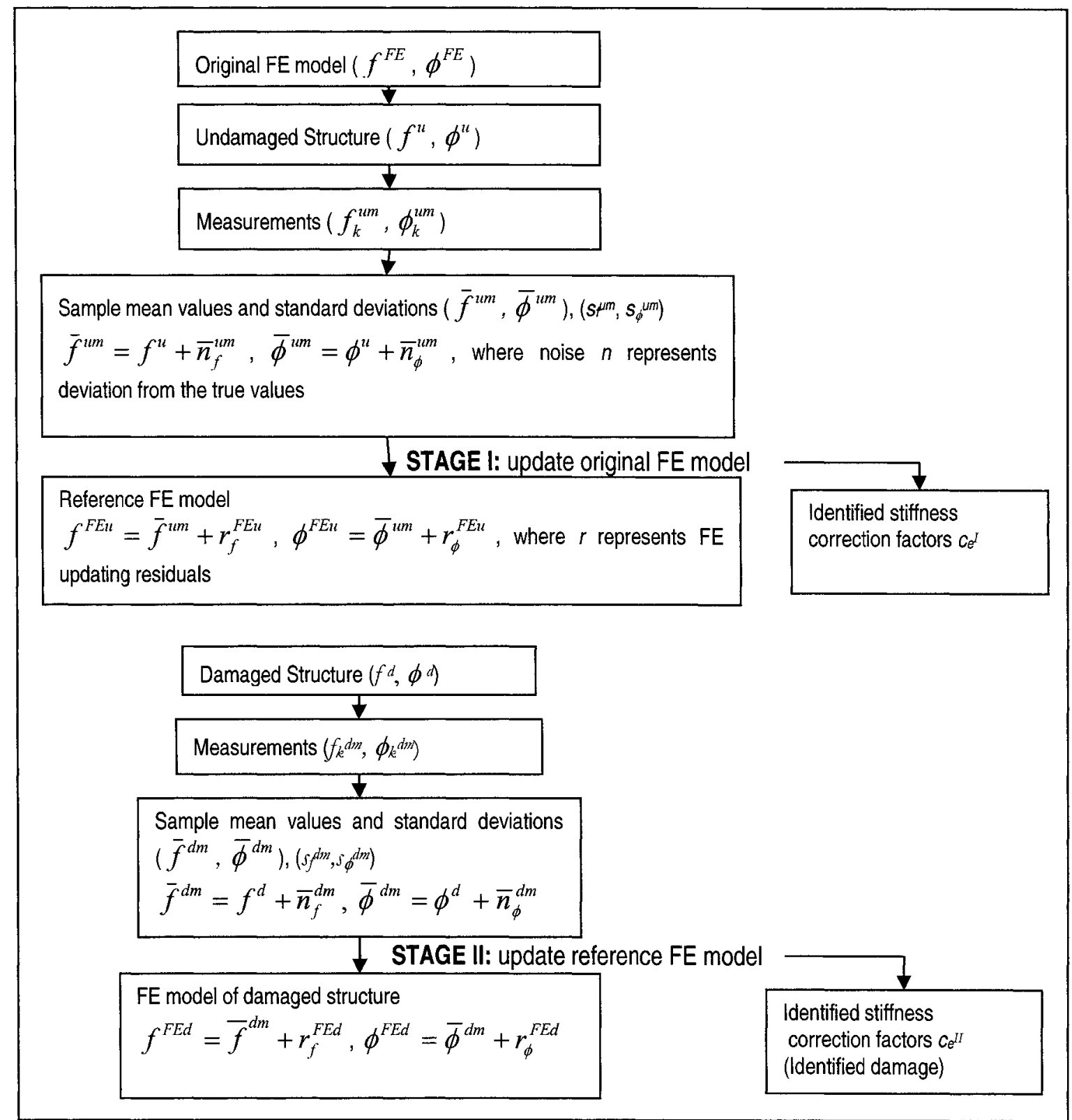

Figure 3-25: Schematic flow chart of a finite element updating damage detection process 


\section{Chapter 4. Application platform for processing, analysis and visualization of Confederation Bridge monitoring data}

\subsection{Introduction}

Numerous structural monitoring systems have been developed and installed in the field to collect information on the performance and behaviour of civil engineering structures and systems, such as buildings and bridges. The processing and analysis of large datasets collected from continuous monitoring systems often require a significant amount of time and effort. In order to accelerate the processing of these continuous monitoring data and to facilitate more rapid data analysis, and more timely interpretation and use of the results, a real-time data processing and analysis application platform has been developed which encompasses all aspects of data manipulation. This application platform consists of data processing, analysis and visualization modules, all integrated through graphical user interfaces (GUIs). The applications are designed and adapted to run in a real-time mode by automatically sorting incoming data and re-directing it to the processing and animation modules for graphic display of bridge displacements and motion in near real-time, as limited by the network speed. With this capability, after the occurrence of extreme events such as windstorms, earthquakes or ship impacts, bridge responses and condition of the facility can be assessed in a timely manner for decision support of its operation. The research opportunities that can be explored using the computer tool applications presented in this chapter are illustrated by a discussion of recent research results.

Because of the aging problems of transportation structures in Canada and many other countries in the world, owners of bridges, such as ministries of transportation and municipalities, and other various levels of government, have to devote increasingly larger 
proportions of their budgetary resources to maintenance spending to repair, retrofit, rehabilitate or replace deteriorated or damaged infrastructure for delivery of adequate service to the public. The total figure of infrastructure maintenance cost in Canada is estimated to be around $\$ 6$ billion Canadian per annum (CPWA 2003). These maintenance costs are on a rising trend due to the fact that a good portion of the existing infrastructure stock is fast reaching the end of their design service life. Currently, owners and operators of bridges base their infrastructure asset management decisions largely on information gathered through visual inspections, which may be aided by some localized diagnostic techniques such as acoustic, ultrasonic and magnetic field non-destructive testing methodologies. However, the visual inspection approach even when aided by the mentioned techniques is known to have limitations such as inaccessibility to some parts of the structure, inability to detect hidden internal damage, the need of a priori knowledge of damage location, and that it can only be carried out at periodic intervals, which together limit its ability to detect early signs of deterioration, which is necessary to devise cost effective counter-measures.

With the advent of information technology (IT) and the recent and ongoing developments in vibration based structural health monitoring an alternative approach to obtain timely information about the condition of a structure has become feasible in engineering practice. The vibration based techniques can overcome many limitations of previous diagnostic techniques. Application of the vibration based structural evaluation techniques will lead to better informed decision making and better prioritization in the allocation of limited resources for more cost effective bridge maintenance and asset management.

Vibration based structural condition assessment or structural health monitoring is a relatively new field of research in civil engineering that has seen rapid developments in recent 
years. Numerous monitoring systems have been developed and installed in the field to collect information on the material properties and structural behaviour of civil engineering structures and systems, such as buildings and bridges (e.g. Cheung et al. 1997; Koh et al. 2003; Katsuchi et al. 2003; Abe et al. 2000). The challenges and research needs of vibration based structural monitoring are recognized and much research has been carried out to address the problems, such as the development of special numerical system identification techniques for accurate determination of dynamic properties from output-only vibration measurement data of which the sources of excitation are not known or measured (Peeters and De Roeck 1999). To have a more effective impact on reducing the cost of the aging infrastructure problem, the development in the various relevant areas of structural health monitoring of data acquisition, system identification methodologies and damage detection algorithms should be complemented with parallel development in practical and user friendly computer application tools which will facilitate field application and use of the techniques by infrastructure operators and owners. The primary purpose of these computer application tools from the user perspective is to simplify and facilitate the process of extracting meaningful engineering information from the monitoring data. Efficient computer user interfaces are also essential components in a continuous monitoring system to provide rapid and accurate condition assessment of the monitored structure either on a continuous basis or after the occurrence of an extreme event, such as an accidental overload, an earthquake, flood, hurricane, impact from traffic or shipping accident or explosion. The research presents numerous challenges on the development of intelligent automatic data processing and analysis, reliable condition assessment algorithms that take into account the stochastic variations in data behaviour, and the development of graphical user interfaces (GUIs) and visualization tools to facilitate engineering interpretation of the monitoring information. 
The Confederation Bridge Monitoring Project in Canada presents a unique opportunity to address and explore these challenges. The $12.9 \mathrm{~km}$ Confederation Bridge crosses the Northumberland Strait in Eastern Canada, linking the provinces of NewBrunswick and Prince Edward Island. It is the world's longest bridge constructed over ice covered water and one of the longest continuous multi-span bridges. The structural dynamics monitoring system of the Confederation Bridge, in operation since 1997, consists of a sophisticated network of sensor instrumentation and multiple continuous data acquisition systems to capture the dynamic responses of the bridge under wind, traffic, ice floes and earthquake loads. The monitoring system is designed with advanced automatic data collection algorithms to record bridge responses under both normal ambient vibration conditions on a continuous basis or triggered under extreme loading scenarios.

A prototype monitoring application platform developed for the Confederation Bridge, which has been recently described by Desjardins et al. (2006), is presented in this chapter. The application platform has been developed to facilitate the processing, analysis and interpretation of the large datasets collected from the continuous monitoring. It covers all aspects of data manipulation from data management, processing and analysis to display and visualization of the data and analysis results for potential use of the information for decision support in the operation and management of the monitored structure. The application tools and interfaces greatly reduce the amount of time and effort needed to obtain numerical results and observation findings from the monitoring data.

The developed application platform has a modular design in which each module has its own GUI for configuration of the processing of data and is designed to interface seamlessly with other appropriate modules in the application platform. Modules for data processing, data display and plotting, system identification, and visualization of bridge 
responses have been developed and their design details are presented in this chapter. With the modular design, additional application modules can be developed and added to the platform to perform new tasks in the future. Another advantage of the modular design is that the application architecture can easily be adapted to take advantage of the rapidly increasing computing power by parallel processing. For example, modules performing CPU intensive tasks, such as data processing, system identification and 3D visualization can be handled by separate dedicated processors or computers in a distributed network computing environment. The processing and visualization modules are designed to have the capability to operate in real-time mode enabling the animation of bridge responses, such as the displacements, in near real-time as limited by the network speed.

The application platform consisting of the various data processing modules and analysis engines has been used in the research of the operational vibration behaviour of the Confederation Bridge and the characteristics of the field monitoring data presented in the preceding chapters.

\subsection{Confederation Bridge monitoring data}

The vibration monitoring instrumentation of the Confederation Bridge comprises 76 accelerometers distributed mainly along a typical structural unit of one portal frame unit and a simply supported drop-in expansion span. Vibrations of the girders are measured in the vertical and lateral directions, as shown in Figure 2-1(b), by piezoelectric and servo accelerometers. The voltage signals from the accelerometer sensors are conditioned and filtered on site for anti-aliasing by an 8-pole $50 \mathrm{~Hz}$ low-pass Bessel filter prior to analog-todigital (A/D) conversion by the data loggers. Signals are sampled and digitized by a network of distributed high speed data loggers located inside the structure. The data loggers may be programmed to either collect data on a continuous or triggered basis. The triggering of the 
data acquisition can either be manual or automatic upon detection of specific dynamic events. Data are collected at user specified sampling rates that typically vary between $100 \mathrm{~Hz}$ and 167 $\mathrm{Hz}$. In triggered acquisition mode, the time interval of data recording usually varies between 90 seconds and 15 minutes and includes a 30 second pre-trigger buffer. Sampled data are stored temporarily in logger memory until retrieval at specified scheduled intervals from a remote network computer assigned to the control and operation of each data logger. The assigned computer retrieves the data from the data logger and sends them to a centralized platform where they are made accessible to researchers over the internet. Figure 4-1 illustrates the flow of the dynamic monitoring data. Additional details of the monitoring system setup have been described by Cheung et al. (1997) and Montreuil et al. (1998).

The monitoring data contained in data file packets transmitted to Carleton University via internet from the bridge site are output-only vibration responses of the Confederation Bridge to ambient excitation conditions. Typical ambient operational loading conditions include traffic, wind, current and ice floes. The size of a typical 10 minute recording event, composed of three datasets from three separate data loggers, is approximately $48 \mathrm{MB}$. This corresponds to a total data volume of $7 \mathrm{~GB}$ per day if the monitoring system were to be operated under continuous data collection mode. The current state of operation uses triggered acquisitions of vibration data at a high sampling rate along with continuously recorded statistical information such as mean, maximum, minimum and standard deviation, mainly for system diagnostic purposes.

In a vibration monitoring system, acceleration data retrieved from the central on-site data collection platform, after analog-to-digital (A/D) conversion by the data loggers, often require some processing to prepare the data for analysis and interpretation. In the vibration 
monitoring system of the Confederation Bridge the collected raw data may have some of following characteristics that need to be resolved at the processing stage:

- There may be a slight lack of time synchronization in the system clocks of various dataloggers in the distributed monitoring system. Although the triggering of data collection occurs simultaneously at different data-loggers of the monitoring system, the time stamps contained in the separate data files from different loggers may differ slightly because of small time differences in the data-logger clocks.

- There may be small gaps in the data files due to samples missed during high speed data acquisition. This is because the data logger task sequencer executes the data collection program at a rigid precisely controlled schedule that runs according to the CPU clock speed. Consequently, if the data-logger processor is overburdened with additional operation commands beyond the normal data collection procedures one or a few samples may be skipped in the data records. As an example, this tends to occur when the logger is switching between different data collection modes at the end of the 30 second pre-trigger data buffer.

- There may be duplication of data samples in small segments of the data files which can be caused by the duplicate retrieval of data records from logger memory by the logger control software that retrieves data from the logger to the on-site logger control computer.

- Accelerometer time history signals may have a constant baseline offset (DC component) resulting from slow drift of the voltage measurement baseline over time.

- There may be some high frequency noise contamination in the data, such as those caused by electrical noise at harmonics of the electrical frequency. 
- Different data loggers may be set to acquire vibration response data at different sampling rates in order to optimize the efficiency and processing loads of the data acquisition programs in different data loggers.

- Data from a dynamic event recorded by a data logger may be partitioned into multiple separate files which may be caused by communication interruption or conflict developed during scheduled automatic data collection and data transmission.

- Data files may contain data from multiple triggered dynamic events.

A data checking module has been developed with the functionality to verify the validity of the data and to identify the potential problems in the data and then automatically correct the detected problems. The following sections of the chapter present the key operations and algorithms of the data management, processing, analysis and visualization modules.

\subsection{Data processing}

File sorting and processing of the raw monitoring data is performed in order to correct the above listed problems and to produce suitable outputs for data analysis and visualization. The file sorting operation organizes the data files into appropriate input files for the processing engine. File sorting and processing tasks include:

- The identification and separation of different data events and data event segments in the data files. A data event is taken as the continuous data of a specified duration, usually ten minutes, collected upon detection of a significant dynamic event or upon manual triggering of the data acquisition system.

- The assembling of matching data event segments to form complete data events of proper duration. 
- Synchronization of data events from different loggers into full datasets corresponding to the same dynamic event. A dataset is the synchronous responses of all bridge sensors for a specified duration.

- Patching small data gaps. Where data samples are found to be missing, a cubic polynomial interpolation algorithm using a piece-wise cubic hermit interpolating polynomial patches small data gaps by taking a set number of samples before and after the missing samples in the interpolation.

- Purging of duplicate data records. Data records are examined in chronological order to find potential duplication in the data records. A duplicate record exists when the sampling interval, calculated from the time-stamps of successive data samples, is found to be negative. The algorithm will then compare the potential duplicate records with earlier records to find a match. When a match is found the duplicate record is purged from the dataset.

- Baseline adjustment of the accelerometer time-history signals, by detrending. When the acceleration signals are to be integrated to obtain displacements, the baseline is corrected by means of high-pass filtering (high-pass 6th order Chebyshev Type II filter with a 0.1 $\mathrm{Hz}$ stop-band edge frequency).

- Conversion of the data to engineering units. For this purpose the program refers to a channel specification table containing the appropriate calibration factors and other channel specific information.

- Resampling of data to a common sampling rate. A dataset for a dynamic event consists of data collected simultaneously upon triggering by multiple data loggers which may operate at different sampling rates. To synchronize the signals from different data loggers to form a compatible consistent dataset, the minimum sampling rate among the 
loggers is determined and signals from loggers with higher sampling rates are subjected to a downsampling operation. This downsampling operation lowers the sampling rate of the signals of a given data logger by the ratio of integers $a / b$, where $\frac{a}{b}=\frac{\Delta t_{i}}{\Delta t_{\max }}, \Delta t_{i}$ is the sampling interval of logger $i$ and $\Delta t_{\max }$ is the maximum sampling interval among the different data loggers. An interpolation algorithm first up-samples the signal by the integer factor $a$, and then down-samples it by the integer factor $b$. As in any type of downsampling, filtering prior to downsampling is needed to prevent aliasing. In this case, a finite impulse response (FIR) filter is used to low-pass filter the up-sampled signals. The filter design characteristics are determined based on the downsampling ratio $(a / b)$. The phase delay introduced by the filter is compensated.

- Decimation of data. The typical sampling rate for the Confederation Bridge monitoring project is $125 \mathrm{~Hz}$, while the frequency content of interest of the structure is primarily below $15 \mathrm{~Hz}$. Consequently, processing and output computations of the data can be economized substantially by reducing the amount of data samples without compromising accuracy in the computations. Prior to downsampling, signals are low-pass filtered for anti-aliasing. The filter specifications and cut-off frequency can be customized depending on the desired output characteristics. By default, an eight order Chebyshev type I filter is used. The default cut-off frequency $\left(F_{i}\right)$ for the filter is $0.8\left(\frac{F_{s}}{2 r}\right)$, where $F_{s}$ is the original sampling frequency and $r$ is the decimation factor. The factor of 0.8 is applied to obtain a conservative cut-off frequency. Normally a decimation factor of three is used so, if $F_{s}=$ $125 \mathrm{~Hz}$, then $F_{c}=16.7 \mathrm{~Hz}$. The Chebyshev filter has a non-linear phase response. To prevent this from affecting the data, the filtering of the signals is carried out in both 
forward and reverse directions, which cancels out the phase distortion. After filtering, the filtered data are down-sampled by taking every $r^{\text {th }}$ sample of the filtered time histories.

- Integration of data signals. To obtain the displacement response of the structure from the acceleration time histories, signals are doubly integrated using a simple cumulative trapezoidal numerical integration (CTNI) method. Precautions with low frequency content need to be taken when integrating a signal because any DC component or nearzero frequency content is amplified during an integration operation. To remove the lowfrequency components, a sixth order Chebyshev type II high-pass filter is used prior to the first and after each integration operation. The algorithm operations for double integration are listed as follows

- High-pass filtering of the acceleration data.

- Integrating the filtered acceleration data with the CTNI method yielding the velocity responses.

- High-pass filtering the velocity signals to remove unwanted low frequency components resulting from the first integration.

- Integrating the filtered velocity using the CTNI method yielding the dynamic displacement responses of the structure.

- High-pass filtering the displacement signals to remove unwanted low frequency components resulting from the second integration.

The dynamic displacement responses obtained above can be animated on screen, which provides insights into the bridge behaviour under the different loading scenarios. The number of frames per second that can be animated depends on the available computing resources. To enable response at real-time mode, the displacement responses often have to 
be downsampled further. For structures with low-frequency response characteristics such as the Confederation Bridge, this does not lead to loss of important visual information.

All the above data file sorting and processing algorithms are integrated into two GUI modules, as shown in Figure 4-2. When the processing operations are being performed in the background, feedback and update on the status of the tasks performed is provided to the user through the GUI on screen. The data processing module generates outputs of acceleration and displacement time histories ready for further data analysis or visualization.

\subsection{Data visualization}

The visualization modules include a data plotting tool that provides a convenient environment for the exploration of large datasets within a reasonable time frame. Using the developed GUI, displacement and acceleration time histories and spectral plots can be easily manipulated to obtain the desired information on the behaviour of the structural system. Data may be viewed at any intermediate stage of processing to qualitatively evaluate the processing results. Different channels may be plotted simultaneously in the same figure providing easy comparison.

A second visualization module includes a 3D bridge model for animation of bridge displacement responses and mode shapes. Bridge responses during a dynamic event such as a windstorm, an earthquake or simply during normal operational conditions can be animated for more effective visualization and interpretation of the results, which provides valuable insight into the bridge behaviour. The animation module permits flexible user interaction. Parameters of the animations include scaling factor, view angle and playback speed. There is also an option to record animation sequences for playback on common media players.

The modules for data visualization help to facilitate the extraction of engineering information from the large datasets of continuous monitoring data for both research and 
operation of the bridge facilities in a timely manner, thus greatly adding engineering value to the monitoring data. Figure 4-3 (a) \& (b) show screen captures of the plotting and animation visualization modules.

\subsubsection{Real-time visualization}

The data processing and response animation modules are designed and adapted to have the operating capability to process and display the bridge vibration responses in a real-time mode. With the data collection process operating continuously and the data transmission procedure from the data loggers to the data control computer automated, the structural health monitoring application platform discussed herein is designed to receive a continuous incoming stream of data files. Recursive periodic search of designated data receiving directories is carried out to check for newly arriving data files in the host computer of the application platform. These are sorted and redirected to the data processing module and subsequently to the data visualization module for graphical display or plot of the bridge vibration motions in near real-time as limited by the network delay. The corresponding data flow is shown schematically in Figure 4-4. With this capability, responses of the bridge under normal or severe conditions may be viewed and assessed by operators of the bridge with minimal time delay. The ability to visualize the bridge response in near real-time adds a significant amount of engineering value to the monitoring data especially during the occurrence of major events such as hurricanes, earthquakes or ship collisions.

In general, the data visualization modules facilitate the timely extraction of engineering information from the large datasets collected by a continuous monitoring system. Timely extraction of engineering information from the bridge response monitoring data is potentially very useful for the operation and management of bridge facilities. For example, a warning system can be implemented using the extracted information to alert facility 
operators if it is detected that a certain response value exceeds a specified safety or threshold limit either during normal operation or upon the occurrence of an extreme event, such as an earthquake. In the future, given that important challenges currently existing in vibration based health monitoring can be overcome, it should be possible to integrate the real-time data processing modules with advanced damage detection algorithms to provide timely condition assessment of the monitored facilities based on evaluation of the continuous dynamic monitoring data

\subsection{Data analysis}

\subsubsection{Spectral analysis}

As a preliminary analysis of the bridge monitoring response signals, power spectral density (PSD) analysis of the monitoring data can be conducted to identify the dominant structural vibration frequencies and distribution of the energy of the signals in the frequency domain. In the developed computer application platform, PSD functions of the monitoring responses can be visualized through the data display module, as shown in Figure 4-3(a). The PSD function module can be applied to the analysis of the data signals at any intermediate or processing stage. The evaluation of the PSD estimates in the analysis of the Confederation Bridge monitoring data is based on the Welch method of dividing the sampled time series data into windowed overlapping segments using a Hamming window to minimize leakage in the FFT computation. The power spectra of the different data segments are averaged, yielding the power spectrum of the signal which is then divided by the sampling frequency resulting in the power spectral density estimate. The length of the data segments, which affects the frequency resolution of the spectra $(\Delta f)$, and the amount of overlap between 
adjacent data segments are user specified to optimize the trade-off between the number of segments for averaging and the frequency resolution of the spectra.

\subsubsection{Stochastic subspace identification application tool}

Determination of the structural vibration frequencies, mode shapes and damping ratios of the structural system from the monitoring data are important aspects of structural health monitoring as they are the fundamental parameters of vibration based structural condition assessment techniques. To obtain accurate values for structural frequencies, mode shapes and damping ratios of the structural system, an advanced system identification algorithm of stochastic subspace identification (SSI) has been implemented with a GUI that is seamlessly integrated into the main platform. The extracted properties can be used for condition evaluation of the structure.

For large-scale complex structures like the Confederation Bridge, it is either impractical or costly to carry out forced vibration measurements to extract the structural vibration parameters. Consequently, operational loadings, without detailed knowledge of the sources, must be relied upon as the input excitations. In the system identification analysis of operational vibration data, output-only system identification techniques are required. Among the system identification techniques proposed for civil engineering structural monitoring applications, the SSI methods are one of the most effective output-only identification techniques as compared to other available methodologies (Peeters and Ventura, 2003). Following the derivations by presented in Section 2.5.2, a data correlation variant of the SSI method has been implemented in the monitoring application platform reported herein, with the GUI shown in Figure 4-5.

The system identification GUI encompasses the process of identifying modes by pole-picking from the stabilization diagram, visualization of the identified mode shapes, and 
model validation by comparison of the non-parametric cross-spectra with plots of parametric cross-spectra estimates constructed from the identified modes.

\subsection{Research applications}

Advances in research on the behaviour of vibration monitoring data from real structures in the field are of great importance for the practical development of continuous structural health monitoring systems for structures. Detailed studies of the vibration behaviour of the Confederation Bridge have been significantly facilitated by the development of the computer tools described in this chapter. The complete integration of the various processing and analysis engines and tools into one central application greatly simplifies the objective of data mining. With file sorting and data processing fully automated, large numbers of datasets can be examined for enhancement of the knowledge database. This, together with the convenient GUIs for data analysis and visualization, which provide the ability to easily visualize different aspects of the data, including time histories, power spectra and animations of bridge responses or mode-shapes, means that meaningful results can be obtained with less effort in a shorter time frame.

With these new tools, detailed studies have been performed which have been presented in Chapter 2 and Chapter 3 of this thesis. Those studies illustrate the research opportunities that can be explored using the computer application tools presented in this chapter.

\subsection{Conclusions}

Continuous monitoring projects produce large amounts of data that require processing and analysis to be of any engineering value. The tasks of processing and analyzing the data can be time consuming and demanding for the analyst, hence a centralized 
and easy to use platform integrating all aspects of data manipulations from data management and processing to analysis and visualization is highly convenient. Such an application has been developed and the details have been presented in this chapter. The results of Chapters 2 and 3 illustrate the use and value of the application tools for research purposes.

The objective of the application is to facilitate not only the processing and evaluation of the monitoring data for research purposes, but also for timely practical applications and use of the information in bridge operations and maintenance. With this purpose in mind, the application has been adapted to allow the visualization of bridge responses in real-time. With this capability, during and immediately after the occurrence of extreme events such as windstorms, earthquakes or ship impacts, bridge response can be assessed with minimal time delay. The application platform can be expanded in the future to serve as a real-time condition assessment tool for the monitored structure, given that important challenges currently existing in vibration-based condition assessment can be overcome. 


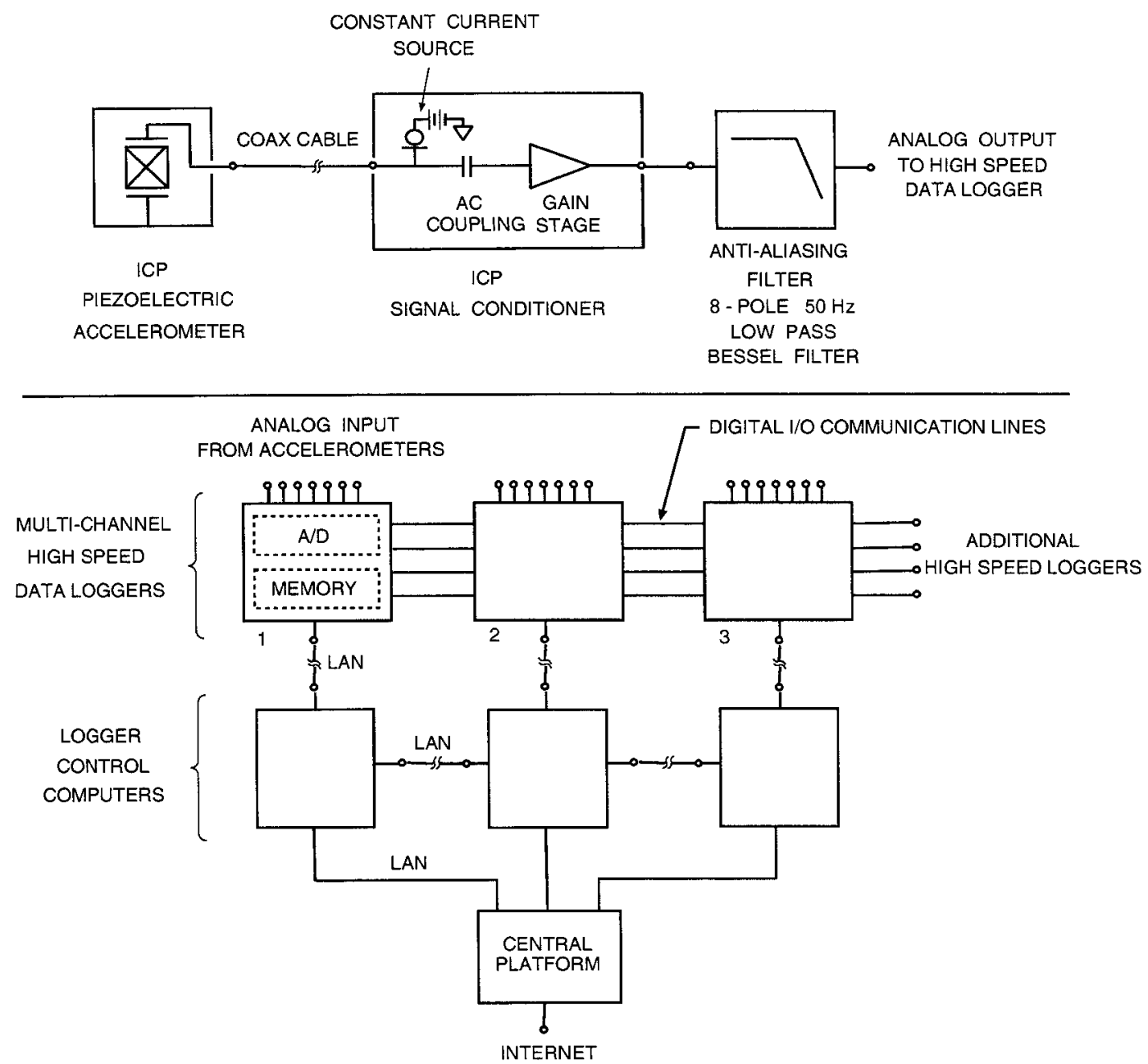

Figure 4-1: Schematic of Confederation Bridge dynamic data acquisition and transmission 

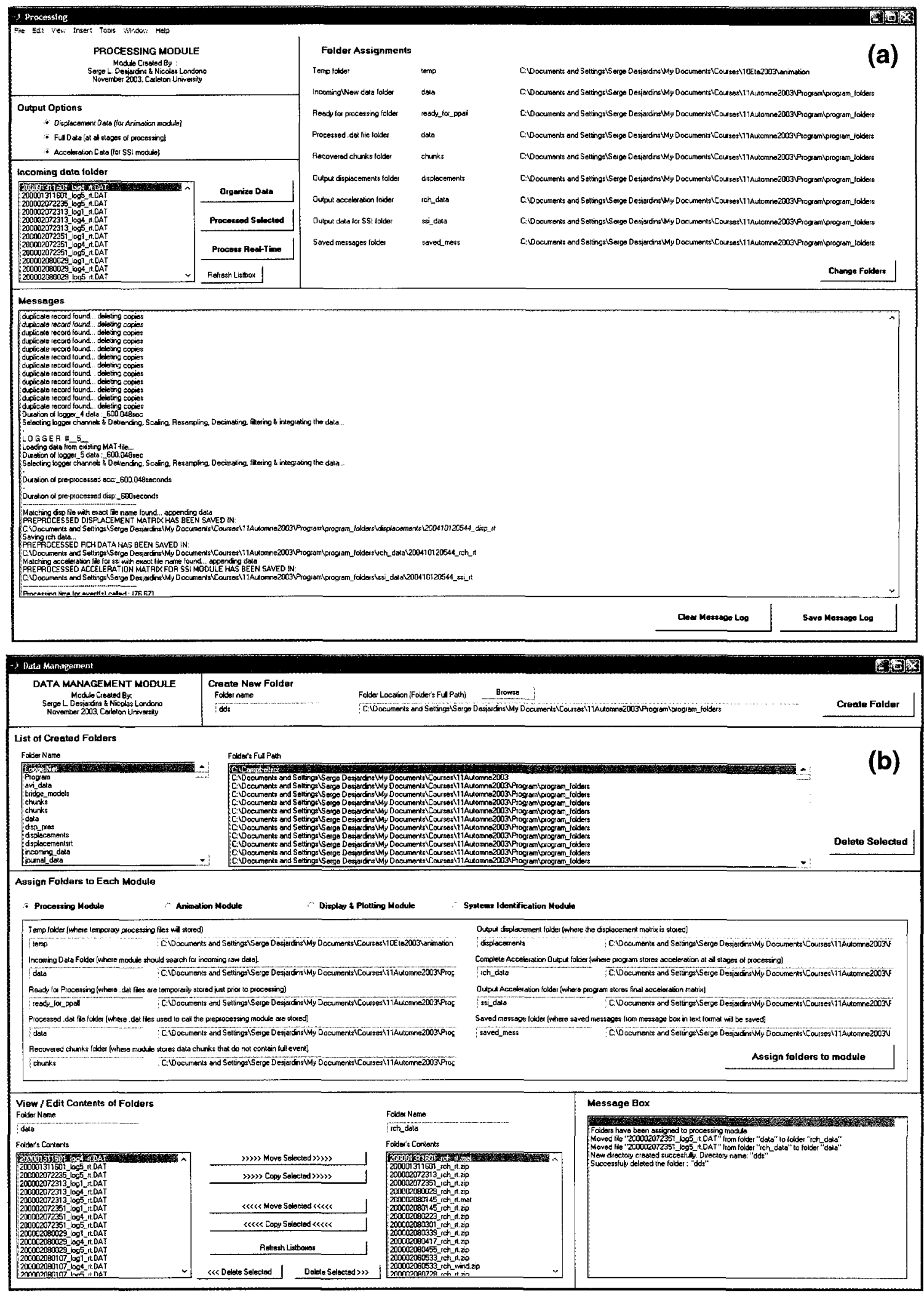

Figure 4-2: GUIs (a) data processing module; (b) file management module 

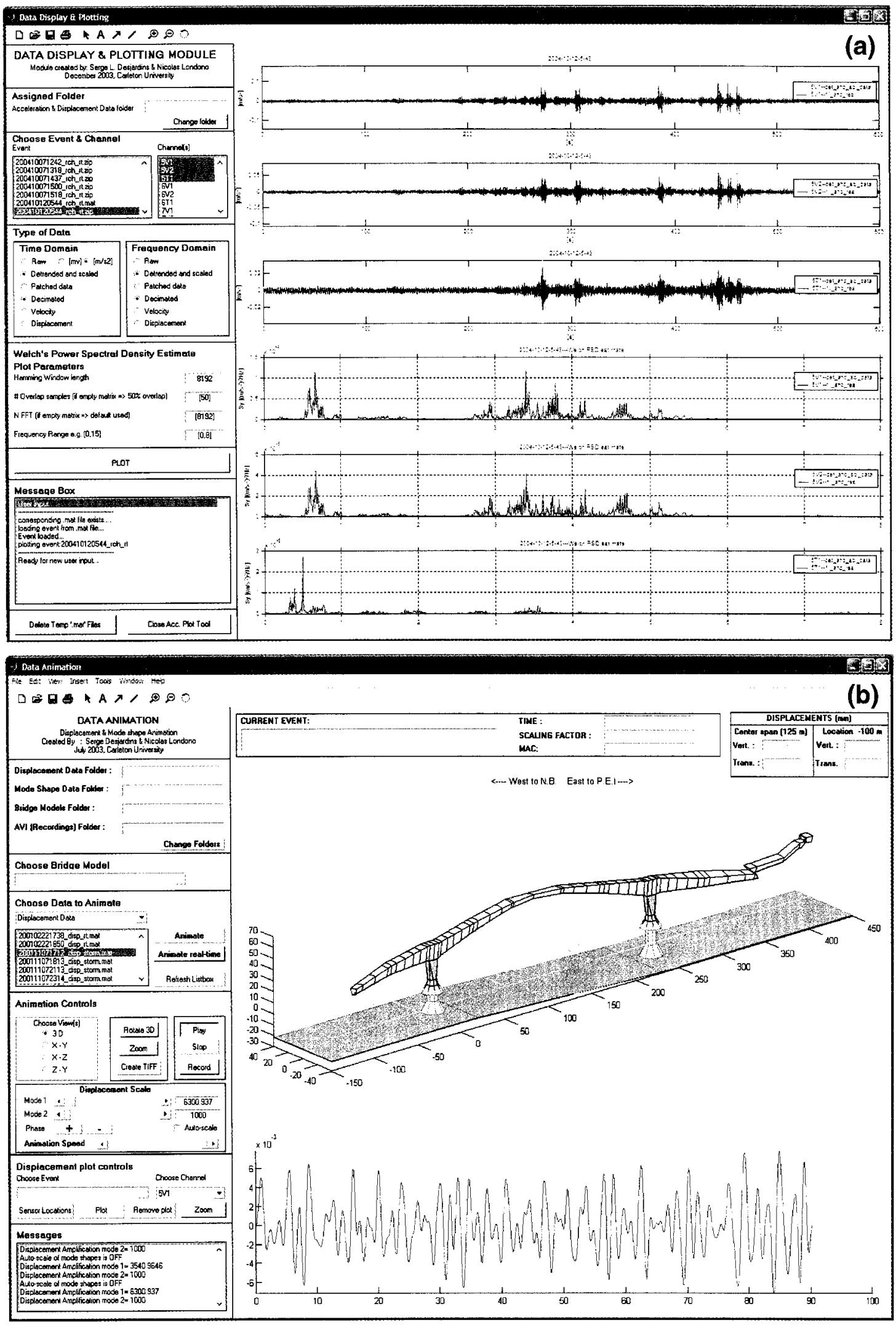

Figure 4-3: Graphical user interfaces (a) data display module; (b) animation module 


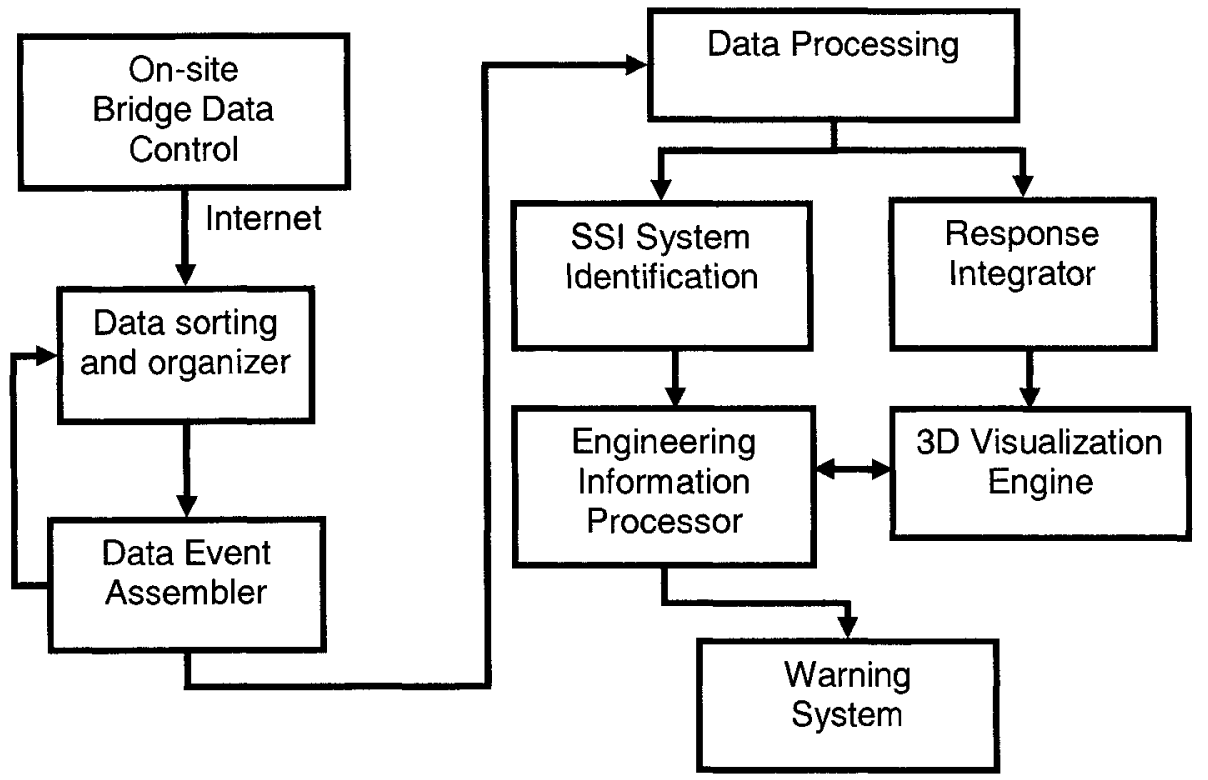

Figure 4-4: Schematic of real-time data processing and animation 


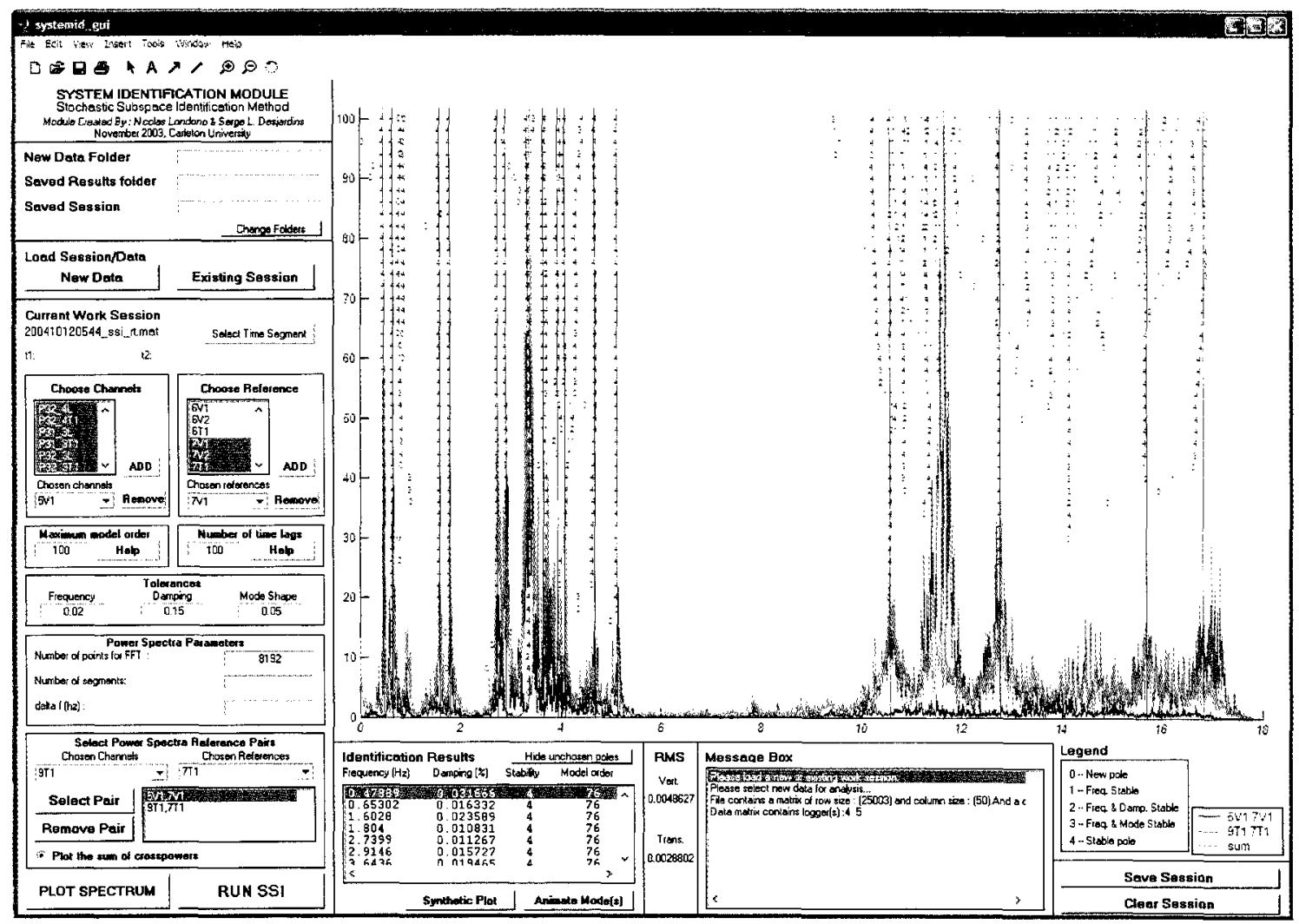

Figure 4-5: GUI for system identification data analysis with stabilization diagram 


\section{Chapter 5. Damage detection techniques}

\subsection{Introduction}

A large number and variety of structural damage detection methodologies has been proposed in recent years as reflected in recent literature review studies (Doebling et al. 1996, Sohn et al. 2003). The proposed methods range from non-model based damage index methods based directly on changes in the modal frequencies and mode shapes (and their derivatives), to flexibility matrix methods, and model-based updating techniques requiring the a-priori availability of a numerical model of the structure. Some other methods work by detecting damage as non-linearity in the structural responses. Various statistical and pattern recognition techniques for the discrimination of damage features have also been developed, including supervised and unsupervised techniques according to whether training data from the "damaged" structure is required or not. The numerical tools used by the different damage detection techniques include a wide array of techniques, including wavelet analysis, neural networks, genetic algorithms, Kalman filtering, empirical mode decomposition and control charts, just to name a few. Despite the apparent degree of advanced development and level of sophistication, it should be noted that most of the proposed damage detection techniques have only been tested in relatively simple simulations or in laboratory specimens. No single technique has yet brought about a significant consensus in the research community as being a robust and dependable damage detection technique, and the question of which is the "best" damage detection technique remains open for debate.

Given the large number and variety of techniques, it is not feasible to single-handedly and thoroughly assess the practical applicability of all the different damage detection techniques. Rather, one has to narrow down the scope to just a few promising techniques. 
Here, methods drawn from two of the main classical families of damage detection, namely model-based and non-model based, are considered. The methods are selected based on findings and observations on their performance as reported in the literature (Teughels et al. 2002, Wang et al. 2000, Humar et al. 2006).

In Chapter 6, the performance and reliability of a selected damage detection technique is evaluated through computer simulation studies in the presence of realistic variability in the data, using the findings obtained from the monitoring data of the Confederation Bridge and the variability model of Chapter 3. In the simulations, different damage scenarios are introduced in a finite element model of the Confederation Bridge, thus taking advantage of the existing FE models of the structure, which have previously been found to be reasonably accurate after validation against the monitoring data, as discussed in Chapters 2 and 3. The selected damage detection technique is used in the analysis of the "data" obtained from the finite element model simulations to evaluate their performance and sensitivity to uncertainties. Since the simulations incorporate a variability model based on actual monitoring data, the results can be expected to reflect what would be obtained in practice.

The following specific measures are taken to ensure that the damage detection simulations are realistic as possible:

- The modal vibration properties before and after damage are artificially contaminated with noise based on the variability model proposed in Chapter 3, which reflects the uncertainties in the field observed behaviour of the long-term monitoring data.

- Only a small subset of the vibration modes are used in the damage detection algorithms. The modes to be used are those which can be consistently extracted from the monitoring data, in accordance with the results presented in Chapter 2. 
- The mode shapes are reduced to a just a small subset of translational DOFs to simulate the limited availability of measurement locations.

In the following sections three different damage detection algorithms are discussed from which the most promising damage detection algorithm is selected for numerical evaluation. The three candidate methods as well as their advantages and disadvantages are discussed in detail. The candidate methods are: (1) a recently introduced variant of the model updating method using so-called "damage functions" as proposed by Teughels et al. (2002); (2) the so called "damage index" method, based on modal strain energies, which was originally proposed by Stubbs et al. (1995); and (3) a flexibility-based method, as proposed by Wang et al. (2000), plus a variant of the method using Damage Locating Vectors (Bernal, 2006). All of these methods have demonstrated promising performance in recent evaluations (De Roeck 2005, Humar et al. 2005, Wang et al. 2000, Gao and Spencer 2006). The comparison study presented here aims to determine which method has the most practical potential for vibration based condition assessment of bridges by identifying the strengths and weaknesses of each approach as well as any potential difficulties for practical implementation.

\subsection{Damage detection via finite element updating}

\subsubsection{Introduction}

Finite element updating is a well-established methodology for the solution of the inverse problem of calibration of finite element models based on measured data. Numerous variations of this method have been proposed (e.g. Maia et al. 1997, Friswell and Mottershead 1995). This branch of structural dynamics was initially developed with the intention of obtaining improved structural models to be used for improved response estimation and design. While the FE updating methodology is not originally intended for damage detection 
applications, the concept of the method is directly applicable to the damage detection problem in structural health monitoring. In the damage detection problem or structural condition assessment application, the structural model parameters are updated to match the model behaviour to that of the "damaged" structure. The updates represent the deteriorated or damaged condition of the structure.

FE updating falls within the category of model-based damage detection techniques, which require a numerical model of the structure. The advantage of model-based techniques is they are relatively simple conceptually and thus facilitate the interpretation of the results. In addition, FE updating procedures require neither mode shape expansion or reduction, nor mass-normalization of the measured mode shapes, thus eliminating significant numerical error in these operations. Despite these advantages, the efficiency of the FE updating method is dependent on selecting an appropriate model to represent the actual behaviour of the monitored structure. Another consideration is that model-updating algorithms often require the solution of a non-linear optimization problem which may not be straightforward.

Among the optimization-related challenges the following are the most important:

- The definition of an appropriate and well-behaved objective function. This means that the function, which can be thought of as a surface in the n-dimensional space of the optimization variables, should neither be too flat nor too crooked, and it should have a unique well-defined minimum in the domain of interest (the ideal case would be a function resembling a smooth parabola). The definition of the objective function includes the selection of:

- Structural properties for the formulation of the objective function. The comparison between the numerical model and the measured data from the real structure is usually expressed in terms of some kind of structural 
properties such as eigenvalues, eigenvectors, modal strain energies, flexibility coefficients, FRF's, etc.;

- The type of norm used for the objective function. The norm measures the distance between the measured structural properties and those of the FE model;

- The selection of appropriate optimization variables or updating parameters. The parameters to update in the model may include support or joint stiffness, bending, torsional, or axial element stiffness, damping characteristics, beam offsets, etc. The number of optimization variables should be kept to a minimum to avoid ill-conditioning while at the same time ensuring that changes in the selected variables can properly account for the necessary adjustments in the model to match the measured behaviour. A difficulty in applying the finite element updating technique is the relatively low information content of the measurement data as compared to the relatively large number of variables that are potentially updateable in the finite element model. This situation often leads to ill conditioning in the associated numerical optimization problem, which can make it difficult or impossible for the process to converge to the actual solution. To overcome this difficulty, either the quantity of measured data can be increased or the number of optimization variables can be reduced. In practice, increasing the quantity of measured data tends to be expensive, so the latter approach is generally preferred. Different approaches for the selection of appropriate updating parameters are discussed by Friswell et al. (2001). 
- The convergence to local minima. This can be avoided through the use of "global" optimization methods (such as Genetic Algorithms, Simulated Annealing, Coupled Local Minimizers, etc.) but these tend to be more expensive computationally.

- The difficulty of obtaining a well-calibrated model. Normally, the model to be used for damage detection must be first calibrated using data from the undamaged structure. A well-calibrated model should be able to match the measured behaviour (and ideally also any un-measured behaviour) as closely as possible. If using eigenvalues and mode shapes for calibration, ideally the eigenfrequency and mode shape residuals remaining after calibration should be small and uniform across the different vibration modes. In practice this may not be easy to achieve and it will depend to a large extent on the adequacy of the original numerical model. It should be mentioned however, that in damage detection, as long as these calibration errors or updating residuals are persistent and biased errors they will tend to cancel out in the second updating process.

The FE updating method involves the solution of two different inverse problems. First, the problem is to identify an experimental modal model of the structure from the vibration measurements, and secondly, the problem is to improve and update the parameters of the finite element model based on the identified modal properties. When using finite element updating for structural health monitoring, the finite element model is first tuned using measurement data from the "undamaged" structure. Then, in a second updating process, the updated model, called "reference" model, is tuned using measurement data from the "damaged" structure. The changes (or updates) in the finite element model parameters obtained from the second updating process, usually in the form of element stiffness reduction factors, give an indication on the localization and severity of the damage. Since 
both location and severity of the damage are identified, the finite element updating approach corresponds to a level III damage detection methodology.

A few FE updating approaches have shown good damage detection potential for applications involving output-only field data of civil engineering structures (Friswell et al. 1996, Brownjohn et al. 2001, Teughels et al. 2002, Jaishi and Ren 2006, Wu and Li 2006). An adaptation of the method using a parameter reduction technique called "damage functions", proposed by Teughels et al. (2002), has been successfully applied to identify artificially induced damage in the benchmark Z-24 highway bridge in Switzerland (Mack and De Roeck 2003). The "damage functions" decrease the number of optimization variables by approximating the actual damage pattern of the structure through parameterized shape functions that are superimposed on the finite element mesh. The damage shape functions represent, for example, the variation of stiffness correction factors throughout the domain of the structure, using a relatively small number of parameters. With this simple parameter reduction technique, the conditioning of the optimization problem is significantly improved. The FE updating approach using damage functions is summarized in the following sections.

\subsubsection{Objective function}

The first step in finite element updating is the definition of the objective function which is to be used as the basis for the subsequent optimization process. The objective function typically represents the discrepancies between the numerical and experimental modal data and is usually expressed as a sum of squared residuals as follows,

$$
f(\boldsymbol{\theta})=\frac{1}{2} \sum_{i=1}^{m}\left[z_{i}(\boldsymbol{\theta})-\widetilde{z}_{i}\right]^{2}=\frac{1}{2} \sum_{i=1}^{m} r_{i}(\boldsymbol{\theta})^{2}=\frac{1}{2}\|\mathbf{r}(\boldsymbol{\theta})\|^{2}
$$


where $z_{i}(\theta)$ represents a quantity obtained from the finite element model that is a nonlinear function of the optimization variables $\theta$, and $\widetilde{z}_{i}$ represents the corresponding measured quantities. The residuals $r_{i}$ are defined as: $r_{i}=z_{i}-\widetilde{z}_{i}$. In order to obtain a unique solution, the number of residuals $(m)$ must be greater than the number of optimization variables $(n)$, i.e. the problem must be overdetermined. In practice, it is often advantageous to give more weight to some residuals than others according to their importance and amount of noise. The weighted least squares problem is formulated as follows:

$$
f(\boldsymbol{\theta})=\frac{1}{2} \sum_{i=1}^{m}\left[w_{i}\left(z_{i}(\boldsymbol{\theta})-\widetilde{z}_{i}\right)\right]^{2}=\frac{1}{2} \sum_{i=1}^{m}\left[w_{i} r_{i}(\boldsymbol{\theta})\right]^{2}=\frac{1}{2}\left\|\mathbf{W}^{\frac{1}{2}} \mathbf{r}(\boldsymbol{\theta})\right\|^{2}
$$

where the weighting matrix $\mathbf{W}$ is a diagonal matrix containing the squared weights.

$$
\mathbf{W}=\left[\begin{array}{lll}
\ddots & & \\
& w_{i}^{2} & \\
& & \ddots
\end{array}\right]
$$

\section{Residuals}

Different quantities can be used in the formulation of the objective function residuals, including eigenvalues, mode shapes, modal scale factors, normalized mode differences, modal strains, modal strain energy, and modal strain energy change ratios. A relatively simple formulation of the residuals using just the eigenvalues and the un-scaled mode shapes gives good results while facilitating the derivation of the gradient functions which are needed in the numerical optimization process. This residual vector is expressed as follows:

$$
\mathbf{r}(\boldsymbol{\theta})=\left[\begin{array}{l}
\mathbf{r}^{f}(\boldsymbol{\theta}) \\
\mathbf{r}^{s}(\boldsymbol{\theta})
\end{array}\right]
$$

where $\mathbf{r}^{f}(\boldsymbol{\theta})$ is a vector of eigenfrequency residuals and $\mathbf{r}^{s}(\boldsymbol{\theta})$ is a vector of mode shape residuals, which are defined respectively as follows: 


$$
\begin{aligned}
& r_{i}^{f}(\boldsymbol{\theta})=\frac{\lambda_{i}(\boldsymbol{\theta})-\tilde{\lambda}_{i}}{\widetilde{\lambda}_{i}}, \quad i \in\left\{1, \ldots, m_{j}\right\} \\
& r_{i}^{s}(\boldsymbol{\theta})=\frac{\psi_{i}^{l}(\boldsymbol{\theta})}{\psi_{i}^{r}(\boldsymbol{\theta})}-\frac{\widetilde{\psi}_{i}^{l}}{\widetilde{\psi}_{i}^{r}}, \quad i \in\left\{1, \ldots, m_{s}\right\} ; l \in\left\{1, \ldots, n d o f_{j}\right\}, l \neq r
\end{aligned}
$$

where $\lambda_{i}(\boldsymbol{\theta})=\left(2 \pi f_{i}\right)^{2}$ is the $i^{\text {th }}$ eigenvalue from the finite element model, $\tilde{\lambda}_{i}$ is the corresponding value identified from the experimental data, and $m_{f}$ is the number of eigenvalues used in the computation of the residuals. In the formulation of the mode shape residuals, $\psi_{i}^{l}(\boldsymbol{\theta})$ represents the $t^{\text {th }}$ mode shape coefficient of the $\mathrm{i}^{\text {th }}$ mode shape, while $\psi_{i}^{r}(\boldsymbol{\theta})$ represents the reference mode shape coefficient; $m_{s}$ is the number of mode shapes considered for the computation of residuals and $n d f_{j}$ is the number of DOFs of the $i^{\text {th }}$ mode. Since mass normalized modes cannot be obtained from output-only vibration data, the mode shape coefficients are normalized to one in the reference node, which is normally taken as the node with the largest amplitude (or a selected node with relatively large amplitude). In Equation (5-6), the same reference node is used in the mode shape residual calculation for each pair of numerical and experimental modes.

Since the sequence of mode-shapes may be different between the modes obtained from the finite element model and the identified modes from measurement data, a prior mode-pairing step between the analytical and experimental mode sets of mode shapes is necessary. This can be carried out by means of the MAC (Eq. (3-1)). For an experimental mode, the corresponding analytical mode is the one that shows the highest MAC value with that experimental mode. 


\subsubsection{Optimization variables}

In finite element updating one or more physical parameters of the structure (e.g. foundation or support stiffness, elastic modulus, shear modulus, mass, wall thickness, inertia, etc.) are corrected where they are found result in discrepancies in the measurement data. The number of parameters that can potentially be updated is typically very large. In principle, every parameter in each of the system matrices can be updated. However, to avoid illconditioning of the parameter estimation problem, in practice only a relatively small number of parameters are selected to be updated, corresponding to properties of the model which are actually suspected to deviate significantly from the real values. Hence, engineering judgement is required to determine which parameters should be selected for update and which not. One example of how the number of updating variables is reduced is through the commonly adopted assumption that the mass of the structure, as determined from the design drawings, is known with relatively high accuracy and also that it is not expected to change significantly from the initial value as a result of damage to the structure. Hence, in this case, only the stiffness properties of the finite element model need to be updated.

The updating amount for a given physical parameter $X$ is expressed in terms of correction a factor $c_{e}^{X}$,

$$
c_{e}^{X}=-\frac{X_{e}-X_{e}^{r e f}}{X_{e}^{r e f}}
$$

which represents the relative difference between the value of the parameter $X$ in element $e$, with respect to its reference value.

If parameter $X$ is linearly related to the element stiffness matrices, then the correction factors can be applied directly to the element stiffness matrix as follows

$$
\mathbf{K}_{e}=\mathbf{K}_{e}^{r e f}\left(1-c_{e}\right)
$$


where $\mathbf{K}_{e}^{r e f}$ and $\mathbf{K}_{e}$ are the reference and updated element stiffness matrices respectively, which in turn yields the following expression for the global stiffness matrix:

$$
\mathbf{K}=\mathbf{K}^{u}+\sum_{e=1}^{n_{e}} \mathbf{K}_{e}^{r e f}\left(1-c_{e}\right)
$$

where $\mathbf{K}$ is the global stiffness matrix and $\mathbf{K}^{\prime \prime}$ represents the stiffness matrix of those elements which are not involved in the updating process and hence their contribution remains unchanged.

Typically finite element models are built with a relatively large number of elements to give sufficient accuracy in modelling the behaviour of the structures. This implies that if the element correction factors $c_{e}$ were to be used directly in the optimization problem, there would be a large number of optimization variables, which would in turn lead to numerical problems. As a result, damage shape functions are introduced to reduce the number of variables and thus overcome this difficulty.

In the formulation, the damage function discretizes a distribution of the correction factor $c_{e}$ over the finite element mesh by interpolation using a set of nodal parameters $n$ that is considerably smaller than $n_{e^{*}}$ Among the several different kinds of shape functions that have been proposed, here a piece-wise linear damage shape function is adopted because it is simple and generally versatile, which helps to ensure a relatively smooth and physically meaningful solution. In addition, the piece-wise linear damage function tends to produce a well-behaved objective function with a unique minimum which greatly facilitates the optimization process.

The piecewise linear global damage function is established as a linear combination of triangular shape functions as shown in Figure 5-1. The mapping of the piecewise linear damage function to the element correction factors can be expressed as follows 


$$
c_{e}=\mathrm{N}\left(\mathbf{x}_{e}, \boldsymbol{\theta}\right)=\sum_{j=1}^{n_{p}} \theta_{j} N_{j}\left(\mathbf{x}_{e}\right)
$$

where $\mathrm{N}$ represents the global damage shape function, $\mathbf{x}_{\ell}$ represents the coordinates at the center of element $e, N_{j}$ is a triangular shape function defined over the $j^{\text {th }}$ local portion of the finite element model, and $\theta_{j}$ is the corresponding amplitude of the local shape function, as illustrated in Figure 5-1. The shape function amplitudes, which are grouped in vector $\boldsymbol{\theta}$, are the actual optimization variables.

The accuracy of the updating result is determined by the coarseness of the damage function. A finer mesh can be adopted if higher accuracy is required to better approximate the actual continuous damage distribution by piecewise linear shape functions. Alternatively, higher order shape functions can be used. However, both of these options will result in a larger number of unknown parameters $\theta$ in the optimization process, which can have a negative impact the condition of the optimization problem. In practice, the refinement of the damage element mesh is limited by the quantity and quality of the measurement information, the more and better the information is, the finer the mesh can be. In the implementation of the algorithm, a compromised balance should be found between the fineness of the mesh and the condition of the optimization problem.

\subsubsection{Optimization algorithm}

The optimization problem to be solved is to find the set of optimization variables $\theta$ that minimize the sum of squared residuals given in Eq. (5-1). This problem can be stated as follows

$$
\min _{\boldsymbol{\theta}} \frac{1}{2}\|\mathbf{r}(\boldsymbol{\theta})\|^{2}
$$


This is a non-linear least squares problem and the solution may be found by using one of the several available iterative techniques. In general, the iterative techniques start from an initial estimate of the problem variables $\theta$ and then advance step by step towards the optimum solution $\theta^{*}$, which minimizes Eq. (5-1). The difference between the various existing approaches lies in the way each solution step is computed. But most of these iterative techniques are variations of the basic Newton method, also known as the pure Newton method. This method generates consecutive iteration steps by minimizing a quadratic model $q_{k}(\mathbf{p})$ that is built around the current iteration $\boldsymbol{\theta}_{k}$ as an approximation to the exact objective function $f(\boldsymbol{\theta})$. The quadratic model is expressed as follows

$$
q_{k}(\mathbf{p})=f\left(\boldsymbol{\theta}_{k}\right)+\nabla f\left(\boldsymbol{\theta}_{k}\right)^{T} \mathbf{p}+\frac{1}{2} p^{T} \nabla^{2} f\left(\boldsymbol{\theta}_{k}\right) \mathbf{p}
$$

where $\nabla f\left(\boldsymbol{\theta}_{k}\right)$ and $\nabla^{2} f\left(\boldsymbol{\theta}_{k}\right)$ are the gradient vector and Hessian matrix respectively, and $\mathbf{p}$ is the dummy set of variables. When the Hessian matrix $\nabla^{2} f\left(\boldsymbol{\theta}_{k}\right)$ is positive definite, a unique minimizer of the quadratic model, $\mathbf{p}_{k}^{N}$, can be found from the solution of the following $n \times n$ system of equations

$$
\nabla^{2} f\left(\boldsymbol{\theta}_{k}\right) \mathbf{p}_{k}^{N}=-\nabla f\left(\boldsymbol{\theta}_{k}\right)
$$

which are referred to as the Newton equations, where $\mathbf{p}_{k}^{N}$ is the solution step for the $\mathrm{k}^{\text {th }}$ iteration, called "Newton step". The improved optimization variables are obtained simply as follows

$$
\boldsymbol{\theta}_{k+1}=\boldsymbol{\theta}_{k}+\mathbf{p}_{k}^{N}
$$

For the case of a least squares objective function (Eq. (5-1)), the gradient vector and Hessian matrix of the objective function can be expressed in terms of the derivatives of the residuals as follows 


$$
\begin{aligned}
& \nabla f(\mathbf{x})=\mathbf{J}(\mathbf{x})^{T} \mathbf{r}(\mathbf{x}) \\
& \nabla^{2} f(\mathbf{x})=\mathbf{J}(\mathbf{x})^{T} \mathbf{J}(\mathbf{x})+\sum_{i=1}^{m} r_{i}(\mathbf{x}) \mathbf{G}_{i}(\mathbf{x})
\end{aligned}
$$

where the Jacobian matrix $\mathbf{J}(\mathbf{x})$ and the Hessian matrices $\mathbf{G}_{i}(\mathbf{x})$ contain the first order and second order derivatives of the residuals respectively, as follows:

$$
\begin{aligned}
& \mathbf{J}(\mathbf{x})_{n \times n}, \quad \mathbf{J}(\mathbf{x})_{i j}=\frac{\partial r_{i}(\mathbf{x})}{\partial x_{j}}, \quad i=1,2 \ldots, m ; j=1,2, \ldots, n \\
& \mathbf{G}_{i}(\mathbf{x})=\nabla^{2} r_{i}(\mathbf{x})_{n \times n}, \quad \mathbf{G}_{i}(\mathbf{x})_{j k}=\frac{\partial^{2} r_{i}(\mathbf{x})}{\partial x_{i} \partial x_{k}}
\end{aligned}
$$

The problem with the pure Newton method is that its convergence is not always guaranteed and it requires the calculation of the second order derivatives of the residuals (i.e. the Hessian matrices), with respect to the optimization variables which is often difficult or computationally expensive for large-scale problems. As a result several different methods have been proposed which modify the basic Newton method to improve its convergence characteristics and to avoid the direct computation of the second order derivatives. Among these techniques the Trust-region Gauss Newton method (or Levenberg-Marquardt method) is adopted for use in finite element updating algorithm because of the advantages that it requires only first derivative information, it has a relatively fast convergence rate, it is suitable for ill-conditioned optimization problems and its formulation remains relatively simple as compared to other methods.

The Gauss-Newton methods take advantage of the fact that for least squares problems the Hessian $\nabla^{2} f\left(\boldsymbol{\theta}_{k}\right)$ is a combination of first and second order information, as expressed in Eq. (5-16), and it may often be assumed the first order term dominates the second order term. This assumption is valid when the norm of the residuals at the solution 
is relatively small compared to the largest eigenvalue of $\mathbf{J}(\mathbf{x})^{T} \mathbf{J}(\mathbf{x})$ at the solution, which is most often the case.

Neglecting the second order term in Eq. (5-16), and substituting Eqs. (5-15) and $(5-16)$ in $(5-12)$ the quadratic model to be minimized at each iteration step becomes

$$
\begin{aligned}
q_{k}(\mathbf{p}) & =\frac{1}{2}\left\|\mathbf{r}\left(\boldsymbol{\theta}_{k}\right)\right\|^{2}+\left(\mathbf{J}\left(\boldsymbol{\theta}_{k}\right)^{T} r\left(\boldsymbol{\theta}_{k}\right)\right)^{T} \mathbf{p}+\frac{1}{2} \mathbf{p}^{T}\left(\mathbf{J}\left(\boldsymbol{\theta}_{k}\right)^{T} \mathbf{J}\left(\boldsymbol{\theta}_{k}\right)\right) \mathbf{p} \\
& =\frac{1}{2}\left\|\mathbf{r}\left(\boldsymbol{\theta}_{k}\right)+\mathbf{J}\left(\boldsymbol{\theta}_{k}\right) \mathbf{p}\right\|^{2}
\end{aligned}
$$

The minimization of this function over $\mathbf{p}$ is a relatively simple linear least squares problem, and the solution can be found by solving by the corresponding set of Newton equations

$$
\left(\mathbf{J}\left(\boldsymbol{\theta}_{k}\right)^{T} \mathbf{J}\left(\boldsymbol{\theta}_{k}\right)\right) \mathbf{p}_{k}^{G N}=-\mathbf{J}\left(\boldsymbol{\theta}_{k}\right)^{T} \mathbf{r}\left(\boldsymbol{\theta}_{k}\right)
$$

for which a closed form solution for the Gauss-Newton search direction, $\mathbf{p}_{k}^{G N}$, may be computed via one of the standard linear algebra techniques such as Cholesky, QR or SVD factorizations.

The only weakness of the Gauss-Newton method is that it is prone to lack of convergence in the case of a nearly rank-deficient Jacobian, which can occur in finite element updating problems due to the similarity in the sensitivity of the residuals to changes occurring in neighbouring elements. One approach to overcome this weakness is by using a "trust region" constraint, which essentially limits the size of the iteration steps $\mathbf{p}_{k}$ taken to ensure that they remain within the "trust region", where the quadratic model $q$ is an adequate representation of the actual objective function $f$. The trust region is usually a sphere, defined by $\|\mathbf{p}\| \leq \Delta_{k}$, where $\Delta_{k}>0$ is the trust region radius and it is adaptively defined at each iteration step. 
In such an implementation, a candidate for each new iteration step is computed by minimizing the quadratic model within the trust region, which can be written as follows

$$
\min _{\mathbf{p}} q_{k}(\mathbf{p})=\frac{1}{2}\left\|\mathbf{r}\left(\boldsymbol{\theta}_{k}\right)+\mathbf{J}\left(\boldsymbol{\theta}_{k}\right) \mathbf{p}\right\|^{2}, \quad \text { subjected to }\|\mathbf{p}\| \leq \Delta_{k}
$$

For a candidate to be accepted, it must produce a sufficient reduction of the actual objective function. Otherwise the candidate is rejected, the trust region radius is reduced and a new candidate is computed. On the other hand, if the candidate is accepted, it means that the quadratic model is adequately representing the objective function and thus the trust region is increased for the next iteration step. The above trust region optimization algorithm is available in the Matlab software and will be used here as part of the finite element updating process. A more detailed description of the trust region algorithm may be found in the reference by Nocedal (1999).

\subsubsection{Jacobian matrix}

The solution of the optimization problem (Eq. (5-11)) by the Trust region GaussNewton method requires the computation of the Jacobian matrix $\mathrm{J}\left(\boldsymbol{\theta}_{k}\right)$ (also known as "sensitivity matrix") at each iteration step. This matrix contains the first order derivatives of each of the residuals $r_{i}$ with respect to each of the optimization variables $\theta_{j}$;

$$
\mathbf{J}=\left[\begin{array}{cccc}
\frac{\partial r_{1}}{\partial \theta_{1}} & \frac{\partial r_{1}}{\partial \theta_{2}} & \cdots & \frac{\partial r_{1}}{\partial \theta_{n}} \\
\frac{\partial r_{2}}{\partial \theta_{1}} & \ddots & & \\
\vdots & & \ddots & \\
\frac{\partial r_{m}}{\partial \theta_{1}} & & & \frac{\partial r_{m}}{\partial \theta_{n}}
\end{array}\right]_{m \times n}
$$

As explained in Section 5.2.2, two types of residuals are employed: eigenvalue residuals $\mathbf{r}^{\mathrm{f}}$ and mode shape residuals $\mathbf{r}^{s}$. The relation between the optimization variables, i.e. the parameters 
of the damage function, and the residuals is not immediately obvious or direct. Hence, the derivatives are first computed at the element level. The sensitivities of the eigenvalues and mode shapes with respect to the element correction factors $c_{e}$ have been derived analytically by Fox and Kapoor (1968) and can be simplified to the following expressions:

$$
\begin{aligned}
\frac{\partial \lambda_{i}}{\partial c_{e}} & =-\boldsymbol{\varphi}_{i}^{T} \frac{\mathbf{K}_{e}}{\left(1-c_{e}\right)} \boldsymbol{\varphi}_{i}=-\frac{\boldsymbol{\varphi}_{i}^{T} \mathbf{F}_{i}^{e}}{\left(1-c_{e}\right)} \\
\frac{\partial \boldsymbol{\varphi}_{i}}{\partial c_{e}} & =\sum_{q=1 ; q \neq i}^{d} \frac{\boldsymbol{\varphi}_{q}}{\lambda_{i}-\lambda_{q}}\left(-\boldsymbol{\varphi}_{q}^{T} \mathbf{K}_{e}^{r e f} \boldsymbol{\varphi}_{i}\right)=\sum_{q=1 ; q \neq i}^{d} \frac{\boldsymbol{\varphi}_{q}}{\lambda_{i}-\lambda_{q}}\left(-\frac{\boldsymbol{\varphi}_{q}^{T} \mathbf{F}_{i}^{e}}{1-c_{e}}\right)
\end{aligned}
$$

where $\mathbf{F}_{i}^{e}=\mathbf{K}_{e} \boldsymbol{\varphi}_{i}$ represents the nodal forces of element $e$ under the deformation of the $i^{\text {th }}$ mode shape $\varphi_{i}$. It should be noted that in these analytical expressions, which are computed using the finite element model, the mode shapes are mass-normalized. This differs from the residual expression of Eq. (5-6) where both the analytical and experimental mode shapes are normalized to one in a reference node.

The derivatives of the residuals with respect to the element correction factors $c_{e}$, are obtained from Eqs. (5-5) and (5-6) as

$$
\begin{aligned}
& \frac{\partial r_{i}^{f}}{\partial c_{e}}=\frac{1}{\tilde{\lambda}_{i}} \frac{\partial \lambda_{i}}{\partial c_{e}} \\
& \frac{\partial r^{s}}{\partial c_{e}}=\frac{1}{\phi_{i}^{r}} \frac{\partial \phi_{i}^{l}}{\partial c_{e}}-\frac{\phi_{i}^{l}}{\left(\phi_{i}^{r}\right)^{2}} \frac{\partial \phi_{i}^{r}}{\partial c_{e}}
\end{aligned}
$$

Using these derivative expressions a Jacobian matrix $J_{c}$ with respect to the element correction factors $c_{e}$ can be obtained. To obtain the Jacobian with respect to the optimization variables $\theta$ an additional mapping operation is required, as follows

$$
\frac{\partial r_{i}}{\partial \theta_{j}}=\sum_{e=1}^{n_{e}} \frac{\partial r_{i}}{\partial c_{e}} \frac{\partial c_{e}}{\partial \theta_{j}}=\sum_{e=1}^{n_{e}} \frac{\partial r_{i}}{\partial c_{e}} \frac{\partial \mathrm{N}\left(\mathrm{x}_{e}\right)}{\partial \theta_{j}}
$$


In the case of a piece-wise linear damage function as defined in Eq. (5-10), Eq. (5-27) leads to

$$
\frac{\partial r_{i}}{\partial \theta_{j}}=\sum_{e=1}^{n_{e}} \frac{\partial r_{i}}{\partial c_{e}} N_{j}\left(x_{e}\right)
$$

which can be written in a matrix form as follows

$$
[\mathbf{J}]_{m \times n}=\left[\mathbf{J}_{c}\right]_{m \times n_{e}}[N]_{n_{e} \times n}
$$

If the residual vector is being weighted, as expressed in Eq. (5-2), then the Jacobian matrix also needs to be adjusted accordingly as follows,

$$
\mathbf{J}_{W}=\mathbf{W}^{\frac{1}{2}} \mathbf{J}
$$

The computational implementation of the FE updating algorithm described above for use in the damage detection simulation study of Chapter 6, is summarized in Figure 5-2.

\subsection{Modal strain energy damage index}

This method, which is also known simply as the damage index method, was originally proposed by Stubbs et al. (1995) for structures modelled by beam elements. Humar et al. (2006) generalized and applied the method to structures of general type and compared its performance against other damage detection methodologies using finite element simulations in which the modal parameters were artificially contaminated with noise. The other methods considered were mode shape curvature change, modal flexibility change, change in uniform flexibility shape curvature, modal residual vector, and a matrix updating technique. It was concluded that among the different methods considered the damage index appears to be the most reliable in predicting the location of damage and also the most tolerant to measurement errors. However, the findings on the performance of the method were conditional upon the type of measurement data used in the analysis. When using translational DOF measurement 
data (which in turn results in the identification of translational DOF mode shapes from the data), and then computing the modal curvatures by numerical differentiation, the results in the presence of simulated measurement noise were not satisfactory. On the other hand, when using direct strain measurements, which circumvents having to compute the curvatures by numerical differentiation, the damage was detected accurately. Hence, if the monitoring data include dynamic strain measurements, then the damage index method seems to be an attractive alternative for practical application. If not, then the method is not likely to yield reliable results. It is worth noting that the recent introduction and ongoing developments of fibre optic strain sensors could potentially result in significant improvements in the monitoring of dynamic strains, which would make the damage index method a more attractive alternative.

The formulation of the damage index technique is summarized below based on the work by Humar et al. (2006). For a beam type structure, the modal strain energy (MSE) of the structure deforming in its $i^{\text {th }}$ mode can be expressed as follows

$$
M S E_{i}=\int_{0}^{L} E I(x)\left[\psi_{i}^{\prime \prime}(x)\right]^{2} d x
$$

where $L$ is the length of the beam, $E I(x)$ is the flexural rigidity and $\psi_{i}^{\prime \prime}(x)$ is the modal curvature of the $i^{\text {th }}$ mode, which may be obtained either from direct strain measurements (if available, then this is the preferable option) or from numerical differentiation of the measured mode shapes (usually only translation DOFs are measured so this is typically the only available option but it is generally less accurate).

The contribution of the $j^{\text {th }}$ element of the beam, located between $x=a$ and $x=b$ to the total strain energy is given by 


$$
M S E_{i j}=\int_{a}^{b} E I_{j}\left[\psi_{i}^{\prime \prime}(x)\right]^{2} d x
$$

A ratio of the modal strain energy of element $j$ in the $i^{\text {th }}$ mode with respect to the total strain energy in the $i^{\text {th }}$ mode can be defined as follows

$$
F_{i j}=\frac{M S E_{i j}}{M S E_{i}}
$$

Expressions similar to Eqs. (5-31), (5-32) and (5-33) can be written for the "damaged" structure, denoted with the superscript "d" as follows

$$
\begin{aligned}
& M S E_{i}^{d}=\int_{0}^{L} E I^{d}(x)\left[\psi_{i}^{d \prime}(x)\right]^{2} d x \\
& M S E_{i j}^{d}=\int_{a}^{b} E I_{j}^{d}\left[\psi_{i}^{d^{\prime \prime}}(x)\right]^{2} d x \\
& F_{i j}^{d}=\frac{M S E_{i j}^{d}}{M S E_{i}^{d}}
\end{aligned}
$$

The following two assumptions are made in this method. First, it is assumed in Eq. (5-34) that $E I^{d}(x) \approx E I(x)$. This may be considered a valid approximation as long as damage is confined to a just a few elements. Secondly, it is assumed that

$$
F_{i j}^{d}(x) \approx F_{i j}(x)
$$

By substituting Eqs. (5-31) to (5-36) into (5-37) an damage index expression is obtained which gives the ratio between the flexural rigidities of the $j^{\text {th }}$ element before and after damage, based on the modal strain energy of the $i^{\text {th }}$ mode, as follows

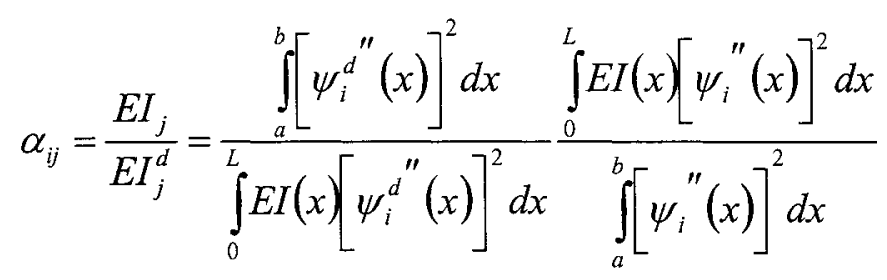


If the contributions of $n_{m}$ different measured modes are taken into account, then the damage index for the $\mathrm{j}^{\text {th }}$ element is expressed as follows

$$
\alpha_{j}=\frac{\sum_{i=1}^{n_{m}} \beta_{i j}^{d}}{\sum_{i=1}^{n_{m}} \beta_{i j}}
$$

with

$$
\beta_{i j}=\frac{\int_{a}^{b}\left[\psi_{i}^{\prime \prime}(x)\right]^{2} d x}{\int_{0}^{L} E I(x)\left[\psi_{i}^{\prime \prime}(x)\right]^{2} d x}, \beta_{i j}^{d}=\frac{\int_{a}^{b}\left[\psi_{i}^{d \prime}(x)\right]^{2} d x}{\int_{0}^{L} E I(x)\left[\psi_{i}^{d \prime}(x)\right]^{2} d x}
$$

The actual damage detection criterion is that elements for which $\alpha_{j}$ are relatively large as compared to the other elements are suspected to have damage.

Equation (5-39) is modified to avoid numerical problems in cases where the denominator is small, which may occur when the contribution of the $\mathrm{j}^{\text {th }}$ element to the modal strain energy of all the modes considered is small.

$$
\alpha_{j}^{*}=\frac{1+\sum_{i=1}^{n_{m}} \beta_{i j}^{d}}{1+\sum_{i=1}^{n_{m}} \beta_{i j}}
$$

Humar et al. (2006) extends the above formulation of the damage index method to structures of a general type by expressing the modal strain energy directly in terms of the stiffness matrix and mode shape displacements as follows

$$
\begin{aligned}
& M S E_{i j}=\boldsymbol{\varphi}_{i}^{T} \mathbf{k}_{j} \boldsymbol{\varphi}_{j} \\
& M S E_{i}=\boldsymbol{\varphi}_{i}^{T} \mathbf{K} \boldsymbol{\varphi}_{j}
\end{aligned}
$$


where $\mathbf{k}_{j}$ is the stiffness matrix of the $j^{\text {th }}$ element, and $\mathbf{K}$ is the stiffness matrix of the structure. Similar expressions can be written for the damaged structure except that in those expressions the stiffness matrix of the damaged structure, $\mathbf{K}^{\mathrm{d}}$, is considered to be approximately equal to $\mathbf{K}$, in analogy with the previous assumption of $E I^{d}(x) \approx E I(x)$. The damage index $\alpha$ is now defined in terms of the elemental stiffness matrices as

$$
\mathbf{k}_{j}^{d}=\frac{\mathbf{k}_{j}}{\alpha}
$$

Using a procedure similar to the one outlined above for beam type structures, an expression similar to Eq. (5-39) can be obtained. Hence, Eqs. (5-39) and (5-41) are still valid for structures of general type, except that $\beta_{i j}$ and $\beta_{i j}^{d}$ are now defined as follows

$$
\beta_{i j}=\frac{\boldsymbol{\varphi}_{i}^{T} \mathbf{k}_{j} \boldsymbol{\varphi}_{i}}{\boldsymbol{\varphi}_{i}^{T} \mathbf{K} \boldsymbol{\varphi}_{i}}, \quad \beta_{i j}^{d}=\frac{\boldsymbol{\varphi}_{i}^{d T} \mathbf{k}_{j} \boldsymbol{\varphi}_{i}^{d}}{\boldsymbol{\varphi}_{i}^{d T} \mathbf{K} \boldsymbol{\varphi}_{i}^{d}}
$$

When applying the damage index technique to a structure modelled by beam elements, there are two options available for the computation of the damage index. One approach is to calculate the modal curvatures by numerical differentiation and then use the beam type formulation of the method. The other alternative is to use an expansion/reduction technique (such as Guyan reduction, SEREP, etc.) to make the DOFs of the experimental modes compatible with the DOFs of the finite element model so that expression (5-45) can be computed. In the second alternative, one can either condense the stiffness matrix to the experimental mode shape DOFs or expand the experimental mode shapes to the DOFs of the finite element model matrices. The reduction of the stiffness matrix by the Guyan method is an exact operation in which none of the structural complexity is lost (Guyan, 1965) so it seems that this would be a preferable option as compared to the expansion of the mode shapes which tend to amplify the experimental errors. 
A disadvantage of this method is that it does not take the measured eigenfrequencies into account which convey useful information about the state of the structure and also tend to be measured significantly more accurately than their modal vector counterparts.

\subsection{Flexibility based damage detection}

In structural health monitoring it is normally assumed that damage results in some degradation of the stiffness of the structure. This is equivalent to assuming that damage increases the flexibility of the structure. Hence, provided that the flexibility can be estimated from the data with enough accuracy, it can be used as an indicator of damage, and also possibly to determine the location and extent of damage. This is the basis of the use of flexibility as a damage detection indicator (e.g. Toksoy and Aktan 1994; Pandey and Biswas 1994, Doebling and Farrar 1996). The modal flexibility matrix of a structure can be expressed in terms of the modal properties of the structure, namely the mass-normalized mode shapes $(\Phi)$ and the spectral matrix $(\Lambda)$, as follows

$$
\mathbf{F}_{n \times n}=\boldsymbol{\Phi} \boldsymbol{\Lambda}^{-1} \boldsymbol{\Phi}^{T}
$$

where $n$ is the number of DOFs of the structural system. Physically, the flexibility coefficient $F_{i j}$ represents the static displacements at the $i^{\text {th }}$ DOF from applying a static unit load at the $j^{\text {th }}$ DOF. In practice, usually only a limited number of vibration modes and eigenfrequencies $\left(n_{m}<<n\right)$ can be retrieved from the measured data. Furthermore the experimental mode shapes can normally only be measured at a limited number of sensor locations $\left(n_{s}<<n\right)$. Still, it is possible to write an approximate expression for the modal flexibility matrix as follows

$$
\mathbf{F}_{n_{s} \times n_{s}} \cong \boldsymbol{\Phi}_{n_{s} \times n_{m}} \boldsymbol{\Lambda}_{n_{m} \times n_{m}}^{-1} \boldsymbol{\Phi}_{n_{m} \times n_{s}}^{T}
$$


Since the flexibility contribution of the modes decreases with the square of their eigenfrequencies, the advantage of the flexibility matrix for practical application is that it can be accurately estimated using just a few lower order modes, which are normally the easiest to excite and measure. Once the flexibility matrix has been estimated using (5-47) for both the "undamaged" and "damaged" structure, the change in modal flexibilities can be computed

$$
\Delta \mathbf{F}_{n_{s} \times n_{s}}=\mathbf{F}_{n_{s} \times n_{s}}^{d}-\mathbf{F}_{n_{s} \times n_{s}}^{u}
$$

The $\mathrm{i}^{\text {th }}$ diagonal term of this matrix $\left(\Delta \mathrm{F}_{\mathrm{ii}}\right)$ represents the additional static displacement at the $i^{\text {th }}$ DOF due to a unit load applied at that DOF as a result of damage. To allow for an unbiased relative comparison of the flexibility changes at the different DOFs, the diagonal terms of this matrix can be normalized by the diagonal terms of the original undamaged flexibility matrix yielding a percentage change in modal flexibility, which has been proposed as damage indicator by Wang et al. (2000) and has shown to be effective as a damage indicator in damage simulation studies of the Tsing Ma suspension bridge. The percentage change in flexibility can be computed as follows

$$
\Delta F_{i i}(\%)=\frac{\left|F_{i i}^{d}-F_{i i}^{u}\right|}{F_{i i}^{u}}
$$

A drawback of this method is that it requires mass-normalized modes. While in forcevibration tests obtaining mass-normalized modes is relatively easy, the mass-normalization of the modes retrieved from output-only vibration data is not as direct and normally involves some assumptions or approximations. Doebling and Farrar (1996) briefly describe and compare several of the available methods proposed for the mass normalization of the mode shapes identified from output-only data, namely Guyan-Reduced Mass-Matrix Normalization, Orthogonal Procrustes Expansion, and Diagonal Mass Matrix. More recently other methods have been proposed (Bernal 2003; Schwarz and Richardson 2003; Brincker and Andersen 
2003). The first of these methods, by Bernal, has the limitation of requiring the mode shapes to be defined at all coordinates deemed necessary to describe the mass matrix, which is unrealistic in practice. In the latter two references, the proposed methods involve adding different masses to the structure during the measurement program and using the resulting shifts in the eigenfrequencies to determine the modal scaling factors. Again, this may not be practical for continuous structural health monitoring applications. In addition, there is a system condensation technique called System Equivalent Reduction Process (SEREP), proposed by O'Callahan et al. (1989), which preserves the dynamic properties of the original system. For the present study, the well known and relatively simple Guyan's reduction technique is applied (Guyan 1965).

\subsubsection{Guyan's reduction}

The system mass and stiffness matrices can be re-organized according to the DOFs to be retained (denoted by sub-index $r$ ) and those to be deleted (denoted by sub-index $d$ ) as follows

$$
\mathbf{K}=\left[\begin{array}{ll}
\mathbf{K}_{r r} & \mathbf{K}_{r d} \\
\mathbf{K}_{d r} & \mathbf{K}_{d d}
\end{array}\right], \quad \mathbf{M}=\left[\begin{array}{ll}
\mathbf{M}_{r r} & \mathbf{M}_{r d} \\
\mathbf{M}_{d r} & \mathbf{M}_{d d}
\end{array}\right]
$$

The condensed system matrices, denoted by sub-index $C$, are computed as follows (Humar, 1990)

$$
\begin{aligned}
& \mathbf{K}_{C}=\mathbf{K}_{r r}-\mathbf{K}_{r d} \mathbf{K}_{d d}^{-1} \mathbf{K}_{d r} \\
& \mathbf{M}_{C}=\mathbf{M}_{r r}-\mathbf{M}_{r d} \mathbf{K}_{d d}^{-1} \mathbf{K}_{d r}+\mathbf{K}_{r d} \mathbf{K}_{d d}^{-1} \mathbf{M}_{d d} \mathbf{K}_{d d}^{-1} \mathbf{K}_{d r}-\left(\mathbf{M}_{r d} \mathbf{K}_{d d}^{-1} \mathbf{K}_{d r}\right)^{T}
\end{aligned}
$$

Although the formulation is based on the static condensation procedure, it can be applied to situations where there are inertial and/or external forces acting on the eliminated DOFs (Humar, 1990). However, the reliability of the results depends on the proper selection of the 
retained DOFs so that the mass of the system is properly distributed in the reduced system. Typically many DOFs are still required to guarantee an accurate solution (Avitabile et al. 1989).

\subsubsection{Modal strain energy vs. flexibility approach}

A comparison of the flexibility and the modal stain energy index approaches is presented in Table 5-1.

Based on the comparison study by Humar (2005), the MSE method seems to be promising for practical use when the modal curvatures are computed from strain measurements made directly on the structure but the accuracy of the results is no longer acceptable when the curvatures need to be calculated numerically from the translational modal displacements. Currently, traditional strain gauges as well as other more advanced strain measuring devices based on fibre optics tend to be relatively delicate instruments. Therefore, for practical applications of long-term continuous monitoring systems maintaining the strain sensors in good operational condition may be more difficult and costly than the traditional translational DOF instrumentation (accelerometers, displacement meters, etc.) which tend to require relatively low maintenance. In the future, it may be expected that strain sensing technology will improve, and therefore the MSE method may become more attractive for continuous health monitoring projects. For the present time, with the above practical issues in mind, it is preferred in this study to evaluate the feasibility of applying the flexibility method for continuous monitoring instead of the modal strain energy method because of the fact that the former method relies on the translational DOFs instead of the curvatures. As noted in Table 5-1, the flexibility method offers some additional advantages versus the modal strain energy method such as: not only using the mode shape data but also the measured frequencies, which may increase the robustness of the method; and also the 
distinct tendency of the flexibility to increase with damage. In this thesis, the flexibility approach is selected and will be applied to the simulated data from the healthy and damaged models of the Confederation Bridge and the damage detection results will be compared against those obtained by the finite element updating method to establish which approach appears to be most suitable for practical implementation, to identify the potential drawbacks and advantages of each technique as well as any general difficulties. Having done this, if possible, solutions will be proposed to improve upon the existing techniques.

\subsection{Application of flexibility-based damage detection}

From the literature, the damage detection approach using the dynamically measured flexibility matrix of the structures seems to be one of the standard methods (Doebling et al. 1996). An investigation on the effectiveness of the flexibility approach for damage detection is presented here as a comparison to gauge the damage detection results obtained via the finite element updating method presented in Chapter 6. The flexibility based algorithm presented in Section 5.4 is considered in this study. As noted earlier, the flexibility based approach requires mass-normalization of the mode shapes. Here, the approach suggested by Doebling and Farrar (1996) is used for this purpose. The "measured" mode shapes, which are initially arbitrarily scaled or scaled to unity at a reference coefficient, are mass normalized using the mass matrix of the FE model, which is reduced to the measurement DOFs using Guyan's condensation technique as presented in Section 5.4. In practice, the massnormalization operation inevitably leads to some loss of accuracy. The reason is that the mass-matrix of the FE model is only an approximation to the true mass matrix of the structure. Another reason is that Guyan reduction of the mass matrix is not exact, with accuracy largely dependent on the selection of the subset of DOFs for the condensation. 
Damage scenario 1 of the Confederation Bridge, which involves a localized stiffness reduction in one of the piers of the bridge, as described in Section 6.2.4, is considered here to evaluate the effectiveness of the flexibility based method in damage detection applications. First, the flexibility method is evaluated by carrying out the damage detection under ideal conditions: i.e. without any noise in the "measured" modal properties and assuming that all the DOFs of the FE model can be "measured". In addition, it is assumed that all of the first 50 modes of the FE model are measured. The results of percentage change in flexibility are presented in Figure 5-3. The first and most important observation is that the changes in flexibility tend to be distributed over large regions of the structure rather than being restricted to the damage location. This characteristic of the changes in flexibility makes the interpretation of the corresponding results much more difficult and indirect than results of stiffness change, such as those obtained by finite element updating. Another complicating factor is that the pattern of the changes in flexibility is quite different for each of the DOF directions $(\Delta x, \Delta y, \Delta z, \theta x, \theta y, \theta z)$. In this particular example, the results for $\Delta y, \theta y, \theta z$ are good indicators of the damage location, at least better than the other DOF directions. Through careful inspection of the results, it may be possible to infer the location of the damage. But the problem is how to know which result to look at in a real "blind" damage detection scenario without knowledge of the damage location a-priori.

In typical vibration response measurement of structures, the rotational responses are not measured, and sensors are typically installed to measure structural responses at only a reduced subset of translational DOFs of the monitored structure. In this case, the results of percentage change in flexibility are restricted to only those at the monitored DOFs. Results at other non-instrumented translational DOFs would have to be obtained by interpolation, while results for rotational DOFs would be unavailable. Figure 5-4 shows the changes in 
flexibility obtained if only the 50 DOFs shown in Figure 6-9 are instrumented and measured. All other parameters are kept unchanged. From the results of Figure 5-4, which does not include interpolation of the results to non-instrumented DOFs, it is easy to observe that the patterns of change in flexibility obtained essentially have the same characteristics as the corresponding ones shown in Figure 5-3, except for some small difference in relative magnitude and that the results are restricted to the instrumented DOFs only. The restriction of the flexibility results to only at the instrumented DOFs further obscures the interpretation of the results. The need of information for the rotational DOFs, which provide the most useful information in the results shown in Figure 5-3 is also a major drawback of the flexibility method.

In light of the results, it seems the potential of the flexibility change damage detection technique is limited given that it runs into difficulties even in a highly idealized scenario without the consideration of the effect of noise in the "measured" modal properties. As a result, this technique is not considered further in this work. It should be noted however that the changes in modal flexibility do appear to have good damage sensitivity characteristics. Hence, changes in modal flexibility may have good potential for use in the objective function in FE updating techniques, as shown by the research of Jaishi and Ren (2006).

\subsubsection{Damage Locating Vectors (DLV) approach}

Recently, a technique called Damage Locating Vectors (DLVs) has been proposed by Bernal (2002) that theoretically can overcome some of the problems of the flexibility technique discussed here. Essentially, the DLV technique maps the changes in flexibility to the element domain, making it easier to pinpoint the exact damage locations. The DLV technique has shown good performance in damage detection simulations of relatively simple truss structures (Gao and Spencer 2006; Bernal 2002, 2006). However, further investigation 
is needed to confirm the applicability of this technique to other types of structures under more realistic scenarios.

The DLV approach is based on the determination of a special set of load vectors $(\mathbf{L})$ that induce no stress in the damaged elements. The basis of the method is that since these load vectors induce no stress in the damage elements, the displacement patterns of the structures under these particular load vectors are the same for the undamaged and damaged structures. This can be written as follows

$$
\mathbf{F}_{d} \mathbf{L}=\mathbf{F}_{u} \mathbf{L} \quad \text { or } \quad \mathbf{F}_{\Delta} \mathbf{L}=\left(\mathbf{F}_{d}-\mathbf{F}_{u}\right) \mathbf{L}=0
$$

Therefore, finding the set of DLVs (i.e. L) is equivalent to finding the null-space of the change in flexibility. To do that, the singular value decomposition can be employed as follows

$$
\mathbf{F}_{\Delta}=\mathbf{U S V}^{T}=\left[\begin{array}{ll}
\mathbf{U}_{1} & \mathbf{U}_{0}
\end{array}\left[\begin{array}{cc}
\mathbf{S}_{1} & \mathbf{0} \\
\mathbf{0} & \mathbf{0}
\end{array}\right]\left[\begin{array}{ll}
\mathbf{V}_{1} & \mathbf{V}_{0}
\end{array}\right]^{T}\right.
$$

Post-multiplying by orthonormal matrix V,

$$
\left[\begin{array}{ll}
\mathbf{F}_{\Delta} \mathbf{V}_{1} & \mathbf{F}_{\Delta} \mathbf{V}_{0}
\end{array}\right]=\left[\begin{array}{ll}
\mathbf{U}_{1} \mathbf{S}_{1} & \mathbf{0}
\end{array}\right]
$$

From which,

$$
\mathbf{F}_{\Delta} \mathbf{V}_{0}=\mathbf{0}
$$

Therefore the damage locating vectors are the right singular vectors of the change in flexibility matrix $\mathbf{F}_{\Delta}$. In practice none of the singular values is equal to zero and some sort of tolerance needs to be used to determine the appropriate set of right singular vectors. Once the DLVs have been computed, they are applied to an undamaged model of the structure 
and the stress in each element is calculated. The stress for beam elements can be taken as follows:

$$
\sigma_{j}=\sqrt{\left(M_{1}^{2}+M_{2}^{2}+M_{1} M_{2}\right)}
$$

where $M_{1}$ and $M_{2}$ are the moments at each end of the beam. Then, the element stresses are normalized and averaged over all of the different load cases as follows

$$
\bar{\sigma}_{j}^{N}=\sum_{i=1}^{\# D L V s}\left(\frac{\sigma_{i j}}{\max _{k}\left(\sigma_{i k}\right)}\right)
$$

where $\sigma_{i j}$ is the stress in the $\mathrm{j}$-th element induced by the $\mathrm{i}$-th DLV and $\bar{\sigma}_{j}^{N}$ is the "average" normalized stress in the $\mathrm{j}$-th element. In practice, the average normalized stress in the damage elements is typically not exactly zero due to the different approximations involved. Bernal (2006) suggests that a set of potentially damaged (PD) elements can be determined via the following approximate criterion:

$$
P D=\left\{\text { elements } \mid \bar{\sigma}_{j}^{N} \leq 0.1 \cdot \max _{j}\left(\bar{\sigma}_{j}^{N}\right)\right\}
$$

This approach is tested here by considering a damage scenario occurring in a relatively simple valley bridge structure. The test structure, shown in Figure 5-5, is relatively simple but nevertheless may be considered representative of a realistic bridge structure. As shown in Figure 5-5, the structure is not symmetric. Consequently, the modal behaviour of the structure is more complex than that of a corresponding symmetrical structure, with no purely symmetric or anti symmetric modes, as is often the case in practice. The structure's geometric configuration corresponds to what may be considered typical of a bridge crossing over a narrow mountain valley. Hence, for the sake of simplicity, this structure is referred to as the "valley bridge". The elastic modulus of the concrete is taken as $24.82 \mathrm{GPa}$. 
A damage scenario of settlement of the central pier is considered. In this damage scenario it is assumed that the settlement of the central pier will result in cracking and stiffness reduction of the girder elements 21 to 24 . Elements 21 and 24 are assumed to suffer stiffness reductions of $25 \%$, while elements 22 and 23 suffer stiffness reductions of $50 \%$. The stiffness reduction in these elements is introduced in the FE model by lowering the elastic modulus of these elements.

Again, idealized conditions are considered to preliminarily test the performance of the proposed damage locating algorithm. It is assumed that the first 25 modes of the structure can be measured, using the "sensor" locations shown in Figure 5-6. No noise is introduced in the "measured" modal parameters. Using these measured modes the approximate modal flexibility matrix is computed according to Eq. (5-47). Then, the above outlined procedure is followed. The results are shown in Figure 5-7. The corresponding set of potentially damaged elements determined via Eq. (5-7) is: $P D=\left\{\begin{array}{lllll}4 & 7 & 21 & 22 & 24\end{array}\right\}$. This set includes 3 out of the 4 damaged elements, which are 21, 22, 23 and 24 but also includes two false positive damage identifications, namely elements 4 and 7 , which are actually intact. It seems that relatively stiffer regions of the structure - in this case the piers tend to exhibit lower stresses, in some cases approaching the threshold for damage identification, which may result in a bias toward false positive identifications in these regions.

A theoretical check of the DLV method can be performed using the exact flexibility matrix, i.e. the inverse of the stiffness matrix. Using the exact flexibility matrix is equivalent to having the exact measurements of all vibration modes at all DOFs. Under such theoretical and ideal conditions the results of damage location should be accurate. The results using the exact flexibility matrix are shown in Figure 5-8. As can be observed from the figure, in the exact case the contrast between damaged and non-damaged elements is not so clear as in the 
approximate case (Figure 5-7), which means that if the damage location criterion of Eq. (5-7) is applied, the potentially damaged element set for the exact case would be empty. The reason why the stress field computed using the exact flexibility does not yield a marked distinction between damaged and non-damaged elements is not evident, but it is probably due to the contributions of the higher modes. Nonetheless, the 4 damaged elements have the lowest average normalized stress, as should be the case. This indicates that the damage locating criterion of Eq. (5-7) may not be applicable here. Given that the empirical criterion of Eq. (5-7) is developed for lower order modal approximations to the flexibility matrix, it does not necessarily apply in the present exact case. The criterion for the exact case can be redefined as follows,

$$
P D=\left\{\text { elements } \mid \bar{\sigma}_{j}^{N} \leq 0.5 \cdot \max _{j}\left(\bar{\sigma}_{j}^{N}\right)\right\}
$$

If this less stringent criterion is applied, then the identified set of potentially damaged elements would be correct, i.e. $P D=\left\{\begin{array}{llll}21 & 22 & 23 & 24\end{array}\right\}$. Thus, at least in the theoretical exact case the method seems to yield the right damage locations.

However, in practice the flexibility can only be approximately estimated from a few lower order modes. Thus, the results presented earlier show that the performance of the DLV technique, even in the simple, noise free, and highly idealized structural scenario considered is not satisfactory. While it is recognized that a more thorough evaluation is necessary to evaluate conclusively about the performance of this technique on typical bridge structures, the results obtained here provide a good preliminary indication that the applicability of the DLV technique seems to be limited for use in practical damage detection. 
Table 5-1: Comparison of flexibility and modal strain energy damage detection techniques

\begin{tabular}{|c|c|c|}
\hline & MSE damage index method & Flexibility Method \\
\hline Modal data used & $\begin{array}{l}\text { Modal curvatures, preferably obtained from strain } \\
\text { measurements }\end{array}$ & $\begin{array}{l}\text { Mode shapes and modal } \\
\text { frequencies }\end{array}$ \\
\hline Type of result & $\begin{array}{l}\text { Damage index for each finite element of the } \\
\text { model. Hence, damage is identified at the element } \\
\text { level. }\end{array}$ & $\begin{array}{l}\text { Percentage change in } \\
\text { flexibility for each DOF. } \\
\text { Damage is identified at the } \\
\text { nodal DOF level. }\end{array}$ \\
\hline $\begin{array}{l}\text { Effect of } \\
\text { damage }\end{array}$ & $\begin{array}{l}\text { The MSE is essentially stiffness times } \\
\text { displacement squared }\left(k s^{2}\right) \text { (where } k \text { represents } \\
\text { stiffness and } s \text { is displacement or rotation). While } \\
\text { stiffness }(k) \text { tends to decrease with damage the } \\
\text { corresponding displacements }(s) \text { normally tend to } \\
\text { increase with damage. Therefore it is not entirely } \\
\text { clear if the strain energy increases or decreases } \\
\text { with damage. }\end{array}$ & $\begin{array}{l}\text { Since flexibility is the } \\
\text { inverse of stiffness and } \\
\text { stiffness tends to decrease } \\
\text { with damage, then } \\
\text { flexibility tends to increase } \\
\text { with damage. }\end{array}$ \\
\hline $\begin{array}{l}\text { Other } \\
\text { Disadvantages }\end{array}$ & $\begin{array}{l}\text { When the measured data consists of translational } \\
\text { DOFs instead of strains, the modal curvatures } \\
\text { must be obtained by numerical differentiation. } \\
\text { This process significantly amplifies the } \\
\text { experimental errors and leads to inaccuracies in } \\
\text { the damage detection results. }\end{array}$ & $\begin{array}{l}\text { Mass-normalized mode } \\
\text { shapes are required, the } \\
\text { computation of which } \\
\text { involves some } \\
\text { approximations }\end{array}$ \\
\hline $\begin{array}{c}\text { Other } \\
\text { Advantages }\end{array}$ & $\begin{array}{l}\text { Any consistent mode shape normalization may be } \\
\text { used }\end{array}$ & $\begin{array}{l}\text { Has shown good } \\
\text { performance in damage } \\
\text { simulation studies of } \\
\text { Tsing Ma Bridge }\end{array}$ \\
\hline
\end{tabular}



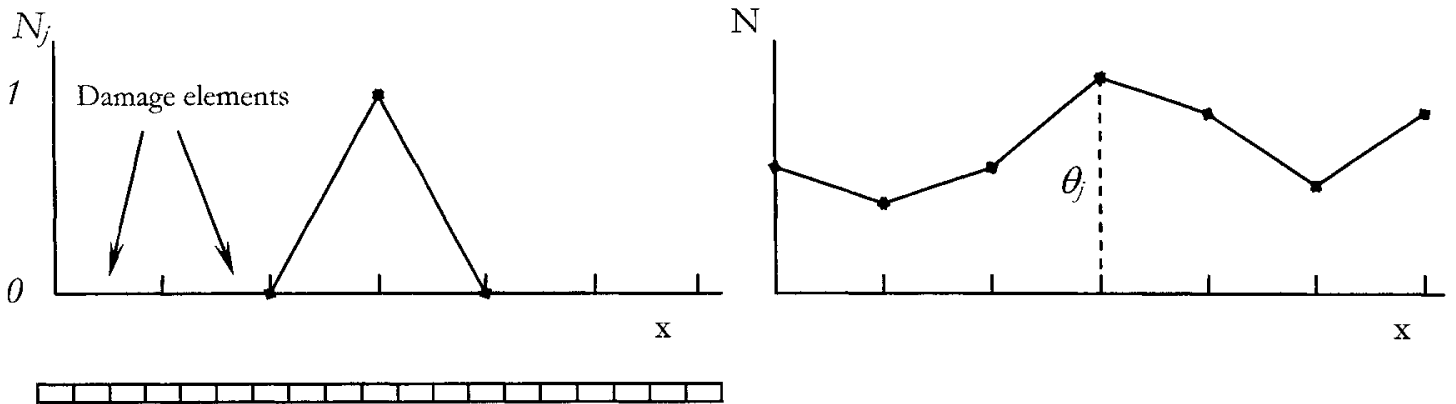

Finite element mesh

(a)

(b)

Figure 5-1: Construction of piece-wise linear damage functions over finite element mesh. (a) Local damage shape function, (b) global damage shape function. 
"FEUPDATE"

Definition of problem parameters including:

- Reference FE model input file

- Damage element mesh

- 'Measured' modes and DOFs (of 'damaged' structure)

- Contamination of 'measured' modes with simulated variability

- Reference DOFs for mode normalization

- Weighting of residuals

- Number of FE modes to be used for theoretical sensitivity computation

- Optimization parameters for nonlinear least squares trust-region algorithm

$\begin{array}{ll}\circ & \text { Starting values of optimization variables } \\ \circ & \text { Bound constraints for optimization variables } \\ \circ & \text { Convergence criteria }\end{array}$

'LSQNONLIN"

Trust-region Gauss-Newton optimization of non-linear least squares function via built-in Matlab optimization function.

- Iteratively updates the vector of optimization variables based on the value of the objective function and its Jacobian

- Stops when convergence criteria are satisfied. (Namely: the objective function value over successive iterations is changing by less than a given tolerance value, or the optimization variables are changing by less than a given tolerance value)

\section{"OBJFUN"}

- Evaluation of value of objective function value and computation of Jacobian. Essential tasks include:

- Mapping of optimization variables to element correction factors

- Generate input of updated FE file based on the above correction factors

- Call FE package to run modal analysis for updated model

- Match 'measured' modes with those from updated model

- Computed residual vector

- Compute Jacobian as per analytical expressions by Fox and Kapoor (1968)

Figure 5-2: Flow chart of subroutines of implemented FE updating damage detection algorithm for use in damage detection simulation study 

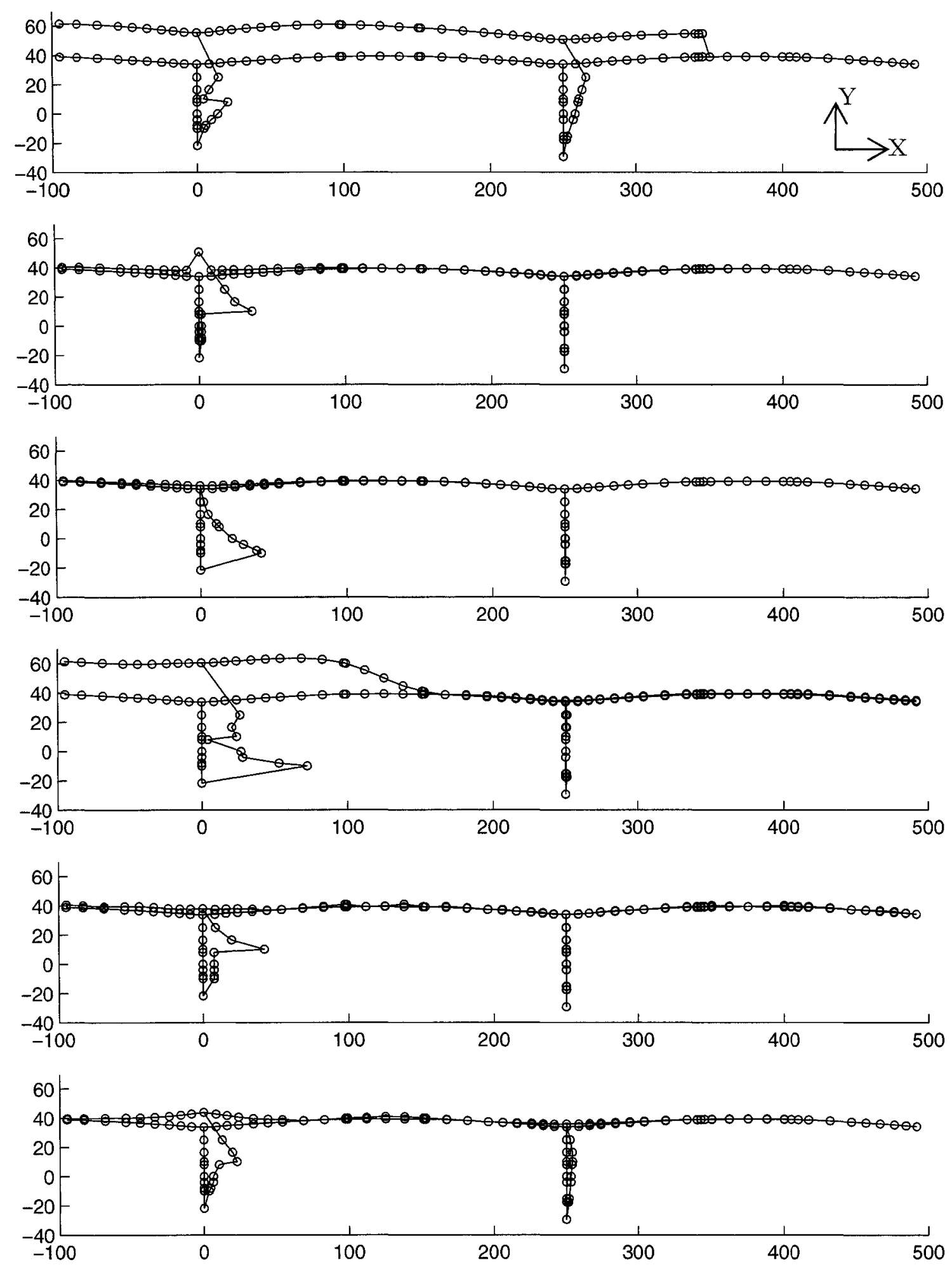

Figure 5-3: Percentage change in flexibility (from Eq. (5-49)) for each of the 6 DOF directions $(\Delta x, \Delta y$, $\Delta z, \theta x, \theta y, \theta z)$, in corresponding order from top to bottom. Assuming all FE DOFs could be instrumented. Amplification factors: $[10,1,0.3,1,1,2.5]$; 

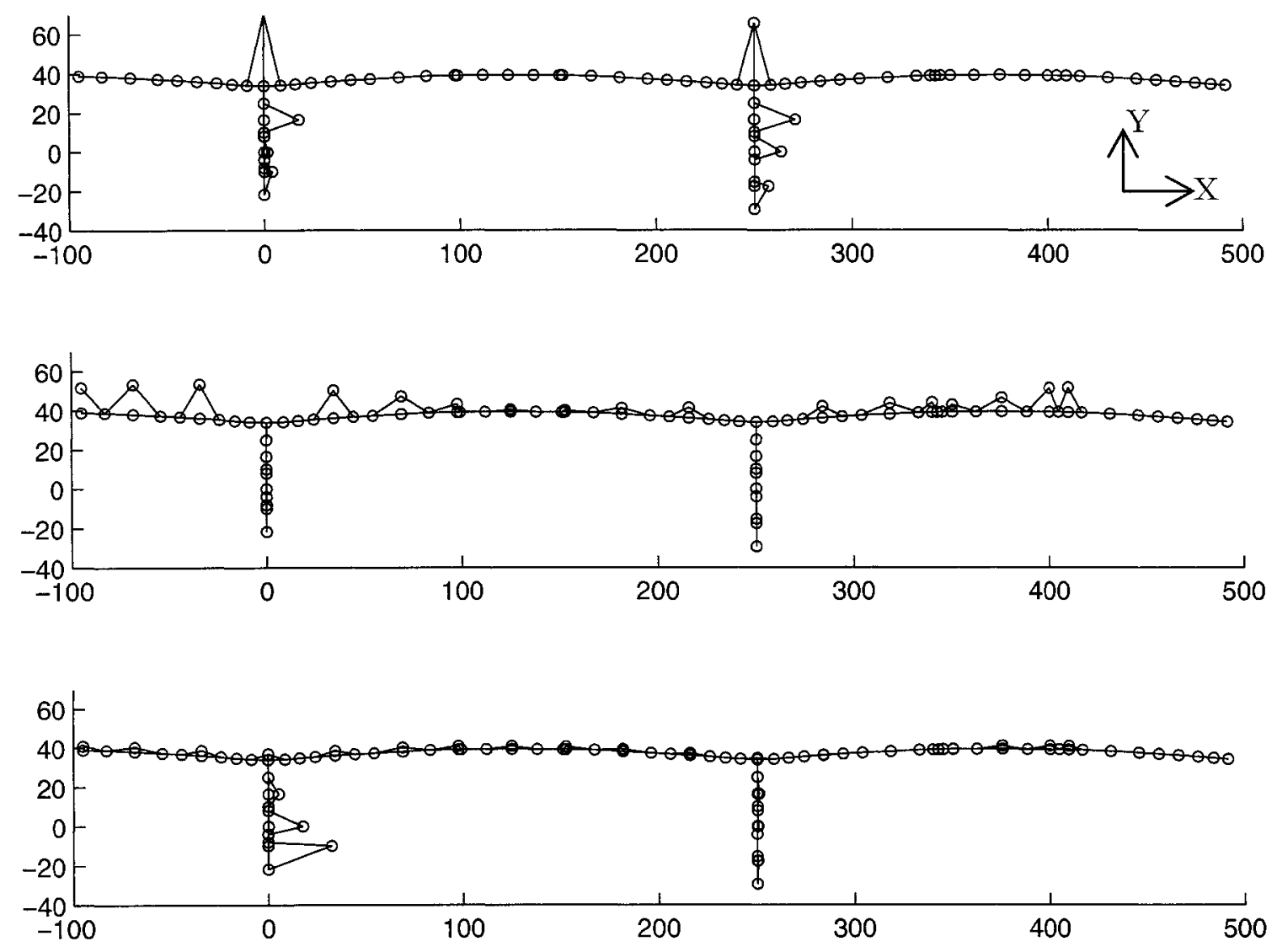

Figure 5-4: Percentage change in flexibility (from Eq. (5-49)) for each of the 3 measured DOF directions $(\Delta x, \Delta y, \Delta z)$, in corresponding order from top to bottom, assuming instrumented DOFs are only those shown in Figure 6-9. Note: No interpolation carried out for non-instrumented DOFs. Amplification factors: $[7.5,3,0.3]$; 


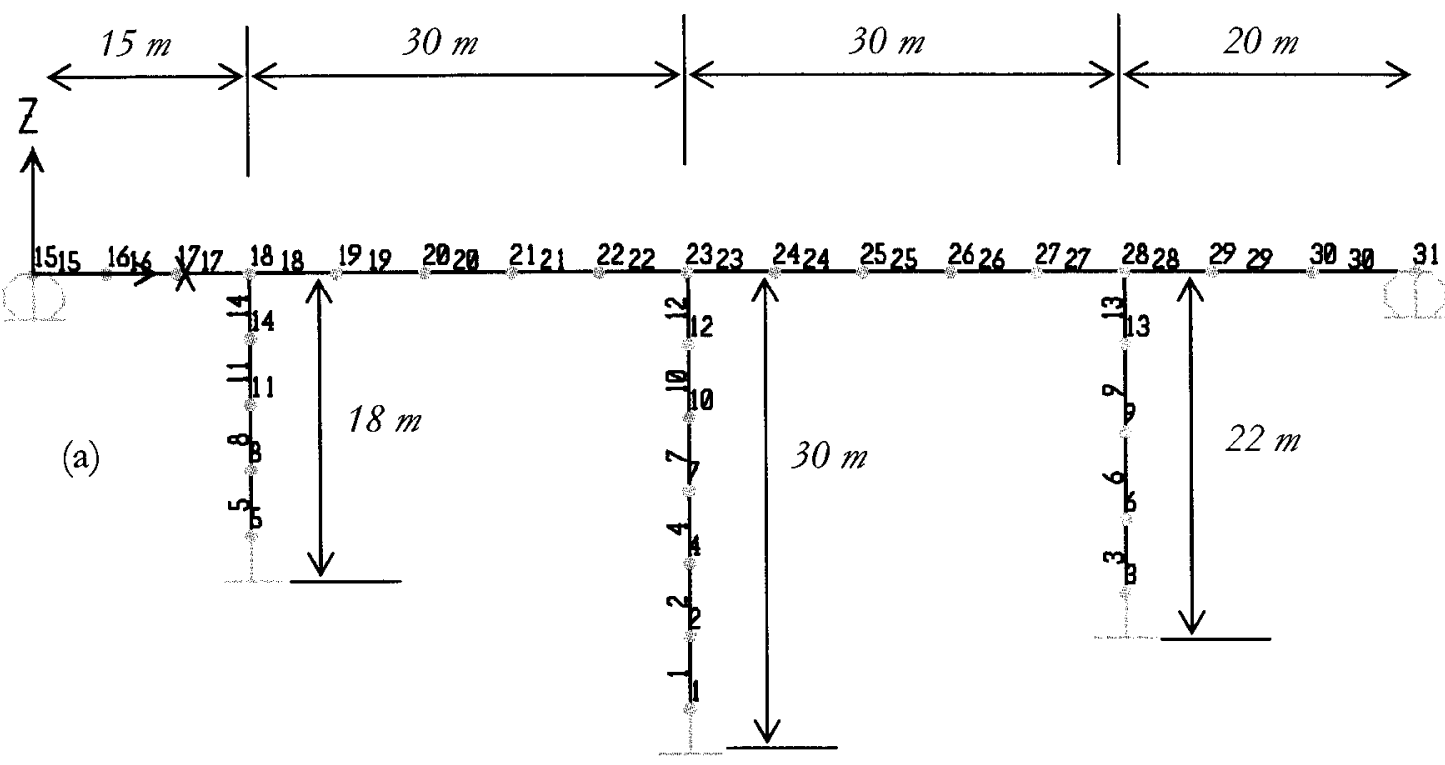

(b)
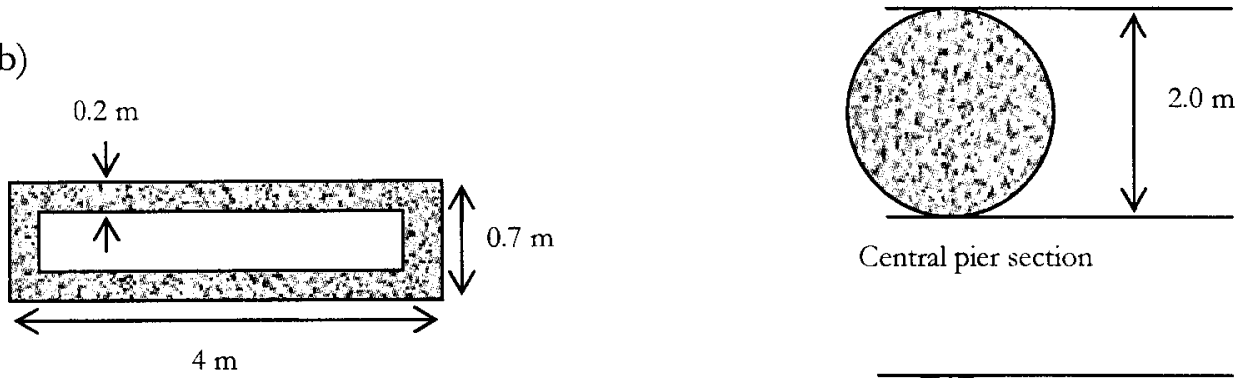

Central pier section

Girder cross section

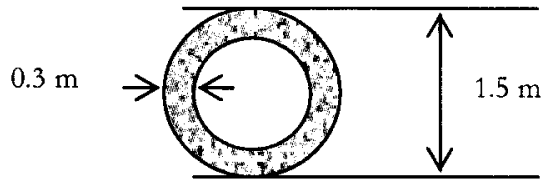

Side pier section

Figure 5-5: Simple valley bridge; (a) Element and joint numbers of finite element model; (b) member cross sections. 


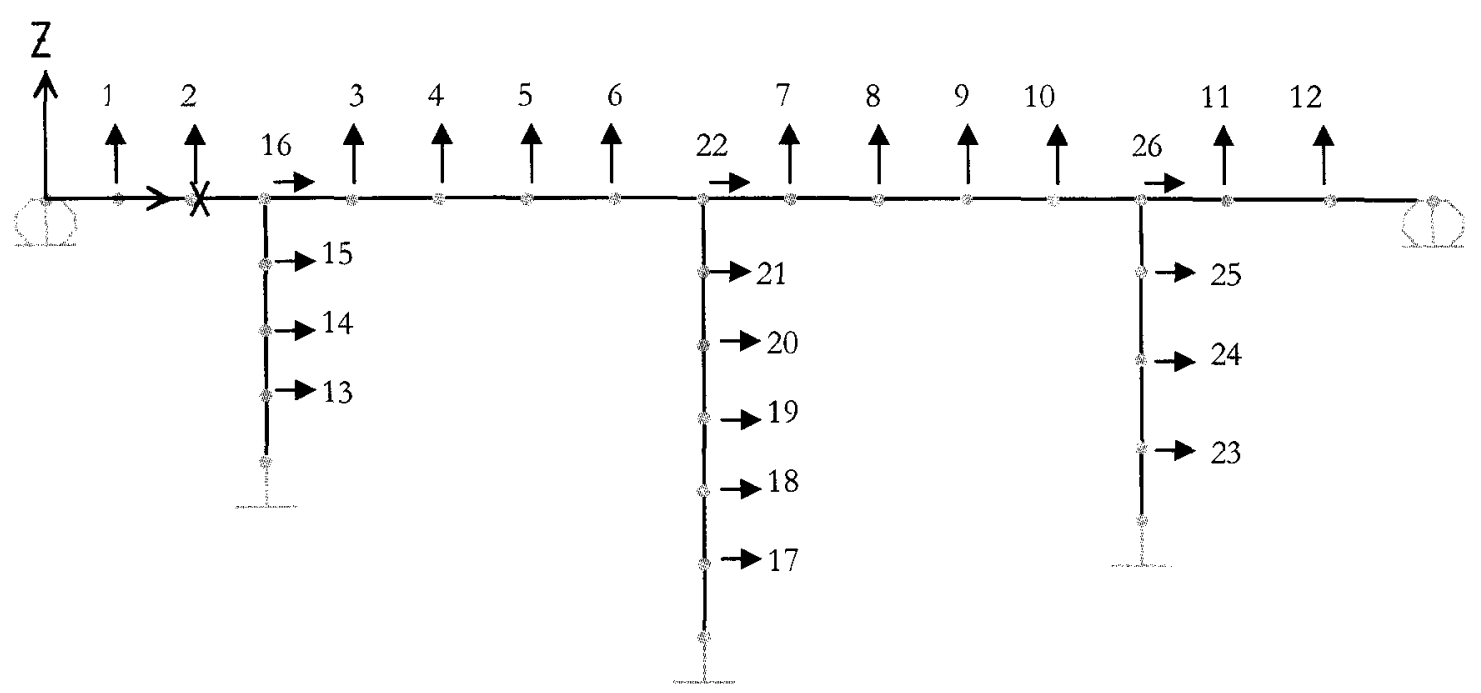

Figure 5-6: "Measured" DOFs for the valley bridge. 


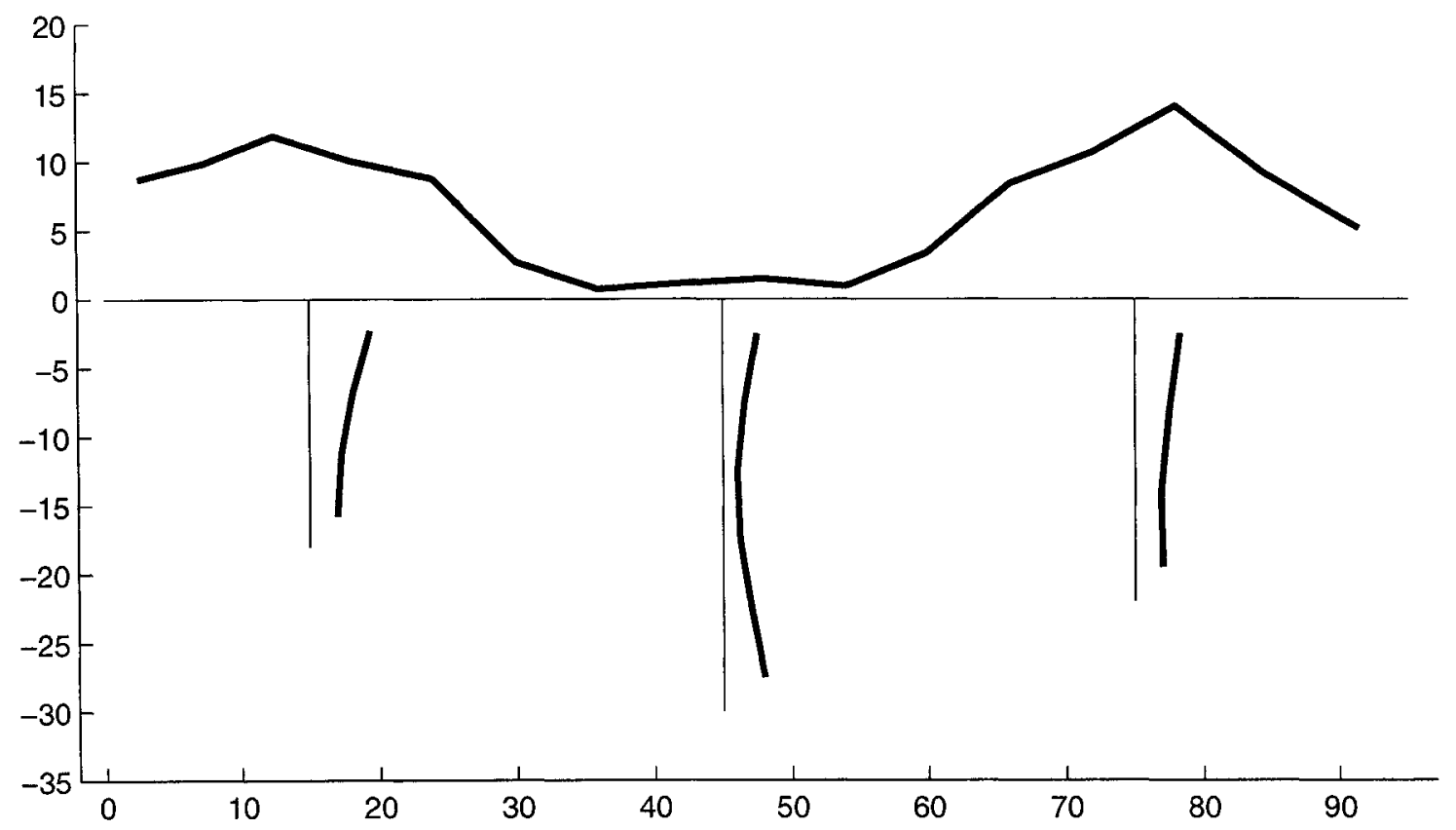

Figure 5-7: Average normalized element stress field obtained via DLV approach for damage scenario 1 of the "valley" bridge example. Regions of near-zero stress are indicative of damage. An amplification factor of 2 is used for the plot. 


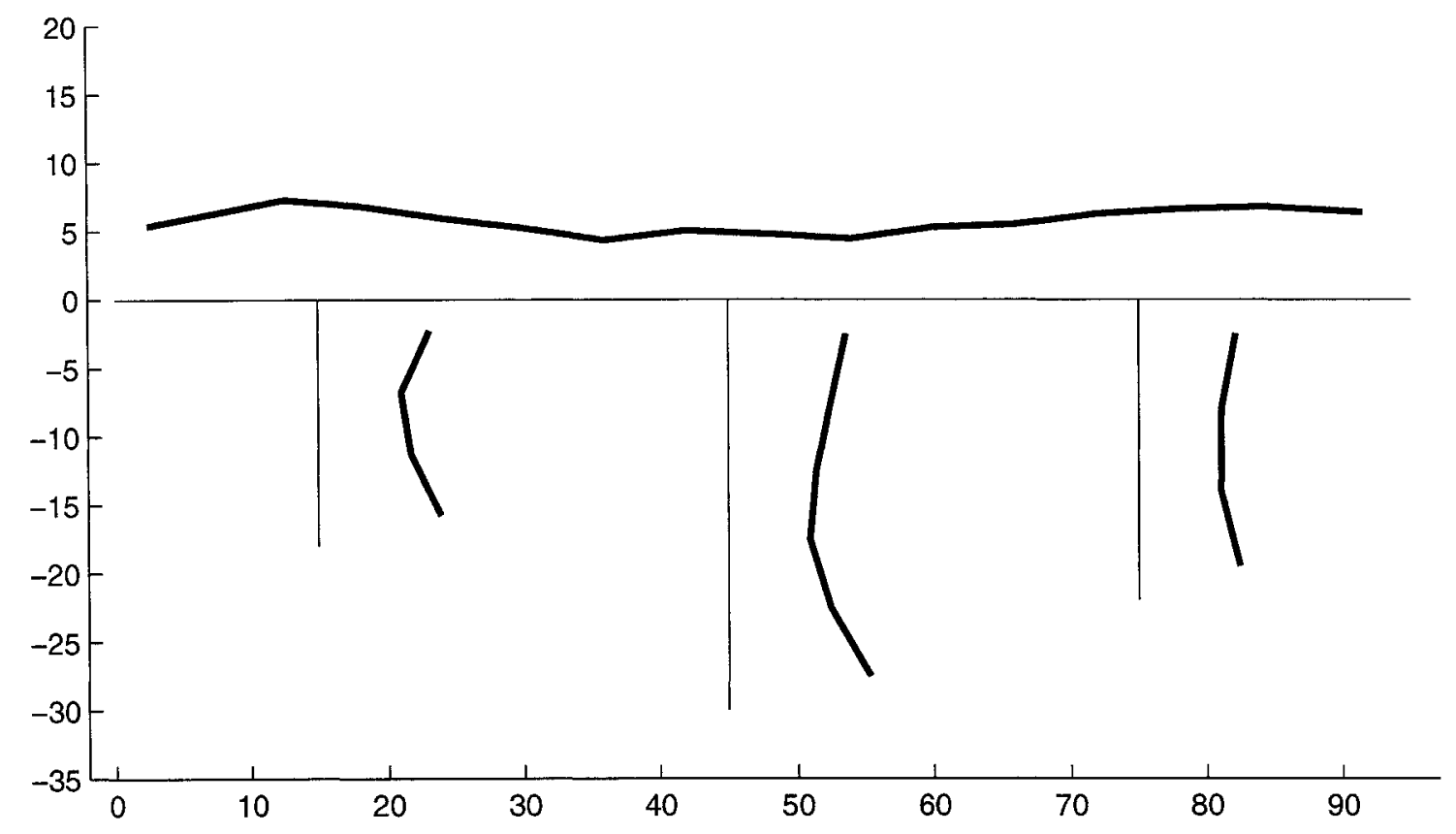

Figure 5-8: Average normalized element stress field obtained via DLV approach for damage scenario 1 of the "valley" bridge example using exact flexibility matrix. An amplification factor of 0.3 is used for the plot. 


\section{Chapter 6. Application of finite element updating damage detection}

\subsection{Damage detection of a simple bridge}

In this chapter the feasibility of applying vibration-based damage detection techniques in practice is investigated. The evaluation is based on using the field-monitoring results and findings presented in Chapters 2 and 3 of this thesis. Prior to applying the finite element updating damage detection method to the more complex model of the Confederation Bridge, a relatively simple $2 \mathrm{D}$ model of a hypothetical bridge structure is used herein for verification of the of the finite element updating damage detection algorithm implementation.

\subsubsection{Structure and FE model}

The test structure, shown in Figure 5-5, is relatively simple but nevertheless may be considered representative of a realistic bridge structure with a variety of structural layout characteristics. As such, it can be used as a good example to assess the ability of the FE updating algorithm to detect damage by health monitoring of bridge structures. As shown in Figure 5-5, the structure is not symmetric. Consequently, the modal behaviour of the structure is more complex than that of a corresponding symmetrical structure, with no purely symmetric or anti symmetric modes, as is often the case in practice. The structure's geometric configuration corresponds to what may be considered typical of a bridge crossing over a narrow mountain valley. Hence, for the sake of simplicity, hereinforth this structure is referred to as the "valley bridge". The elastic modulus of the concrete is taken as $24.82 \mathrm{GPa}$. 


\subsubsection{Damage scenarios}

\section{Scenario 1: Settlement of central pier}

In this damage scenario it is assumed that the settlement of the central pier will result in cracking and stiffness reduction of the girder elements 21 to 24 . Elements 21 and 24 are assumed to suffer stiffness reductions of $25 \%$, while elements 22 and 23 suffer stiffness reductions of $50 \%$. The stiffness reduction in these elements is introduced in the FE model by lowering the elastic modulus of these elements.

\section{Scenario 2: Damage of side pier}

In this damage scenario, it is assumed that the shortest left pier of the bridge model suffers damage at the top and bottom portions corresponding to elements 5 and 14 as a likely damage pattern of the bridge during severe earthquakes. The assumed stiffness reduction in each of these two elements is $50 \%$. This damage scenario represents the typical kind of damage resulting from earthquake loading.

\section{Scenario 3: Damage at mid-span of the girder (element 25)}

In this scenario, element 25 is assumed to suffer a $50 \%$ stiffness reduction. It is noted that this discrete damage pattern cannot be identified exactly by the piecewise linear damage function mesh of Figure 6-1. This scenario is used to verify the ability of the method to identify discrete damage that cannot be exactly matched by the piece-wise damage function mesh. The FE updating method is still expected to converge on average to the best possible representation of the actual damage by the distributed damage functions.

\subsubsection{Data simulation}

The vibration measurements from a real structure typically consist of translational acceleration time-histories taken at different points on the structure. Dynamic rotational response measurements are normally not available. The acceleration data are processed and 
analyzed by system identification techniques to obtain the structural modal parameters of eigenfrequencies and mode shapes. Due to economic limitations the number of sensors and thus number of measured DOFs is normally much lower than the number of DOFs of the FE model. Furthermore, normally only a small subset of the total number of structural vibration modes can be well excited and accurately retrieved from the vibration data, especially when using vibration responses under typical operational excitations. To reflect these conditions, only a reduced set of modes and DOFs are used in the damage detection simulation study. Mode shape displacements taken from the finite element model are "measured" only at a limited number of locations corresponding to selected translational DOFs.

The variability of modal frequencies and mode shape coefficients is modeled according to the formulations presented in Section 3.6.2. In the simulation of the variability of the measured modal properties, it is assumed that the measured modal properties of both the damaged and undamaged structures are average values obtained from samples of sizes $N$, $=N_{2}=10$ (in Eq. 3-16).

For the valley bridge, 26 translational DOfs are measured, as shown in Figure 5-6, and two different sets of measured modes are considered (Figure 6-2):

1. FE reference model modes 1 to 8 . This results in $8 \times 25=200$ mode shape residuals (reference DOF residual is excluded)

2. FE reference model modes 2, 3, 5, 8. Note that this mode set is perhaps more realistic than set 1 . This results in 100 mode shape residuals.

\subsubsection{FE updating parameters}

The elastic modulus of the elements of the FE model is updated by the optimization algorithm of minimizing the residuals or discrepancies between the measured properties 
from the damaged structure and those of the updated FE model. It is assumed that identified reductions in the stiffness of the elements will indicate zones of damage. The updating variables are the multiplication factors of a piecewise linear damage function, as shown in Figure 6-1. This function gives the variation of the element correction factors throughout the model and it is used to reduced the number of optimization variables and improve the condition of the optimization problem.

\subsubsection{Objective function}

The function to be minimized is the 2-norm squared (or sum-of squares) of the residual vector, as expressed in Eq. (5-1), which contains both the eigenfrequency and modeshape coefficient residuals. As shown in Eq. (5-2), weighting factors of the residuals can be used to give more importance to selected residuals. For example, more weight can be given to those residuals having the least measurement uncertainty. Here, accordingly, weighting is used to increase the relative importance of the eigenfrequency residuals. For this purpose, the mode shape residuals carry a weight of $w_{s}=1 / 3$ while the eigenfrequencies carry $w_{f}=1$.

In the computation of the mode shape sensitivities, the first 25 mode shapes of the FE model are considered for Eq. (5-24). The higher the number of modes used in computation of the sensitivities, the more accurate they will be. Higher accuracy leads in turn to improved condition of the Jacobian matrix and the optimization problem in general. However, the contribution of each mode decreases with the mode number. Considering this, a balance between accuracy and computation efficiency should be sought.

Other relevant problem parameters include:

- Initial values of optimization variables $\left(\theta_{0}\right)$ : uniformly distributed random numbers in the interval $(-0.1,0.1)$. 
- Constraints on optimization variables $\theta$ : lower bound values of -1 , upper bound +1 .

- Convergence criteria: Convergence is achieved when the objective function is changing by less than $10^{-5}$, or the norm of the step taken is less than $10^{-5}$.

\subsubsection{Damage detection results without variability Damage scenario 1 - mode set 1}

Figure 6-3(a) shows the identified correction factors distribution for 5 simulations of the optimization algorithm using different random starting points for the optimization variables using FE modes 1 to 8 as "measured modes". All 5 simulations converge to the correct solution. The condition number of the Jacobian Matrix at the solution is 125 for all 5 simulations.

\section{Damage scenario 1 - mode set 2}

Figure 6-3(b) shows the results for 5 simulations of the FE updating algorithm using only FE modes 2, 3, 5 and 8 as "measured modes". The condition number of the Jacobian matrix at the solution is 454 for all 5 simulations, which is higher than for the case of using 8 measured modes. However, the damage is still identified properly and consistently.

\section{Damage scenario 2 - mode set 1}

Figure 6-4(a) shows the results obtained for the damage scenario of damage at the top and bottom of the left shortest pier. Damage is identified accurately independent of the starting point used showing that the method is accurate as expected. The condition number of the Jacobian at the solution is 75 .

\section{Damage scenario 2 - mode set 2}

Figure 6-4(b) shows the results obtained for the damage scenario of damage at the top and bottom of the left pier using a reduced measured mode set. Again, damage is 
identified accurately regardless of the starting point showing that the method is working as expected. The condition number of the Jacobian at the solution is 253 .

\section{Damage scenario 3 - mode set 1}

Figure 6-5(a) shows the results of 5 simulations of the FE updating damage detection algorithm for damage scenario 3 using FE modes 1 to 8 as "measured modes" and the damage functions of Figure 6-1. The condition number of the Jacobian matrix at the solution is 152 for all 5 simulations. It is observed that the solution reached is stable but shows considerable deviation from the actual damage. From the identified damage pattern of Figure 6-5(a), it would seem that the bridge has undergone stiffness changes not only in the girder but also in the piers, which is not the case. In practice this would be a highly misleading result. Although it is not possible that the actual damage be matched exactly by the damage function mesh of Figure 6-1, one would at least expect the result to be close to the best possible representation of the actual damage by the distributed damage functions. A linear least squares fit, representing the "best possible" fit of the actual damage by the distributed damage function, is shown for comparison in Figure 6-5(a). The least squares fit also deviates somewhat from the actual damage but it clearly indicates damage has occurred only in the girder and not in the piers.

The convergence to the "wrong" solution stems from the fact that the objective function value, i.e. sum of squared residuals of Eq. (5-2), for the FE updating solution is actually lower than the function value corresponding to the least-squares fit of the damage function to the actual damage. The sum of squared residuals for the FE updating solution is 0.0206 while the corresponding value for the least squares fit to the actual damage is 0.1506 . This example exposes an important shortcoming of the FE updating method. Whenever the actual damage pattern is highly localized, as in the present example, a relatively coarse 
damage function will not be able to match it closely and hence the algorithm might end up finding a mathematical minimum which actually differs from the best fit to the actual damage. The natural option to overcome this problem would be to refine the damage function mesh. Although this is a valid option, one cannot usually go too far in the refinement because doing this tends to lead to ill-conditioning problems. The reason for the ill-conditioning is that as the damage elements get smaller, the columns of the Jacobian matrix tend to become closer to linearly dependent. In general, it can be argued the FE updating method using damage functions seems to have serious problems in locating damage that is highly localized.

To find the real damage distribution of damage scenario 3 it would be necessary to run the damage detection with a more refined mesh. The mesh may be refined as long as the condition of the sensitivity matrix (or Jacobian) remains within a reasonable range.

In case the damage location is roughly known or suspected a priori, then the damage element mesh can be refined exclusively in the region of suspected damage to evaluate more precisely the location and severity of the damage. The damage function mesh of Figure 6-6, which targets the girder affected by damage scenario 3 , illustrates this idea.

Figure 6-5(b) shows the identified damage pattern for damage scenario 3 using the refined damage element mesh of Figure 6-6. The damage is identified correctly for all 5 independent simulations, which have different starting points. The condition number of the Jacobian at the solution is 112 .

\subsubsection{Damage detection results with variability Damage scenario 1 - mode set 1}

Figure 6-7(a) shows the results for 5 simulations of the FE updating algorithm using FE modes 1 to 8 as "measured modes". The variability of modal frequencies and mode shape coefficients is modeled according to the formulations presented in Section 3.6.2. Since 
the eigenfrequencies exhibit less variability than the mode shapes, the weight given to the eigenfrequency residuals is increased by applying weighting factors of $w_{S}=1 / 5$ to the mode shape residuals. Each simulation has a different random starting point for the updating variables $\theta$, while the realization of the random noise contaminating the "measured" mode shapes and eigenfrequencies is kept fixed, as would be the case in practice. ${ }^{5}$ The different simulations all converge to a unique minimum which deviates significantly from the actual damage pattern, especially in the stiffer regions of the structure (namely the piers), which tend to have higher noise-to-signal ratios in the measured mode shapes as a result of lower modal amplitudes. The results show that the variability of the measured eigenfrequencies and modes shapes is inevitably reflected in the damage detection output. From a practical perspective the results are not acceptable since they could lead to erroneous conclusions about the state of the structure.

One way to improve the damage identification results would be simply to use more measurements of the structural modal properties. It should be noted that for the case of a continuously monitored structure there would probably be no major inconvenient in doing this, and consequently it should be considered as a fully valid practical option. In this case, multiple samples of measured modal properties can be collected for both the "undamaged" and "damaged" structures (one sample consists of multiple measurements). The advantage is that the mean measured modal properties obtained from each sample would have different "realizations" of the measurement noise. This implies that the identified damage pattern

\footnotetext{
5 The same realization or sequence of random numbers can be obtained in Matlab by resetting the 'state' of the random number generator on every simulation. In contrast, to obtain different realizations for the starting values of the updating variables $\theta$, the computer clock is used to set the state of the generator to a different number every time.
} 
obtained from each pair of "undamaged"-"damaged" samples would have a different error. Therefore, averaging the identified damage patterns would tend to reduce the random error components, yielding a more accurate result. This is illustrated by the results shown in Figures 6-9(b) and (c). These results correspond to a second set of 5 simulations of the FE updating algorithm in which, as opposed to the simulations of Figure 6-7(a), each simulation has a different realization of the random noise in the "measured" modal properties, as well as different random starting points for the updating variables $\theta$. This is meant to reflect what would occur if different independent samples of measured modal properties could be taken and used for the damage detection, as explained above. The results show that while each independent simulation exhibits significant deviation from the actual damage pattern, especially in the relatively stiff regions of the structure, the mean identified damage pattern is reasonably close to the actual damage pattern, much more so than the single-sample result of Figure 6-7(a). This confirms that increasing the amount of measurements, by using multiple samples of multiple measurements, may be an effective way of reducing the effects of noise on the damage detection results.

\subsection{Confederation Bridge damage detection}

\subsubsection{Structure and FE model}

Figure 6-8 shows the structural layout of the Confederation Bridge, as well as the finite element model of the Confederation Bridge used in the damage detection simulations. The model, built in the SAP2000 finite element software, consists of a total of 123 beam elements and is built from the design drawing specifications. This is essentially the same finite element model as used in Chapters 2 and 3 for the verification of the expected dynamic behaviour of the structure, except for some minor refinement of the mesh by adding some 
elements in the critical regions of the structure to be damaged and also some additional nodes to obtain the modal displacements at the desired "sensor" locations.

As discussed in Chapter 2, the dynamic behaviour of the model has been found to correlate well with the actual monitored behaviour (Londoño and Lau 2003).

\subsubsection{Data simulation}

Figure 6-9 shows the sensor locations or "measurement" DOFs. A total of 50 DOFs are "measured". The subset of vibration modes from the finite element model used as "measured" modes in the damage detection study are selected on the basis that these are the experimentally extracted modes most consistently retrievable from the field monitoring data, as listed in Table 2-2 of Chapter 2. The criterion is to use only those modes that are wellexcited on a regular basis by the typical operational loadings at the bridge site. For this study, modes identified from at least a third of the available datasets are considered to meet this criterion. Figure 6-10 shows the vibration modes used for damage detection. The total of 10 modes used for FE updating results in 500 updating residuals in the objective function. All 10 modes are vertical bending vibration modes as a result of traffic being the dominant operational loading, except for the first measured mode, which is in the lateral direction.

The 10 "measured" modes and the corresponding modal frequencies are contaminated with noise according to the noise model of Eq. (3-17). Sample sizes $N_{1}$ and $N_{2}$ are taken equal to 10 in Eq. (3-15). As mentioned earlier, the amplitude dependent mode shape variability is taken into account.

\subsubsection{FE updating parameters}

Figure 6-11 shows the damage element mesh used in the damage detection simulations. The structure is divided into 21 damage elements. The damage element mesh 
reduces the number of updating variables significantly from 123 , which is the number of elements of the FE model, to just 22. The selected damage element and damage function grid is regularly and evenly distributed throughout the instrumented portion of the structure and does not favour the damage detection at any particular location. This damage element layout is meant to represent the kind of layout that could be used in practice with no a-priori knowledge of damage location.

For the computation of the sensitivities of the mode shape residuals to the updating variables, a basis of the first $75 \mathrm{FE}$ model modes is used in Eq. (5-24). . The higher the number of modes used, the more distinguishable the effect of changing the stiffness of each individual element becomes, thus improving the condition of the Jacobian matrix of the optimization problem. At the same time, the contribution of the vibration modes to the sensitivity of the residuals decreases with the mode order, so taking too many modes will lead to added computation time with no significant gain in accuracy. By taking the first 75 vibration modes of the Confederation Bridge FE model, the dynamic behaviour of the bridge is covered up to a modal frequency of $13.5 \mathrm{~Hz}$, which exceeds the frequencies of the "measured" vibration modes considered in the residual vector (the frequency of highest "measured" mode is $5 \mathrm{~Hz}$ ). Thus, 75 modes can be reasonably expected to provide a high enough accuracy while economizing computation time.

Other relevant problem parameters include:

- Initial values of optimization variables $\left(\theta_{0}\right)$ : uniformly distributed random numbers in the interval $(-0.1,0.1)$.

- Constraints on optimization variables $\theta$. lower bound values of -1 , upper bound +1 .

- Convergence criteria: the tolerance of the objective function is taken as $10^{-8}$, while the tolerance on the norm of the iteration step taken is $10^{-5}$. 
- Weighting of residuals: eigenfrequency residual weights $\left(w_{j}\right)$ are taken as $w_{f}=1$; Since mode shapes have higher errors, a lower uniform weight value is used. A constant value is used for all the mode shape residuals $\left(w_{j}\right)$. In the damage detection simulation study, the value of $w_{s}=1,1 / 3,1 / 5$ or $1 / 10$ is considered for each damage scenario.

\subsubsection{Damage scenarios}

\section{Damage scenario 1: Localized pier damage}

This damage scenario involves localized stiffness reduction in a region of the pier located in the vicinity of an abrupt change of the pier 32 cross-section, as shown in Figure 6-12. The damage is modeled by reducing the stiffness of the beam element located just above the indicated cross section by $50 \%$, the length of which is $2-\mathrm{m}$.

\section{Scenario 2: Damage at girder joint}

This damage scenario involves a stiffness reduction of $50 \%$ at one of the joints of the continuous span between piers 31 and 32 of Confederation Bridge, as shown in Figure 6-13. It should be noted that localized damage scenarios, such as the above, cannot be matched exactly by the damage element mesh to be used for the FE updating damage detection shown in Figure 6-11. The resolution of the damage detection results is limited by the size of the damage elements. To improve the resolution, the size of the elements can be reduced but there is a trade-off effect with the numerical condition of the associated optimization problem. As the damage elements become smaller, the sensitivity matrix (i.e. the Jacobian) becomes closer to singular. Therefore, a compromise must be made when designing the damage element mesh.

Inertia reductions from cracking of the cross-sections at these joints could occur if there is significant rupturing of the pre-stressing tendons than give continuity to the joint. Although significant failures of the tendons may be relatively unlikely at the early stages of 
service life, especially for a structure with such stringent design criteria and quality control as the Confederation Bridge, advanced rupturing of pre-stressing steel has been observed in older bridges and it may become a serious possibility at later service stages. For example, Vogel \& Fricker (2005) report a high rate of breakage of bonded pre-stressing wires on a 53 year old Swiss bridge, which was decommissioned based on their monitoring findings.

\section{Scenario 3: Deterioration of piers}

Civil engineering structures in and around marine environments are subjected to the simultaneous action of various deteriorating factors which can work together to significantly affect the long term performance of the structures. The deteriorating factors in cold weather marine environments include: chemical action of seawater constituents (especially $\mathrm{Cl}^{-} \mathrm{Mg}^{2+}$, $\mathrm{SO}_{4}^{2-}$, dissolved $\mathrm{CO}_{2}$ ), biochemical action from marine growth (bacteria, algae, etc.), crystallization pressure of salts within the concrete, freeze-thaw cycles, wet-dry cycles, erosion from the shocks and abrasive actions of waves and ice flows, corrosion of embedded steel, and alkali-aggregate expansion if reactive aggregates are present (Mehta 1986; Woods 1984; Lewis \& Mercer 1984). The tidal zone is normally the worst zone for concrete deterioration since it tends to involve all of the deleterious factors at the same time. Nevertheless, the parts of the structure above and below the tidal zone may still suffer significant degradation. The main factors above the tidal zone tend to be freeze-thaw action and corrosion of the embedded steel, while the submerged part is exposed mainly to chemical reactions with seawater constituents, and biochemical actions from the different marine growths.

The environmental conditions for the Confederation Bridge include temperatures from $-35^{\circ} \mathrm{C}$ to $+35^{\circ} \mathrm{C}$, drifting ice flows driven by strong local tidal currents which can result in each pier encountering approximately $1000 \mathrm{~km}$ of ice $/$ month, humid winds saturated with 
salt at maximum speeds in excess of $100 \mathrm{~km} / \mathrm{h}$ and waves up to $4.5 \mathrm{~m}$ (Aitcin 2002, Brown 2000, Anglin et al. 1997). To counter these adverse actions and satisfy the 100 -year durability design requirement, the designers of the Confederation Bridge used specially formulated high-performance concretes, which included the following characteristics: air entrainment for freeze-thaw resistance meeting CSA-A23.1:1994 requirements, higher than normal concrete cover of $75 \mathrm{~mm}$ (normally $50 \mathrm{~mm}$ for structures exposed to severe weather), high strength abrasion resistant concrete (70-100 $\mathrm{MPa}$ depending on structural component) made from low-alkali silica fume cement with pulverized fly ash (pfa), and a relatively small maximum aggregate size $(20 \mathrm{~mm})$ to ensure proper flow into densely reinforced areas (Aitcin 2002). Of these protective measures, the use of silica fume cement and the addition of fly ash should be highlighted because they lead to low concrete permeability which is the number one key factor for the long-term protection against the various degradation agents (CPCA, 1995, Mehta 1986).

Despite the special preventive measures, due to the formidably harsh conditions of the cold-weather marine environment where the Confederation Bridge stands, some deterioration over the long-term cannot be fully discarded and should be carefully monitored to ensure the structure can fulfill its 100 -year service requirement.

Compared to the damage scenarios traditionally considered in damage detection studies where only a very localized part of the structure is considered to suffer damage (e.g. reduction of a given cross-sectional area, cutting of flanges, etc), the pattern of stiffness reduction resulting from long-term exposure to the operating environment may be significantly different. In the latter case, the stiffness reduction pattern may be distributed throughout the structure, as opposed to localized, with stiffness reductions depending on the degree and type of exposure. In bridges built over water, some more heavily exposed 
regions, such as the tidal zone, may suffer more significant strength and stiffness decay over time than other regions of the structure. In addition to being distributed, the other important difference is that deterioration tends to be a relatively slow and gradual process. This characteristic may make it more difficult to detect this type of damage since it does not produce sudden changes in the vibration responses.

The ability to detect distributed degradation is evaluated here by considering a damage scenario with a $10 \%$ uniform stiffness reduction of the piers of the Confederation Bridge. In the damage simulation, the stiffness of all 4 piers included in the finite element model (Figure 6-8) is reduced by the same amount.

\subsubsection{Damage detection results without variability Damage Scenario 1: Localized pier damage}

Figure 6-14(a) shows the results of the FE updating damage detection algorithm for damage scenario 1 , i.e. a localized $50 \%$ stiffness reduction in pier 32 , without any noise introduced in the "measured" modal properties. Results are presented for 5 different starting points of the optimization variables. The results show good convergence of the algorithm, with all 5 simulations converging to the same solution. The identified damage pattern provides a clear indication of the location of damage in Pier 32. The discrepancy between the identified damage pattern and the actual damage, which is more localized, is due to the fact that it is impossible for any linear combination of the damage functions of Figure 6-11 to match the actual damage pattern. This inevitably leads to some error in the estimated damage severity and "leakage" of the identified damage to adjacent regions in the identification of the damage location. Despite this problem, from an engineering standpoint the identified damage pattern provides a useful and accurate indication of the existence of damage and its location. 


\section{Scenario 2: Damage at girder joint}

Figure 6-15(a) shows the identified damage patterns from 5 simulations of the FE updating damage detection algorithm for the damage scenario of $50 \%$ stiffness reduction in one of the cast-in-place continuous joints of the girder, without considering variability in the measured modal properties. The five simulations have different starting values for the updating variables. After trying different weights for the residuals of the objective function, it is observed that the best results without variability are obtained for a weighting factor $w_{f}=$ $w_{s}=1$, i.e. no weighting for the mode shape residuals. The actual damage pattern and the target solution or "best possible" fit of the damage function to the actual damage, obtained using linear least squares fit, are presented for comparison in Figure 6-15(b).

It is observed that even though no variability has been added to the eigenfrequencies or mode shapes, the identified damage pattern of Figure 6-15(a) presents some relatively small yet noticeable deviations from zero in some undamaged parts of the structure, which would correspond to false indications of stiffness change, such as in the regions of Pier 32 and the drop-in span. This problem arises from the combination of a highly localized actual damage pattern, as shown in Figure 6-15(b) for the present example, and a relatively coarse damage function.

Despite the problems, the results of Figure 6-15, still provide a reasonable and useful indication of where the damage might be located. In fact, based on these results, the damage element mesh can be refined to obtain a more accurate solution, i.e. a more precise location and a more accurate severity estimation of the damage in a second stage of damage detection.

Figure 6-16 shows an example of a refined piece-wise linear damage function mesh that may be used for a more accurate damage detection. In comparison to the original mesh of Figure 6-11, the mesh is refined in the area of suspected damage near the joint of the 
continuous drop-in span, while it has been made coarser in regions where the previous result shows there is no damage. The number of updating variables has been kept almost constant, only increasing from 22 to 23.

The pattern of stiffness correction factors $c_{e}$ identified by finite element updating using the refined damage function mesh is shown in Figure 6-17(a). As the figure shows, the damage is accurately identified from all 5 simulations of the FE updating algorithm. The results show that a properly refined damage function mesh can indeed lead to more accurate damage identification results, as long as the increased density of damage elements does not lead to an ill-conditioned problem.

\section{Scenario 3: Deterioration of piers}

Figure 6-18(a) shows the results of 5 simulations of the FE updating damage detection algorithm for the damage scenario of $10 \%$ stiffness reduction of the bridge piers. The results show that the method succeeds in identifying the piers as the main location where change of stiffness has occurred. However, from a quantitative point of view, the identified distribution of the damage is inaccurate, especially considering that this simulation does not yet include any variability in the modal parameters. A significant portion of the piers is falsely identified as having increased in stiffness. The reason is that the damage element mesh of the instrumented portion of the bridge includes only piers 31 and 32, while the damage occurs to all piers in the model (29 through 32). As a result, the method is forced to find a stiffness distribution within the domain of the given damage mesh that accounts not only for the changes occurring within the meshed region but also elsewhere. While it is possible in principle to extend to damage element mesh beyond the instrumented portion of the bridge, doing this would introduce more updating variables without a corresponding 
increasing in the measured information, which would lead to poorer conditioning of the optimization problem.

While the ideal case that the entire structure is instrumented may be unrealistic or that the damage occurrence is restricted to the instrumented/damage-element-meshed region of the structure, the structural deterioration scenario considered above $(10 \%$ uniform pier stiffness reduction) is modified to include stiffness degradation only in the two piers within the instrumented portion of the bridge to evaluate the effectiveness of the FE updating method for structural condition assessment here. As shown in Figure 6-18(b), in this case the damage is properly identified. The result is encouraging but it implies that the ability to properly detect distributed damage comes at the expense of having to instrument the entire structure.

\subsubsection{Damage detection results with variability}

\section{Scenario 1: Localized pier damage}

Figure 6-14(b) shows the results of 5 simulations of the FE updating damage detection algorithm for the damage scenario of $50 \%$ stiffness reduction in critical section 2 of pier 32. A weighting factor of $w_{s}=1$ is used for the mode shape residuals. The five simulations have different starting values for the updating variables while the realization of noise on the modal properties is kept constant. This represents the case in practice if only one set of measurements is available for the damaged and reference structures, i.e. there would be just one variability realization. As shown in Figure 6-14(b), the identified damage distribution indicates stiffness reduction in the damaged region, but also gives false indications of stiffness increases along the girder.

Figure 6-14(c) shows the results obtained after increasing the importance given to the relatively more accurate modal frequency residuals in the optimization problem by setting the 
weights of the mode shape residuals to a lower value of $w_{S}=1 / 10$. This leads to a slightly more accurate and stable solution. However, the result is still inaccurate at the drop-in span of the girder.

From a practical point of view, the accuracy of the results shown in Figure 6-14(c) would probably not be acceptable, potentially leading to erroneous conclusions about the state of the structure. One way to increase the accuracy in the identified damage patterns is by increasing the number of measurements. Namely, multiple independent samples of multiple measurements of modal properties can be collected for both the "undamaged" and "damaged" structures. The advantage is that the mean measured modal properties obtained from each sample would have different "realizations" of the measurement noise. This implies that the identified damage pattern obtained from each pair of "undamaged""damaged" samples would have a different error. Therefore, averaging the identified damage patterns would tend to reduce the error due to random noise, yielding a more accurate result. This is illustrated by the results of Figure 6-14(d). These results correspond to a second set of 5 simulations of the FE updating algorithm, in which, as opposed to the simulations of Figure 6-14(c), each simulation has a different realization of the random noise in the "measured" modal properties, as well as different random starting points for the updating variables $\theta$. This is meant to reflect what would occur if different independent samples of measured modal properties could be taken and used for damage detection, as discussed earlier. In practice, if 5 samples of size of $N=10$ were to be taken for both the undamaged \& damaged structure, the analysis of a total of 100 monitoring vibration datasets would be required. With each dataset consisting of 10 -minute time history records from 50 sensors at $100 \mathrm{~Hz}$ sampling rate, the total amount of data would be roughly $4.8 \mathrm{~GB}$. The results show that while each independent simulation may exhibit significant deviation from the actual 
damage pattern, the average identified damage pattern, shown in Figure 6-14(e), is reasonably close to the damage pattern identified without variability, more so than the single-sample result of Figure 6-14(c).

The results demonstrate the advantage of continuous long-term structural monitoring, which makes it possible to build a consistent long-term database with enough quantity of datasets to meet the challenges of damage detection of real complex civil engineering structures in the field. Nonetheless, given that there is still some uncertainty involved, in practice it is recommended to use the results only as an indicator of which region(s) of the structure should be checked in more detail, either through visual inspections, localized experimental methods (radiographs, acoustic, ultrasonic methods, etc.)

\section{Scenario 2: Damage at girder joint}

Figure 6-15(c) shows the identified patterns of element stiffness correction factors $c_{e}$ identified from 5 different simulations of the FE updating algorithm, taking the simulated variability in the measured modal properties into account. The results correspond to the damage scenario of $50 \%$ stiffness reduction at one of the joints of the continuous drop-in span, for which the actual damage pattern is shown in Figure 6-15(b). A weighting factor of $w_{S}=1 / 5$ is used for the mode shape residuals, which is selected after trying different values. As the figure shows, the FE updating method succeeds in indicating a stiffness reduction in the region of the actual damage, but at the same time, stiffness changes of comparable magnitude are falsely identified in regions of the structure that are actually intact.

Figure 6-15(d) shows the results that would be obtained if five independent samples of undamaged-damaged modal properties were taken, while Figure 6-15(e) gives the corresponding averaged result. Although there are some substantial errors remaining, the 
results of Figure 6-15(e) are significantly better than those of the single sample case (Figure $6-15(c))$

In practical application of damage detection of large complex civil engineering structures, the above results demonstrate that it is necessary to process and analyse large amounts of data, requiring significant computational effort and time. If a continuous monitoring system is available, the requirement of a large number of consistently acquired datasets can easily be met. Then the only other significant practical difficulty would be in regards to the system identification analysis of such a large amount of datasets. At present time, existing system identification methods tend to require significant input and judgement from the analyst in the system identification process, and automated system identification procedures may not be as reliable and give results of the same degree of accuracy.

As mentioned earlier, in theory it is possible to increase the accuracy of the damage identification results by using a more refined damage function mesh. The idea is that once a preliminary result, obtained using a "regular" damage function mesh, indicates the existence of damage in a specific region of the structure, a new refined damage function mesh with higher resolution of the damage function in the suspected damaged region can be designed. Unfortunately, a more refined mesh tends to lead to poorer conditioning of the optimization problem, which may result in higher sensitivity to noise disturbances. This problem is evaluated here by conducting the damage detection of damage scenario 2 with variability in the measured modal parameters using the refined mesh of Figure 6-16. The results are presented in Figure 6-17 (b) and (c). Despite the fact that the average result is acceptable, the figure shows that as compared to the case of the non-refined damage function, there is more instability of the identified damage pattern in the damaged region. The higher instability of the solution is attributable to the decrease in the quality of the Jacobian 
resulting from the mesh refinement, which makes it more difficult for the algorithm to distinguish the effect of changes in neighbouring elements on the value of the objective function.

\section{Scenario 3: Deterioration of piers}

Figures 6-20(c) and (d), show the results of the damage detection simulations corresponding to the damage scenario of uniform stiffness degradation of the piers (piers 31 and 32). This damage scenario represents the distributed degradation that may be expected as a result of long term degradation of material properties, in this case from long term exposure to sea water. In Figure 6-18(c), the results of 5 independent simulations of the FE updating algorithm are shown, corresponding to 5 independent samples (sample size $N=10$ ) being available for both the damaged and undamaged structures. Again, the variability in the measured mode shapes and frequencies of the undamaged and damaged structures significantly affects the damage identification results as compared to those obtained without variability presented in the previous section. The regions near the supports of the hinged drop-in span present higher variation of the identified damage pattern, which is consistent with the results obtained previously for the other damage scenarios. This suggests that the mode shapes and frequencies are not very sensitive to the change in stiffness in these regions. Nonetheless, the associated random errors tend to cancel out if a large enough quantity of independent data samples is used, i.e. the averaged damage detection results tend to converge to the right solution.

The results suggest that if enough consistently acquired monitoring datasets are available, it may be feasible to detect the kind of distributed stiffness degradation patterns expected from the gradual deterioration of the structural materials exposed to the operating environment with reasonable accuracy. The level of accuracy attained in the results of Figure 
6-18(d) may well be acceptable for practical engineering purposes, especially if the monitoring results are used as a preliminary diagnostic technique to be followed by more detailed localized inspections. The results indicate that the damage may be detected at relatively low stiffness reduction levels $(\% 10)$, and as such the damage detection results may give a useful indication of deterioration that can be used to take timely remedial measures.

\subsubsection{Conclusions}

One of the main challenges for the practical implementation and use of vibration monitoring data for structural health monitoring of civil engineering structures lies in the variability of the monitoring data arising from the different numerical and physical sources of uncertainty. The different uncertainties translate into significant variability in the identified structural features extracted from the data, which are used for damage detection, and tend to obscure the changes caused by damage. This chapter examines this challenge from the standpoint of the field-measured variability results obtained from long-term continuous monitoring data of the Confederation Bridge in Canada, and evaluates the implications for practical damage detection application in the field. A model of the field measured variability of the identified modal parameters is first developed and then used in damage detection simulations of the facility. The variability model accounts for the effects of measurement and environmental noises on the identified modal parameters of both the undamaged and damaged structures, as well as the amplitude-dependent variability of the mode shapes, which is a significant characteristic of the observed variability. The model is then used in damage detection simulations, to generate noise that is representative of actual monitoring data uncertainties, for evaluation of the validity, sensitivity and accuracy of a comparative detection algorithm by finite element updating. 
A recently introduced variant of the finite element updating method, using so-called "damage functions", which has previously shown promising performance for the Z-24 benchmark, is used as damage detection algorithm for the damage detection simulations presented here. Results from three localized and distributed damage scenarios considered, show that the variability in the data inevitably leads to some significant errors in the identified damage patterns, as expected. However, despite the significant variability in the modal parameters, especially in the mode shapes, it may still be possible to obtain some useful engineering information regarding the presence and location of damage from the output-only monitoring data using the FE updating approach. To counter the effects of random numerical and environmental noises, the use of multiple independent datasets is necessary, which requires the collection and analysis of relatively large amounts of monitoring data collected at different times and under different conditions for both the "undamaged" and "damaged" structures. This requirement can only be met through continuous long-term monitoring instrumentation. 


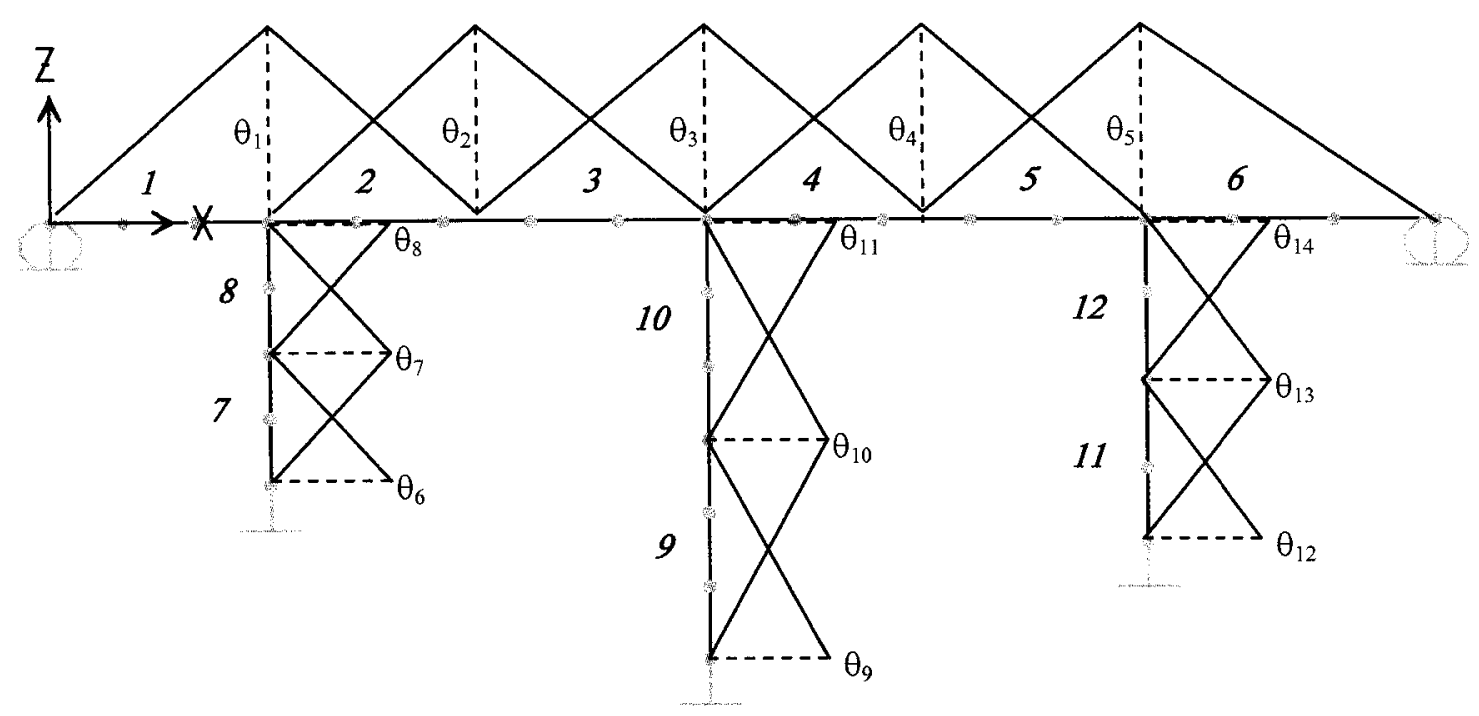

Figure 6-1: Damage elements (in italics) and piece-wise linear damage shape functions. 

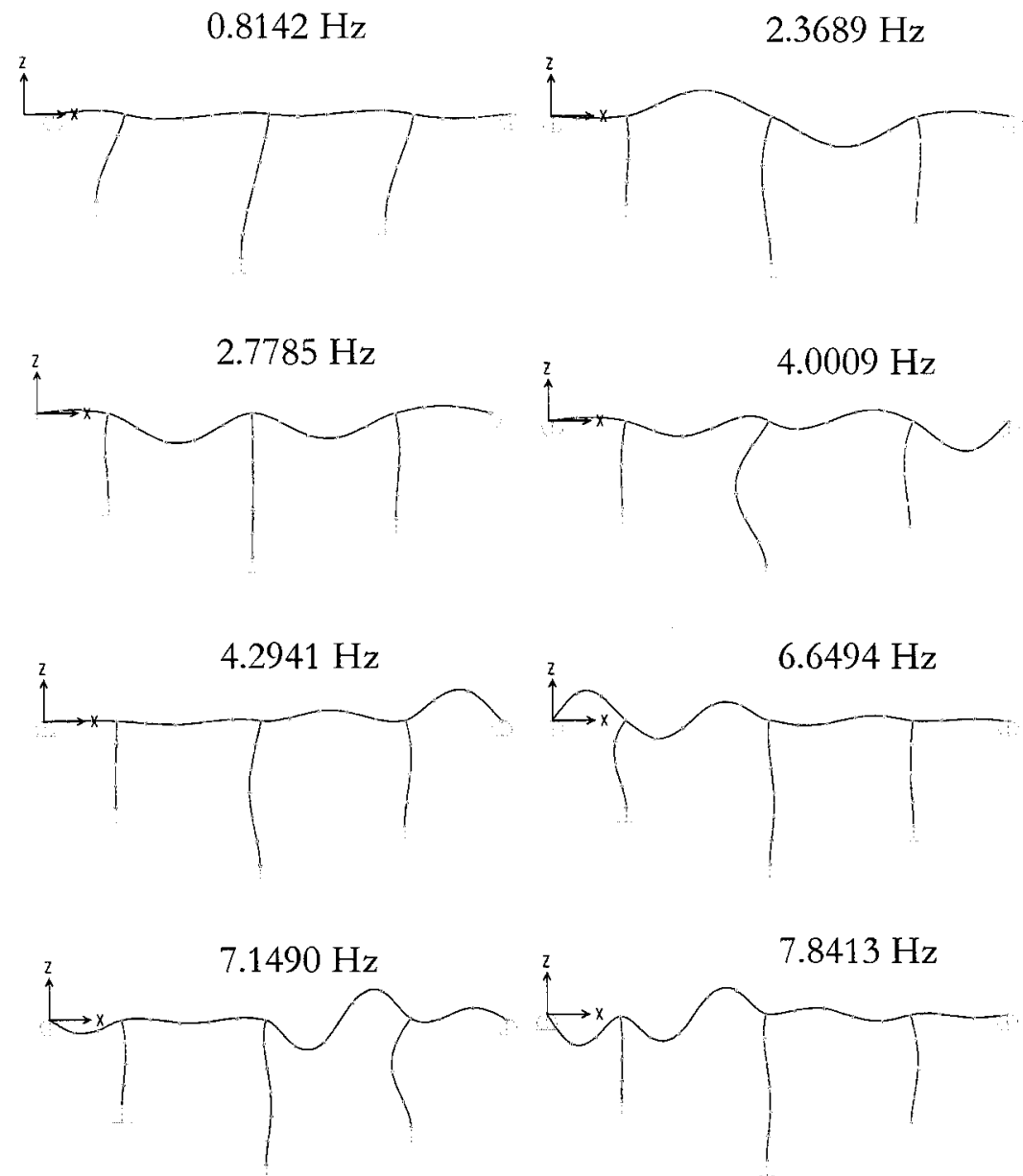

Figure 6-2: modes 1 to 8 used for damage detection. 

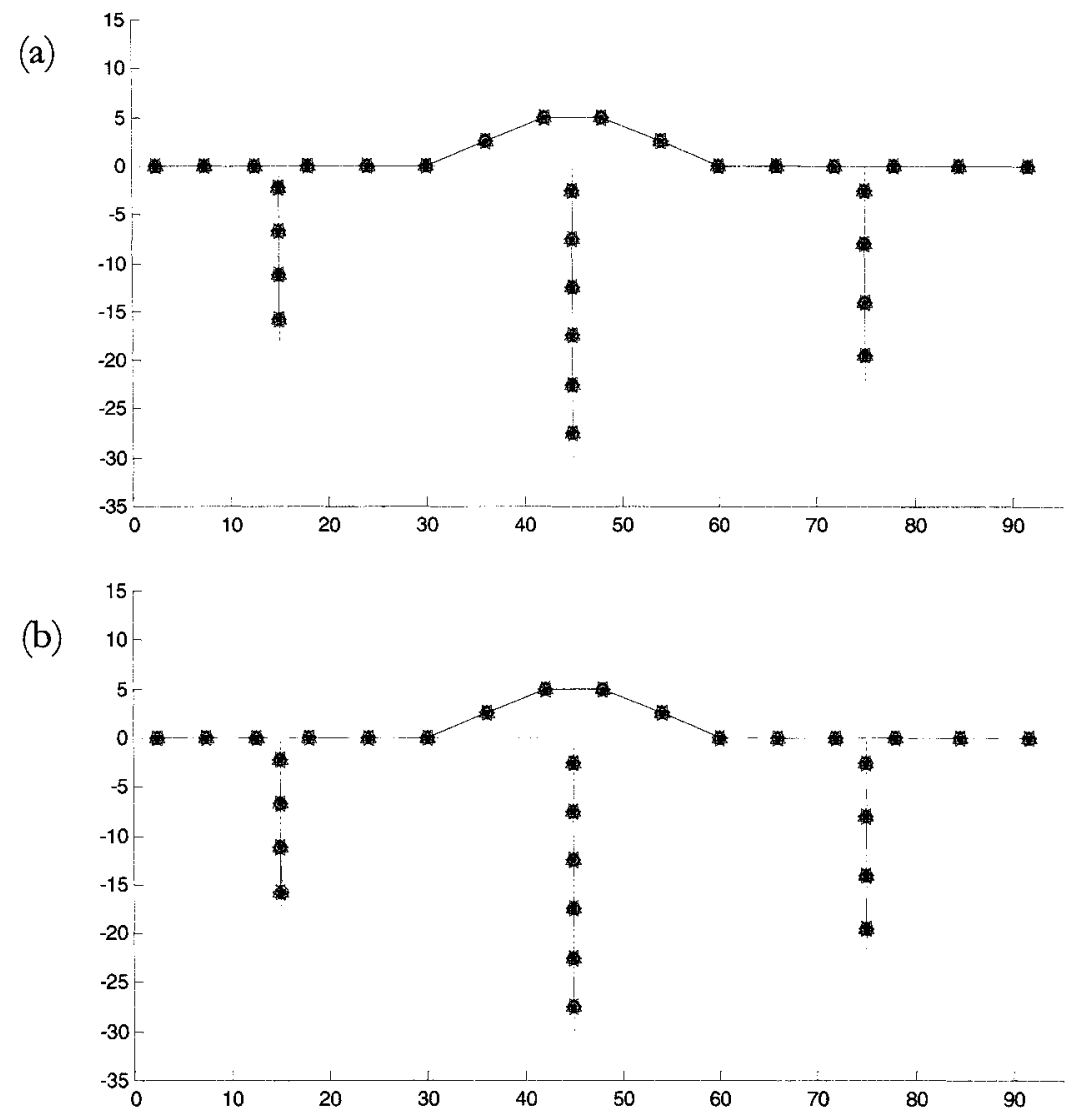

Figure 6-3: Distribution of element stiffness correction factors $c_{e}$ identified from 5 simulations of the FE updating algorithm with different starting points using (a) FE modes 1 to 8 as "measured modes"; (b) only FE modes $2,3,5,8$ as "measured modes". A scale of $1 \mathrm{~m}=0.1$ correction factor is used. Positive $c_{\mathrm{e}}$ 's plotted up $(\uparrow)$ for girders, to the right $(\rightarrow)$ for piers. Symbols simulations 1 to 5 : “-॰”, “-o",

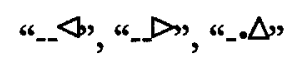



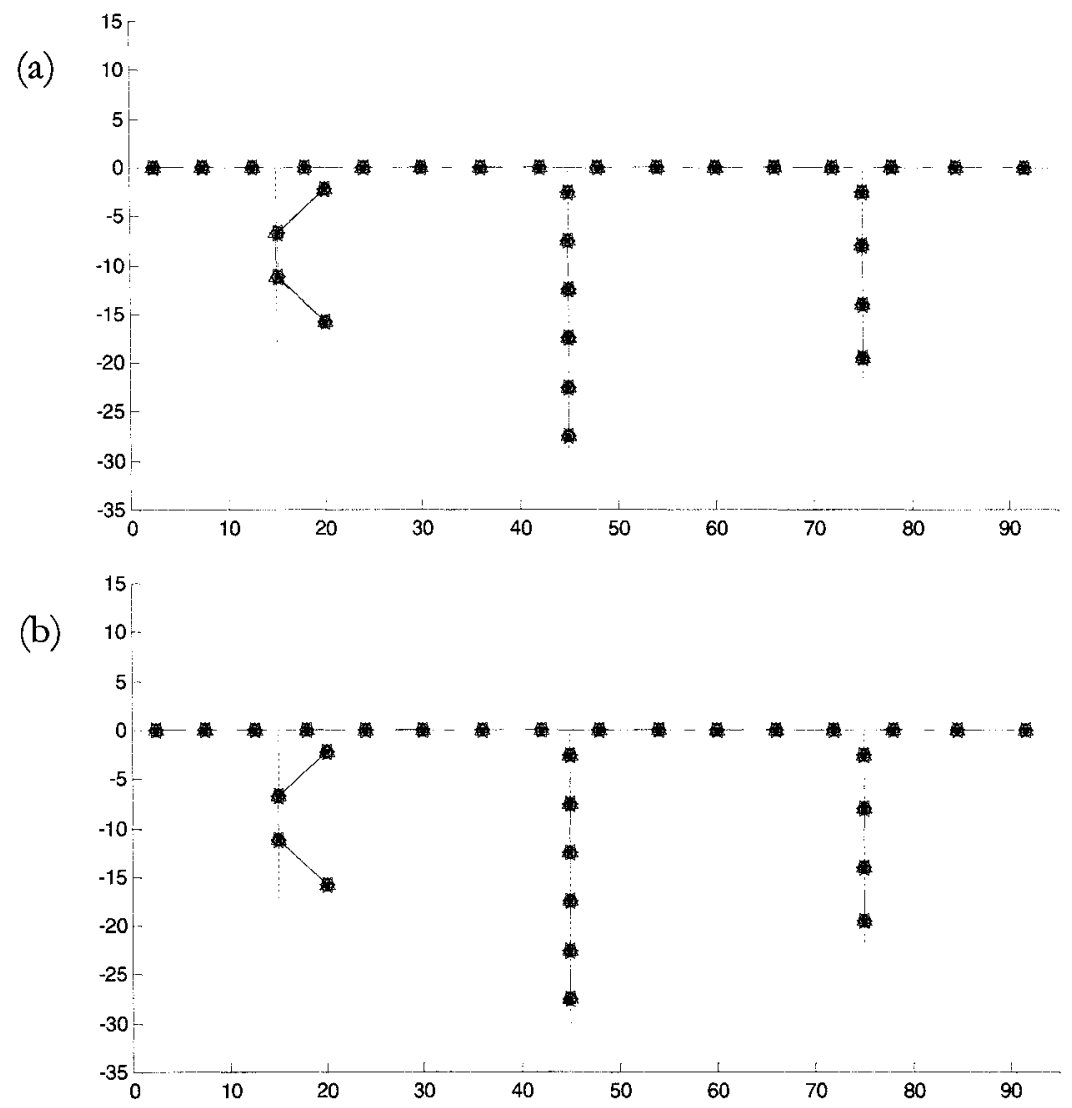

Figure 6-4: Distribution of element stiffness correction factors " $c_{e}$ " identified from 5 simulations of the FE updating algorithm with different starting points using (a)only FE modes 1 to 8 as "measured modes"; (b) only modes: $2,3,5,8 . \quad$ A scale of $1 \mathrm{~m}=0.1$ correction factor is used. Symbols for

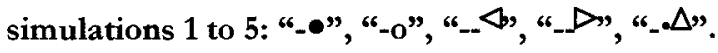



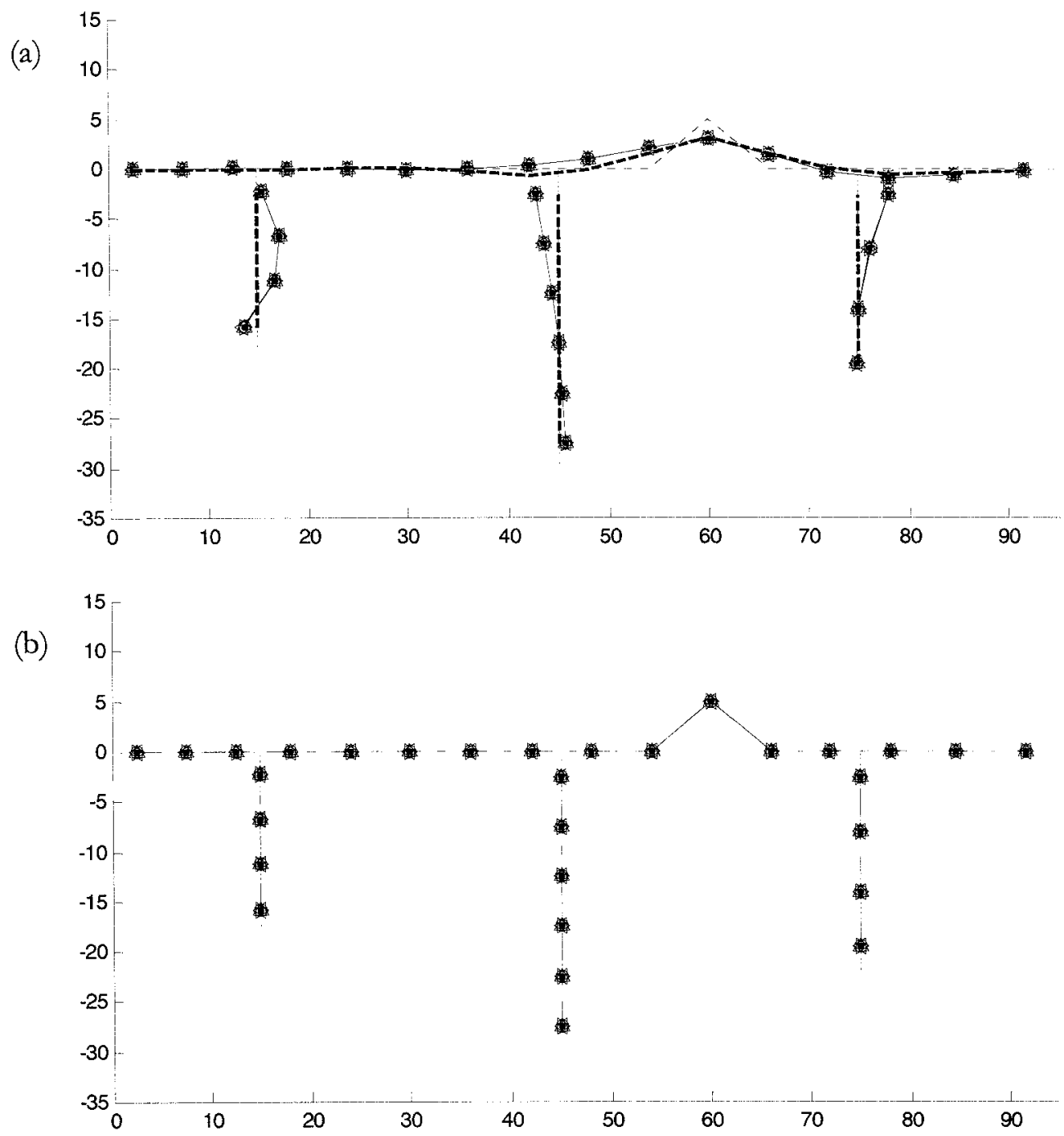

Figure 6-5: Element stiffness correction factors " $\mathrm{c}_{\mathrm{e}}$ " identified for damage scenario 3 from 5 simulations of the FE updating algorithm with different starting points, using modes 1 to 8 as "measured modes". (a) Results with regular damage mesh; actual damage pattern shown in dotted line; linear least squares fit to the actual damage shown in thicker dashed line; (b) results with refined

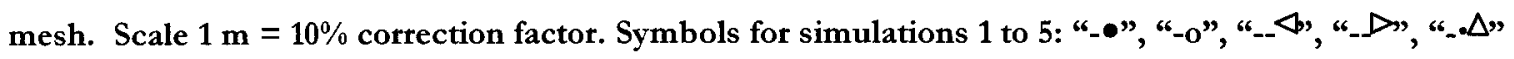
respectively. 


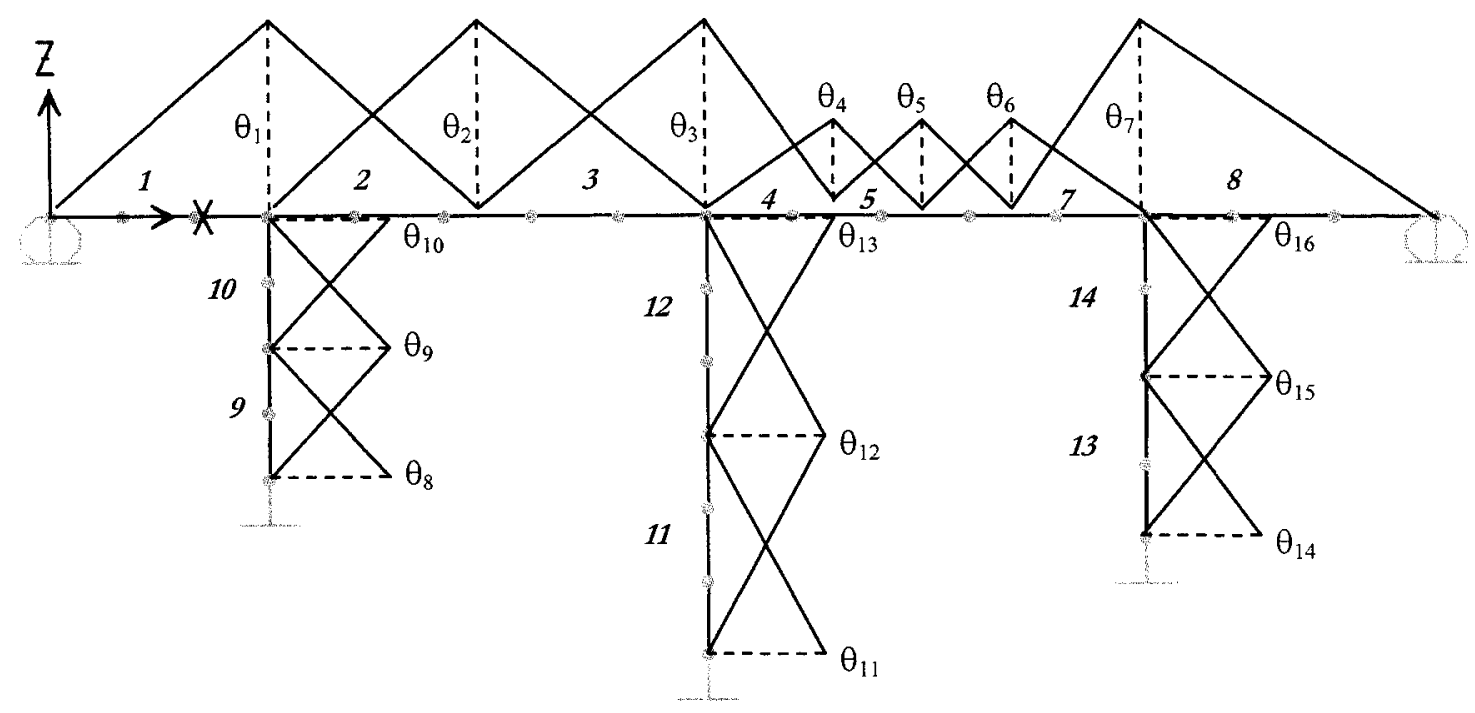

Figure 6-6: Refined damage element mesh and damage functions for damage scenario 3. 


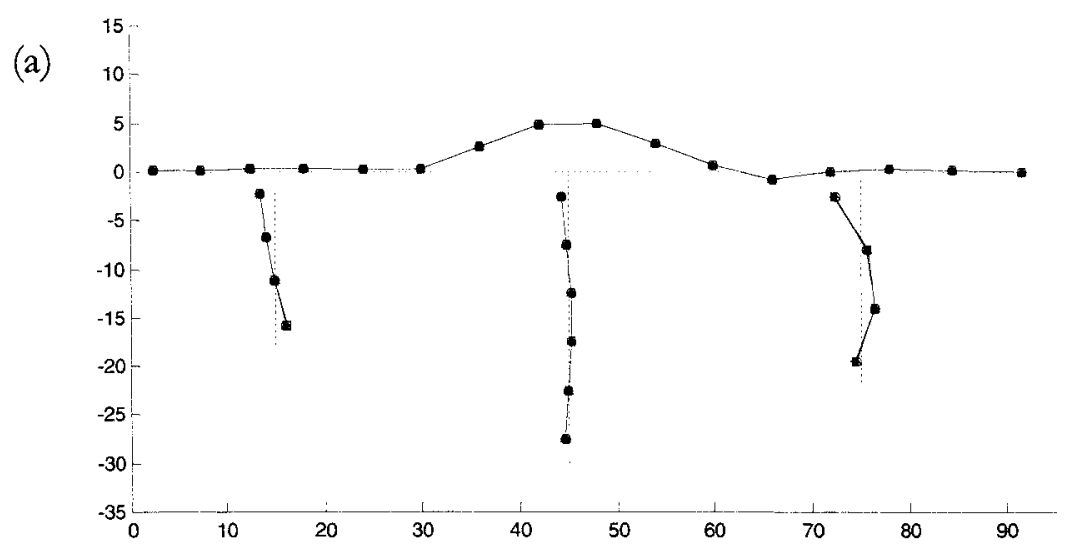

(b)

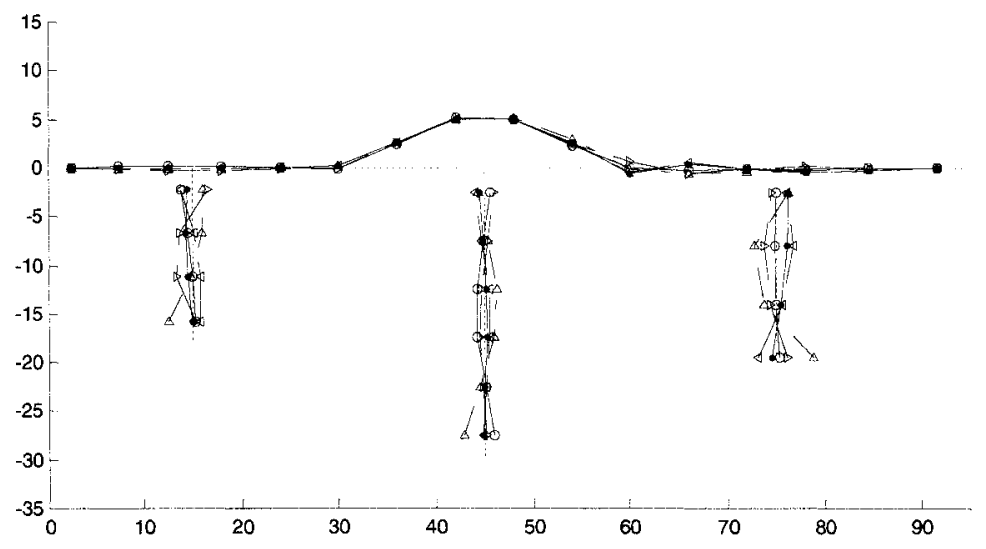

(c)

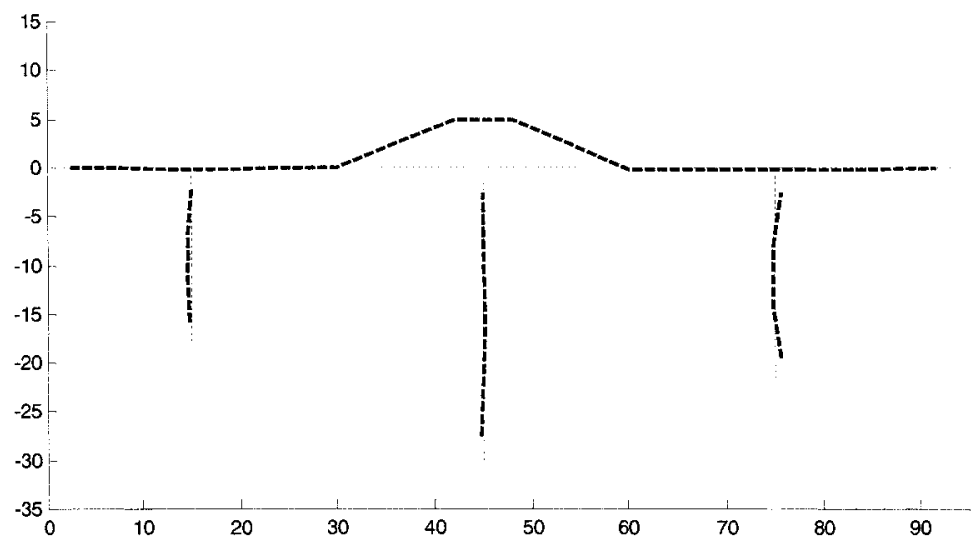

Figure 6-7: Distribution of element stiffness correction factors $c_{e}$ identified from 5 simulations of the FE updating algorithm using FE modes 1 to 8 as "measured modes" with different starting points and (a) fixed random noise in the measurements (Jacobian matrix condition number at the solution is 123); (b) different random noise in the measurements (condition number at solutions varies between 114 and 123). (c) Average of “(b)". A scale of $1 \mathrm{~m}=0.1$ correction factor is used. Symbols for simulations 1

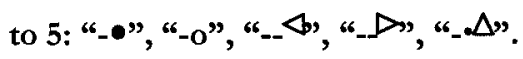




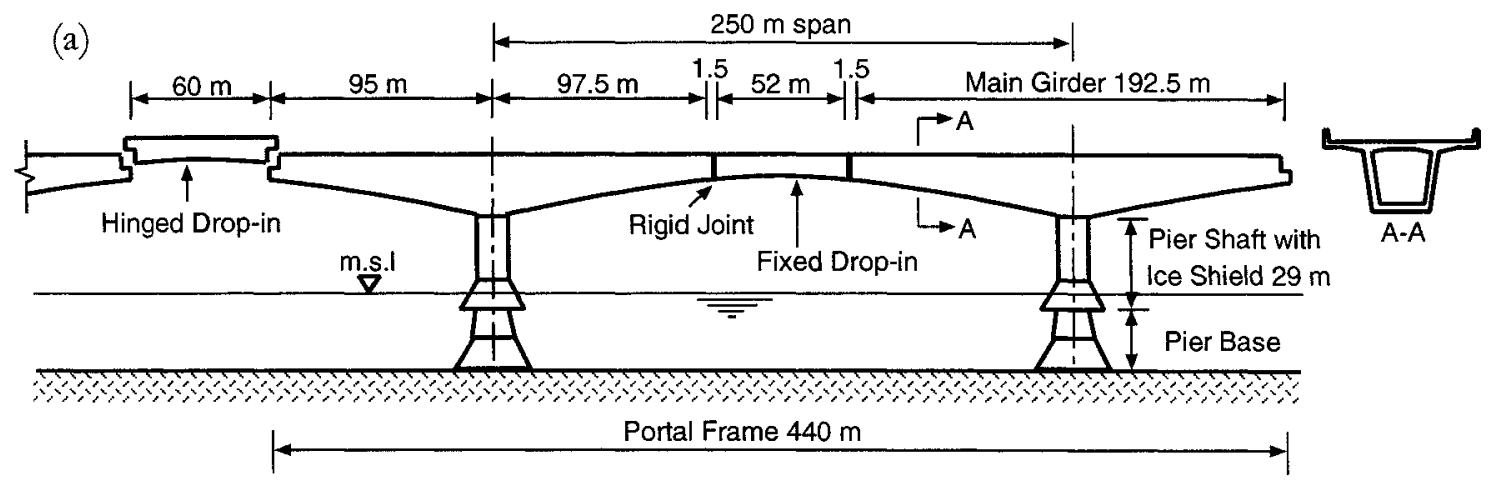

(b) 7

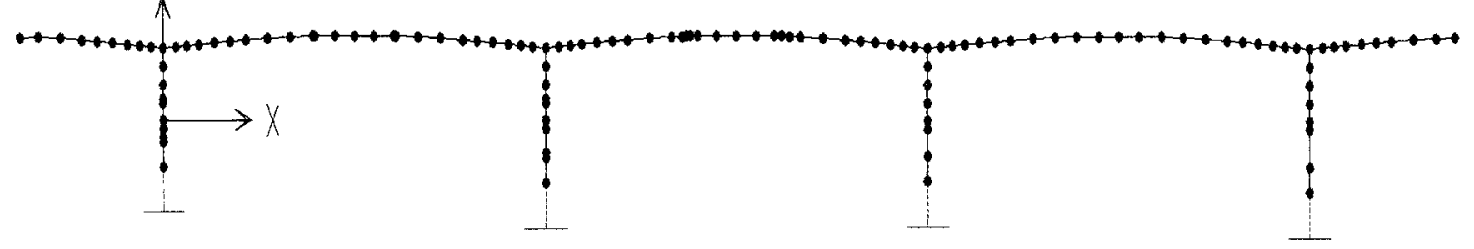

Figure 6-8: (a) Dimensions and main components of typical structural module; (b) beam element FE model of Confederation Bridge. 


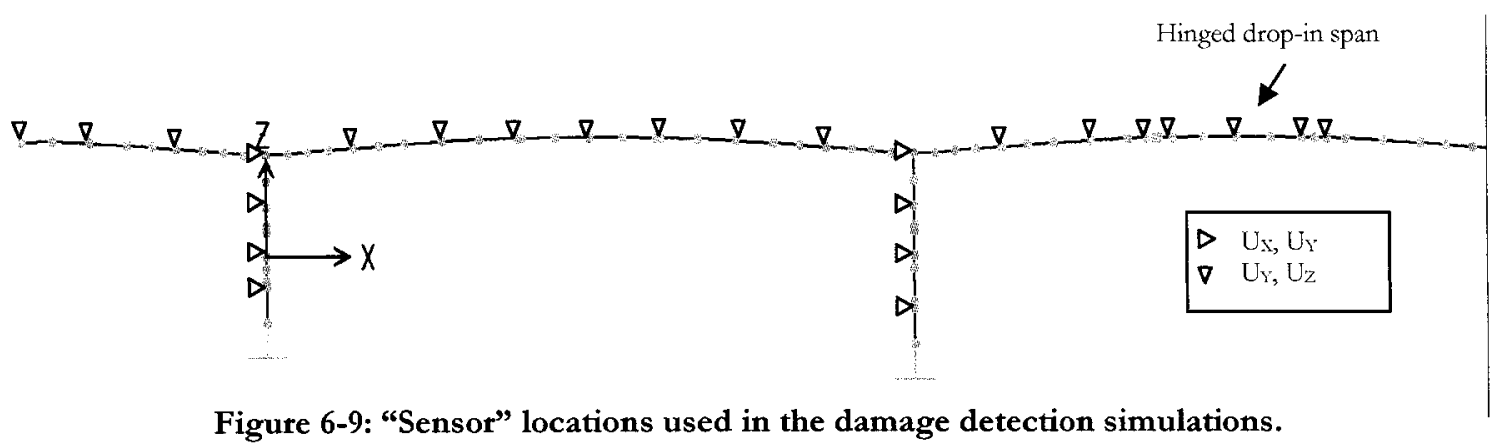

Figure 6-9: "Sensor" locations used in the damage detection simulations. 


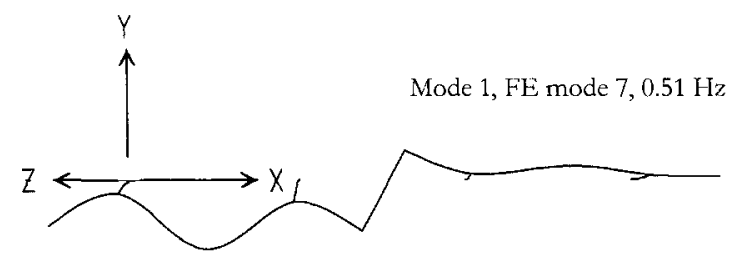

Mode 3, FE mode 10, $0.69 \mathrm{~Hz}$

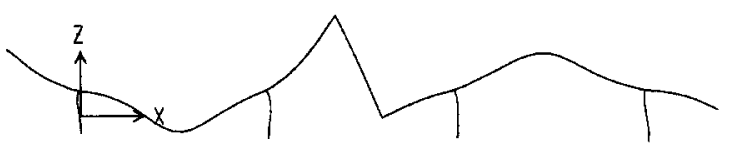

Mode 5, FE mode $13,0.91 \mathrm{~Hz}$

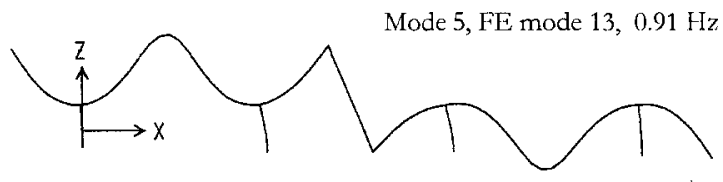

Mode 7, FE mode $25,2.60 \mathrm{~Hz}$

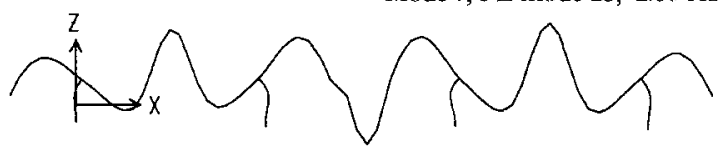

Mode 9, FE mode $27,3.07 \mathrm{~Hz}$

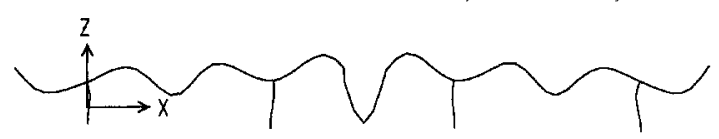

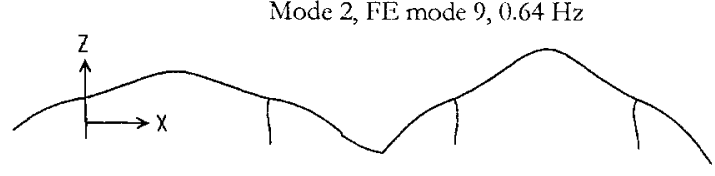

Mode 4, FE mode $12,0.88 \mathrm{~Hz}$
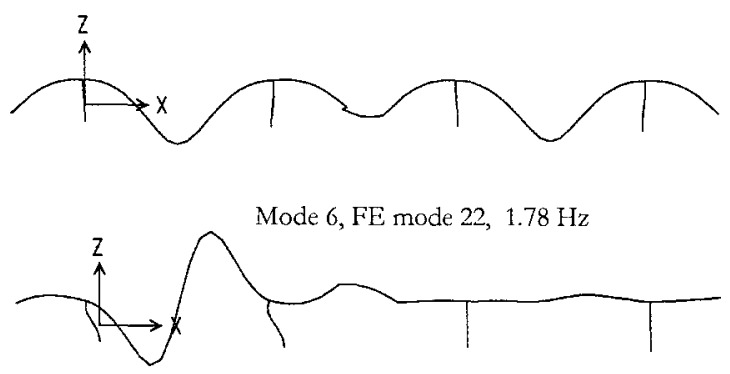

Mode 8, FE mode 26, $2.73 \mathrm{~Hz}$

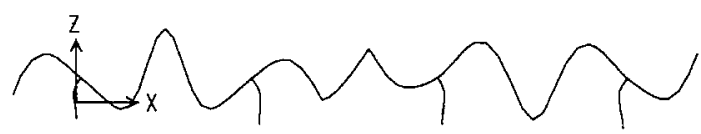

Mode 10, FE mode $40,5.0 \mathrm{~Hz}$

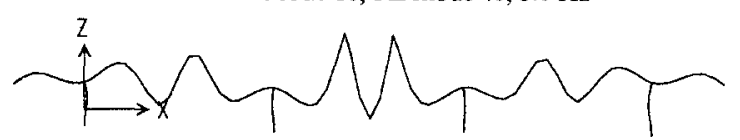

Figure 6-10: Modes of Confederation Bridge used as "measured" modes for damage detection. 


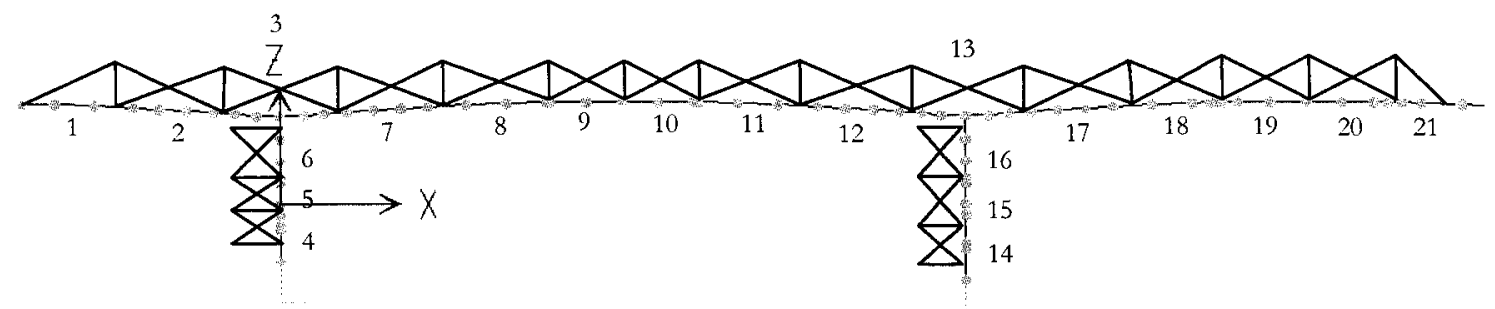

Figure 6-11: Damage element mesh and local damage functions for Confederation Bridge 


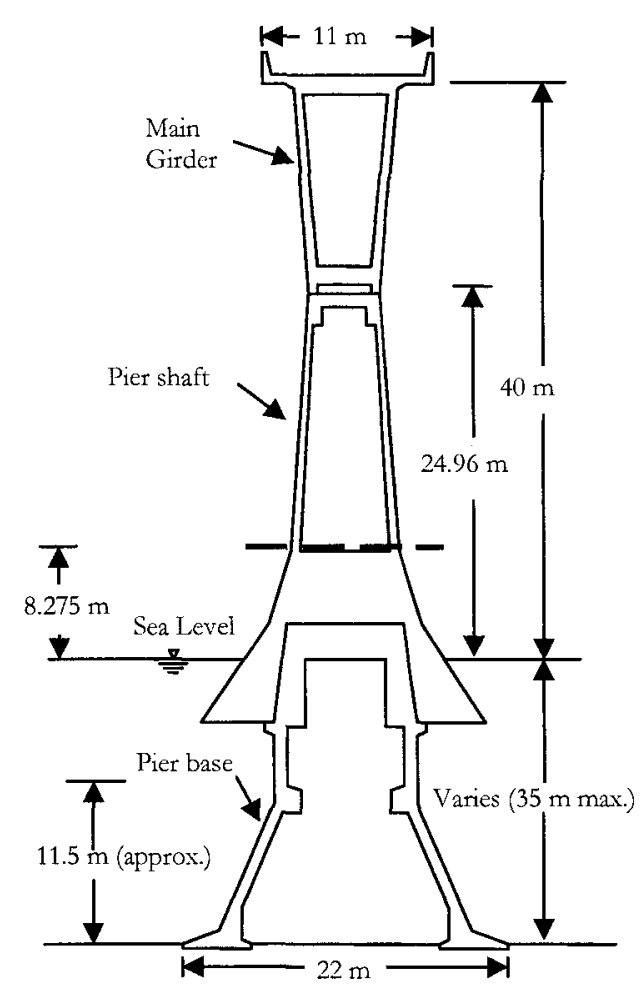

Figure 6-12: Bridge pier cross section showing location of simulated damage considered in damage scenario 1 , indicated by dotted line. 


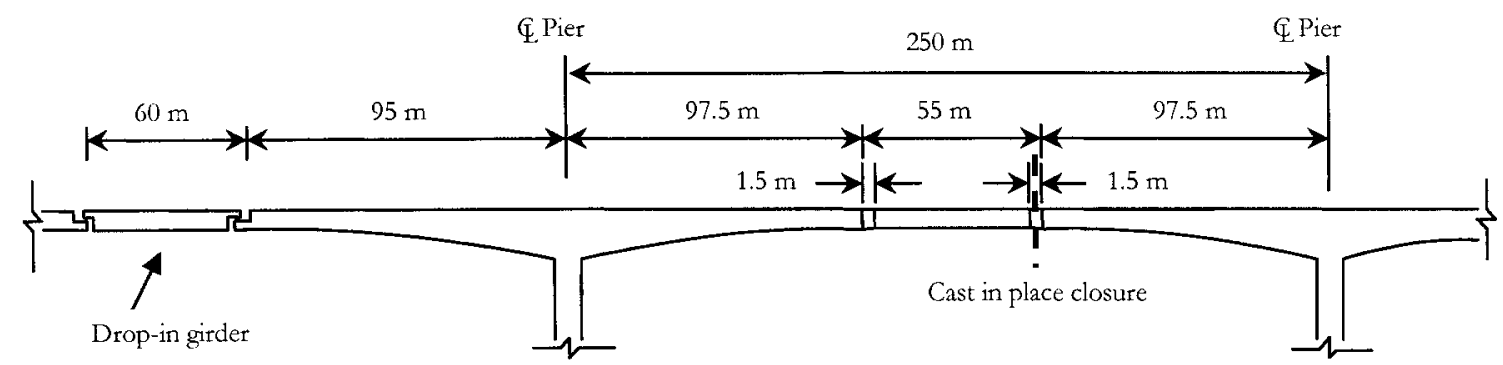

Figure 6-13: Bridge profile showing the location of simulated damage in girder joint considered in damage scenario 2 , indicated by dotted line. 

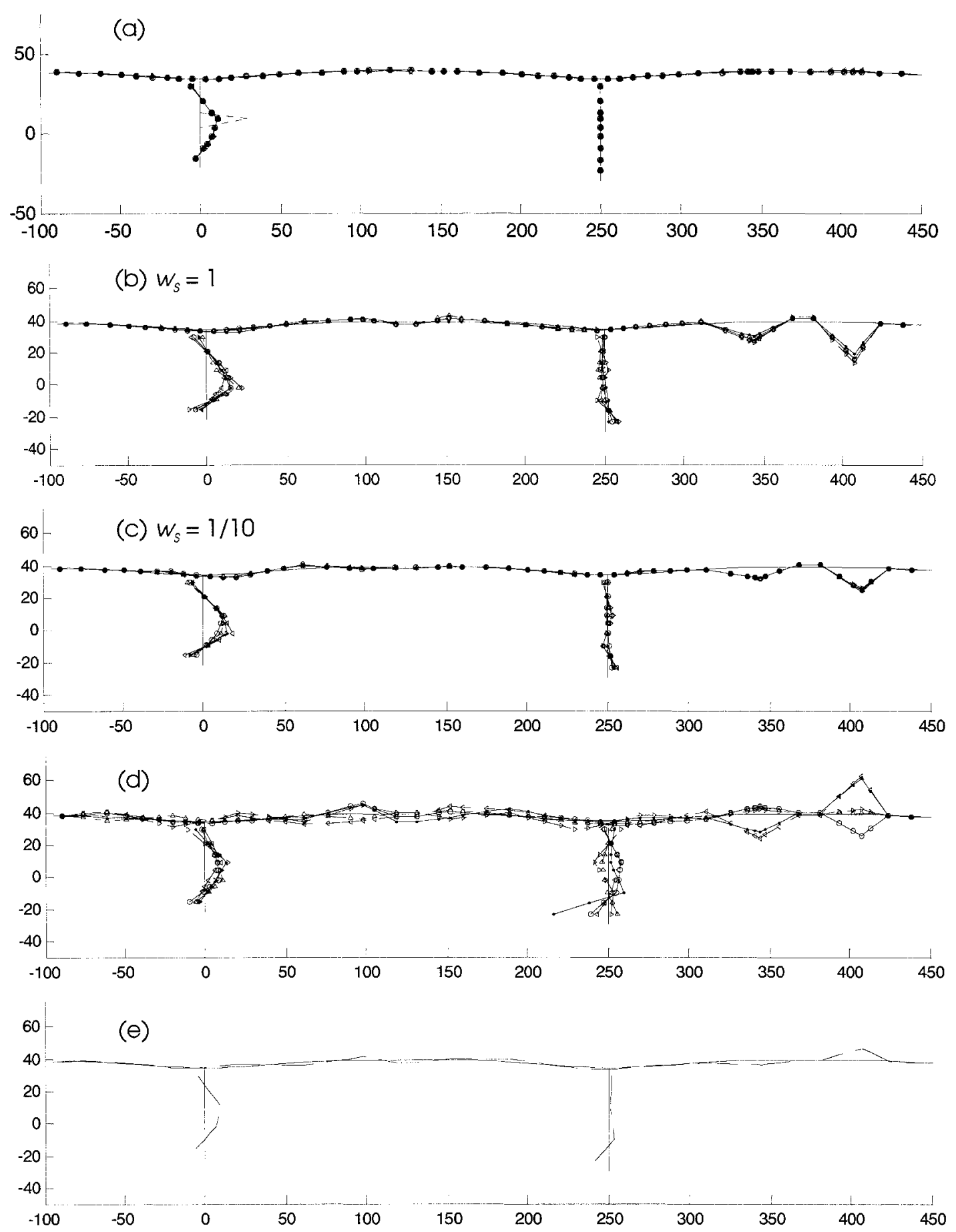

Figure 6-14: Identified pattern of element stiffness correction factors $c_{\mathrm{e}}$ identified from 5 simulations of the FE updating algorithm with different starting points for damage scenario 1; (a) without noise in the measured modal properties; (b) and (c) with noise; (d) with different noise realizations; (e) average

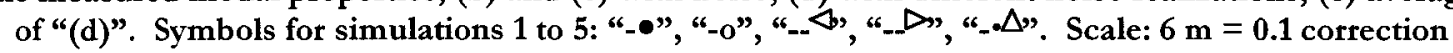
factor. Positive $c_{e}$ plotted up $(\uparrow)$ for girders, to the right $(\rightarrow)$ for piers. The actual damage pattern is shown in dotted line. 

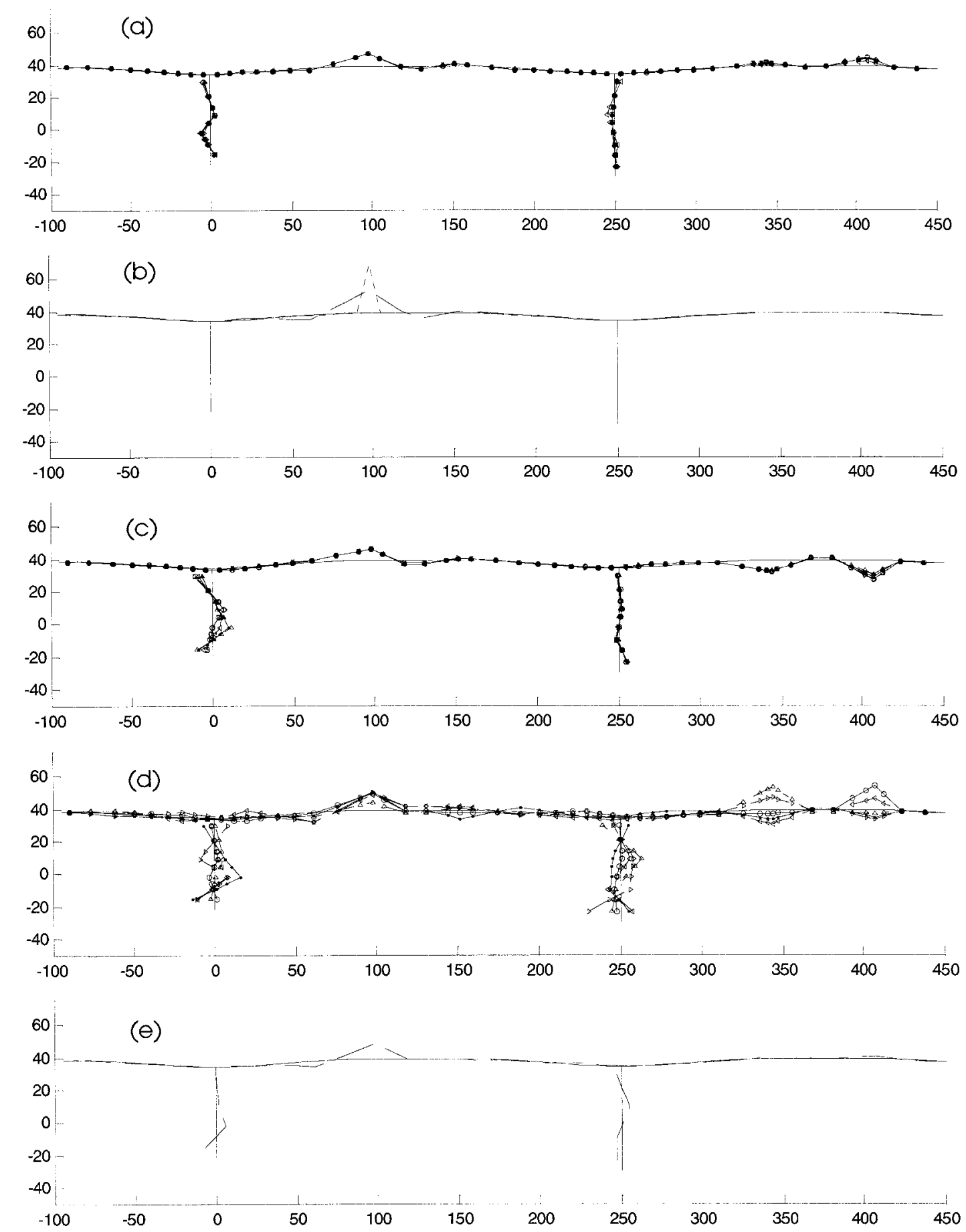

Figure 6-15: Identified pattern of element stiffness correction factors $c_{e}$ identified from 5 simulations of the $\mathrm{FE}$ updating algorithm with different starting points for damage scenario 2 ; (a) without noise in the measured modal properties; (b) actual damage pattern (dotted), best fit of damage function to the actual damage (dashed); (c) with noise; (d) with different noise realizations; (e) average of "(d)".

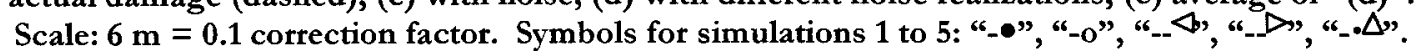




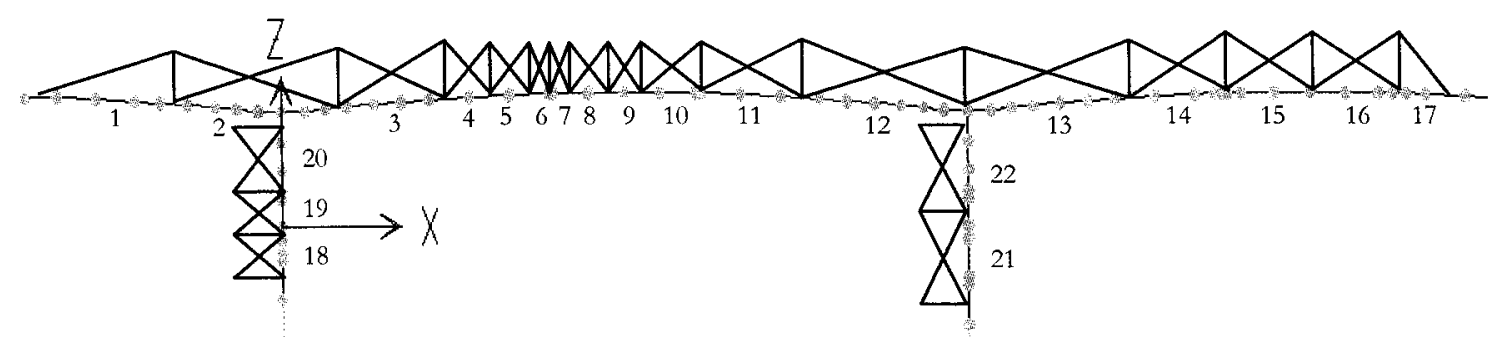

Figure 6-16: Refined mesh for damage scenario 2, based on preliminary damage detection. 

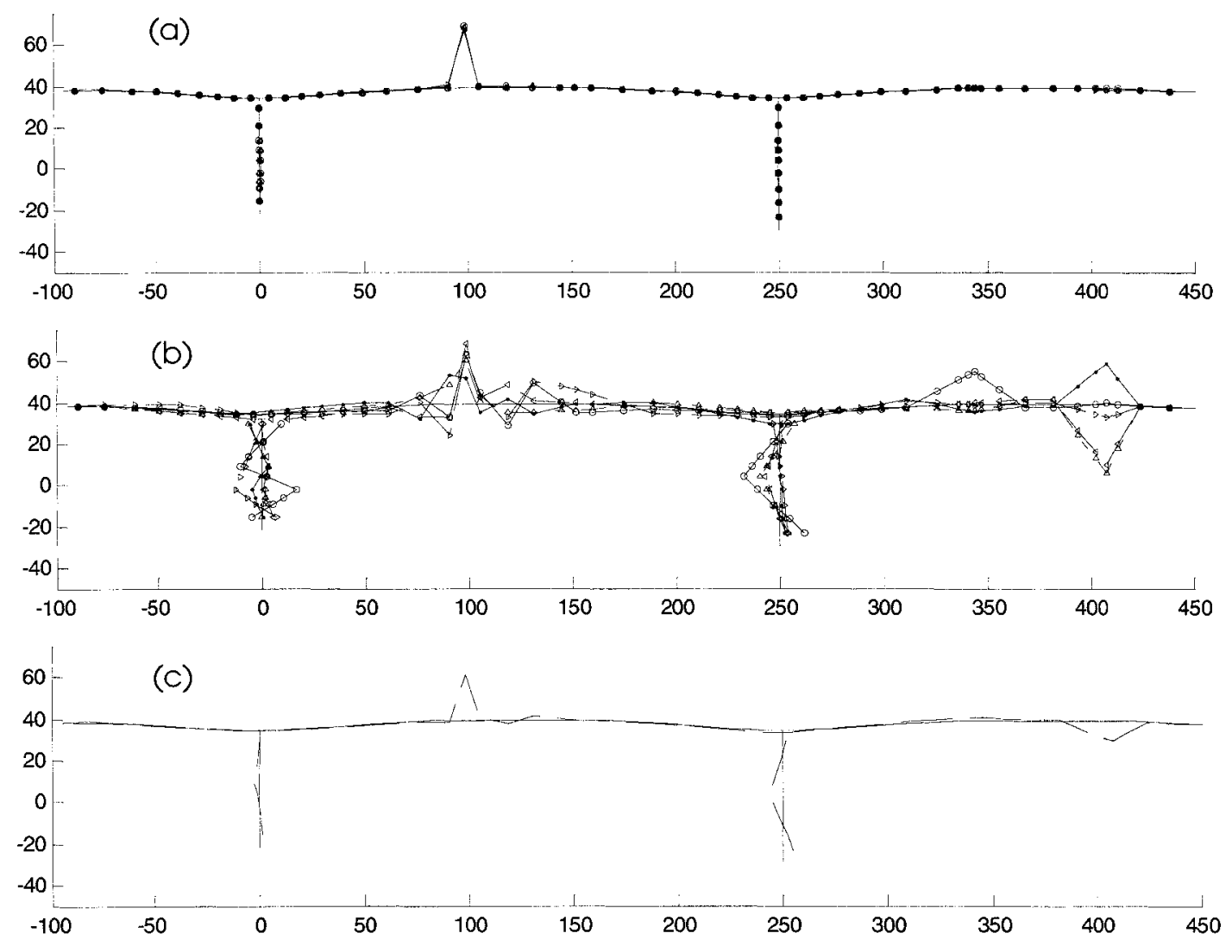

Figure 6-17: Identified pattern of element stiffness correction factors $c_{e}$ identified from 5 simulations of the FE updating algorithm with different starting points for damage scenario 2 using refined mesh; (a) without noise in the measured modal properties; (b) with different noise realizations; (c) average of the results of “(b)". Scale: $6 \mathrm{~m}=0.1$ correction factor. Symbols for simulations 1 to 10 : “_॰”, “-o", “_ «", “-

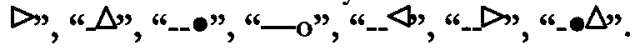


(a)
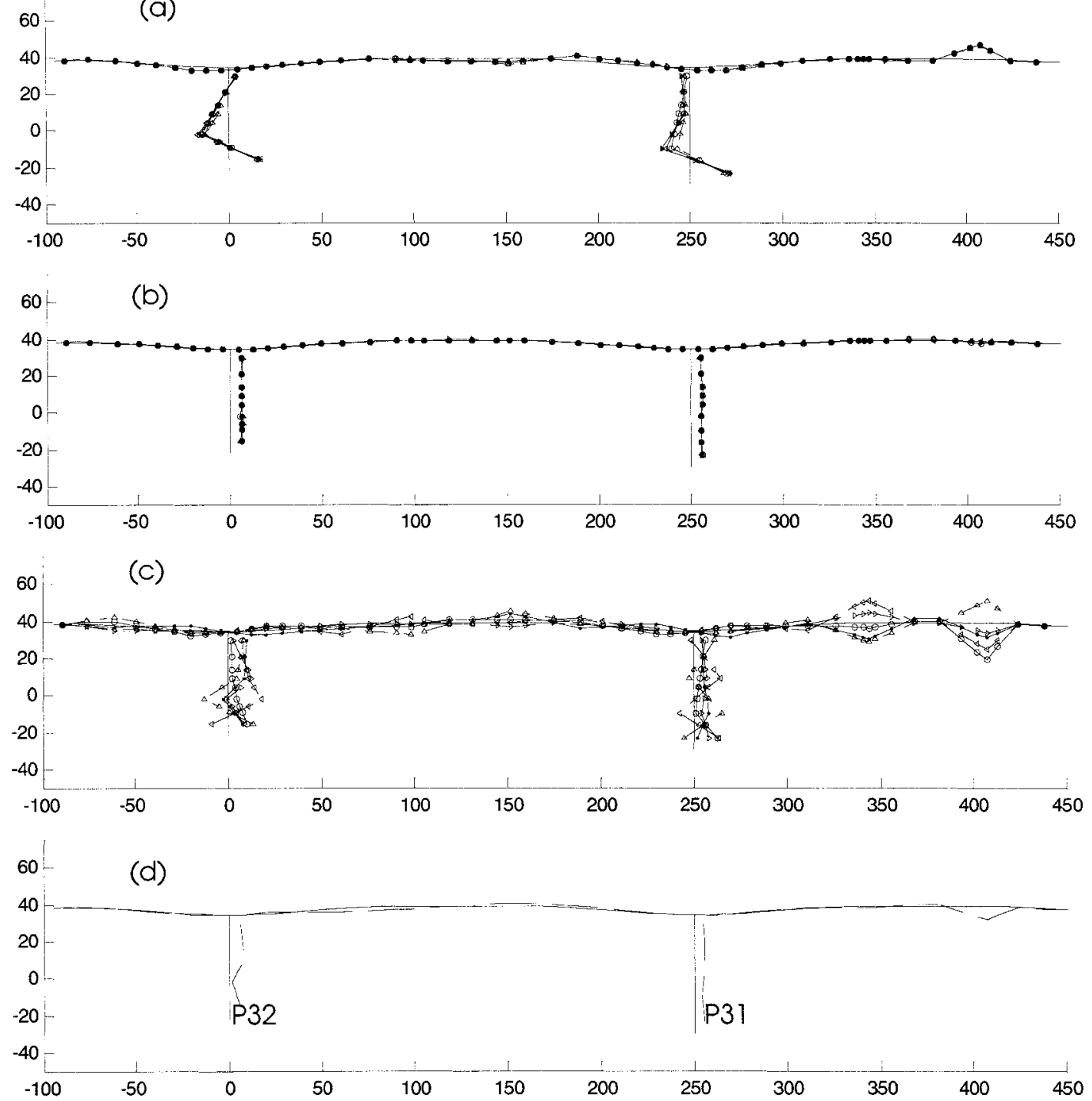

Figure 6-18: Identified pattern of element stiffness correction factors $c_{e}$ identified from 5 simulations of the FE updating algorithm with different starting points for damage scenario 3. (a) Damage of all piers, without noise; (b) damage of piers within instrumented segment, without noise; (c) with different noise realizations; (d) average of "(c)". Scale: $6 \mathrm{~m}=0.1$ correction factor. Symbols for

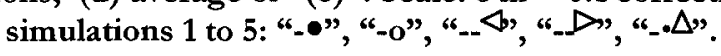




\section{Chapter 7. Conclusions and recommendations}

\subsection{General remarks}

Some brief final remarks are presented in this section to give the reader a global perspective of the work presented in this thesis as well as suggesting possible routes for continued research on the subject. For conclusions regarding specific topics the reader is referred to the conclusion section of the corresponding chapters.

Current practices of structural condition assessment are based mainly on routine visual inspections or condition surveys. This approach has important limitations such as the inability to provide quantitative information about how the local deteriorations, damages, or retrofits and repairs have affected the global structural integrity and the fact that the site visits can only be conducted at relatively infrequent periodic intervals, among others. Over the last decades, developments in sensing and information technology have made it possible to monitor various structural responses and data regarding the operations of the structures with a high level of detail and accuracy. The data may include vibration responses, short and long-term deformations, temperature profiles, strains, and wind and weather data, to name a few. The availability of such information has opened up whole new avenues for investigation. Considerable research efforts have been directed towards finding how to use the collected response data for structural condition assessment. One particular kind of data has received particular attention, and that is the vibration response data. The reason for its attractiveness is that this data is relatively cheap to collect; it is non-intrusive, it implies no service interruptions and it is suitable for the continuous monitoring of the structures. Also, the vibration responses carry the essential information about the structural health: changes in the 
vibration frequencies reflect changes in stiffness and changes in the vibration mode shapes reflect changes in the distribution of the stiffness.

Part of the motivation for research in this area comes from the potential of the monitoring data to be used for on-line structural monitoring and condition assessment, which would provide timely information about the structural integrity and serviceability to operators and owners of the infrastructures immediately after the occurrence of events such as earthquakes, collisions, blasts, etc. However, so far the task of obtaining reliable, accurate and useful engineering information from the monitoring data even in an "off-line" mode has proven to be quite challenging.

One of the main difficulties stems from the uncertainties and errors in the monitoring data. The sources, magnitude and implications of these uncertainties have been extensively discussed in this thesis. Essentially, the implication of the uncertainties is that they tend to obscure the typically small changes in the structural parameters produced by damage or deterioration. Of the main goals of this thesis was to determine just how significant this masking effect is expected to be for real operational structures under varying environmental and loading conditions and to determine whether the difficulties seem to be surmountable or not. The results obtained in this research are promising in this respect. In particular, the methodology of finite element updating shows good potential for vibration based structural condition assessment. The findings of this study indicate that when this technique is used in conjunction with information from continuous monitoring data, which offers the possibility of using multiple independent datasets - collected at different times under different conditions -, a good portion of the random variability otherwise present in the damage detection results can be effectively cancelled out. Furthermore, continued improvements in the technology and manufacture of sensing and data acquisition hardware 
and software systems are likely to result in recorded data that is cleaner of measurement noises. Also, developments of new types of sensors, like fibre optic sensors, could make it feasible to increase the spatial resolution of the instrumentation grid. Such developments would enhance the accuracy of condition assessment results.

\subsection{Summary of contributions}

The main contribution of this thesis to the field of structural health monitoring is that this work presents the first systematic and comprehensive evaluation of the variability of real world long-term continuous vibration monitoring data from a large scale operational civil engineering structure, and examines its impact on the feasibility of applying structural health monitoring technology to structures in the field. This work includes:

- The identification and separate quantification of noise contributions from both numerical and physical sources of error and uncertainty.

- The determination of the role of temperature on the variation of the structural eigenfrequencies

- The identification of significant amplitude dependent behaviour in the variability of the mode shapes.

- The development of a model to generate noise in simulated measured values of mode shapes and eigenfrequencies, in accordance with the behaviour observed from the data, to be used in any comparative damage detection simulation based on mode shape and frequencies.

Other contributions can be summarized as follows:

- The verification of design assumptions and dynamic behaviour of the Confederation Bridge, by comparison of the expected dynamic properties with 
the values extracted from the monitoring data. Overall, the dynamic behaviour is found to be in good accord with what should be expected from the design drawings and specifications. Assumptions of elastic material properties and damping ratios have been found to be reasonable.

- The concept and development of the modular software application as a prototype of the kind of platform that could be used for the structural monitoring of civil infrastructure components and networks on a real-time basis.

\subsection{Recommendations for future research}

For future work, the next logical step is to test the damage detection scheme presented in this thesis using long-term continuous monitoring data of a structure in operation. An alternative is to set up a permanent monitoring system on a typical highway bridge. Since this is one of the most common types of civil structure, the impacts of any improvements in the condition assessment technology of this class of bridges would probably be the most economically significant. Using the permanent monitoring system, baseline information for calibration of the reference FE model of the "undamaged" structure could be gathered over a short, initial period of time (of the order of a few weeks). Then, after a period of a few months, ideally in a different season of the year, controlled damage scenarios could be introduced in the structure, provided that they do not compromise the structure's safety and serviceability. Such a study would provide ample reassurance of whether or not the damage induced changes in the structural responses can be reliably detected at reasonably early stages under the inevitable environmental changes and measurement noises.

Further investigations should be carried out into the problems of data nonstationarity and non-whiteness of the stochastic input terms - i.e. the exciting forces - and 
their effect in the system identification process. The investigations could examine whether new data analysis and system identification techniques proposed recently for non-stationary data, such as the Hilbert Huang Transform (HHT) (Huang et al. 1998, Yang et al.2003), would lead or not to more accurate system identification and to reduced errors in the damage detection. Furthermore, it may be possible to take advantage of the time-frequency descriptions of the data given by the non-stationary data analysis techniques to track the behaviour of the data in time and to detect the occurrence of changes in the data associated to damage. Some work has already been conducted in this direction (Yang et al. 2004). In regards to the issue of non-whiteness, it is possible that some knowledge of the spectral pattern of the inputs may be obtained (e.g. for the wind loading, as well as traffic and ice loadings) either from field data or previous studies. It should be investigated whether this knowledge may be incorporated into the system identification, as suggested by Bogunović Jakobsen J. (1995), and be used to obtain increased resolution and accuracy in the identification and condition assessment of the system.

Another issue in vibration based condition assessment that requires attention, especially in the case of on-line condition assessment, is the development of algorithms to detect sensor failures. Just as the structures they are installed on are subjected to attack from various environmental agents, so are on-site the sensing and data acquisition equipment. These are exposed to marine salts, voltage peaks from lighting strikes, temperature and humidity extremes, etc. The signals from failed or problematic sensors usually exhibit distinct characteristics, such as near zero amplitude, sudden peaks, etc., which can potentially be used as the basis to detect and discard them. The issue of sensor "health" monitoring should not be overlooked given that the inclusion of corrupted data for condition assessment would probably lead to completely erroneous results. Once this is done, alerts regarding sensor 
malfunctions can be issued - perhaps as part of periodic system status reports - so that the appropriate repairs and replacements can be done in a timely manner

Part of the work of this thesis was devoted to the development of computer tools to facilitate the interpretation of the monitoring data. Software of this kind is an essential component of any monitoring system. Without it the monitoring data would have little or no meaning. The overall concept as well as individual features and functions of the modular application developed in this work are a prototype for the kind of software platform that can be used as a monitoring tool in practice. As recommended future work, the application can be re-developed as a stand-alone web-based application, in a language such as Java, which would make the application more versatile and accessible to the parties involved. Also, the application can be expanded and adapted for data management and on-line monitoring and assessment of multiple monitored structures, such as would be necessary for the centralized monitoring of highway bridge networks. 


\section{References}

1. Abe M., Fujino Y., Yanagihara M. and Sato M. (2000). "Monitoring of Hakucho Suspension Bridge by ambient vibration measurement", Proceedings of SPIE - The International Society for Optical Engineering, Nondestructive Evaluation of Highways, Utilities, and Pipelines IV; Vol. 3995, pp. 237-244. DOI: 10.1117/12.387815

2. Abe M, Siringoringo DM. (2003). Structural health monitoring of long span bridges. Proceedings of the First International Conference on Structural Health Monitoring and Intelligent Infrastructure, 13-15 November 2003, Tokyo, Japan, Vol. 1, pp. 171-179.

3. Aitcin, P.-C. (2002). Built to last: the Confederation Bridge, Canada. Concrete, 36(1):3840.

4. Alampalli, S. (1998). Influence of in-service environment on modal parameters. Proceedings of the $16^{\text {th }}$ International Modal Analysis Conference (in Proceeding of SPIE, Vol. 3243), pp. 111-116.

5. Anglin C.D., Nairn R.B., Cornett A., Dunaszegi L., and Turnham J. (1997). Bridge pier scour assessment for the Confederation Bridge, Canadian Journal of Civil Engineering, 24: 934-940

6. Avitabile P., O’Callahan JC, Milani J. (1989). "Comparison of system characteristics using various model reduction techniques." Seventh International Modal Analysis Conference, Las Vegas, Nevada, February 1989.

7. Benveniste A. and Fuchs J.-J. (1985). Single sample modal identification of a nonstationary stochastic process. IEEE Transactions on Automatic Control; AC-30(1): 66-74.

8. Bernal D. (2002). "Load vectors for damage localization", Journal of Engineering Mechanics, 128(1):7-14.

9. Bernal D. (2003) "A flexibility based approach for the characterization of damage Application to phase IIe of the IASC-ASCE benchmark study." Proceedings of IMACXXI: A Conference on Structural Dynamics, February 3 - 6, 2003, Kissimmee, Florida.

10. Bernal D. (2006) "Flexibility based damage localization from stochastic realization results", Journal of Engineering Mechanics, 132(6):651-658. 
11. Bogunović Jakobsen J. (1995). Fluctuating Wind Load and Response of a Line-like Engineering Structure with Emphasis on Motion-Induced Wind Forces, Ph.D. Thesis, Department of Structural Engineering, The Norwegian Institute of Technology, University of Trondheim, Norway.

12. Brincker R. and Andersen P. (2003). "A way of getting scaled mode shapes in outputonly modal testing". Proceedings of IMAC-XXI: A Conference on Structural Dynamics, February 3-6, 2003, Kissimmee, Florida.

13. Brown TG. (2000). Ice loads on the piers of the Confederation Bridge, Canada. Structural Engineer, 78(5): 18-23.

14. Brownjohn J.M.W. Xia P-Q., Hao H., Xia Y. (2001). "Civil structure condition assessment by FE model updating: methodology and case studies". Finite Elements in Analysis and Design 37:761-775.

15. CPCA -Canadian Portland Cement Association- (1995). Design and control of concrete mixtures. $6^{\text {th }}$ Edition, CPCA, Ottawa, Ontario.

16. CPWA - Canadian Public Works Association - (2003). Civil Infrastructure Systems Technology Road Map 2003-2013: A national consensus on preserving Canadian community lifelines, Toronto, Ontario, http://www.cpwa.net/Documents/TRMReport english 01.pdf, January 2005.

17. Cantieni R. (1993). Vehicle/bridge dynamic interaction for highway bridges. Proceedings of the Second European Conference on Structural Dynamics: Eurodyn'93, Vol.2, Trondheim, Norway, June 21-23 1993.

18. Chang PC and Liu SC (2003). "Recent research in non-destructive evaluation of civil infrastructures", Journal of Materials in Civil Engineering, 15(3): 298-304.

19. Cheung MS, Tadros GS, Brown T, Dilger WH, Ghali A and Lau DT. (1997). Field monitoring and research on performance of the Confederation Bridge. Canadian Journal of Civil Engineering, 24(6):951-962.

20. De Roeck G. (2005). Use of modal data in structural health monitoring. Proceedings of the SAMCO Summer Academy, 05-09 September 2005, Zell am See, Austria.

21. Desjardins, S.L. (2004). Real-Time Computer Platform for Vibration Based Structural Health Monitoring of the Confederation Bridge, M.A.Sc. Thesis, Carleton University, Ottawa.

22. Desjardins SL, Londoño NA, Lau DT, Khoo HA. (2004). Real-Time Data Processing and Graphical User Interface (GUI) for Continuous Structural Monitoring. Proceedings 
of the $18^{\text {th }}$ Australasian Conference on the Mechanics of Structures \& Materials, December 1-3 2004, Perth, Australia.

23. Desjardins S.L., Londoño N.A., Lau D.T., Khoo H. (2006). "Real-time data processing, analysis and visualization for structural monitoring of the Confederation Bridge" Joumal of Advances in Structural Engineering, 9(1):141-157.

24. Doebling S.W. and Farrar C.R. (1996). Computation of structural flexibility for bridge health monitoring using ambient modal data. Proceedings of the $11^{\text {th }}$ Engineering Mechanics Conference, Y.K. Lin T.C. Su (eds.), Vol. 2, pp. 1114-1116.

25. Doebling SW, Farrar CR, Prime MB, and Shevitz DW. (1996). Damage identification and Health Monitoring of Structural and Mechanical Systems from Changes in Their Vibration Cbaracteristics: a Literature Review. Los Alamos National Laboratory Report LA-13070MS, Los Alamos National Laboratory, New Mexico, USA.

26. Ewins DJ. (1984). Modal testing: theory and practice. Research Studies Press (John Wiley): Letchworth, England.

27. Farrar CR, Doebling SW, Cornwell PJ, Strase EG. (1997). Variability of modal parameters measured on the Alamosa Canyon Bridge. Proceedings of the $15^{\text {th }}$ International Modal Analysis Conference, February 3-6 1997, Orlando, Florida, USA, pp. 257-263.

28. FHWA - Federal Highway Administration - (1995). Recording and coding guide for the structure inventory and appraisal of the nation's bridges, Report No. FHWA-PD-96-001, US Dept. of Transportation, Washington DC.

29. Fox R. and Kapoor M. (1968). Rate of change of eigenvalues and eigenvectors. ALA A Journal, 6:2426-2429.

30. Fricker, S., Vogel T. (2005). Site Installation and Testing of Permanent Acoustic Monitoring, Proceedings of the SAMCO Summer Academy 2005, September 5-9, 2005, Zell am See, Austria

31. Friswell MI \& JE Mottershead (1995) Finite Element Model Updating in Structural Dynamics, Kluwer Academic Publishers, 286 pp

32. Friswell MI, Penny JET, Garvey SD (1996). "A combined genetic and eigensensitivity algorithm for the location of damage in structures". Identification in Engineering Systems; proceedings of the conference held at Swansea, Wales, March 1996, pp. 357-367. 
33. Friswell MI, Mottershead JE, A hmadian H (2001). "Finite element updating using experimental test data: parametrization and regularization", Philosophical Transactions of the Royal Society of London (A), 359:169-186.

34. Gao Y. and BF Spencer Jr. (2006). "Online damage diagnosis for civil infrastructure employing a flexibility-based approach", Smart Materials and Structures, 15: 9-19.

35. Ghali A, Elbadry M, Megally S. (2000). Two-year deflections of the Confederation Bridge. Canadian Journal of Civit Engineering; 27: 1139-1149.

36. Guyan R. J. (1965). Reduction of Stiffness and Mass Matrices. ALAA Journal, 3(2):380.

37. Hayden HB, Belliveau DJ, and Prinsenberg SJ. (2003). Ice drift and draft measurements from moorings at the Confederation Bridge January - March 2002. Canadian Data Report of Hydrograpby and Ocean Sciences 163, Ocean Sciences Division, Maritimes Region, Fisheries and Oceans Canada, Bedford Institute of Oceanography, Dartmouth, Nova Scotia.

38. Haritos N. and Owen J. S. (2004) "The use of vibration data for damage detection in bridges: a comparison of system identification and pattern recognition approaches", Structural Health Monitoring, 3(2): pp 141-164.

39. Huang N. E., Z. Shen, S. R. Long, M. C. Wu, H. H. Shih, Q. Zheng, N.-C. Yen, C. C. Tung, H. H. Liu (1998). The empirical mode decomposition and the Hilbert spectrum for nonlinear and non-stationary time series analysis. Proceedings of the Royal Society A: Mathematical, Pbysical and Engineering Sciences, 454(1971): 903-995 DOI:10.1098/rspa.1998.0193

40. Humar J.L. (1990). Dynamics of Structures. Prentice Hall, Englewood Cliffs, New Jersey, USA.

41. Humar J., Bagchi A., and Xu H. (2006 paper acceptance date). Performance of vibration based techniques for the identification of structural damage, Journal of Structural Control and Health Monitoring.

42. Humar JL, Bagchi A, Xu H. (2003). Challenges in vibration-based structural health monitoring. Structural Health Monitoring and Intelligent Infrastructure: Proceedings of the First International Conference on Structural Health Monitoring and Intelligent Infrastructure, 13-15 November 2003, Tokyo, Japan. Vol. 1, pp. $503-511$.

43. Jaeger GL, Mufti AA, Tadros G, Wong P. (1997). Seismic design for the Confederation Bridge. Canadian Journal of Civil Engineering; 24: 922-933. 
44. Jaishi B. and Ren W-X. (2006). "Damage detection by finite element model updating using modal flexibility residual", Journal of Sound and Vibration, 290:369-387.

45. Katsuchi H., Yamada H., Hata K., and Kusuhara S. (2003). "Full scale measurement of vibration of Akashi-Kaikyo Bridge". Proceedings of the First International Conference on Structural Health Monitoring and Intelligent Infrastructure, 13-15 November 2003, Tokyo, Japan, Vol. 2, pp. 1059-1065.

46. Kim C-Y, Kim N, Yoon J-G and Jung D-S. (2000). Monitoring system and ambient vibration test of Namhae Suspension Bridge. Proceedings of SPIE - The International Society for Optical Engineering, Nondestructive Evaluation of Highways, Utilities, and Pipelines IV; 3995: 324-332.

47. King P, Davenport A. (1997). The treatment of wind effects for the Northumberland Strait Crossing. Proceedings of the 1996 Canada-Taiwan Workshop on Medium and Long-Span Bridges, Ottawa, Ontario, Canada; 55-73.

48. Ko JM, Sun ZG, Ni YQ. (2002). Multi-stage identification scheme for detecting damage in cable-stayed Kap Shui Mun Bridge. Engineering Structures; 24:857-868.

49. Ko JM, Chak KK, Wang JY, Ni YQ, Chan THT. (2003). Formulation of an uncertainty model relating modal parameters and environmental factors by using long-term monitoring data. Smart Structures and Materials 2003: Smart Systems and Nondestructive Evaluation for Civil Infrastructures, 3-6 March 2003, San Diego, California, in: Proceedings of SPIE Vol. 5057, pp. 298-307

50. Koh, H.M., Choo, J.F., Kim, S.K. and Kim C.Y. (2003). "Recent application and development of structural health monitoring systems and intelligent structures in Korea", Proceedings of the First International Conference on Structural Health Monitoring and Intelligent Infrastructure, 13-15 November 2003, Tokyo, Japan, Vol. 1, pp. 99-111.

51. Langohr PH, Ghali A. (1997). Dynamic Effects of Ice Forces on the Confederation Bridge. Canadian Journal of Civil Engineering, 24: 915-921.

52. Lau DT, Brown T, Cheung MS, Li WC. (2004). Dynamic modeling and behavior of the Confederation Bridge. Canadian Journal of Civil Engineering, 31:379-390.

53. Lau DT, Londoño NA, Desjardins SL. (2004). Challenges of using continuous monitoring data for structural condition assessment. Fourth International Workshop on Structural Control, 10-11 June 2004, IASC, Columbia University, New York. 
54. Lee GC, Shih TS, Chang KC. (1988a). Mechanical properties of concrete at low temperature. Journal of Cold Regions Engineering 1988; 2(1):13-24.

55. Lee GC, Shih TS, Chang KC. (1988b). Mechanical properties of high-strength concrete at low temperature. Journal of Cold Regions Engineering; 2(4):169-178.

56. Lewis, J. R., Mercer, A. D. (1984). Corrosion and marine growth on offshore structures. Papers and discussion from a meeting held at the Chemistry Department of the University of Aberdeen in September 1982. Published by: Horwood; Chicester, England.

57. Li D, Maes MA, Dilger WH. (2003). Design temperature profiles for bridge decks: experimental data and extreme value analysis. Proceedings of the annual conference of the Canadian Society for Civil Engineering, June 4-7 2003, Moncton, Nouveau-Brunswick, Canada.

58. Londoño NA, Lau DT. (2003). Variability of dynamic properties from Confederation Bridge monitoring data. Proceedings of the First International Conference on Structural Health Monitoring and Intelligent Infrastructure, 13-15 November 2003, Tokyo, Japan, Vol. 1, pp. 543-550.

59. Londoño NA, Desjardins SL, Lau DT. (2004). Use of stochastic subspace identification methods for post-disaster condition assessment of highway bridges. Proceedings of the 13th World Conference on Earthquake Engineering, August 1-6, 2004, Vancouver, Canada.

60. MacGregor JG, Kennedy DJL, Bartlett FM, Chernenko D, Maes MA, Dunaszegi L. (1997) Design criteria and load and resistance factors for the Confederation Bridge. Canadian Journal of Civil Engineering; 24: 882-897.

61. Maeck J. and G. de Roeck. (2003). Description of Z24 benchmark. Mechanical Systems and Signal Processing, 17(1):127-131; doi:10.1006/mssp.2002.1548.

62. Maia NM, Montalvão e Silva JM (eds.) (1997). Theoretical and experimental modal analysis; Research Studies Press: Taunton, Somerset, England, p. 350.

63. Mehta, P.K. (1986). Concrete: Structure, Properties and Materials, Prentice Hall.

64. Montreuil, M.A., Lau, D.T. and Brown, T.G. (1998). "A distributed data acquisition system for monitoring the Confederation Bridge", Proceedings of the 44th International Instrumentation Symposium, Reno, Nevada, May 3-7 1998, pp 318-333.

65. National Climate Archive. Environment Canada. http://www.climate.weatheroffice.ec.gc.ca, March 2005. 
66. Naumoski N, Cheung MS and Foo, S. (2002). Dynamic characteristics of the Confederation Bridge. Canadian Journal of Civil Engineering, 29:448-458.

67. Naumoski N, Cheung MS and Foo S. (2004). Dynamic performance of the Confederation Bridge due to traffic and wind. Canadian Journal of Civil Engineering; $31: 487-498$.

68. Ndambi JM, Peeters B, Maeck J, De Visscher J, Wahab MA, Vantomme J, De Roeck G, and De Wilde WP. (2000). Comparison of techniques for modal analysis of concrete structures. Engineering Structures; 22:1159-1166.

69. Nocedal J. and Wright S.J. (1999). Numerical Optimiæation, Springer, New York, USA.

70. O’Callahan J., Avitabile P., Riemer R. (1989). “System Equivalent Reduction Expansion Process (SEREP)." Seventh International Modal Analysis Conference, Las Vegas, Nevada, February 1989.

71. Pandey A.K. and Biswas M. (1994). Damage detection in structures using changes in flexibility, Journal of Sound and Vibration, 169(1):3-17.

72. Peeters B. (2000). System identification and damage detection in civil engineering. $\mathrm{PhD}$ thesis, Department of Civil Engineering, Katholieke Universiteit Leuven, Belgium.

73. Peeters B. and De Roeck G. (1999). "Reference based stochastic subspace identification for output-only modal analysis", Mechanical Systems and Signal Processing, 13(6):855-878.

74. Peeters B, Maeck J and De Roeck G. (2001). Vibration-based damage detection in civil engineering: excitation sources and temperature effects. Smart Materials and Structures, 10:518-527.

75. Peeters B. and Ventura C.E. (2003). "Comparative study of modal analysis techniques for bridge dynamic characteristics", Mechanical Systems and Signal Processing, 17(5):965988. DOI: $10.1006 / \mathrm{mssp} .2002 .1568$

76. Peeters B, Couvreur G, Razinkov O, Kundig C, Van der Auweraer H and De Roeck G. (2003). Continuous monitoring of the Øresund Bridge: system and data analysis. Proceedings of IMAC XXI, a conference and exposition on structural dynamics, February 3-6 2003, Kissimmee, Florida, USA.

77. Rohrmann RG, Baessler M, Said S, Schmid W, Ruecker WF. (2000). Structural causes of temperature affected modal data of civil structures obtained by long term monitoring. Proceedings of IMAC XVIII (in Proceedings of SPIE, Vol. 4062), pp 1-7. 
78. www.samco.org, December 2004.

79. Scheaffer R.L and J.T. McClave (1982). Statistics for Engineers. Duxbury Press, Boston.

80. Schwarz B. and M. Richardson (2003). "Scaling mode shapes obtained from operating data". Proceedings of IMAC-XXI: A Conference on Structural Dynamics, February $3-6,2003$, Kissimmee, Florida.

81. Sohn H, Dzwonczyk M, Straser EG, Kiremidjian AS, Law KH and Meng T. (1999). An experimental study of temperature effect on modal parameters of Alamosa Canyon Bridge. Earthquake Engineering and Structural Dynamics; 28:879-987.

82. Sohn H, Farrar CR, Hemez FM., Shunk DD, Stinemates DW, and Nadler BR. (2003). A Review of Structural Health Monitoring Literature: 1996-2001. Los Alamos National Laboratory Report, LA-13976-MS, Los Alamos National Laboratory, New Mexico, USA..

83. Stubbs, N., Kim, J.-T., and Farrar, C.R. (1995). Field Verification of a Nondestructive Damage Localization and Severity Estimation Algorithm. 13th International Modal Analysis Conference, 210-218.

84. Teughels A., Maeck J. and De Roeck G. (2002). Damage assessment by FE model updating using damage functions. Computers and Structures, 80(25): 1869-1879.

85. Teughels, A. (2003). Inverse modelling of civil engineering structures based on operational modal data. $\mathrm{PhD}$. thesis. Katholieke Universiteit Leuven, Belgium.

86. Toksoy T. and Aktan A.E. (1994). Bridge condition assessment by modal flexibility. Experimental mechanics, 34(3): 271-278.

87. Wang J.Y., Ko J.M., and Ni Y.Q. (2000). Modal sensitivity analysis of Tsing Ma Bridge for structural damage detection. In: Nondestructive Evaluation of Highways, Utilities and Pipelines IV, Aktan E. and Gosselin S.R. (eds.). Proceedings of SPIE Vol. 3995, pp 300-311.

88. Wenzel H. (2003). E-MOI - European Monitoring Initiative. Structural Health Monitoring and Intelligent Infrastructure: Proceedings of the First International Conference on Siructural Health Monitoring and Intelligent Infrastructure, 13-15 November 2003, Tokyo, Japan, Vol. 1, pp. $145-152$.

89. Wong KY, Lau CK and Flint AR. (2000). Planning and implementation of the structural health monitoring system for cable-supported bridges in Hong-Kong. 
Proceedings of SPIE - The International Society for Optical Engineering, Nondestructive Evaluation of Highways, Utilities and Pipelines IV; 3995:266-275.

90. Woods, H. (1968). Durability of Concrete Construction, American Concrete Institute Monograph No. 4, ACI, Detroit, Michigan.

91. Wu J.R., Li Q.S. (2006). "Structural parameter identification and damage detection for a steel structure using a two-stage finite element model updating method", Journal of Constructional Steel Research, 62:231-239.

92. Wu ZS and Abe M (eds.) (2003). Structural Health Monitoring and Intelligent Infrastructure: Proceedings of the First International Conference on Structural Health Monitoring and Intelligent Infrastructure, 13-15 November 2003, Tokyo, Japan.

93. Yanev B. (2003). Structural health monitoring as a bridge management tool. Proceedings of the First International Conference on Structural Health Monitoring and Intelligent Infrastructure, 13-15 November 2003, Tokyo, Japan.

94. Yang JN, Lei Y, Pan S, Huang N. (2003). System identification of linear structures based on Hilbert-Huang spectral analysis. Part 2: complex modes. Earthquake Engineering and Structural Dynamics; 32:1533-1554.

95. Yang J. N., Y. Lei, S. Lin, and N. Huang. (2004). Hilbert-Huang Based Approach for Structural Damage Detection. J. Engrg. Mech., 30(1): 85-95

96. Zhang M. (2001). System identification analysis of the dynamic monitoring data of the Confederation Bridge, MASc Thesis, Department of Civil and Environmental Engineering, Carleton University, Ottawa, Canada. 